\title{
NEUTRON SCATTERING STUDIES OF WATER DIFFUSION NEAR THE INTERFACE OF MODEL CELL MEMBRANES
}

\author{
A Thesis presented to \\ the Faculty of the Graduate School \\ at the University of Missouri
}

In Partial Fulfillment

of the Requirements for the Degree

Doctor of Philosophy

\section{by \\ ZACHARY BUCK}

Dr. Haskell Taub, Thesis Supervisor

May 2018 
The undersigned, appointed by the Dean of the Graduate School, have examined the dissertation entitled:

\section{NEUTRON SCATTERING STUDIES OF WATER DIFFUSION NEAR THE INTERFACE OF MODEL CELL MEMBRANES}

presented by Zachary Buck, a candidate for the degree of Doctor of Philosophy and hereby certify that, in their opinion, it is worthy of acceptance.

Dr. Haskell Taub

Dr. Helmut Kaiser

Dr. Gavin King

Dr. Ioan Kosztin

Dr. Peter Cornish 


\section{ACKNOWLEDGMENTS}

I would like to express my gratitude to my academic advisor, Dr. Haskell Taub, for his advice, guidance, and outstanding patience during my time as a graduate student at the University of Missouri - Columbia. I also wish to thank my reactor supervisor, Dr. Helmut Kaiser, for his essential role in advising me on neutron scattering instrumentation data interpretation at the University of Missouri Research Reactor (MURR). I am very thankful to my predecessor, Andrew Miskowiec, for his help and the transfer of knowledge regarding fabrication of single-supported lipid bilayer membranes and analysis of QENS spectra.

I am very fortunate to have had an opportunity to work with Dr. Eugene Mamontov at the Spallation Neutron Source at Oak Ridge National Laboratory during my participation in the Office of Science Graduate Student Researcher (SCGSR) program, and I thank him for his mentorship. I also want to thank Dr. Madhusudan Tyagi at NIST Center for Neutron Research for his assistance during experiments and data analysis on the High Flux Backscattering Spectrometer (HFBS).

I appreciate the many conversations I have had with students and postdocs of Dr. Gavin King's lab regarding atomic force microscopy (AFM) and our related research projects and goals.

Lastly, my education in neutron scattering and hands-on experience working at national laboratories would not have been possible without the support of the NSF funded Integrative Graduate Education and Research Traineeship (IGERT) program. I am incredibly grateful to the IGERT program and all its members for helping to facilitate a rewarding interdisciplinary research environment. 


\section{TABLE OF CONTENTS}

ACKNOWLEDGMENTS ................. ii

LIST OF TABLES $\ldots \ldots \ldots \ldots \ldots \ldots$ vii

LIST OF FIGURES $\ldots \ldots \ldots \ldots \ldots \ldots$ viii

ABSTRACT .................... xii

1 Introduction to Model Membranes . . . . . . . . . . . . . 1

1.1 Fabrication of Model Membrane Samples for Neutron Scattering Ex-

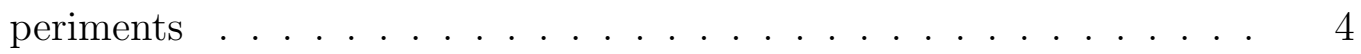

1.1.1 Zwitterionic Single-Supported DMPC Bilayers . . . . . . . 6

1.1.2 Anionic Single-Supported DMPG Bilayers . . . . . . . . 7

1.1.3 Melittin-Treated DMPC Bilayers . . . . . . . . . 9

2 Neutron Scattering from Supported DMPG Lipid Bilayers _... 11

2.1 Introduction to Backscattering Spectrometers . . . . . . . . . . 12

2.2 Elastic Neutron Scattering from Supported

DMPG Membranes . . . . . . . . . . . . . . . . . . 13

2.2.1 Comparing Elastic Scans of DMPC and DMPG . . . . . 15

2.2.2 Effects of Hydration on the Freezing and Melting of

Water in DMPG Membranes . . . . . . . . . . . . . 20

2.3 Quasielastic Neutron Scattering from DMPG Membranes ... . . . 25

2.3.1 Analysis of the QENS spectra . . . . . . . . . . 26

2.3.2 Modeling Water Diffusion . . . . . . . . . . . . . . 31 
2.4 Water Dynamics Associated with DMPG . . . . . . . . . 37

2.4.1 Comparing characteristic water types in DMPC and DMPG membranes . . . . . . . . . . . . . . . . 38

2.4.2 Search for bound water in DMPG . . . . . . . . . . . 45

2.4.3 Comparison with Molecular Dynamic Simulations . . . . . . 46

3 Neutron Diffraction from Water Hydrating Single-Supported Zwitterionic and Anionic Lipid Bilayers . . . . . . . . . 50

3.1 Introduction to Neutron Diffraction . . . . . . . . . . . 51

3.2 Experimental Setup and Operation $\ldots \ldots \ldots \ldots \ldots$

3.2.1 Instrumentation: Position Sensitive Detector (PSD)

Diffractometer . . . . . . . . . . . . . . 55

3.2 .2 Sample Environment and Control . . . . . . . . . . . 58

3.3 Results of Neutron Diffraction Measurements . . . . . . . . . . 63

3.3.1 Data Analysis and Interpretation . . . . . . . . . . 65

3.3.2 Water Structure Associated with Supported Anionic DMPG Membranes . . . . . . . . . . . . . . . 68

3.3.3 Water Structure Associated with Supported Zwitterionic DMPC Membranes . . . . . . . . . . . 76

4 Interaction of Water with DMPC Lipid Bilayer Membranes Treated with Melittin Proteins . . . . . . . . . . . 79

4.1 Introduction to Antimicrobial Peptides . . . . . . . . . 80

4.2 Properties and Insertion Mechanisms of Melittin Peptides into Lipid Bilayers . . . . . . . . . . . . . 82

4.3 Elastic Neutron Scattering from Melittin-Treated DMPC Bilayers . . . . . . . . . . . . . . . . . 85 
4.3.1 Freezing/Melting Behavior of Water Interacting with MelittinTreated DMPC Membranes . . . . . . . . . . . . 86

4.3.2 Annealing of Melittin-Treated DMPC Bilayers . . . . . . . . 90

4.4 Inelastic Neutron Scattering from

Melittin-Treated DMPC Membranes . . . . . . . . . . . 98

4.5 Quasielastic Neutron Scattering from Melittin-Treated DMPC Bilayers 103

4.5.1 Comparison of Quasielastic and Elastic Intensities from Melittin-

Treated DMPC Membranes . . . . . . . . . . . . . . 103

4.5.2 Diffusion of Water Hydrating Melittin-Treated DMPC Membranes . . . . . . . . . . . . . . . . 105

4.6 Conclusion . . . . . . . . . . . . . . . . . . . . . . . . . . . . 108

5 Microscopy of Melittin-Treated DMPC Lipid Bilayers . . . . . 110

5.1 Overview of Atomic Force Microscopy . . . . . . . . . . . . . . . 111

5.2 Effects of Temperature on Melittin-Treated

DMPC Bilayers . . . . . . . . . . . . . . . . . . . . . . 112

5.2.1 Sample Fabrication and AFM Operation . . . . . . . . . 113

5.2.2 Bilayer Thickness of Melittin-Treated DMPC

Membranes as a Function of Temperature . . . . . . . . . 117

5.2.3 Time-Dependent Annealing Effects of Melittin-Treated DMPC Bilayers . . . . . . . . . . . . . . . 126

5.3 In situ AFM Measurements on Melittin-Treated DMPC Bilayers . . . 129

5.3.1 Sample Preparation for In situ Study . . . . . . . . . . . . . 129

5.3.2 Melittin-Induced Dimple Formation in DMPC Bilayers . . . . 131

5.4 Methods of Confirming Bilayer Coverage . . . . . . . . . . . 136

5.4 .1 Force Approach Curves . . . . . . . . . . . . . . . . . . . 136 
5.4 .2 Raman Spectroscopy . . . . . . . . . . . . . . . . . . 141

A Schematic of Sample Cell used in

Neutron Scattering Measurements . . . . . . . . . . . 143

B List of Melittin-Treated DMPC Samples Measured on HFBS and

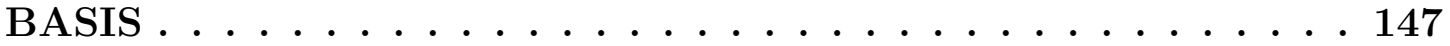

C Estimating Hydration Level from HFBS Elastic Scans . . . . . . 152

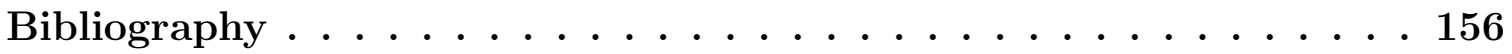

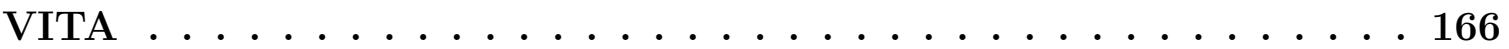




\section{LIST OF TABLES}

Table $\quad$ Page

4.1 Summary of Annealing Scans for $0.5 \mu \mathrm{M}$ melittin-treated DMPC . . . 96

B.1 List of samples measured on HFBS . . . . . . . . . . . . . . 150

B.2 List of samples measured on BASIS . . . . . . . . . . . . 150 


\section{LIST OF FIGURES}

$\begin{array}{ll}\text { Figure } & \text { Page }\end{array}$

1.1 Molecular structure of DMPC and DMPG Lipids . . . . . . . . . . 3

2.1 Backscattering minimization of neutron wavelength . . . . . . . 13

2.2 Sketch of SSLBs and location of water types . . . . . . . . . . 16

2.3 Elastic neutron scans of DMPC and DMPG membranes . . . . . . . 18

2.4 Elastic neutron scans of DMPG as a function of temperature and hydration . . . . . . . . . . . . . . . . . . . . 21

2.5 Defined regions of various water types in DMPG upon cooling . . . . 23

2.6 Quasielastic neutron scattering spectrum of wet DMPG . . . . . . . 27

2.7 Intensities from QENS spectral components of wet and dry DMPG . 29

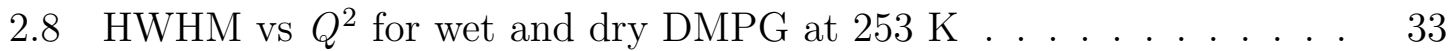

2.9 Jump-diffusion model fits to the quasielastic term in wet DMPG . . . 35

2.10 Residence times of water associated with DMPG membranes . . . . . 36

2.11 Temperature-dependent water diffusion in DMPG upon cooling . . . 40

2.12 Temperature-dependent water diffusion in DMPG upon heating . . . 43

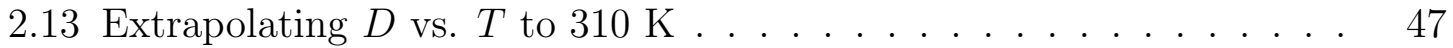

3.1 Scattering Triangle Diagram . . . . . . . . . . . . . 53 


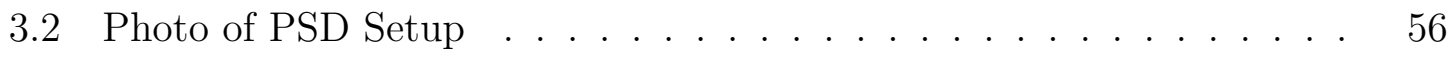

3.3 Photo of Neutron Beam at PSD Sample Position _ . . . . . . . 57

3.4 Neutron diffraction sample and cryostat on PSD . . . . . . . . . 59

3.5 Thermometry stability during DMPC diffraction experiment. . . . . 62

3.6 Theoretical Neutron Diffraction Pattern of Hexagonal $\mathrm{D}_{2} \mathrm{O} \ldots \ldots$

3.7 Gaussian Fit of hexagonal $\mathrm{D}_{2} \mathrm{O}(100)$ in $\mathrm{DMPG} \ldots \ldots \ldots \ldots$

3.8 Diffraction pattern of hexagonal $\mathrm{D}_{2} \mathrm{O}$ hydrating DMPG membranes . 69

3.9 Waterfall diffraction pattern of hexagonal $\mathrm{D}_{2} \mathrm{O}$ hydrating DMPG . . 70

3.10 Comparing HFBS elastic scans to diffraction results of DMPG . . . 71

3.11 Temperature-dependent peak positions of hexagonal $\mathrm{D}_{2} \mathrm{O}$ ice measured in DMPG upon cooling . . . . . . . . . . . . . . . . . . 74

3.12 Temperature-dependent peak positions of hexagonal $\mathrm{D}_{2} \mathrm{O}$ ice measured in DMPG upon heating . . . . . . . . . . . 75

3.13 Diffraction pattern of hexagonal $\mathrm{D}_{2} \mathrm{O}$ hydrating DMPC membranes . 77

3.14 Comparing HFBS elastic scans to diffraction results of DMPG . . . 78

4.1 Commonly cited AMP insertion mechanisms . . . . . . . . . . 81

4.2 Folded melittin peptide with colored residues . . . . . . . . . . 84

4.3 Elastic scans of melittin-treated DMPC membranes on HFBS . . . 87

4.4 Elastic thermal cycles of $0.5 \mu \mathrm{M}$ melittin on HFBS $\ldots \ldots \ldots$

4.5 Time-dependent elastic intensity measured from melittin-treated DMPC

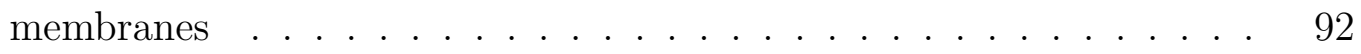

4.6 Annealing scans for 8 hours of $0.5 \mu \mathrm{M}$ melittin-treated DMPC membranes 94

4.7 Time-dependent elastic intensity measured at different temperatures from $0.5 \mu \mathrm{M}$ melittin-treated DMPC membranes . . . . . . . . 97 
4.8 Cut plots of three samples measured on SEQUOIA . . . . . . . . . 99

4.9 Density of states for water interacting with melittin-treated singlesupported DMPC membranes . . . . . . . . . . . . . . . . . . 101

4.10 Quasielastic intensity components from $0.5 \mu \mathrm{M}$ melittin-treated DMPC 104

4.11 Diffusion coefficients of water associated with $0.5 \mu \mathrm{M}$ melittin-treated

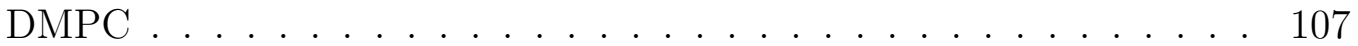

5.1 DMPC bilayer coverage as a function of lipid concentration and deposition time . . . . . . . . . . . . . . . . . . . . 114

5.2 AFM temperature calibration curve . . . . . . . . . . . . . . 116

5.3 Room temperature AFM images of partial DMPC/melittin bilayer cov-

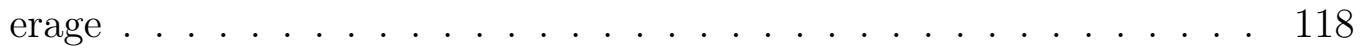

5.4 Progression of AFM images from DMPC bilayers as a function of tem-

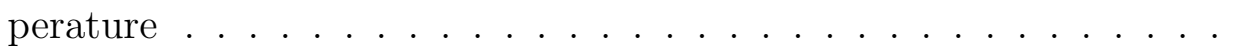

5.5 Progression of AFM images from melittin-treated DMPC bilayers as a function of temperature . . . . . . . . . . . . . . . 122

5.6 Bearing analysis of a DMPC bilayer patch at $46{ }^{\circ} \mathrm{C} \ldots \ldots . . . . .23$

5.7 Bilayer thickness of melittin-treated DMPC membranes as a function of temperature . . . . . . . . . . . . . . . 125

5.8 Evolution of AFM images of melittin-treated DMPC during annealing at $328 \mathrm{~K} \ldots \ldots \ldots \ldots \ldots \ldots$

5.9 Time-dependent Bilayer Roughness of Melittin-Treated DMPC . . . . 127

5.10 Melittin induced dimple formation in DMPC . . . . . . . . . . . 132

5.11 Melittin induced membrane destruction in DMPC . . . . . . . . . . 135

5.12 Force approach curves and roughness measurements from DMPC . . 138 
5.13 Force approach curves on DMPC multilayers . . . . . . . . . . . . . . 140

5.14 Raman spectrum of DMPC supported on $\mathrm{Si}$. . . . . . . . . . . . . . 142

A.1 Sample cell schematic 1. . . . . . . . . . . . . . . . . . . 144

A.2 Sample cell schematic 2 . . . . . . . . . . . . . . . 145

A.3 Sample cell schematic $3 \ldots \ldots$. . . . . . . . . . . . . . . 146

B.1 FWS history of $0.5 \mu \mathrm{M}$ melittin-treated DMPC . . . . . . . . 151

C.1 Elastic neutron scan of $\mathrm{C}_{32}$ calibration sample . . . . . . . . . . 153 


\begin{abstract}
Membrane proteins represent a significant frontier in structural biology they are ubiquitous in nature and perform a variety of tasks that help govern cellular activity. Their structure, insertion mechanisms, and function largely depend on the interactions between peptide-lipid domains and the hydrating water. Therefore, the dynamics of the membrane-associated water and its interaction with embedded proteins remain some of the most fundamental issues in biological physics today.

Single-supported lipid bilayers (SSLBs) provide model systems for investigating their structural and dynamical properties via atomic force microscopy (AFM) and quasielastic neutron scattering (QENS), respectively. QENS measurements on SSLBs comprised of zwitterionic (DMPC) and anionic (DMPG) lipids reveal vastly different freezing/melting behavior of their hydration water, while also elucidating various types of membrane-associated water characterized by their translational diffusion rates. Moreover, results from temperature-dependent neutron diffraction measurements on SSLBs have established a correlation between the formation of various crystalline ice structures and freezing/melting transitions observed in the elastic component of their QENS spectra, thereby confirming the various growth modes of the membrane-associated ice.

We have since enhanced the complexity and biological relevance of such systems by incorporating the antimicrobial peptide, melittin, into a DMPC membrane. On monitoring the incoherent elastic neutron intensity as a function of temperature from melittin-treated DMPC membranes, we observe an abrupt freezing transition of the associated water not seen in the bare membrane case. Moreover, the change in elastic
\end{abstract}


intensity of this freezing step increases in proportion to peptide concentration, suggesting that water could be freezing onto membrane-bound melittin. In addition to bulk-like water present in the sample, analysis of the quasielastic spectra collected provides evidence of a second water type that diffuses more slowly and freezes at a higher temperature than the bulk-like water. Furthermore, in situ AFM studies reveal the formation of dimple-like features on the surfaces of such membranes when melittin concentrations exceed $0.5 \mu \mathrm{M}$. These changes induced in the bilayer have been interpreted as aggregates of membrane-bound melittin responsible for the altered freezing behavior and dynamics of the hydration water.

An unexpected time dependence of the elastically-scattered neutron intensity was observed when membranes of DMPC treated with $0.5 \mu \mathrm{M}$ melittin were annealed in the temperature range $325 \mathrm{~K}<T<340 \mathrm{~K}$, an effect not present in DMPC membranes treated with other melittin concentrations. These results are consistent with the slowing down of hydrogen nuclei and anchoring of surface-bound melittin peptides while interacting with DMPC membranes. 


\section{Chapter 1}

\section{Introduction to Model Membranes}

In this thesis, we will apply various microscopy and neutron scattering techniques to study the structure and dynamics of water interacting with model cell membranes. In particular, we will use quasielastic neutron scattering (QENS) to identify different water types and their respective translational diffusion rates as a function of temperature and membrane composition. In addition, scanning probe microscopy is used to elucidate morphological changes of membrane structure in the presence of proteins, revealing possible insertion mechanisms aiding in the interpretation of neutron scattering results.

Membrane proteins represent a significant frontier in structural biology - they are ubiquitous in nature and perform a variety of beautifully orchestrated tasks that govern cellular activity. Their structure and function largely depend on the combined interactions between peptide domains, lipid head groups, and the surrounding hydration water. This complex web of interactions motivates the need for a deeper understanding into the fundamental mechanisms driving molecular diffusion, protein insertion, and their associated time scales. 
Over the past few decades, techniques such as QENS and nuclear magnetic resonance (NMR) have been used to study the dynamics of water on time scales ranging from $\sim 10^{-9}-10^{-13} \mathrm{~s}[1,2]$. In our studies, we explore the application of QENS to hydrated samples of single-supported lipid bilayers (SSLB) rather than multilayer stacks of thousands of membranes $[3,4]$. The relative simplicity of SSLBs aids in the interpretation of their QENS spectra and allows them to be more easily modeled in molecular dynamics simulations (MD). In addition, the stable planar geometries of SSLBs allow their surfaces to be interrogated by atomic force microscopy (AFM), which has proven to be a valuable tool for studying supported membranes $[5,6]$. A depiction of an SSLB can be seen in Figure 1.1(c).

The vast complexity and diversity of naturally occurring cell membranes necessitates their simplification into systems that are more suitable for probing individual molecular interactions such as SSLBs. We therefore begin our discussion of the sample fabrication process with the building blocks of a cell membrane, the phospholipid molecules. Previously, our group has used QENS to investigate extensively the interaction of water with single-supported bilayer membranes composed of two different phospholipid species: zwitterionic DMPC (1,2-dimyristoyl-sn-glycero-3phosphorylcholine) and anionic DMPG (1,2-dimyristoyl-sn-glycero-3-3phosphoglycerol).

Figure 1.1 illustrates the structure of DMPC and DMPG lipid molecules, which are identical in every way except for the terminal sub-unit of their head groups: a positive choline in DMPC and a neutral glycerol in DMPG. Thus, we have been able to investigate how the charge state and structure of the lipid head group affects the dynamics of its hydration water. Although the interaction of water with SSLBs of DMPC has been studied extensively $[3,4,7]$, the influence of negatively charged 

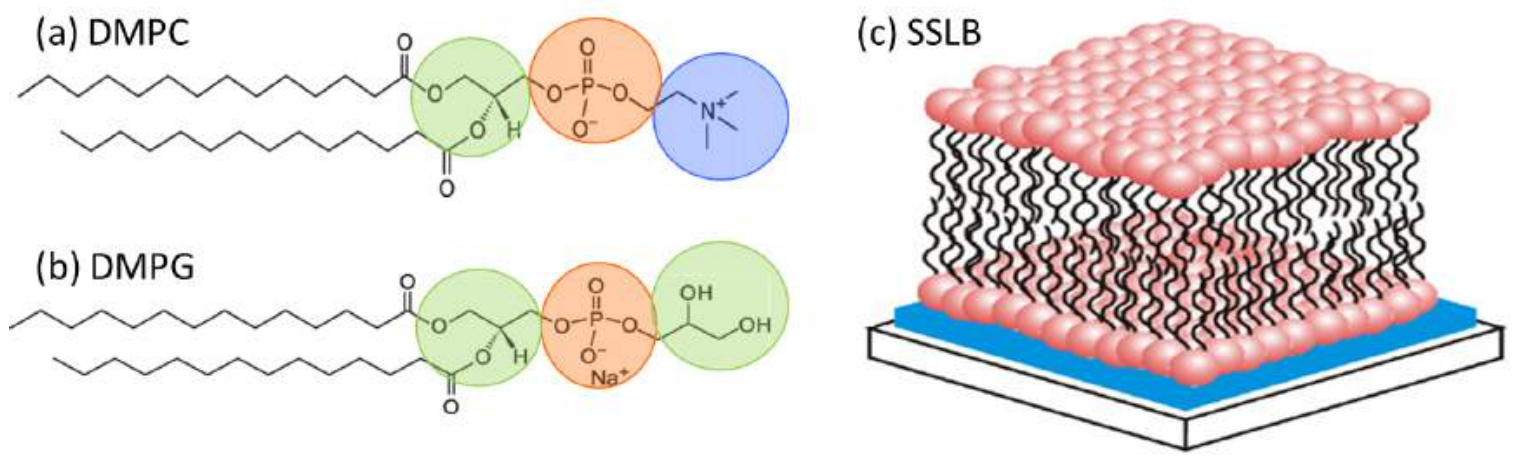

Figure 1.1: Structure of zwitterionic DMPC (a) and anionic DMPG (b) lipid molecules. The hydrophobic tail regions of both lipids are identical with each containing 14 carbon atoms. The hydrophilic head groups (depicted by colored circles) are also similar with each possessing a glycerol backbone (green) followed by a negatively charged phosphate unit (orange), but they differ in their terminal sub-units. DMPC (a) has a choline (blue) as its terminal sub-unit, rendering the molecule net neutral whereas the terminal sub-unit of DMPG is a glycerol, which results in a net negative charge of its head group. By exploiting the amphiphilic nature of these lipid molecules, it is possible to deposit a single-supported lipid bilayer depicted in (c).

DMPG on its hydration water dynamics has, until now, remained elusive. This thesis presents a series of neutron scattering results elucidating the structure (see Chapter 3) and dynamics (see Chapter 2) of the membrane-associated water in DMPG SSLBs.

In addition to a detailed analysis of surface water behavior in DMPC and DMPG SSLBs, this thesis also aims to elucidate the structure and dynamics of water hydrating more complex model membrane systems. A major contribution of this work was to incorporate proteins into SSLBs and measure their effects on the hydration water compared to the bare membrane case (no proteins). The introduction of proteins into our model membranes not only increases their complexity, but also their biological relevance by forcing them to adopt a more physiological state. The increased complexity of such systems has the potential to stimulate greater interest from the biological and medical communities, while also providing a new and interesting system 
to investigate using neutron scattering and atomic force microscopy.

\subsection{Fabrication of Model Membrane Samples for Neutron Scattering Experiments}

Understanding the fabrication process and resulting structure of DMPC and DMPG single-supported lipid bilayers is paramount as it provides a foundation for interpreting the dynamics of the membrane-associated water. Figure 1.1(c) shows a schematic representation of a typical SSLB, whose structure is similar to a bilayer sheet. Lipids are highly amphiphilic molecules, meaning they are comprised of both a hydrophilic (head group) and hydrophobic (alkyl tail) region [8]. Due to their amphiphilic nature, lipid molecules possess the ability to self-assemble in aqueous solution spontaneously to form a variety of structures including, vesicles, micelles, and bilayer sheets (see Figure 1.1(c)). There are many ways to create SSLBs; but, for purposes of this section, only one method will be discussed - vesicle fusion.

Vesicle fusion is the process in which multiple vesicles merge resulting in part from hydrophilic and hydrophobic interactions to form larger structures. They can do so while free in solution or when adsorbing onto a substrate due to the attraction of lipids to certain surfaces by electrostatic, steric, and/or van der Waals forces [9]. To facilitate vesicle fusion on a substrate, the surface properties as well as the ionic strength of the solution need to be tailored for the lipid species being used. Surfaces that are known to support lipid membranes include glass, mica, silica, and various polymers, which are often advantageous as they can minimize bilayer rigidity induced by harder surfaces. Depending on the type of measurement, we used two different 
substrates for our model membranes: mica for in situ atomic force microscopy and $\mathrm{SiO}_{2}$ for all neutron scattering samples.

$\mathrm{SiO}_{2}$-coated silicon (100) wafers $50 \mathrm{~mm}$ in diameter and $0.3 \mathrm{~mm}$ thick were purchased from WRS Materials (now Pure Wafer) [10] and used as the substrate for all samples unless specified otherwise. Wafers were cleaned using a piranha solution made by mixing sulfuric acid $\left(\mathrm{H}_{2} \mathrm{SO}_{4}\right)$ and hydrogen peroxide $\left(\mathrm{H}_{2} \mathrm{O}_{2}\right)$ typically prepared in a 3:1 ratio by volume, respectively. Piranha solution is designed to remove all organic materials from the interface, while leaving behind a homogeneous distribution of negatively charged $\mathrm{OH}^{-}$groups on the wafer surface $[11,12]$. Wafers were submerged in the piranha solution for 1 hour at $110{ }^{\circ} \mathrm{C}$ and subsequently rinsed multiple times with deionized (DI) water. The freshly cleaned wafers were then stored in a beaker of DI water where they remained until needed for lipid deposition. The deposition of both DMPC and DMPG are described in the following subsections.

Upon completion of the vesicle fusion process, wafers with deposited SSLBs were subjected to an anneal at $50{ }^{\circ} \mathrm{C}$ in air for up to 72 hours. This step was critical for neutron scattering purposes as it allowed for a more precise control over the final hydration level in the sample. Once annealed, wafers were stacked 100 high and placed in a custom aluminum sample cell before sealing with a known amount of water. For schematic diagrams of the cells, see Appendix A. Once rehydrated, the sample cell was sealed under a high-purity helium $\left({ }^{4} \mathrm{He}\right)$ atmosphere using an indium O-ring with a diameter of roughly $1 / 16$ inches. Not only is helium inert, it has a high thermal conductivity and is virtually transparent to neutrons, making it ideal as an exchange gas in temperature-dependent measurements. 


\subsubsection{Zwitterionic Single-Supported DMPC Bilayers}

DMPC $\left(\mathrm{C}_{36} \mathrm{H}_{72} \mathrm{NO}_{8} \mathrm{P}\right)$ powder was purchased from Avanti Polar lipids [Avanti] and stored at $-20^{\circ} \mathrm{C}$. The desired amount of lipid powder (typically on the order of $20 \mathrm{mg}$ ) was placed in a glass vial and dissolved in a solution of chloroform:methanol with a volume ratio of approximately $2: 1$. The chloroform/methanol and lipid solution was then dried out under a gentle stream of nitrogen until the solution was completely evaporated and only a thin film of lipid molecules remained. If necessary, the vial can be pumped for several hours to help remove any remaining moisture. The vial containing the dried lipid film was then frozen at $-20^{\circ} \mathrm{C}$ and stored until rehydration.

The lipid film was then rehydrated by suspending it in buffer solution containing $100 \mathrm{mM} \mathrm{KCl}, 5 \mathrm{mM} \mathrm{MgCl}$ and $5 \mathrm{mM}$ HEPES $\left(\mathrm{C}_{8} \mathrm{H}_{18} \mathrm{~N}_{2} \mathrm{O}_{4} \mathrm{~S}\right)$. The solution was then sonicated for several hours at $55^{\circ} \mathrm{C}$ producing multilamellar vesicles of micron size as confirmed by dynamic light scattering (DLS). The length of the sonication process can vary depending on the lipid concentration. Generally, a DMPC lipid solution will start out with a white-cloudy color before becoming more transparent after sonication. Once the solution was free of macroscopic aggregates, it was then extruded through a $200 \mathrm{~nm}$ filter and diluted to the desired concentration. Measurements by Stroumpoulis et al. have shown that vesicle fusion of DMPC onto $\mathrm{SiO}_{2}$ at lipid concentrations of $100 \mu \mathrm{g} / \mathrm{ml}$ can produce full coverage bilayers in as little as 20 minutes [13]. Therefore, our target DMPC lipid concentration was chosen to be roughly $100 \mu \mathrm{g} / \mathrm{ml}$.

Clean Si wafers (between 12 - 15) were placed into a $110 \mathrm{ml}$ solution containing DMPC vesicles with an estimated lipid concentration of $100 \mu \mathrm{g} / \mathrm{ml}$, and incubated at $45{ }^{\circ} \mathrm{C}$ for 1 hour. Once the vesicle fusion process was complete, the wafers were carefully removed from the solution and gently rinsed with DI water in an attempt 
to remove additional bilayers that may have deposited, a common feature of DMPC deposition. The wafers were then annealed, as described earlier, to remove excess moisture. DMPC surfaces were periodically imaged using AFM throughout the annealing process to confirm bilayer integrity. Once annealing was complete, the wafers were placed into an aluminum sample cell and a $120 \mu \mathrm{l}$ droplet of $\mathrm{H}_{2} \mathrm{O}$ was introduced to rehydrate the membranes fully prior to sealing the sample. In the case of neutron diffraction measurements, $\mathrm{D}_{2} \mathrm{O}$ was used to hydrate the membranes instead of $\mathrm{H}_{2} \mathrm{O}$, due to the larger coherent scattering cross-section deuterium (see Chapter 3).

\subsubsection{Anionic Single-Supported DMPG Bilayers}

We have found deposition by vesicle fusion of large, homogeneous, single-supported DMPG $\left(\mathrm{C}_{34} \mathrm{H}_{66} \mathrm{O}_{10} \mathrm{P}\right)$ membranes to be more difficult than for DMPC principally due to the sensitivity to the divalent salt concentration. However, AFM studies using a Langmuir-Blodgett technique have shown it is possible to deposit anionic membranes supported on mica [14]. Our preparation of DMPG began by suspending between 10 - $15 \mathrm{mg}$ of lipid powder (also from Avanti [Avanti]) in a chloroform solution similar to DMPC, but with the addition of a small amount of water in fluid volume ratio of 65:35:4 (chloroform:methanol:water). Just as for DMPC, the chloroform solution with dissolved DMPG was evaporated under a gentle stream of nitrogen, resulting in a thin film of lipid material uniformly deposited on the inner walls of the glass vial. The lipid film was then rehydrated with $15 \mathrm{mM} \mathrm{KCl}$ (a lower concentration than for DMPC) and $15 \mathrm{mM} \mathrm{MgCl}_{2}$ buffer solution. A higher concentration of $\mathrm{MgCl}_{2}$ than for the deposition of single membranes of DMPC was found necessary to facilitate the formation of planar membrane structures [7]. 
Upon rehydrating the DMPG solution, typically to a lipid concentration of 1 $\mathrm{mg} / \mathrm{ml}$, it was then heated to $60{ }^{\circ} \mathrm{C}$ and sonicated for several hours to break up larger aggregates. In attempts to optimize bilayer coverage, divalent salt concentrations of $\mathrm{MgCl}_{2}$ in the range $5 \mathrm{mM}-20 \mathrm{mM}$ were explored before discovering a $15 \mathrm{mM}$ concentration was optimal. Interestingly, the lower concentrations of $\mathrm{MgCl}_{2}$ did not produce any visible aggregates in the hydrated lipid solution, resulting in a clear solution even after just 5 minutes of sonication. However, higher $\mathrm{MgCl}_{2}$ concentrations resulted in large macroscopic white clusters of material that no amount of sonication could break up. It is believed the lower divalent salt concentrations weakens the attraction between lipid vesicles while in solution, thereby preventing aggregation.

Although $15 \mathrm{mM} \mathrm{MgCl}_{2}$ was found to facilitate formation of SSLBs, the DMPG solution still contained small amounts of aggregates after its rehydration and sonication. Fortunately, the solution quality was improved by extruding it through a 200 nm filter apparatus purchased from T\&T Scientific [15]. The resultant solution was clear and contained small, mostly unilamellar vesicles. However, it is possible that the extrusion process resulted in a final solution with lower lipid concentration than the previous solution containing aggregates.

After extrusion, the DMPG solution was diluted to a concentration of approximately $15 \mu \mathrm{g} / \mathrm{ml}$. Si wafers were immersed in the solution and incubated for 1 hour at $65{ }^{\circ} \mathrm{C}$ during which time vesicle fusion occurred. Upon removal, water appeared to wet the wafer, in contrast to wafers with deposited DMPC, and the remaining buffer solution was allowed to evaporate in air. Because the membrane is so weakly bound to the substrate, the salt solution cannot be rinsed away as in the DMPC preparation.

Following lipid deposition, wafers containing SSLBs of DMPG were subjected to 
the same sample sealing procedure as described for DMPC.

\subsubsection{Melittin-Treated DMPC Bilayers}

One goal in fabricating our melittin-treated DMPC bilayers was to maintain structural similarity to the bare membrane case. Therefore, we used the same methods described previously for depositing SSLBs of DMPC before exposing them to melittin peptides. In this way, the peptides were restricted to interaction only with the upper leaflet of the bilayer.

Melittin powder purchased from Sigma Aldrich [16] and Genscript [17] (purity $>96 \%$ ) was used for the AFM samples (see Chapter 5) and melittin from Sigma Aldrich (purity $>85 \%$ ) was used for the QENS samples. The powder was dissolved in an aqueous solution to form $600 \mu \mathrm{M}$ aliquots, which were stored at $-20{ }^{\circ} \mathrm{C}$ until further use. Upon thawing, the melittin aliquots were diluted to obtain solutions with peptide concentrations ranging from $0.1 \mu \mathrm{M}$ to $1.0 \mu \mathrm{M}$ in a buffer containing $10 \mathrm{mM}$ HEPES and $150 \mathrm{mM} \mathrm{NaCl} \mathrm{pH}$ 7.2. These salt concentrations have been found to be optimal in facilitating the deposition of homogeneous melittin-treated single-supported bilayers [18]. In addition to HEPES, other common buffer solutions containing Tris and phosphate have also been used to treat DMPC bilayers with melittin. No significant changes were observed in the peptide reaction enthalpy with the membrane, suggesting all three buffers result in similar lipid binding affinities for melittin [19]. Furthermore, peptide concentrations near $1.0 \mu \mathrm{M}$ were found to induce morphological changes in the DMPC membrane without causing its rupture and, therefore, were desirable for neutron scattering experiments. However, melittin concentrations exceeding $1.0 \mu \mathrm{M}$ were also investigated during in-situ AFM studies, 
which did result in destruction of the bilayer.

Once a desired melittin concentration was established, previously prepared singlesupported DMPC bilayers were submerged in the peptide solution and incubated at $50{ }^{\circ} \mathrm{C}$ for 4 hours. The binding affinity for melittin to DMPC membranes is greater when the lipids are in their fluid-phase compared to their more ordered gel-phase [20], motivating the elevated deposition temperature. Similarly prepared melittin-treated bilayers have been characterized in situ using a quartz-crystal microbalance [21]. The observed time scale over which such peptide-membrane interactions occurred was measured to be on order of several hours for the same temperature we have used. We periodically flipped the wafers and stirred the solutions gently during deposition in order to ensure all the available membrane surface area was exposed. After their exposure to the peptides, the wafers containing melittin-treated DMPC bilayers were carefully removed from the solution, rinsed with DI water, and placed in aluminum drying trays in which they could be annealed before being sealed in a sample can. The sealing procedure for melittin-treated DMPC bilayers was the same for bare membrane cases. 


\section{Chapter 2}

\section{Neutron Scattering from}

\section{Supported DMPG Lipid Bilayers}

In this chapter, we examine results obtained from the elastic and quasielastic incoherent scattering of neutrons from single-supported lipid bilayers (SSLB) of anionic DMPG as a function of temperature. The goal of these measurements was twofold. First, we aimed to identify various water types associated with a DMPG membrane by their freezing and melting behavior through elastic scans. Secondly, we performed quasielastic neutron scattering (QENS) to quantify lateral diffusion rates of the various water types.

Two complementary state-of-the-art backscattering spectrometers were used in this study. The High Flux Backscattering Spectrometer (HFBS) located at the NIST Center for Neutron Research (NCNR) in Gaithersburg, MD was used to gather elastic scans. Subsequent quasielastic spectra was then collected using the Backscattering Silicon Spectrometer (BASIS) at the Spallation Neutron Source at Oak Ridge National Laboratory (ORNL), TN. 


\subsection{Introduction to Backscattering Spectrometers}

Backscattering takes advantage of the fact that the spread in wavelength $\Delta \lambda$ of a Bragg-diffracted neutron is minimized when its scattering angle $2 \theta$ approaches 180 degrees. This principle is illustrated in Figure 2.1, which plots the wavelength of a neutron (Bragg's Law) as a function of Bragg angle $\theta$. Such backscattering geometries can be achieved in a variety of ways, resulting in well-defined exchanges in energy between an incident neutron and the specimen being investigated. From Bragg's Law, the spread of neutron wavelength, corresponding to the instrumental energy resolution, can be expressed as

$$
\frac{\Delta \lambda}{\lambda}=\frac{\Delta d}{d}+\frac{\Delta \theta}{\tan \theta}
$$

where, $\lambda$ is the neutron wavelength, $d$ is the lattice spacing between crystal planes, and $\theta$ is the Bragg angle. Equation 2.1 can be derived by differentiating Bragg's Law (see Equation 3.3) and dividing the result by the neutron wavelength $\lambda$. For a more complete description of Bragg's Law and its applications the reader is referred to Chapter 3, which discusses neutron diffraction from single-supported membranes hydrated with $\mathrm{D}_{2} \mathrm{O}$.

Capabilities of backscattering instrumentation are constantly increasing with stateof-the-art spectrometers able to detect energy transfers with sub- $\mu \mathrm{eV}$ resolution, corresponding to time scales of a few nanoseconds. This capability makes these instruments ideal for studying both lipid and water dynamics. Here we apply the backscattering technique to probe the dynamics of water associated with anionic lipid membranes comprised of DMPG molecules. 


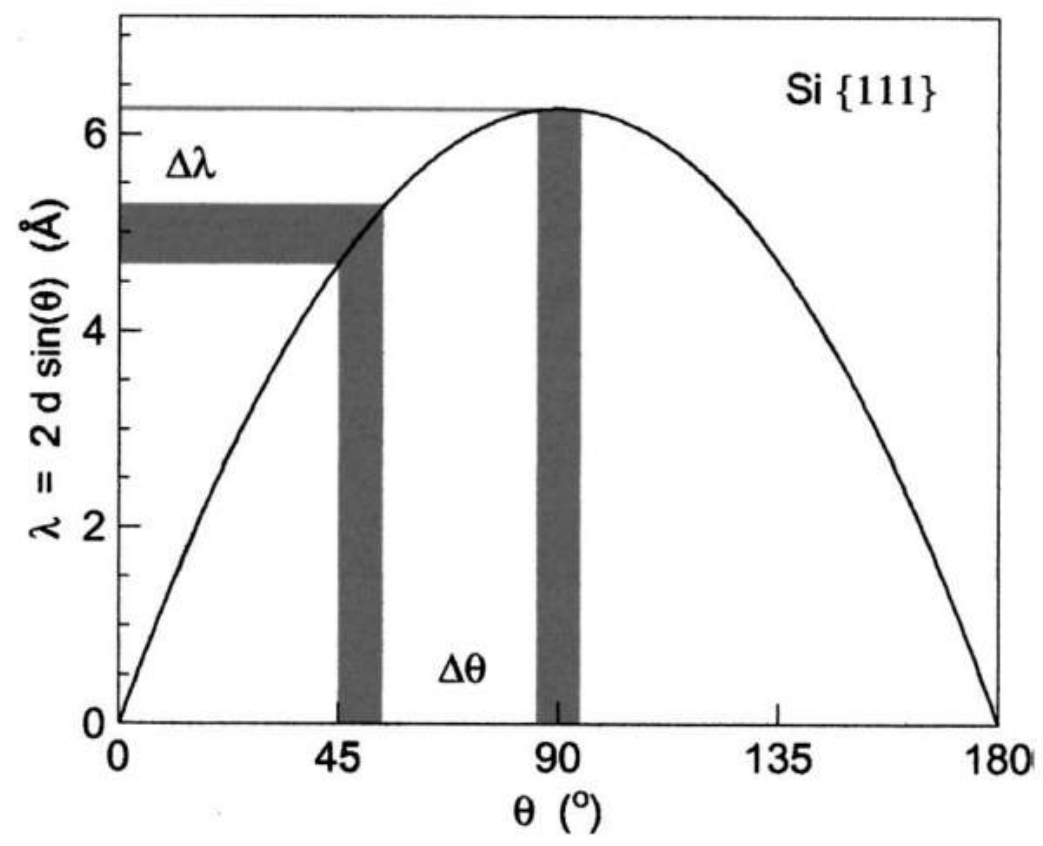

Figure 2.1: Representation of the backscattering principle applied to $\mathrm{Si}(111)$ analyzer crystals on the HFBS. Equal angular spreads $\Delta \theta$ are shown as vertical shaded bands, each corresponding to a respective spread in neutron wavelength $\Delta \lambda$ (horizontal shaded bands). Minimization of the spread in neutron wavelength occurs when $\theta$ is centered at a Bragg angle of $90^{\circ}$ (i.e., scattering angle $2 \theta=180^{\circ}$ ). This figure has been taken from Ref. [22].

\section{$2.2 \quad$ Elastic Neutron Scattering from Supported DMPG Membranes}

Here we will discuss the temperature-dependent intensity of neutrons scattered elastically and incoherently from SSLBs of DMPG hydrated with increasing amounts of water measured on the High Flux Backscattering Spectrometer (HFBS). Elastic neutron scans are a critical preliminary measurement as they provide insight into the interaction strength of water with these membranes and help identify temper- 
ature regions and phase transitions suitable for a more detailed investigation using quasielastic techniques.

The HFBS possesses an oscillating monochromator with the ability of Doppler shifting the wavelength of incident neutrons to achieve a dynamic range up to \pm $36 \mu \mathrm{eV}$ which is required to observe quasielastic behavior [22]. However, fixing the Doppler monochromator restricts the detection to only those neutrons scattered with energy transfers less than $\sim 1 \mu \mathrm{eV}$, (the energy resolution of the instrument), corresponding to a time scale of 4 ns. Neutrons that scatter from the sample experiencing energy transfers within the instrumental resolution are considered to be elastic and are the focus of this section.

The elastic signal observed for our membrane samples is dominated by the enormous 80 -barn $\left(1\right.$ barn $\left.=10^{-28} \mathrm{~m}^{2}\right)$ incoherent scattering cross-section of hydrogen. Therefore, an increase in elastic intensity is proportional to the the number of hydrogen nuclei moving on a time scale slower than 4 ns. Because most of the hydrogen originates from $\mathrm{H}_{2} \mathrm{O}$ molecules, an increase or decrease of elastic intensity is interpreted as the freezing or melting of water, respectively.

We note that using our relatively large samples, the elastic scans provide a sensitivity to water freezing transitions that is inaccessible to differential scanning calorimetry (DSC) with commercially available instruments. Scaling the water content of our 100wafer neutron scattering sample $(\sim 120 \mu \mathrm{l})$ by the surface area of wafers that would fit in the sample volume of a commercial DSC apparatus yields a water content of $\sim 0.06 \mu \mathrm{l}$ or one to two orders of magnitude less water than typically required to detect freezing transitions. 


\subsubsection{Comparing Elastic Scans of DMPC and DMPG}

Before exploring the effects of hydration on DMPG membranes, we first wish to understand how the structure and charge state of the lipid head group affects the freezing and melting behavior of its membrane-associated water. Therefore, elastic neutron scans of previously measured zwitterionic DMPC membranes [3] were compared with those of anionic DMPG.

We have found our samples of single-supported zwitterionic (DMPC) and anionic (DMPG) bilayer membranes to be simple and homogeneous enough to allow different types of water that are common to both membranes to be distinguished. These water types are presumably determined by their local environment, which is characterized by the proximity of the water molecules to the lipid head groups, the strength of the water-head group interactions and, at low temperatures, by the proximity of water molecules to bulk ice. In addition to mobile "bulk-like" water furthest from the lipid head groups, we have identified water types termed "confined 1" and "confined 2", as depicted in Fig. 2.2, in closer proximity to the membrane. Analysis of the incoherent elastic neutron intensity from SSLBs of DMPC and DMPG allows one to distinguish between different types of water based on their freezing/melting behavior.

Figure 2.3 reveals the temperature-dependent intensities of neutrons scattered incoherently and elastically from fully hydrated samples of single-supported DMPC and DMPG membranes $[3,4]$ as measured on the HFBS. Intensities have been summed over all wave vector transfers ( 16 detectors in total covering momentum transfers 0.25 $\AA^{-1}<Q<1.75 \AA^{-1}$ ) and normalized to unity at $270 \mathrm{~K}$. Normalization at $270 \mathrm{~K}$ was chosen because at this temperature the supported DMPC and DMPG bilayers are in their gel phase in which center-of-mass motion of the lipid molecule occurs 
(a)

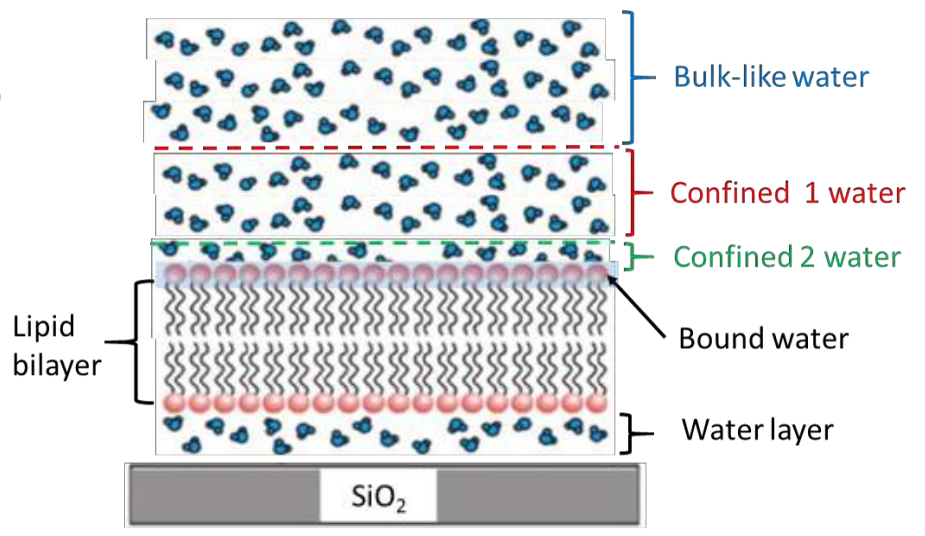

(b)

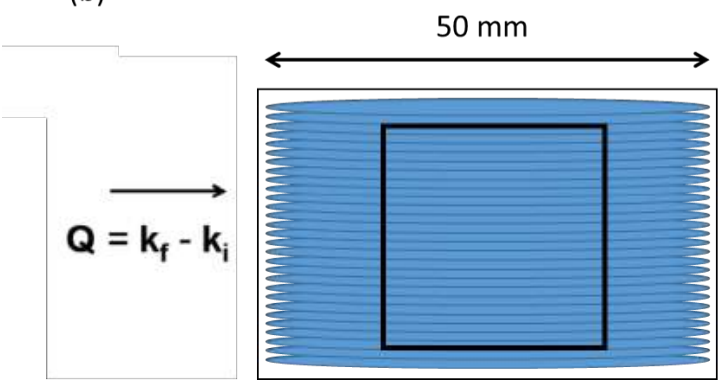

Figure 2.2: (a) Sketch of a hydrated single-supported bilayer membrane. Water types inferred for the DMPC and DMPG membranes from the freezing/melting behavior of their associated water and the analysis of their QENS spectra are indicated schematically: bulk-like, confined 1 and 2, and bound. (b) Schematic diagram of the neutron scattering sample consisting of a stack of $\mathrm{Si}(100)$ wafers, indicating the direction of the neutron wave vector transfer $Q$ with respect to the wafer plane. The black square indicates the cross sectional area of the neutron beam incident on the sample. 
on a time scale too slow to be detected by the instrument. However, there does exist a very weak component from motion of the lipid tails that contributes to the quasielastic intensity, which is only present at large momentum transfers $(Q>1.5$ $\AA^{-1}$ ). The intensity of this component was found to be roughly 30 times weaker than the dominating quasielastic term at lower temperatures (see Section 2.4.2).

Nuclei in the single-crystal silicon substrates provide the dominant contribution to the elastic intensity at temperatures above $270 \mathrm{~K}$. At these temperatures, motion of hydrogen in the liquid water is faster than the time scale of the instrument and therefore cannot be detected until its motion begins to slow as indicated by an increase in elastic signal. Assuming the gel phase area per lipid of DMPC (60 $\AA^{2} /$ lipid) [23] and DMPG (47 $\AA^{2} /$ lipid) [24], we estimate the contribution to the elastic intensity from lipids to be about $27 \%$ and $31 \%$, respectively, of the total intensity observed at $270 \mathrm{~K}$. These fractions are determined by the number of silicon atoms in the substrate, the number of lipid hydrogen, and their incoherent neutron cross-sections.

It is clear from Figure 2.3 that water hydrating a DMPC membrane (Fig. 2.3(a)) exhibits significantly different freezing and melting behavior compared to water hydrating DMPG (Fig. 2.3(b)), particularly upon heating. On heating, the DMPC membrane shows a relatively abrupt decrease in its elastic intensity close to the bulk water melting point of $273 \mathrm{~K}$ with a small pre-melting effect beginning near $267 \mathrm{~K}$. In contrast, the DMPG membrane demonstrates a more gradual decrease in intensity upon heating which begins at temperatures as low as $237 \mathrm{~K}$. Moreover, there are several new features in the heating curve in DMPG (see Fig. 2.3(b)) not previously seen for DMPC. For example, there is a downward substep near $240 \mathrm{~K}$ followed by another near $260 \mathrm{~K}$ before the intensity finally levels off at $269 \mathrm{~K}$, four degrees below 


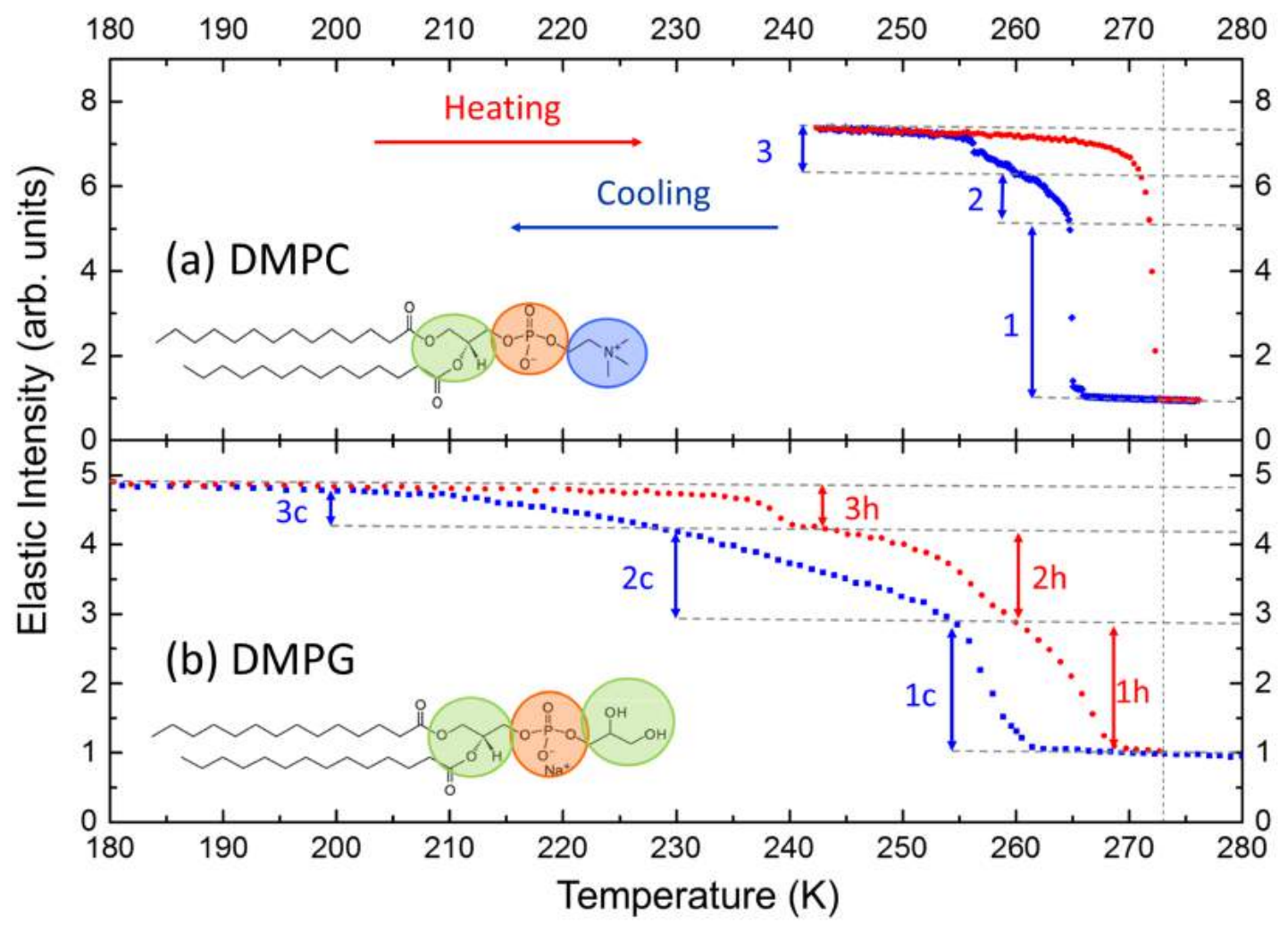

Figure 2.3: Incoherent elastic neutron intensities as a function of temperature from hydrated zwitterionic DMPC (a) and anionic DMPG (b) bilayers measured on the HFBS. Intensities were summed over all wave vector transfers and normalized to unity at $T=270 \mathrm{~K}$. Blue and red data points were taken on cooling and heating, respectively. The vertical double arrows indicate the intensity increment (decrement) associated with freezing (melting) of each water type (labeled with subscripts $1-3$ ), with horizontal dashed lines included for clarity. The vertical dashed line at $273 \mathrm{~K}$ represents the bulk melting point of water. For DMPG, the labels $\mathrm{c}$ and $\mathrm{h}$ on the arrows indicate cooling and heating, respectively; numbered substeps in its heating curve match intensity increments in its cooling curve. For DMPC, the location of the freezing transition labeled 2 was determined from the temperature dependence of the diffusion coefficient [4] (see Fig. 2.11). The insets represent the molecular structures of the two lipids, with the only difference located in the terminal subunit. 
the bulk melting point of water. These substeps seem to indicate the presence of three separate water types in the DMPG sample, each represented by vertical double red arrows in Fig. 2.3(b). Furthermore, the intensities at which these substeps occur correlate nicely with inflection points in the cooling curve of DMPG, indicating that these water types freeze and melt at different temperatures.

Upon cooling, freezing of water for the DMPG membrane occurs over a larger temperature range $(\Delta T \approx 60 \mathrm{~K})$ than for $\operatorname{DMPC}(\Delta T \approx 10 \mathrm{~K})$. Elastic intensities of DMPC have already been described previously in great detail $[3,4,7]$; however, it is worth mentioning a few important features in its cooling curve that will be relevant in later chapters (see Chapters 3 and 4). The DMPC sample displays a sharp vertical step in its elastic intensity at $265 \mathrm{~K}$ followed by a continuous increase in intensity before finally saturating at $252 \mathrm{~K}$, indicating the complete freezing of water. We interpret the initial freezing transition of water hydrating DMPC to be similar to that of bulk whereas the continuous freezing is identified with water interacting more strongly with the lipid head groups.

Rather than an abrupt freezing transition, the DMPG membrane displays a broadened upward step in elastic intensity upon cooling starting at $260 \mathrm{~K}$, followed by a broadened and nearly linear increase that extends to a temperature below $230 \mathrm{~K}$ before saturating at $200 \mathrm{~K}$. The inflection point in the DMPG cooling curve at $253 \mathrm{~K}$ (see Fig. 2.3(b)) occurs at an intensity where the second substep upon heating was observed. This inflection point has been interpreted as the complete freezing out of bulk-like water and the start of freezing of a second water type, defined as 'confined 1' water. Confined 1 water is believed to be located in a region between the membrane and the newly formed bulk-like ice. 
Although it is difficult to discern from the elastic scans alone, there is a subtle second inflection point in the DMPG cooling curve at $230 \mathrm{~K}$. Once again, this low temperature inflection point occurs at an intensity correlating with a heating substep $(\sim 240 \mathrm{~K})$ and indicates the beginning of solidification of a third type of water. We propose this third type of water is located closest to the lipid head groups, where it can interact more strongly with the membrane. Later in this chapter, we will discuss quasielastic neutron scattering measurements performed on DMPG membranes whose results provide further evidence of three types of water defined by differences in their dynamics.

\subsubsection{Effects of Hydration on the Freezing and Melting of Water in DMPG Membranes}

In Figure 2.4, we compare HFBS elastic scans of the DMPG sample in Figure 2.3(b) with three other DMPG samples hydrated with lesser amounts of water. The three samples with the most water exhibit a qualitatively similar and reproducible temperature dependence of the elastic intensity upon cooling. They differ principally in that both the onset temperature of the increase in elastic intensity and the magnitude of its initial rise, which tends to decrease with lower water content. Their similarity can be seen more clearly in Figure 2.5 where we define three characteristic temperature ranges on cooling: Region 1 ( $253 \mathrm{~K}<T<270 \mathrm{~K}$ ) corresponding to bulk-like water in which the initial rapid rise in the elastic intensity occurs; Region $2(230 \mathrm{~K}<T<$ $253 \mathrm{~K}$ ) corresponding to confined 1 water in which the intensity increases more slowly and nearly linearly in temperature; and Region $3(T<230 \mathrm{~K})$ corresponding to a third type of water over which the intensity begins to level off. The behavior of the 


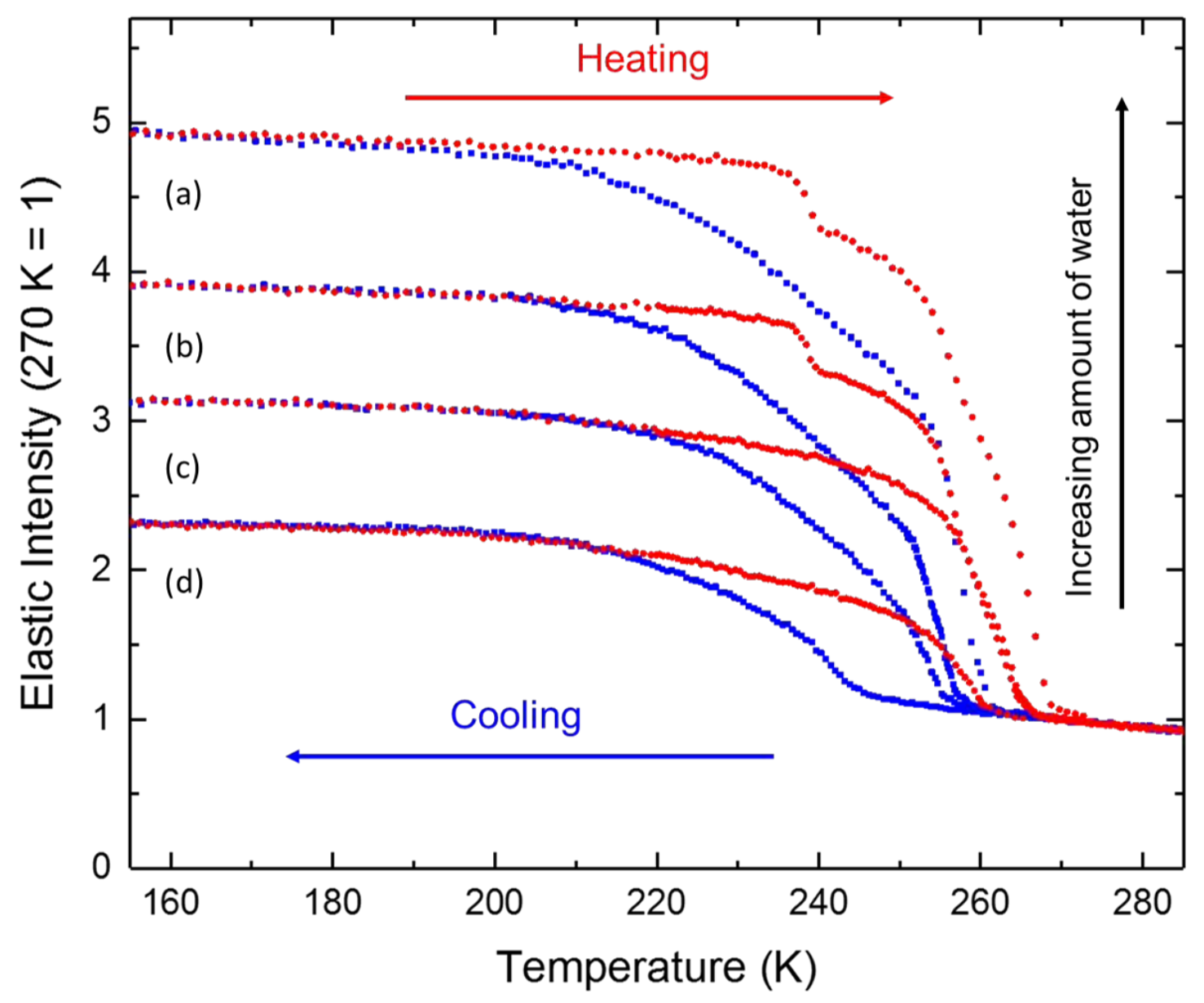

Figure 2.4: Temperature- and hydration-dependent elastic neutron scans measured form four different DMPG samples on the HFBS. Varying the amounts of water in each sample alters its freezing and melting transitions, with the greatest effects observed at high hydration levels. The corresponding equivalent water thicknesses (see text for definition) are: (a) $71 \mathrm{~nm}$, (b) $52 \mathrm{~nm}$, (c) $39 \mathrm{~nm}$, and (d) $23 \mathrm{~nm}$. The data has been summed over all wave vector transfers and normalized to unity at $T=$ $270 \mathrm{~K}$. The blue and red data points were taken on cooling $(0.04 \mathrm{~K} / \mathrm{min})$ and heating $(0.1 \mathrm{~K} / \mathrm{min})$, respectively. 
sample with the least amount of water (Figure 2.5(d)) differs from the other three in that the initial rise in elastic intensity occurs at a lower temperature of $\sim 244 \mathrm{~K}$, and the transition from a nearly linear intensity increase in Region 2 to the saturating behavior in Region 3 is not as well defined as the samples with greater hydration.

As alluded to earlier, the pairs of vertical double arrows of equal length in Fig. 2.3(b) help to identify three regions where we see reasonable agreement between the intensity decrement in each of the substeps on heating with intensity increments that occur on cooling. However, these regions are difficult to apply to the two DMPG samples having the lowest water content, particularly upon heating because they do not show any step-like decreases in intensity as seen at higher hydration. We also note that as the water content of the DMPG samples decreases, there is a corresponding decrease in the temperature at which the melting of their ice is complete. For the sample with the least amount of water (see Fig. 2.4(d)), the minimum intensity occurs at $\sim 260 \mathrm{~K}$, or about $13 \mathrm{~K}$ below the melting point of bulk ice. Moreover, ice associated with the driest DMPG sample begins to melt roughly $20 \mathrm{~K}$ below the initial temperature at which ice in the two DMPG samples having the greatest levels of hydration abruptly melts. Interestingly, although water begins to melt earlier in the two driest DMPG samples, there are no well-defined substeps in their heating curves as exhibited by the two DPMG samples with the greatest amount of hydration (see Fig. 2.4(a) and 2.4(b)).

Elastic neutron scans not only provide insight into the freezing/melting behavior of water interacting with model cell membranes, but they can also be used to quantify the amounts of water participating in such phase transitions. By measuring the temperature dependence of neutrons scattered elastically from a reference sample 


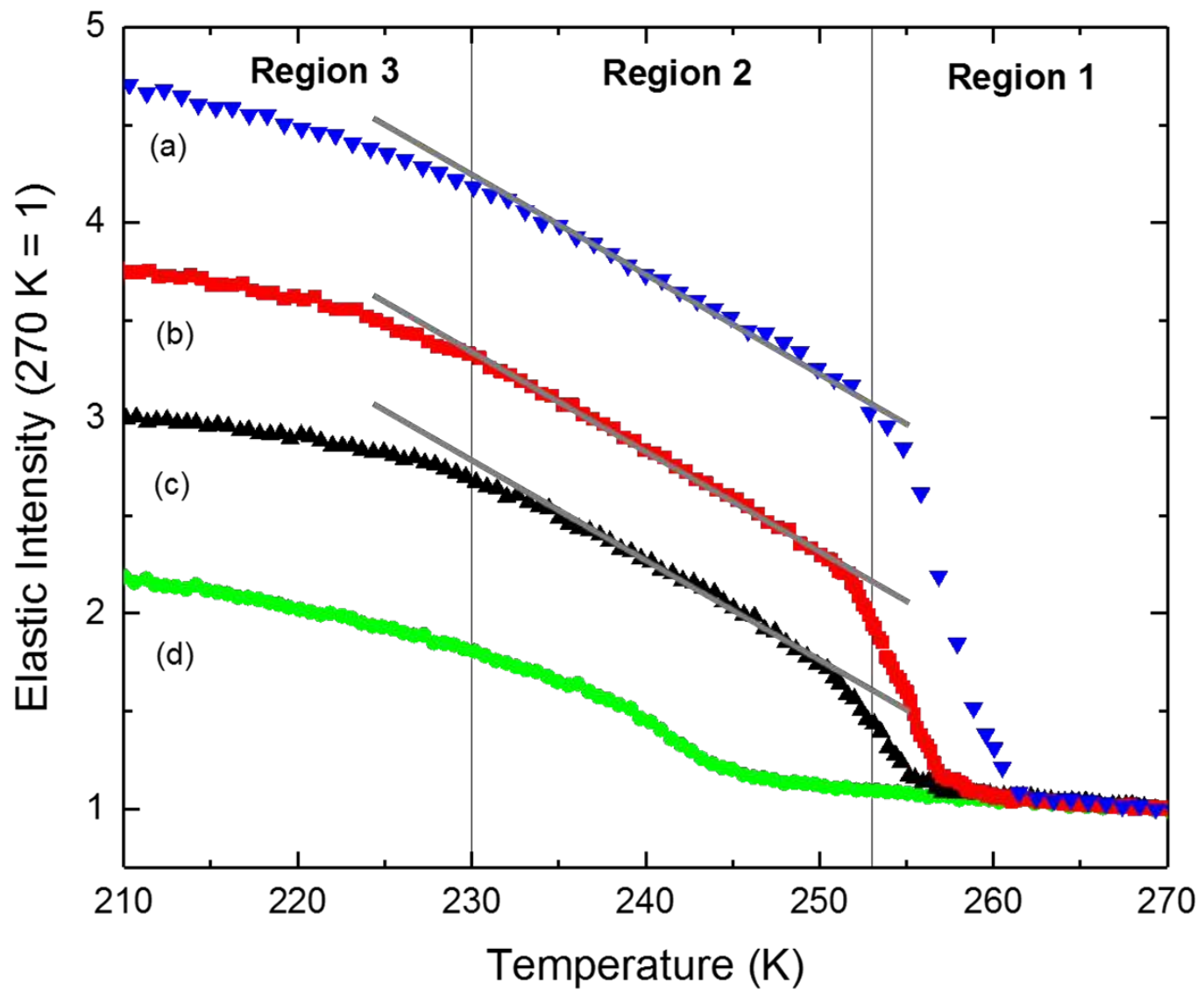

Figure 2.5: Identification of three temperature regions upon cooling four DMPG membranes. Region 1: freezing of bulk-like water above the membrane in the three samples with the highest water content $(\mathrm{a}-\mathrm{c})$ with their intensity increments scaling with the amount of water. Region 2: freezing of confined 1 water closer to the lipid head groups in the three samples with the highest water content. In Region 2, the slope of the linear intensity increase (gray lines) agrees within $4 \%$ for the three samples with the highest amount of water, indicating that the amount of confined 1 water freezing is independent of the total water content. Region 3: freezing of water closest to the membrane. The sample with the least amount of hydration (d), appears to have only a small amount of bulk-like and confined water. The four cooling curves were taken from Figure 2.4. 
of known hydrogen content, one can correlate changes in elastic intensity to the number of hydrogen nuclei. The reference sample used in estimating the number of water molecules hydrating the four DMPG samples shown in Fig. 2.4, in addition to the DMPC sample in Fig. 2.3(a), was comprised of $\mathrm{C}_{32} \mathrm{H}_{66}$ multilayers of alkane chains deposited on similar Si wafers used in the fabrication of our SSLBs. Applying this method of hydrogen quantification to the elastic scans in Fig. 2.4, we estimate equivalent water slab thickness of the four DMPG samples to be $71 \mathrm{~nm}, 52 \mathrm{~nm}, 39$ $\mathrm{nm}$, and $23 \mathrm{~nm}$, from highest to lowest hydration level. We assume the equivalent water slabs are located above each bilayer on both sides of a wafer. A description for calculating the number of hydrogen nuclei in samples of SSLBs measured on the HFBS is outlined in Appendix C.

Due to differences observed in the freezing/melting transitions of water hydrating DMPG membranes of various hydration levels, the two samples having the greatest difference in water content were chosen to be investigated further using QENS. We denote DMPG samples possessing equivalent water slab thicknesses of $71 \mathrm{~nm}$ (Fig. 2.4(a)) and $23 \mathrm{~nm}$ (Fig. 2.4(d)), as 'wet' and 'dry' DMPG, respectively. 


\subsection{Quasielastic Neutron Scattering from DMPG Membranes}

To elucidate membrane-associated water dynamics in DMPG bilayers and assist in the interpretation of their elastic scans, full quasielastic neutron scattering (QENS) spectra were collected on the backscattering spectrometer BASIS [25]. Due to their qualitatively different freezing and melting behaviors, as observed in the elastic scans, samples of dry (Fig. 2.4(d)) and wet (Fig. 2.4(a)) DMPG were investigated using QENS. The large dynamic range of BASIS $( \pm 120 \mu \mathrm{eV})$ allowed for spectra to be measured and analyzed over nearly the entire temperature range in which the elastic scans on HFBS were performed $(220<T<295 \mathrm{~K})$. Reduction of data collected on BASIS was carried out using a standard method that binned the intensities into 9 distinct momentum transfers ranging from $0.3<\AA^{-1} Q<1.9 \AA^{-1}$.

Although BASIS has a larger dynamic range, its energy resolution is slightly poorer than that on the HFBS with an elastic linewidth of $\sim 3.5 \mu \mathrm{eV}$, corresponding to a time scale of about 1 ns. Therefore, QENS measurements at the lowest temperatures,

where the slower dynamics dominate, were better suited for the HFBS due to its higher energy resolution of $\sim 1 \mu \mathrm{eV}$. 


\subsubsection{Analysis of the QENS spectra}

We fit QENS spectra using the Data Acquisition and Visualization Environment (DAVE) software [26] by folding the instrumental resolution function with a scattering law comprised of three terms: a delta function corresponding to the elastic scattering plus two Lorentzian terms representing the quasielastic scattering. Below is a simplified version of the scattering law in the absence of a background term

$$
S(\mathbf{Q}, \omega) \propto \delta(\omega)+\frac{1}{\pi} \sum_{j}^{N}\left(\frac{\Gamma_{j}}{\Gamma_{j}^{2}+\omega^{2}}\right)
$$

where $\mathrm{S}(\mathbf{Q}, \omega)$ represents the dynamic structure factor, which is a function of momentum and energy transfers, $\mathbf{Q}$ and $\omega$, respectively. The first term in Equation 2.2 is a delta function, which represents strictly elastic scattering from the sample. The summation in Equation 2.2 is over the number $N$ of all quasielastic components used to fit the spectra, which in this case are represented by Lorentzians, with half-widthhalf-maximum (HWHM) $\Gamma$. As mentioned above, we used two Lorentzian terms $(N=2)$ to fit the QENS spectra: one broad, representing faster motions and one narrow representing slower motions. The instrumental resolution function is unique for each sample, and is acquired by cooling to very low temperatures $(T<5 \mathrm{~K})$, at which point nearly all nuclei motion has ceased. The elastic and quasielastic intensity contributions are then convoluted with the instrumental resolution function and summed to fit the spectra.

The decomposition of a BASIS spectrum into these three components and a linear background term is illustrated in Figure 2.6 for the wet DMPG sample at a temperature of $253 \mathrm{~K}$ and $Q=0.9 \AA^{-1}$. Spectra from wet and dry DMPG samples having 


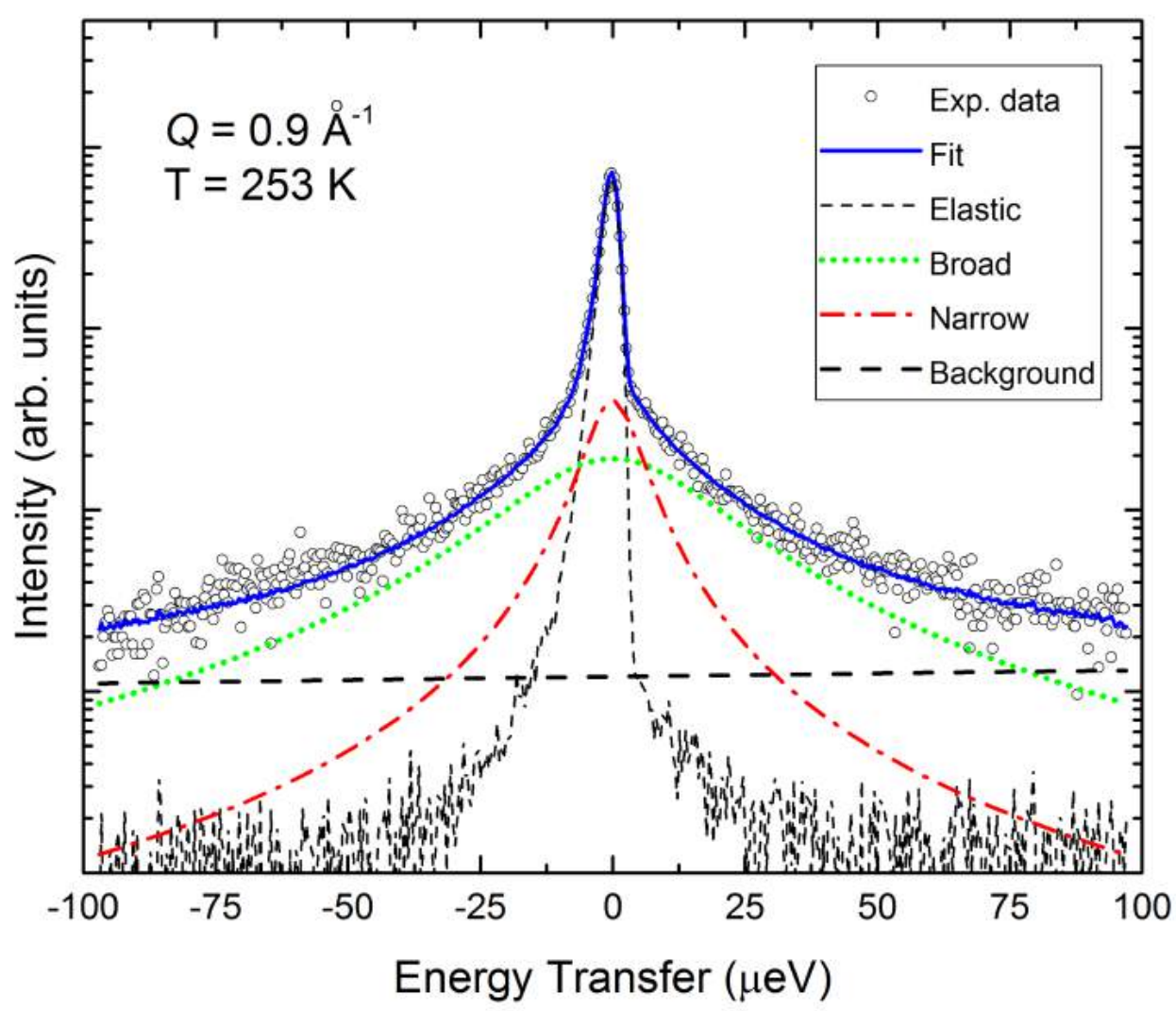

Figure 2.6: QENS spectrum of the wet DMPG sample measured on BASIS at $Q=$ $0.9 \AA^{-1}$ and $T=253 \mathrm{~K}$ upon cooling. The data points (open circles) have been fitted by using a scattering law similar to Equation 2.2. A linear background term (bold dashed line) has been included with the other three components, which have been folded with the instrumental resolution function: the delta function (dashed black curve), a broad Lorentzian (green dotted curve) representing faster motion, and a narrow Lorentzian (red dot-dash curve) representing slower motion. The best fit to the spectrum (blue solid curve) is a result of summing all spectral components. 
effective water slab thicknesses of $71 \mathrm{~nm}$ and $23 \mathrm{~nm}$, respectively, were collected upon cooling and heating and analyzed by applying the fitting methods described here. Elastic intensities obtained on BASIS from the DMPG samples were compared with previously measured elastic scans on HFBS. Parameters of HWHM were extracted from the quasielastic terms and yielded diffusion coefficients associated with various water types as will be discussed later in this chapter. It should be noted that not all temperatures and $Q$ values required two Lorentzian terms to fit their spectra, particularly those measured at lower temperatures.

At high temperatures, there exist greater quantities of mobile water with each type possessing a rate of diffusion as described by their characteristic HWHM as a function of $Q$ (see Section 2.3.2). It was found that two Lorentzians were required to fit the high-temperature QENS spectra collected from both wet and dry samples of DMPG. However, at lower temperatures, the dynamics associated with the broad component are slowed down as indicated by a decrease in HWHM. As the widths of the broad component decrease, they become comparable to those associated with the narrow component, which can lead to a mixing of the two terms and an exchange of their intensities. Therefore, to avoid any competition between parameters of the quasielastic components, a one-Lorentzian fit to the spectra was enforced beginning at temperatures at which the mixing of terms was first observed.

Figure 2.7 shows the temperature-dependent intensities from elastic and quasielastic terms used to fit the QENS spectra of wet and dry samples of DMPG. To check the compatibility between the two instruments, we first compared the elastic intensities obtained on the HFBS with those collected on BASIS (i.e., the integrated intensity of the delta-function component folded with the resolution function shown by the 

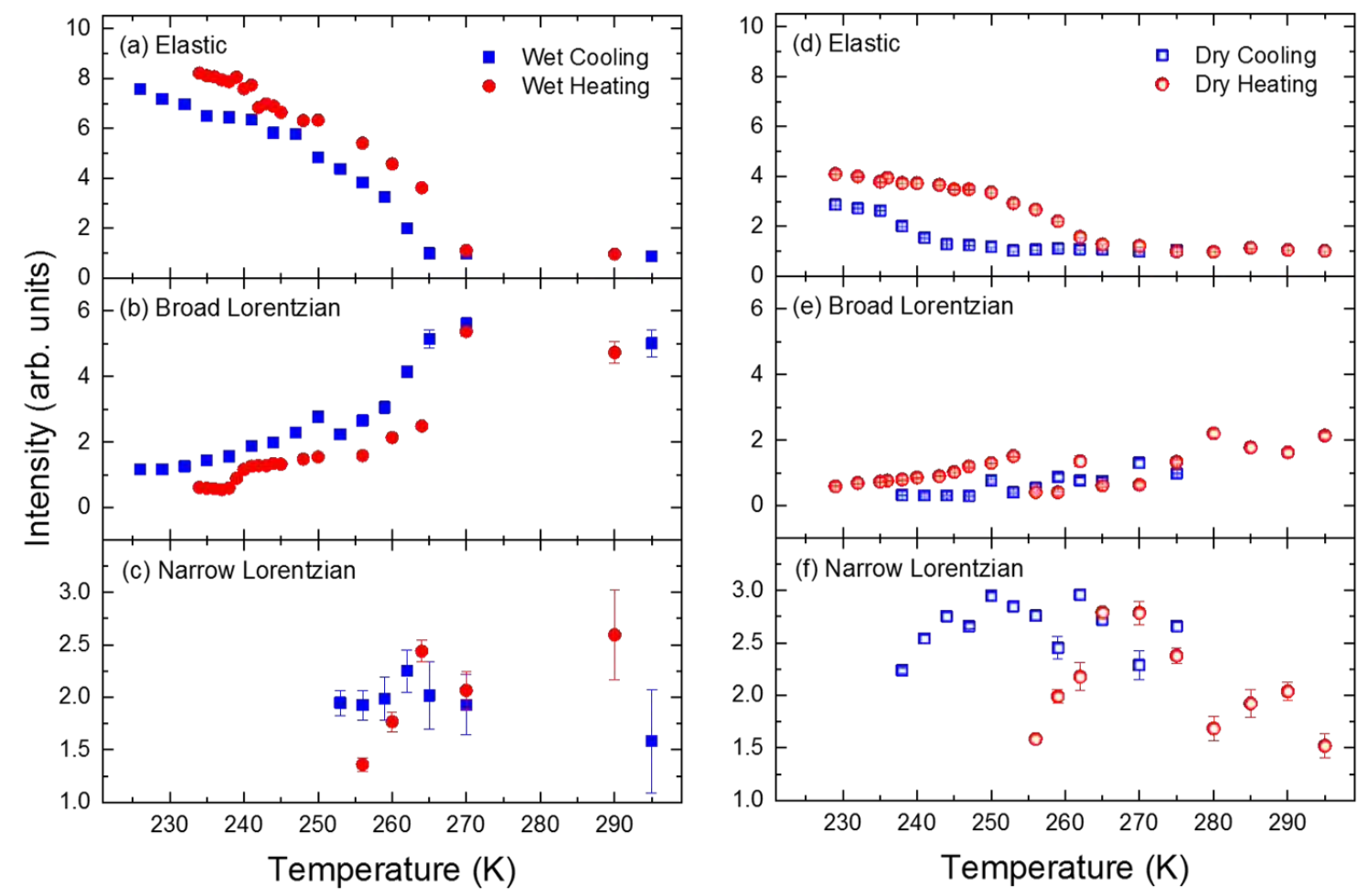

Figure 2.7: Delta function and Lorentzian component intensities from QENS spectra measured on BASIS for wet (left) and dry (right) DMPG samples as a function of temperature. Cooling and heating data for the wet and dry samples are shown with blue squares and red circles, respectively. The elastic intensities for wet (a) the dry (d) DMPG samples were averaged over all $Q$ and normalized to unity at $T=270 \mathrm{~K}$. Intensities of the two Lorentzian components were averaged over wave vector transfers $0.5 \AA^{-1}, 0.7 \AA^{-1}$, and $0.9 \AA^{-1}$, which were used in the determination of the diffusion coefficient and have been normalized to allow comparison with their respective elastic intensities. 
dashed black curve in Fig. 2.6). Elastic intensities upon cooling (blue points in Fig. 2.7) from both instruments agree reasonably well as can be seen by comparing the results from Figures 2.4(a) and (d) with 2.7(a) and (d) for wet and dry samples, respectively.

Over a temperature range of $230 \mathrm{~K}<T<270 \mathrm{~K}$ in the BASIS spectra, we find an overall change in the delta-function intensity of the dry DMPG sample on cooling (see Fig. 2.7(d)) is about a factor of 3.6 less than for the wet sample. Similarly, from HFBS measurements (see Fig. 2.4(a) and (d)), the increase in the elastic intensity of the dry sample over the same temperature range is about a factor of 3 less than for the wet sample, which provides confidence in the reproducibility and therefore the sample quality; however, there do exist some slight discrepancies. For example, the onset temperature of the steep increase in elastic intensity on cooling (HFBS) is about three degrees lower than for the delta-function intensity (BASIS).

Although we are unable to identify precisely the origin of these discrepancies, we consider several possible explanations. Perhaps the most obvious is the fact that the two spectrometers differ in their energy resolution. Additionally, the nature of the measurements are slightly different in that data collected on the HFBS was done so by continuously ramping the temperature whereas scans on BASIS were performed in discrete temperature increments of about $3 \mathrm{~K}$. We also cannot rule out differences in the thermometry and note that the measurements on BASIS were performed after storing the hermetically-sealed sample at room temperature for a five-month period during which time the membrane could have annealed. Considering all of the possible sources of discrepancies, the similarities seen in the elastic and delta-function intensities are remarkable. 
Figure 2.7 also reveals similarities between the intensities of the narrow-Lorentzian component used to fit the wet (c) and dry (f) DMPG spectra. Upon cooling, the narrow-Lorentzian intensities were found to be nearly constant as a function of temperature and hydration level. Although the elastic scans show that the wet sample contains roughly 3.0 - 3.6 times the amount of water than the dry sample, the intensities associated with their narrow-Lorentzian components are equivalent, suggesting this type of water is common among the samples. Further evidence for a common water type in wet and dry DMPG comes from analysis of their quasielastic widths as a function of momentum transfer $Q$ (see Fig. 2.8).

\subsubsection{Modeling Water Diffusion}

Here, we analyze the translational diffusion of water at the interface with singlesupported DMPG membranes by means of the jump diffusion model, also known as the Chudley-Elliot model [27], in which water molecules undergo a stochastic process of 'jumping' from one site to another. The jump diffusion model is not limited to water, and can also be applied to other small molecules undergoing translational diffusion. The time elapsed between jumps is known as the residence time $\tau_{0}$, which describes the time needed for an individual water molecule to oscillate within a cage formed by neighboring water molecules. The expression for the jump-diffusion model can be written as

$$
\Gamma(Q)=\frac{D Q^{2}}{1+D Q^{2} \tau_{0}}
$$

where, $\Gamma$ is the half-width at half-maximum (HWHM) of a Lorentzian term representing translation motion, $D$ is the diffusion coefficient, $Q$ is the momentum transfer 
of the neutron, and $\tau_{0}$ is the residence time of the diffusing molecule. Taking the low $Q$ limit of Eq. 2.3 one arrives at linear expression known as, Fick's Law

$$
\Gamma(Q)=D Q^{2}
$$

Fick's Law can be an extremely useful method, particularly when only low $Q$ data is available, for determining diffusion coefficients of molecules. In this section, we will explore the capabilities and limitations of the two models in order to select the most reliable model which best describes the dynamics of the membrane-associated water observed in our DMPG samples.

The jump-diffusion model has been used for decades in fitting quasielastic neutron scattering spectra of many systems to elucidate molecular motions both translational and rotational diffusion $[28,29]$. At high momentum transfers $Q$ the model predicts a leveling off of the quasielastic width depending on the confinement geometry and residence time whereas the low momentum transfer region contains information on translational diffusion. It is important to understand that values of the HWHM $\Gamma$ are only reliable within certain energies defined by the instrumental resolution and dynamic range. For example, the Back Scattering Silicon Spectrometer (BASIS) [25] has an energy resolution of $\pm 3.5 \mu \mathrm{eV}$ (FWHM) and a dynamic range of $\pm 120 \mu \mathrm{eV}$ (FWHM). Therefore, neutrons that transfer energies less than or greater than these limits are considered unreliable. This restriction is particularly apparent at high $Q$ and high $T$ as the dynamics involved in our measurements can result in energy transfer order of hundreds of $\mu \mathrm{eV}$.

Figure 2.8 shows the $Q^{2}$ dependence of HWHMs obtained from Lorentzians used to fit the QENS spectra of wet and dry DMPG at $253 \mathrm{~K}$. The blue line in Fig. 2.8 was 


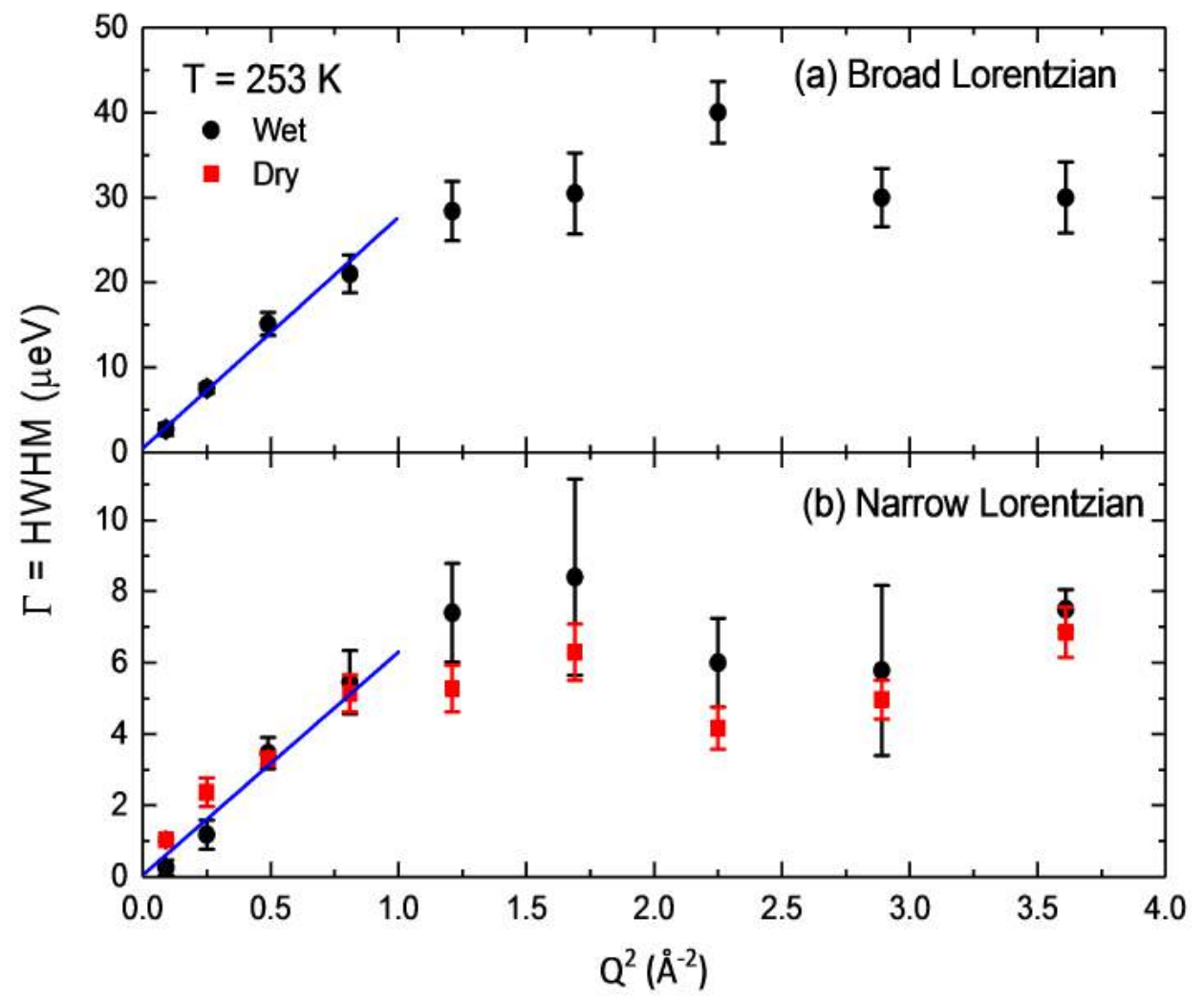

Figure 2.8: HWHM as a function of $Q^{2}$ for (a) the broad- and (b) the narrowLorentzian components in the QENS spectra for the wet and dry DMPG samples at $T=253 \mathrm{~K}$ as measured upon cooling. The blue line represents the linear fit to $Q$ values, $0.5 \AA^{-1}, 0.7 \AA^{-1}$, and $0.9 \AA^{-1}$ determined by Fick's Law (see Eq. 2.4) with a slope that is proportional to the diffusion coefficient of the water component represented by each Lorentzian. In panel (a), the slope inferred from the analysis of the broad-Lorentzian component yields a diffusion coefficient close in value to that of bulk supercooled water. From panel (b), we infer the same diffusion coefficient for water in the wet and dry samples represented by the narrow-Lorentzian component and termed confined 2. The resulting diffusion coefficients are plotted in Figure 2.11. 
obtained by fitting Fick's Law (Eq. 2.4) to $Q$ values: $0.5 \AA^{-1}, 0.7 \AA^{-1}$, and $0.9 \AA^{-1}$, plus the origin. The slope of the blue line is proportional to the diffusion coefficient whose units can be converted using the relationship, $1 \mu \mathrm{eV} \AA^{2}=1.519 \times 10^{-7} \mathrm{~cm}^{2} / \mathrm{s}$. There is evidence in Fig. 2.8 of a leveling off of the HWHM at high $Q$ for both the broad- and narrow-Lorentzian components as predicted by the jump diffusion model. To investigate how well a jump diffusion model describes the dynamics of the membrane-associated water compared to Fick's Law, we attempted to fit the halfwidth of Lorentzian components in our DMPG samples, representing the quasielastic scattering, with Eq. 2.3.

Figure 2.9 shows the results of fitting Eq. 2.3 to $\Gamma(Q)$ of four different Lorentzians used to fit the QENS spectra of wet DMPG for temperatures $T<253 \mathrm{~K}$. Recall, for $T>253 \mathrm{~K}$, two Lorentzians were required to fit the spectra in wet DMPG; however, below $253 \mathrm{~K}$ the narrow component of the QENS spectra vanishes, indicating the water participating in this motion froze out. From the fits in Fig. 2.9, we are able to extract temperature-dependent diffusion coefficients $D(T)$ and residence times $\tau_{0}$ for the water associated with wet DMPG. However, for temperatures $T>250 \mathrm{~K}$, we found that the values of the diffusion coefficient $D$ obtained by fitting Eq. 2.3 to the broad Lorentzian component of the wet DMPG sample and the narrow component of the dry sample were systematically higher and had larger error bars than those determined by assuming a linear dependence of the HWHM on $Q^{2}$ (Ficks law) at low $Q$. More importantly, the $D$ values obtained by fitting to Eq. 2.3 were also larger than those obtained for bulk supercooled water by QENS [30] and NMR [31]. Therefore, we limited our analysis of the residence times to temperatures $T<250 \mathrm{~K}$.

Figure 2.10 shows temperature-dependent residence times extracted by fitting wet 


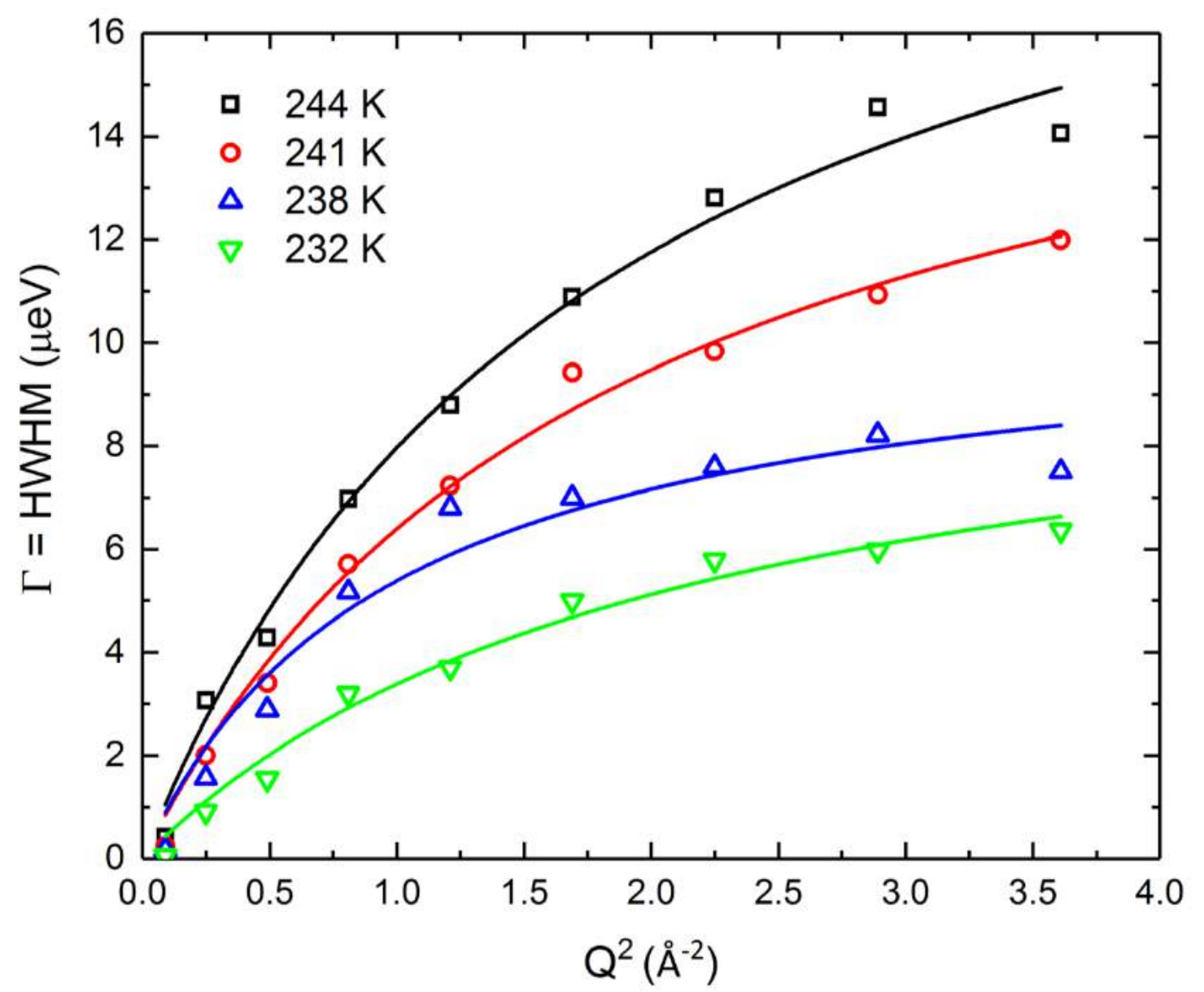

Figure 2.9: HWHM of the broad Lorentzian term used to fit the low temperature quasielastic spectra for wet DMPG. The solid color lines are fits to the corresponding data obtained using Eq. 2.3. 


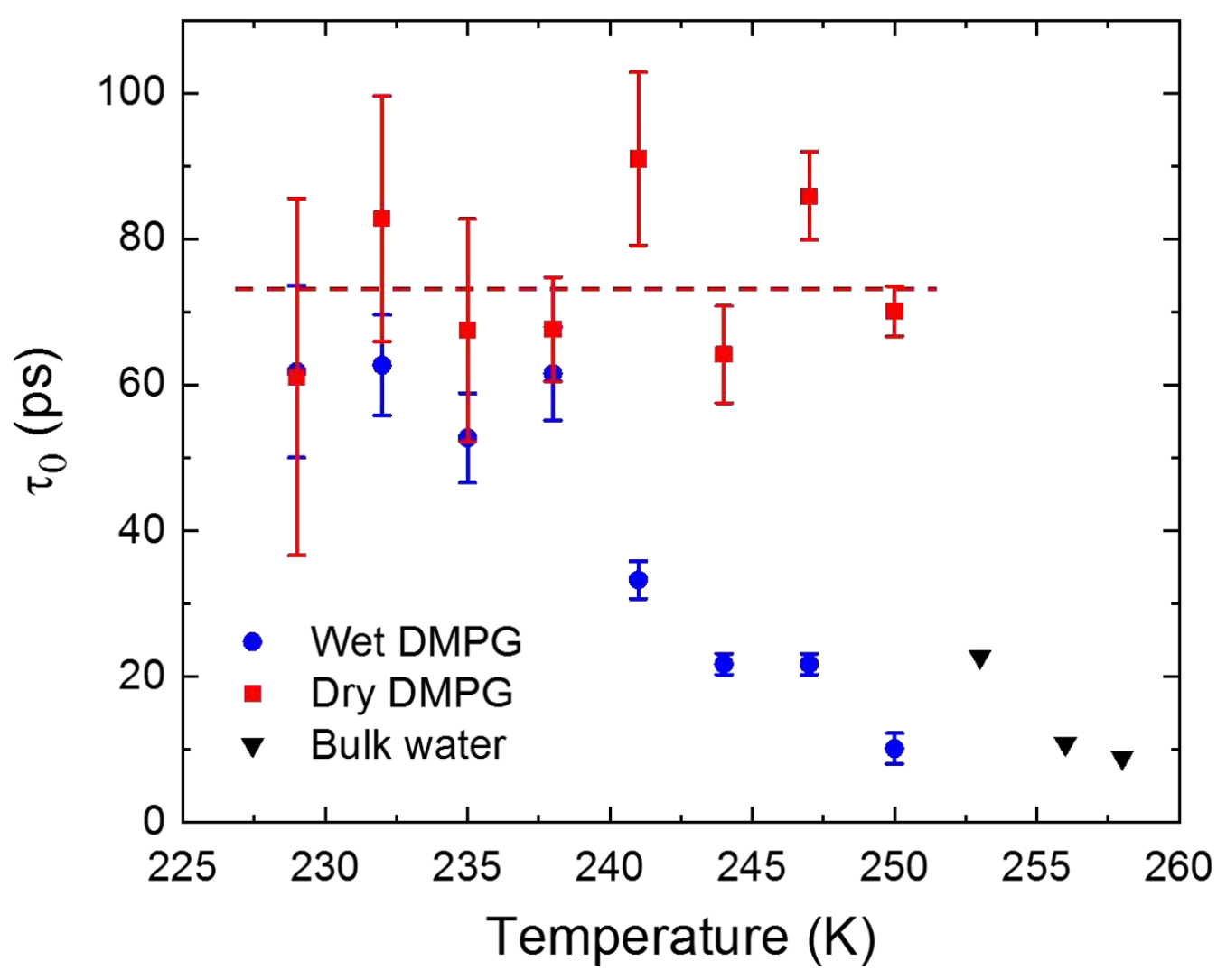

Figure 2.10: Low-temperature residence times $\tau_{0}$ for water associated with singlesupported DMPG membranes. The red and blue data points were calculated from the narrow and broad Lorentzian components used to fit the quasielastic spectra of the dry and wet samples, respectively. The black triangles are values for bulk supercooled water taken from Ref. [30]. By applying the jump diffusion model (Eq. $2.3)$ to the HWHM of the Lorentzian terms, we find good agreement between $\tau_{0}$ of wet DMPG and bulk supercooled water at $250 \mathrm{~K}$ whereas the dry sample possesses a weak temperature dependence of its $\tau_{0}$ with an average of $73 \mathrm{ps}$ as indicated by the horizontal dashed line. As the wet DMPG sample is cooled below $240 \mathrm{~K}$, we recover $\tau_{0}$ observed in the dry sample. 
and dry DMPG $\Gamma(Q)$ with Eq. 2.3. For comparison, Fig. 2.10 also includes residence times for supercooled bulk water obtained from Teixeira et al. 2.3. The $\tau_{0}$ inferred from the broad Lorentzian component in the spectra of the wet sample agrees well with that found for bulk water [30] at a temperature of $253 \mathrm{~K}$ and then rise steeply as the temperature is lowered. Analysis of the narrow Lorentzian component in the dry sample representing confined 2 water nearest the lipid head groups (cf. Fig. 2.2) yielded residence times with little temperature dependence but with relatively large error bars. This behavior reflects the weak temperature dependence and large error bars for the HWHM of the narrow-Lorentzian component in the spectra of the dry sample at high $Q$. For $T<250 \mathrm{~K}$, we obtain an average value of $\tau_{0}$ to be roughly 74 ps, close to the value to which the wet sample rises at $237 \mathrm{~K}$ as shown in Fig 2.10.

As mentioned previously, the greater energy transfers at high $T$ and $Q$ in our experiments prevents us from reliably using the jump diffusion model to fit $\Gamma(Q) \mathrm{s}$ associated with wet and dry DMPG for all temperatures measured. Therefore, to be consistent, Fick's Law was used to determine diffusion coefficients associated with water hydrating our model membranes, just as depicted by the blue line in Fig. 2.8.

\subsection{Water Dynamics Associated with DMPG}

In this section, we will use methods discussed in the previous subsection, Modeling Water Diffusion, to analyze quasielastic neutron scattering (QENS) spectra collected from wet and dry DMPG membranes on BASIS. Elastic neutron scans collected on the HFBS from wet and dry DMPG, shown in Fig. 2.4(a) and Fig. 2.4(d), respectively, are relevant for understanding results in this section as their freezing/melting features aid in the interpretation of the diffusion coefficients and associated water types revealed 
by QENS measurements. Although there are many differences in the behavior of water interacting with zwitterionic DMPC compared to anionic DMPG, there do exist similar water types defined by their diffusion coefficients and location from the membrane-water interface. Therefore, a comparison between water types associated with DMPC and DMPG membranes will also be discussed.

\subsubsection{Comparing characteristic water types in DMPC and DMPG membranes}

Figure 2.11 shows the temperature dependence of the diffusion coefficients $D$ for the wet and dry DMPG samples derived from the broad and narrow Lorentzian components of the QENS spectra. For completeness, values of $D$ obtained from wet and dry DMPC have also been included in Fig. 2.11 [4]. For comparison, diffusion coefficients for bulk water determined from QENS [30] and NMR [31] measurements are also shown. From $270 \mathrm{~K}$ down to $253 \mathrm{~K}$, we see that $D$ inferred from the broad-Lorentzian component of the wet sample agrees with values obtained for bulk supercooled water from the QENS and NMR measurements to within the experimental uncertainties. For example, at $253 \mathrm{~K}$, we find $D=0.44 \times 10^{-5} \mathrm{~cm}^{2} / \mathrm{s}$ (slope in Fig. 2.8(a)) compared to the values $D=0.47 \times 10^{-5} \mathrm{~cm}^{2} / \mathrm{s}$ determined for bulk supercooled water by NMR [31] and $D=0.42 \times 10^{-5} \mathrm{~cm}^{2} / \mathrm{s}$ by QENS [30]. However, at $295 \mathrm{~K}$ the value of $D$ inferred from the broad-Lorentzian component is somewhat less than that of bulk water, a discrepancy attributed by the FWHM of this Lorentzian near $Q=0.9 \AA^{-1}$ beginning to exceed the dynamic range of BASIS $(120 \mu \mathrm{eV})$.

In addition to comparing the temperature dependence of $D$ inferred from the broad Lorentzian component in the spectra of the wet DMPG sample with bulk supercooled 
water, we also compare it with that of two DMPC membranes with effective water thicknesses of $\sim 110 \mathrm{~nm}$ (wet) and $\sim 11 \mathrm{~nm}$ (dry), respectively [3]. At $270 \mathrm{~K}$, the diffusion coefficients inferred from the broad component of the wet DMPC and DMPG samples agree to within experimental error. This observation supports the conjecture that the broad-Lorentzian component represents the motion of bulk-like water above the membrane, which is largely unaffected by the underlying membrane.

For both DMPC and DMPG membranes, we identify the bulk-like water by a diffusion coefficient close to but somewhat less than that of bulk supercooled water at the same temperature. In the case of the DMPG sample, confined 2 water is characterized by a diffusion coefficient $D$, which has both a much smaller magnitude and a weaker temperature dependence than bulk-like water. In the case of the dry DMPG membrane, $D$ of the confined 2 water can be tracked over a wide temperature range from $295 \mathrm{~K}$ down to $220 \mathrm{~K}$. This behavior is consistent with continuous freezing of the confined 2 water over this wide temperature range.

For both wet and dry DMPC membranes, we identify confined 2 water as that which remains mobile below the freezing of bulk-like and confined 1 water. In Fig. 2.11 , we see that $D$ decreases to a nearly constant value in a narrow temperature range below $260 \mathrm{~K}$. It is noteworthy that at $260 \mathrm{~K}$ the confined 2 water in DMPC has $D \sim 4 \times 10^{-6} \mathrm{~cm}^{2} / \mathrm{s}$, which is about 4 times greater than that of confined 2 water in the DMPG sample. Unlike DMPG, we are unable to observe confined 2 water in coexistence with bulk-like water at higher temperatures in the QENS spectra of the DMPC samples. Also, it is difficult to quantify the amount of confined 2 water from the temperature dependence of the elastic intensity of the DMPC samples.

Because we find evidence of confined 1 water only below the freezing point of bulk- 


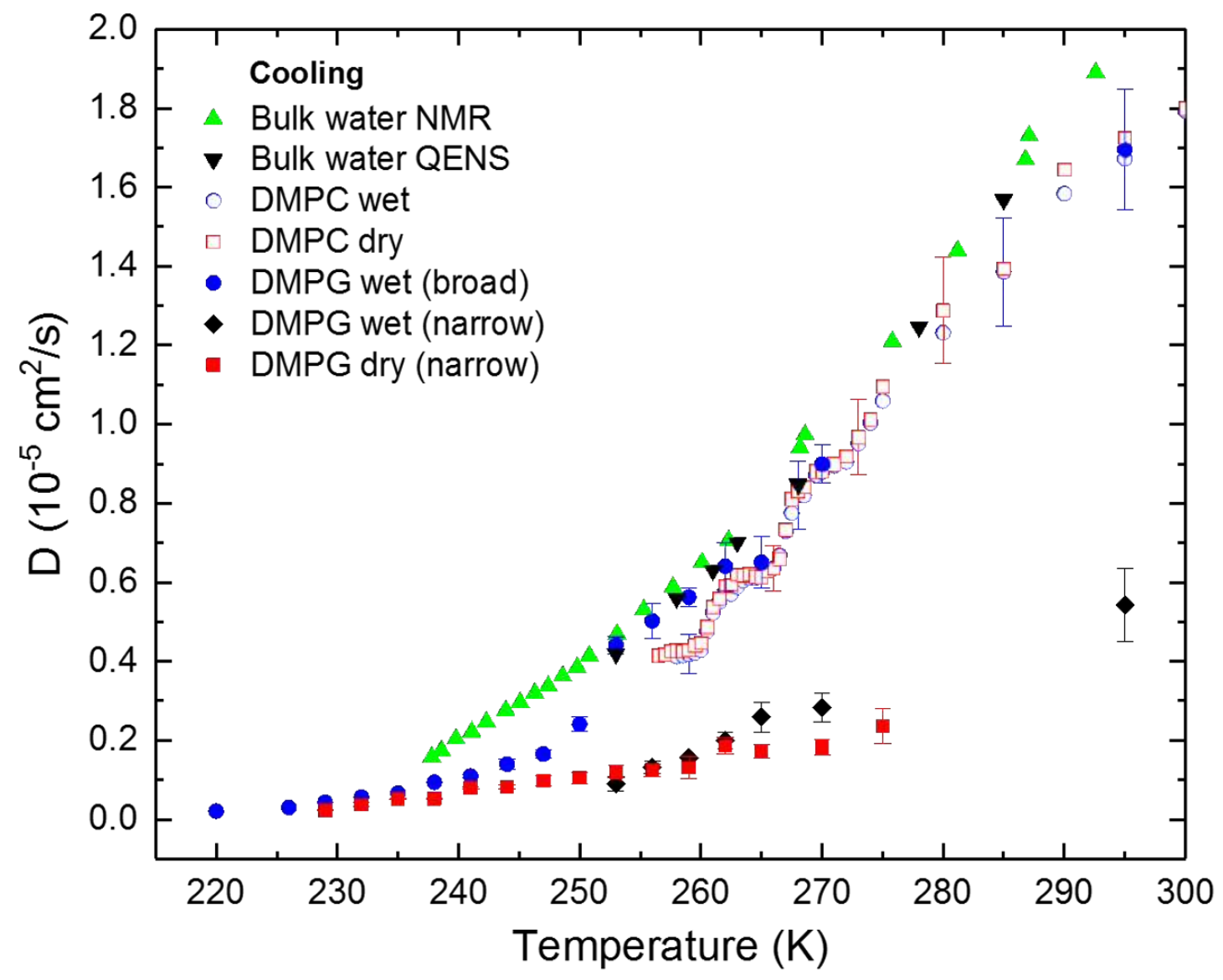

Figure 2.11: Diffusion coefficients $D$ inferred from the analysis of the QENS spectra of both wet and dry DMPG samples as a function of temperature on cooling. For comparison, the values of $D$ obtained for bulk-supercooled water are also shown from NMR (green up triangles) from Ref. [31] and QENS measurements (black down triangles) from Ref. [30]. The solid blue circles are obtained from the analysis of the broad-Lorentzian component of the wet sample and the black diamonds from its narrow-Lorentzian component. The solid red squares are from the analysis of the narrow-Lorentzian component of the dry sample. Also plotted are values of $D$ for two DMPC samples with a different water content: wet (open circles) and dry (open squares) with effective water thicknesses of $\sim 110 \mathrm{~nm}$ and $\sim 11 \mathrm{~nm}$, respectively. The value of the diffusion coefficient of the wet DMPG at $220 \mathrm{~K}$ was obtained from the analysis of the QENS spectra collected on HFBS. All other diffusion coefficients for the membrane-associated water were determined from spectra collected on BASIS. Values of $D$ and error bars were determined by a weighted least squares linear fit to $\Gamma(Q)$ vs. $Q^{2}$ (Fick's Law see Eq. 2.4) over $Q$ values: $0.5 \AA^{-1}, 0.7 \AA^{-1}, 0.9 \AA^{-1}$, plus the origin. 
like water and in a relatively large amount (effective thickness $\sim 23 \mathrm{~nm}$ ), we suggest it is confined to the region between bulk ice and the lipid head groups. Above the melting point of bulk ice, bulk-like and confined 1 water are believed to move on the same time scale and therefore would be indistinguishable in a QENS experiment. Based on the analysis of the DMPG QENS spectra, we posit that the slower-moving confined 2 water is localized within, and just outside the lipid head group region.

The DMPG and DMPC membranes only show evidence of confined 1 water on cooling to temperatures below the freezing of bulk-like water. Therefore, we have suggested that the freezing behavior and diffusion coefficient $D$ of confined 1 water may be determined by its proximity to overlying bulk ice as well as the lipid head groups beneath it. For the wet DMPG sample, this freezing behavior is characterized on cooling by the nearly linear increase of the elastic neutron intensity in temperature Region 2 of Fig. 2.5 and the steep decrease in $D$ in this temperature range (Fig. 2.11).

It is clear the dry DMPG sample has less bulk-like water; and, in fact, its broadLorentzian component, which was tentatively identified with bulk-like water above the membrane, was about a factor of 5 weaker than for the wet sample at $270 \mathrm{~K}$. Moreover, the broad-Lorentzian component of dry DMPG had about half the intensity of its narrow-Lorentzian component (see Fig. 2.7). For this reason, we were unable to determine a translational diffusion coefficient for the bulk-like water in the dry sample. However, it was possible to analyze the narrow-Lorentzian component in the dry DMPG sample over a wider temperature range than for the wet sample (see Fig. 2.11).

For $T>250 \mathrm{~K}$, the intensity of dry DMPG is comparable to that of the wet DMPG (see Fig. 2.7); and, for $T<250 \mathrm{~K}$, the bulk-like water is frozen out as discussed above 
so that only the narrow-Lorentzian component remains. In the restricted temperature range $253 \mathrm{~K}<T<263 \mathrm{~K}$, where $D$ can be determined from analysis of the narrowLorentzian component in both the wet and dry samples, we find agreement in $D$ to within the experimental uncertainty. It is evident in Fig. 2.11 that $D$ inferred from the narrow-Lorentzian component is less temperature dependent than that for the broad-Lorentzian component and for bulk supercooled water.

For the wet and dry DMPC samples, we identify confined 1 water from the temperature dependence of the elastic neutron intensity as the dominant contributor to the region of continuous freezing just below the step-like freezing transition of the bulklike water (see Fig. 2.3(a)). This identification is supported by the steep decrease in $D$ near $262 \mathrm{~K}$ for both the wet and dry DMPC samples (see Fig. 2.11).

The melting behavior of the membrane-associated water in the DMPG and DMPC samples also provides evidence of confined 1 water. In the wet DMPG sample, the melting of the confined 1 water is characterized by the substep in the elastic neutron intensity labeled $2 \mathrm{~h}$ in Fig. 2.3(b), which is followed by the melting of bulk-like water represented by a substep labeled $1 \mathrm{~h}$. Consistent with its smaller amount of confined 1 water, the dry DMPG sample does not have a substep in its elastic intensity on heating corresponding to the $2 \mathrm{~h}$ substep of the wet DMPG sample (cf. Fig. 2.3(b) and Fig. 2.4(d)).

Figure 2.12 shows the temperature dependence on heating of the diffusion coefficients determined from analysis of the broad and narrow-Lorentzian components of the wet DMPG sample and the narrow component of the dry DMPG sample. As expected, the major difference with the diffusion coefficients obtained on cooling (see Fig. 2.11) occurs near $237 \mathrm{~K}$ (see solid and open circles in inset to Fig. 2.12). In 


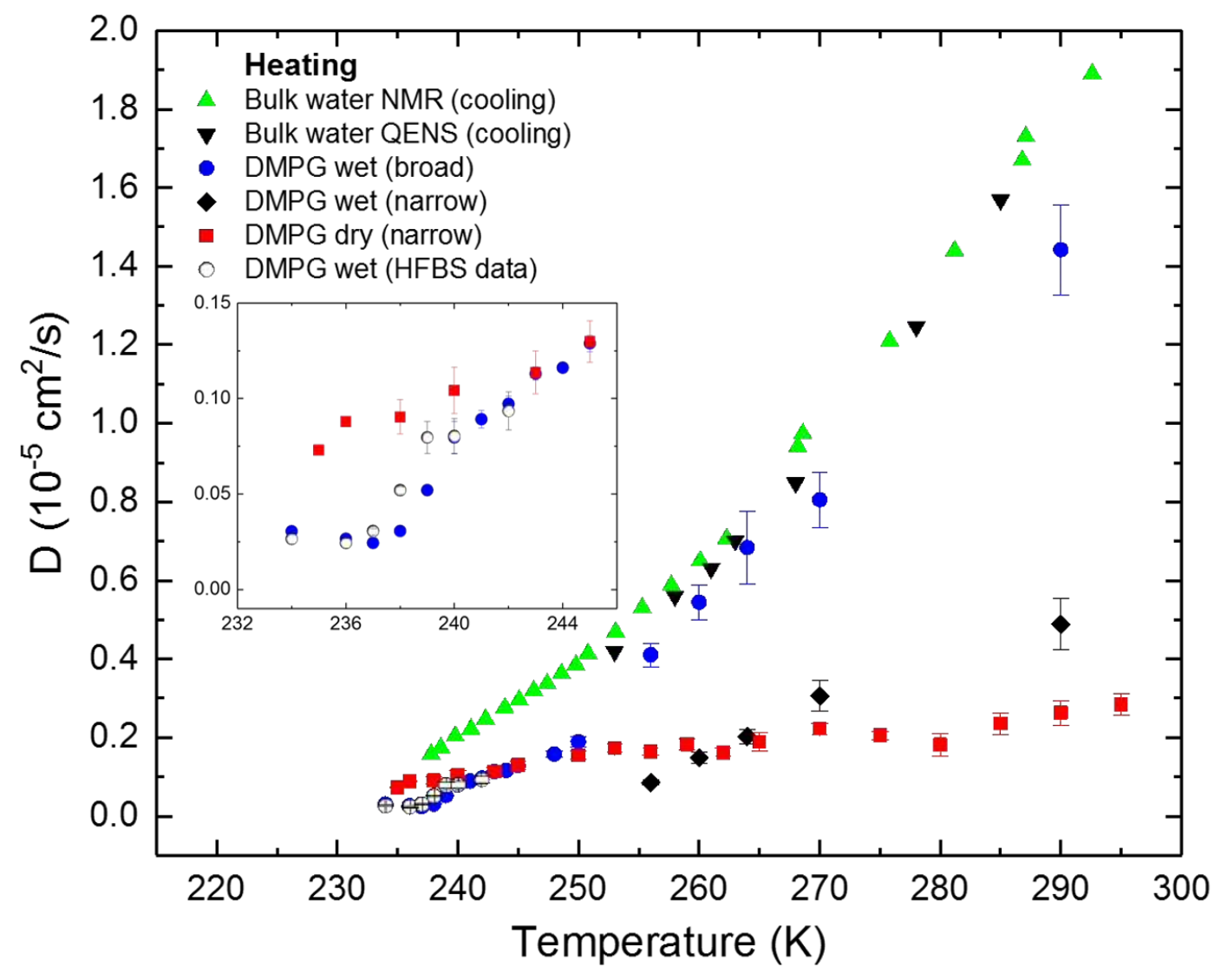

Figure 2.12: Diffusion coefficients $D$ inferred from the analysis of the QENS spectra of both wet and dry DMPG samples as a function of temperature on heating. Data points are coded as in Fig. 2.11. Inset shows low-temperature data on an expanded scale. Open black circles indicate data taken on the HFBS at NIST. All other diffusion coefficients were determined from spectra collected on BASIS. In both cases, HFBS and BASIS were able to produce $D$ values that reveal a heating substep at $238 \mathrm{~K}$, corresponding to the pre-melting of the membrane associated water observed in the elastic scans (see Fig. 2.4. Values of $D$ and error bars were determined by a weighted least squares linear fit to $\Gamma(Q)$ vs. $Q^{2}$ (Fick's Law see Eq. 2.4) over $Q$ values: 0.5 $\AA^{-1}, 0.7 \AA^{-1}, 0.9 \AA^{-1}$, plus the origin. 
this temperature range, the QENS spectra for the wet sample can be fit with a single Lorentzian that yields a diffusion coefficient $D$ showing an upward substep on heating, which appears neither for the dry sample nor for the wet sample on cooling. At higher temperatures $(T>240 \mathrm{~K})$, the diffusion coefficients of the wet and dry samples are similar to those obtained on cooling. In particular, both the wet and dry samples show evidence of a water component undergoing weakly temperature-dependent translational diffusion at a rate well below that of bulk supercooled water.

Similar QENS measurements were conducted upon heating using the HFBS in the temperature range where the melting substep near $240 \mathrm{~K}$ was observed in the wet DMPG sample (see Fig. 2.3(a)). The HFBS has a higher energy resolution (FWHM $\sim 1 \mu \mathrm{eV})$ and a smaller dynamic range (FWHM $\sim 36 \mu \mathrm{eV})$, which allow for a finer fitting of the narrow-Lorentzian component. We found strong similarities between the diffusion coefficients obtained from the narrow-Lorentzian component on the HFBS and BASIS instruments as shown in the inset of Fig. 2.12.

In conclusion, single-supported bilayers of the zwitterionic DMPC and anionic DMPG membranes provide useful model systems for comparing the structure and dynamics of their membrane-associated water. We have found the freezing/melting behavior and the translational diffusive motion of the membrane-associated water on nano length and time scales to be sensitive to the structure and charge of the lipid head group. Despite differences in their freezing/melting behavior and dynamics, the interfacial water in the zwitterionic and anionic membranes appear to share a classification scheme of water types based on the proximity of the water to the membrane: bulk-like, confined 1, confined 2, and bound water (see Fig. 2.2). Of these, confined 1 water has been identified only in coexistence with bulk ice and hence is 
not present at biologically relevant temperatures. It is expected that further study combining both QENS and molecular dynamic simulations will reveal greater detail of the water-lipid head group interaction and eventually the interaction of water with integral membrane proteins.

\subsubsection{Search for bound water in DMPG}

In our previous studies of wet and dry DMPC membranes [3], we found evidence of a weak narrow-Lorentzian component in their QENS spectra with a nearly $Q$ independent $\mathrm{HWHM} \sim 2.5 \mu \mathrm{eV}$, which is about a factor of 2 greater than the energy resolution on BASIS $(\mathrm{HWHM}=1.75 \mu \mathrm{eV})$. This narrow component was attributed to scattering from hydrogen nuclei in the lipid molecule and in water molecules bound to their head groups, all of which moved on the same nanosecond time scale. Therefore, we seek to determine whether there is also evidence of such bound water molecules in the spectra of the DMPG membranes.

Even though the QENS spectra represent an average over all of the membraneassociated water, the diffusive motion of water in the head group region of both membranes is sufficiently slower than that of bulk-like and confined 1 water that it has been possible to extract its contribution to the spectra. We find a difference between the DMPC and DMPG membranes in the type of diffusive motion of water in the head group region that is observable in their QENS spectra. For temperatures $T>270 \mathrm{~K}$ in DMPC, the dominant contribution to the observed QENS spectra by water in the head group region is from molecules that move on the same time scale as $\mathrm{H}$ atoms in the lipid, i.e., water molecules that we have referred to as "bound" [3]. This diffusive motion is nearly $Q$-independent $[3,7]$. In the case of DMPG, above 
$270 \mathrm{~K}$ the water in the head group region that contributes dominantly to the QENS spectra consists of confined 2 water, i.e., molecules undergoing translational diffusion at a rate faster than "bound" water but more slowly than the bulk-like and confined 1 water. We attribute this difference in water dynamics to the larger number of bound water molecules in DMPC compared to DMPG. From our QENS measurements on DMPC (Ref. [3]) in the gel phase, we estimated 7-10 water molecules bound per lipid, while $\sim 4$ per lipid has been determined by NMR [32]. These values are larger than the $\sim 2$ water molecules bound per lipid for DMPG in its gel-phase as inferred from our molecular dynamic simulations simulations [24].

Resolving a bound water component in the experimental QENS spectra was more difficult in the case of wet DMPG because of the presence of the narrow-Lorentzian component (HWHM $\sim 15 \mu \mathrm{eV}$ ) whose intensity is up to half that of the broadLorentzian component for $T>260 \mathrm{~K}$ (see Fig. 2.7). These two components would tend to mask a third Lorentzian component that is comparable in magnitude to the narrow-Lorentzian component and having a HWHM $\sim 2.5 \mu \mathrm{eV}$ as was the case for the bound water in the DMPC membrane. The bound water component should be easiest to observe at high temperature and high $Q$ where the broad-Lorentzian merges with the background scattering. We have found some evidence for a weak third Lorentzian in the QENS spectra of the dry DMPG sample at temperatures in the range $275 \mathrm{~K}$ to $290 \mathrm{~K}$; however, it is difficult to quantify its intensity and HWHM.

\subsubsection{Comparison with Molecular Dynamic Simulations}

Molecular dynamics (MD) is a powerful computational tool that has been used to investigate the structure and dynamics of water hydrating single freestanding bilayers 

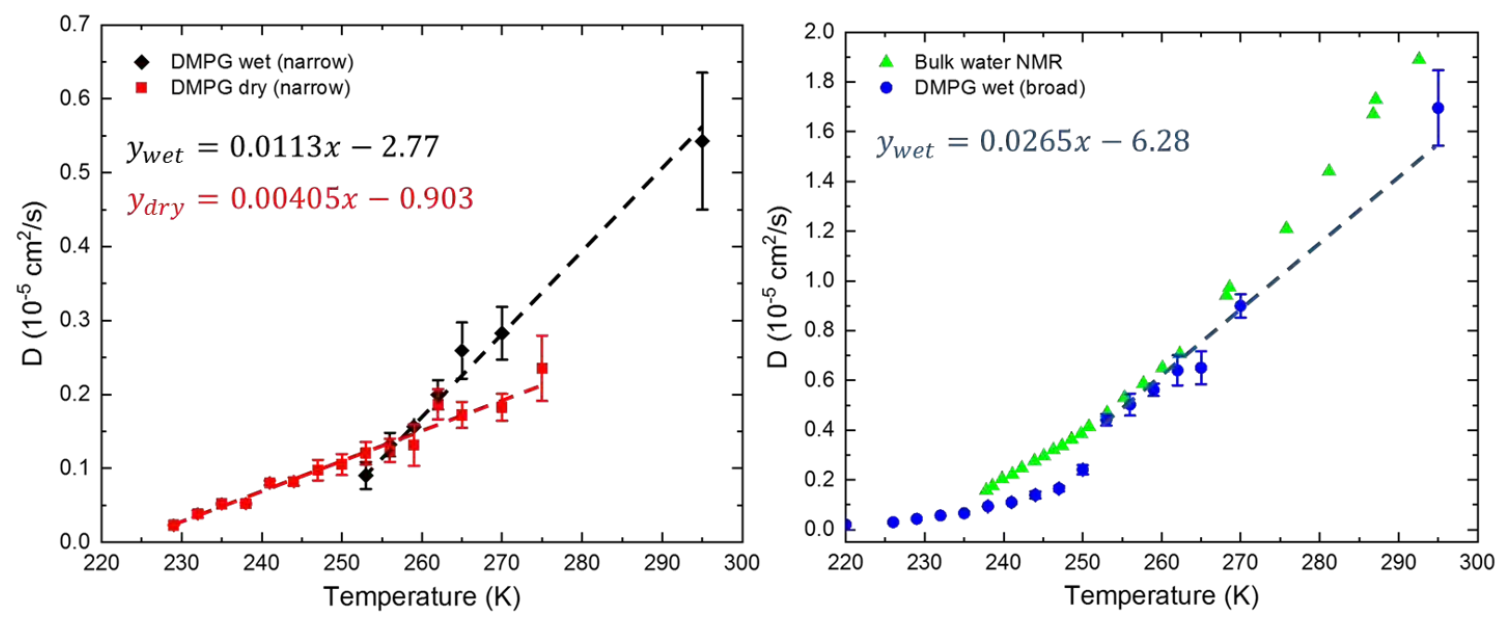

Figure 2.13: Extrapolation of diffusion coefficients inferred from narrow- (left) and broad- (right) Lorentzian components used to fit the dry and wet DMPG spectra. A weighted linear fit (colored dashed lines) was used to determine the equations displayed in the inset, which were later used in estimating diffusion coefficients at 310 K. Diffusion coefficients displayed here came from cooling data in Fig. 2.11.

[33-36]. Here we compare diffusion coefficients obtained from MD simulations with those of bulk-like water and confined 2 water inferred from analysis of the DMPG QENS spectra. MD simulations were performed at $310 \mathrm{~K}$ on a freestanding DMPG bilayer [24] (i.e. no substrate) similar to simulations reported earlier on a bare DMPC membrane [33]. Unfortunately, the simulations were unable to be performed in the low-temperature regime of the experiments due to their long equilibration times. Additionally, the limited dynamic range of HFBS and BASIS spectrometers $(<120$ $\mu \mathrm{eV})$ prevented reliable measurements at high temperatures. Therefore, in order to compare MD and experimental results, we have extrapolated our measured diffusion coefficients from temperatures as low as $230 \mathrm{~K}$ up to $310 \mathrm{~K}$, the temperature at which the simulations were performed.

Figure 2.13 shows results of extrapolating values of $D(T)$ determined experimentally by QENS to $310 \mathrm{~K}$, the temperature at which MD simulations were performed. 
Analysis of the broad-Lorentzian component measured in wet DMPG yields a diffusion coefficient of $D=1.7 \times 10^{-5} \mathrm{~cm}^{2} / \mathrm{s}$ at $295 \mathrm{~K}$ (see Fig. 2.11). Assuming a linear dependence over the temperature range where two Lorentzians were used to fit the wet DMPG spectra $(253 \mathrm{~K}<T<295 \mathrm{~K})$ and extrapolating $D$ to $310 \mathrm{~K}$, we estimate a high temperature-diffusion coefficient of $D=1.94 \times 10^{-5} \mathrm{~cm}^{2} / \mathrm{s}$. This extrapolation results in a slightly less value of $D$ compared to those inferred from the broad-Lorentzian component in the simulated spectra $\left(D=2.5 \times 10^{-5} \mathrm{~cm}^{2} / \mathrm{s}\right)[24]$. However, $D$ associated with wet DMPG at $295 \mathrm{~K}$ was determined from fits to values of $\Gamma(Q)$ that were beginning to exceed the dynamic range of BASIS. Therefore, it is possible this extrapolation underestimates the true value of $D$ at these temperatures.

In addition to the diffusion coefficients, MD simulations also determined the location of different water types defined by four $7.5 \AA$-thick slabs with their planes perpendicular to the membrane normal. Values of $D$ were compared with those obtained for confined 2 water from analysis of the narrow-Lorentzian component in the QENS spectra of dry DMPG, which presumably represents the dynamics of water closest to the lipid head groups. By linearly extrapolating diffusion coefficients inferred from the narrow-Lorentzian component in dry DMPG (red points in Fig. 2.13 ), we estimate $D \approx 0.353 \times 10^{-5} \mathrm{~cm}^{2} / \mathrm{s}$ at $310 \mathrm{~K}$. Applying the same method of extrapolation to the narrow-Lorentzian component belonging to the wet DMPG sample (black points in Fig. 2.13) we estimate $D \approx 0.733 \times 10^{-5} \mathrm{~cm}^{2} / \mathrm{s}$ at $310 \mathrm{~K}$. Since both narrow components are believed to be associated with confined 2 water both wet and dry DMPG, we average the two results for $D(T=310 \mathrm{~K})$ to arrive at $D=0.543 \times 10^{-5} \mathrm{~cm}^{2} / \mathrm{s}$. The averaged extrapolated $D$ was then compared to various water slabs identified in the MD simulations and was found to be in excellent 
agreement with the diffusion coefficient $\left(D=0.5 \times 10^{-5} \mathrm{~cm}^{2} / \mathrm{s}\right)$ calculated for the slab which has its inner boundary at the position of the phosphorous atom in the DMPG head group, supporting the claim that confined 2 water is in close proximity to the membrane.

The narrow-Lorentzian component in the fit to the simulated QENS spectra of DMPG is more difficult to reconcile with our experimental results. First, its intensity is very weak, corresponding to only $\sim 2.1$ water molecules per lipid, which is likely too small to be resolved in the observed spectra. Second, the width of the narrowLorentzian component in the simulated spectra does not show a $Q^{2}$ dependence at low $Q$, characteristic of translational diffusion. For this reason, the narrow-Lorentzian component in the simulations has been tentatively identified with water molecules bound to the lipid head group [24]. It is possible that at the higher simulation temperature, the slow translational diffusion of water in the head group region and motion of bound water are too close in time scale to be resolved.

Although there exist limitations preventing a direct comparison of the membraneassociated water dynamics in DMPG, indirect methods of analysis reveal several interesting similarities between experimental and computational results. 


\section{Chapter 3}

\section{Neutron Diffraction from Water}

\section{Hydrating Single-Supported}

\section{Zwitterionic and Anionic Lipid}

\section{Bilayers}

In this chapter, we will discuss results from measurements performed on the neutron diffractometer at the University of Missouri Research Reactor (MURR) located in Columbia, MO. Analysis of the integrated Bragg peak intensity as a function of temperature from water molecules hydrating single-supported lipid bilayers (SSLB) of zwitterionic DMPC and anionic DMPG reveal a different growth mode of ice crystals for the two membranes. These experiments were motivated by previous measurements of the incoherent elastically-scattered neutron intensities by Bai, Miskowiec et al. [3, 4], which showed qualitatively different freezing and melting behavior of the associated 
water for the two membranes (see Fig. 2.3). However, these measurements were unable to distinguish between the formation of crystalline and/or amorphous ice.

Elucidating the structure of membrane-associated water in DMPC and DMPG SSLBs requires the complementation of two different elastic neutron scattering techniques. The first involves incoherent elastic scattering of neutrons as measured on the High Flux Backscattering Spectrometer (HFBS), whose motivation and results for anionic DMPG membranes were previously discussed in Chapter 2. In this chapter, we will expand on these measurements, while also including results from DMPC. The second method involves the diffraction of neutrons from similarly prepared SSLBs and is the main focus of this chapter. Details regarding sample preparation of DMPC and DMPG were discussed in Chapter 1; however, it should be noted that samples made for neutron diffraction were hydrated with $\mathrm{D}_{2} \mathrm{O}$ rather than $\mathrm{H}_{2} \mathrm{O}$ in order to increase the coherent scattering from the ice.

\subsection{Introduction to Neutron Diffraction}

Neutron diffraction is a powerful tool for probing the atomic and magnetic structures of a wide variety of materials. An advantage for using neutrons as a scattering probe over other particles, such as a photon from an x-ray source, is the fact that neutrons do not posses a net electric charge and can therefore penetrate deep into matter where they experience weak interactions with atomic nuclei. In contrast, $\mathrm{x}-$ rays scatter from the electron clouds of atoms limiting their ability to detect lighter elements such as biologically relevant hydrogen, oxygen, and carbon. Furthermore, since the scattering mechanism of neutrons depend on the spin states of atomic nuclei, we are able to use isotopic substitution to reveal structural characteristics of a 
sample. Isotopic substitution is useful because of the vastly different coherent scattering cross-sections of deuterium (D) compared to hydrogen (H). Hydrogen has one of the largest incoherent scattering cross-sections of any known isotope [37], which produces a large background when performing coherent measurements such as diffraction. However, by substituting deuterium for hydrogen the incoherent background is greatly reduced, while simultaneously enhancing the signal produced by coherent scattering from deuterium. Therefore, the diffraction measurements were conducted with samples hydrated with $\mathrm{D}_{2} \mathrm{O}$ rather than $\mathrm{H}_{2} \mathrm{O}$.

Although the fundamental interaction of neutrons and x-rays with matter is very different, their elastic scattering processes are kinematically similar. For an elastic scattering event to occur, the energy (i.e. wavelength $\lambda$ ) of the incident and scattered particle must remain the same. That is, $k_{i}=k_{f}=k$ where $k$ is the wave vector magnitude of the particle and inversely proportional wavelength by,

$$
k=\frac{2 \pi}{\lambda}
$$

This allows us to express the magnitude of the momentum transfer $(Q)$ of the neutron, which is simply the difference between final $\left(\boldsymbol{k}_{f}\right)$ and initial $\left(\boldsymbol{k}_{i}\right)$ wave vectors as,

$$
Q=2 k \sin \theta=\frac{(4 \pi \sin \theta)}{\lambda}
$$

where $\lambda$ is the wavelength of the particle and $\theta$ is half of the scattering angle, $2 \theta$, which is the angle between $\boldsymbol{k}_{i}$ and $\boldsymbol{k}_{f}$ (see Fig. 3.1). One can easily derive Eq. 3.2 from the scattering triangle depicted in Fig. 3.1, assuming the magnitudes of initial and final wave vectors are equal (i.e. elastic scattering). 


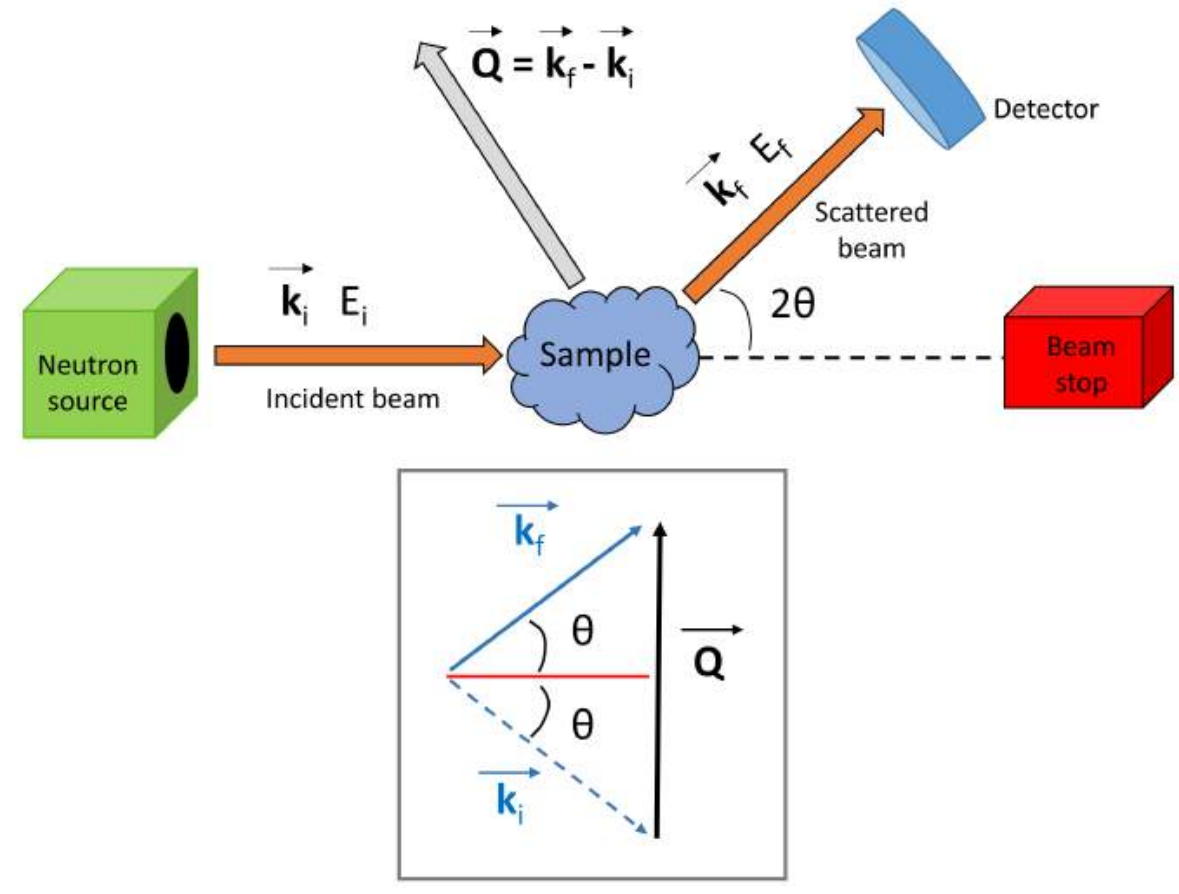

Figure 3.1: Cartoon (top) and vector diagram (bottom) representing the elastic scattering process. The momentum transfer, $\vec{Q}$, is the difference measured between wave vectors before $\left(\overrightarrow{k_{i}}\right)$ and after $\left(\overrightarrow{k_{f}}\right)$ a scattering event. In the case of elastic scattering, initial and final energies (i.e. wavelength) of the neutron are equal and only the direction of the scattered particle is affected by its interaction with the sample. Equation 3.2 can be derived from the scattering triangle displayed in the lower boxed diagram. 
Although the observables in a diffraction measurement are the intensities and scattered angles of the probing particles, details regarding the material structure can be extracted. An important parameter needed for solving the atomic structure of a material is the distance between scattering planes made up by the atoms within a sample. The expression relating distances between lattice planes within a crystal to particle wavelength and scattering angle is known as Bragg's Law,

$$
n \lambda=2 d \sin \theta
$$

where $n$ is a positive integer, $d$ is the distance between scattering planes, and $\theta$ is the angle of the incident beam measured with respect to the scattering plane (see Fig. 3.1). For the purpose of this experiment, we will only consider the first order reflections (i.e. $n=1$ ). Rearranging, solving Eq. 3.2 for $\lambda$, and substituting into Eq. (3.3) yields the relation $d=2 \pi / Q$, which can be more intuitive to consider since $d$ has units of length in real space. Bragg's Law will be used extensively to identify different diffraction peaks of crystallize water observed in our experiment.

\subsection{Experimental Setup and Operation}

The neutron diffractometer used in these experiments is located at the University of Missouri Research Reactor (MURR) in Columbia, MO. MURR is a 10 MW reactor, making it the most powerful university-operated research reactor in the United States. Located at MURR is a suite of four neutron scattering instruments: two powder diffractometers, a triple-axis spectrometer, and a reflectometer. The high reactor power, which translates to greater neutron flux, and variety of instrumentation make 
MURR a desired and accessible neutron scattering facility. In this section, we will discuss experimental setup, instrument capabilities, and operation of the neutron diffractometer used to elucidate membrane-associated ice structure in DMPC and DMPG.

\subsubsection{Instrumentation: Position Sensitive Detector (PSD) Diffractometer}

A photograph of the powder neutron diffractometer (PSD) is shown in Fig. 3.2. The PSD at MURR possesses a two-axis geometry with the first and second axes of rotation being the monochromator and sample position, respectively. The monochromator is the first and one of the most crucial components encountered by the neutron on its journey to detection. A beam of neutrons with a distribution of energies emerges from the reactor core and are incident on the monochromator, which is made up of 9 single-crystal silicon slabs in a vertically focusing configuration. The orientation of the monochromator is such that only neutrons with a selected wavelength in the thermal energy regime are reflected in the direction of the sample.

Upon its reflection from the monochromator, the neutron beam emerges from the beam port with a wavelength of approximately $1.485 \AA$ (37 meV) before being counted by a fission chamber also referred to as the monitor. Counting neutrons via the monitor is necessary as it provides a means for data normalization (i.e., the monitor count is proportional to the number of neutrons incident on the sample). Positioned just after the monitor, and before the sample, is a slit that can be adjusted in height and width depending on the size of the sample. Typically, a neutron beam with similar cross section dimensions as the sample is desired. Figure 3.3 shows images 


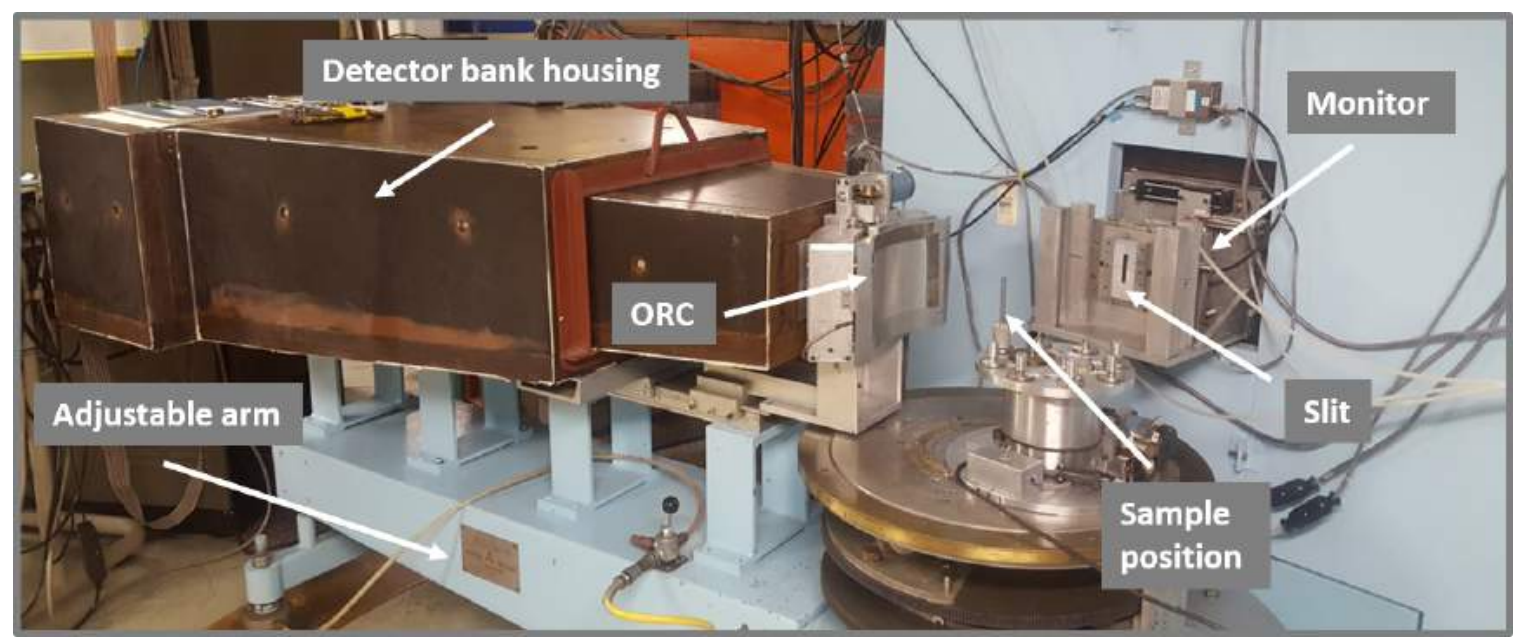

Figure 3.2: Photograph of the PSD instrument located on beam port D at MURR. Neutrons reflected from the Si monochromator (not shown) emerge from the beam port and are counted by a monitor before having its beam size defined by a slit. Neutrons that scatter from the sample in the direction of the detector bank encounter an oscillating radial collimator (ORC), which helps reduce background and unwanted scattering. Neutrons that traverse the ORC then travel through the opening in the masonite detector housing and are detected by one of five linearly position-sensitive detectors (not shown). The detector bank is mounted on a rotatable arm which can be moved to cover a $2 \theta$ range of approximately $5^{\circ}-105^{\circ}$.

of the neutron beam taken using a special scintillator camera at the sample position with and without the presence of a slit. The scattering and absorption of neutrons can occur simultaneously, while the sample is illuminated by the incident beam; however, only neutrons that are scattered into the solid angle subtended by the detectors will be counted. An example of neutron absorption at the sample position is shown by the dark regions in Fig. 3.3(b) and 3.3(c), which are the result of cadmium (Cd) strips placed onto a sample cell. Cd has one of the largest absorption cross sections of any known isotope (2520 barns for $25 \mathrm{meV}$ neutrons) [37], making it a common shielding material. The absorption of neutrons by $\mathrm{Cd}$ also releases potentially harmful gamma particles, therefore $\mathrm{Cd}$ is often complemented with other shielding materials such as 


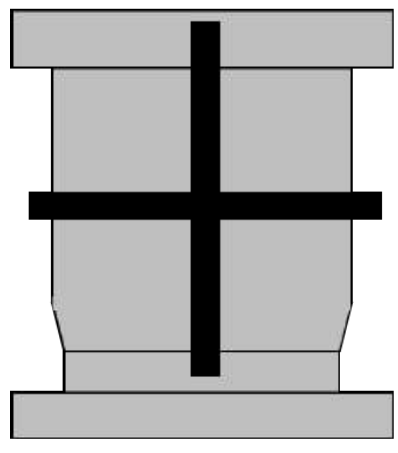

(a)

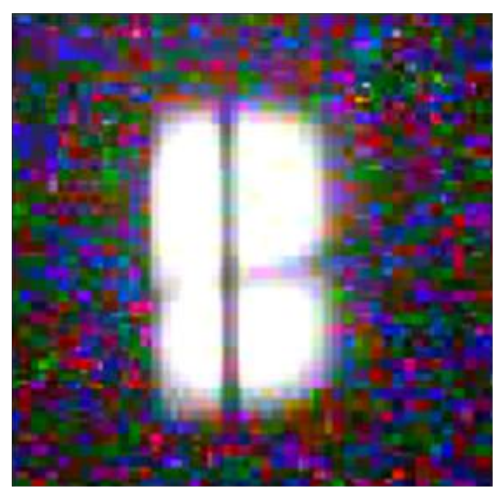

(b)

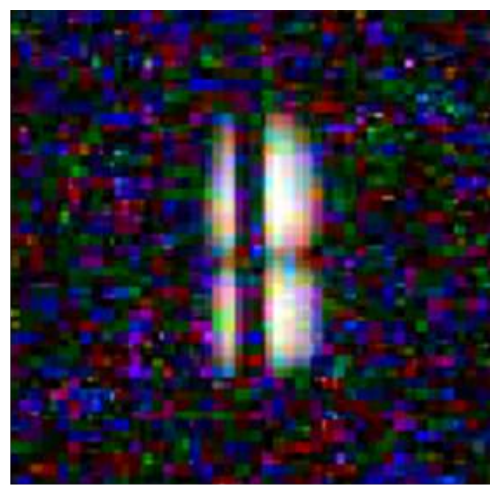

(c)

Figure 3.3: Cartoon representation of a diffraction cell at the PSD sample position (a) and photos of a neutron beam in the absence (b) and presence (c) of a slit with dimensions 2 inches high by 0.25 inches wide located after the monitor (see Fig. 3.2). The black cross in (a) represents strips of Cd placed on the sample cell (not to scale). A scintillator CCD neutron camera was placed in the straight through beam down stream from the sample position to capture images (b) and (c) with 3 second exposure times. The presence of the slit has a clear effect on the size of the neutron beam.

lead.

Neutrons scattered from the sample will first encounter an oscillating radial collimator (ORC) as they make their way to the detectors. The ORC is positioned just in front of the opening in the detector housing with the purpose of minimizing background counts as a result of additional scattering from peripheral objects such as heat shields and cryostat walls. The orientation and movement of the ORC will allow neutrons with trajectories originating from the sample position to traverse unaltered, while absorbing those beyond a minimum radius from the center off the sample.

The detector bank is made up of five cylindrical Position Sensitive Detectors (PSD), each filled with ${ }^{3} \mathrm{He}$ and arranged in a vertical plane, such that their cylindrical axis is oriented parallel to the ground. Each of the five detectors are approximately 1 meter long and stacked equally spaced vertically with the center of the middle 
detector positioned in the plane of the incident neutron beam. It follows an arc centered on the sample, resulting in a single arm position spanning roughly $20^{\circ}$ in

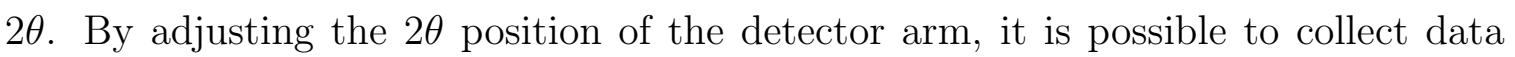
over a total angular range of $5^{\circ}<2 \theta<105^{\circ}$. Substituting these values into Eq. (3.2), we find the momentum transfers $Q$ of the PSD to range from $0.371 \AA^{-1}$ to $6.75 \AA^{-1}$, corresponding to $d$-spacings of $16.9 \AA$ and $0.931 \AA$, respectively. Because the detectors are linear, there is a software correction that determines $2 \theta$ for a given distance along the axis of the detector tube.

\subsubsection{Sample Environment and Control}

A well-controlled sample environment during the diffraction experiments was achieved using a liquid-nitrogen-cooled cryostat, courtesy of Brookhaven National Laboratory and nicknamed, the "Blue Dewar" (BD). In addition to the many upgrades of the PSD instrument, the BD also received several modifications that greatly enhanced its measurement capabilities, including the addition of high pressure gas lines for in situ measurements and the integration of a Lakeshore 336 cryogenic temperature controller and sensors [38]. Two temperature sensors were incorporated into the BD design: an uncalibrated $\mathrm{Pt}$ sensor located within the $\mathrm{Cu}$ cold finger used to control the setpoint temperature and a calibrated $\mathrm{Si}$ diode which can be mounted to the sample to measure its temperature.

The BD consists of two main sections, an upper and a lower. The upper section is made up of an inner reservoir and an outer jacket used to contain liquid nitrogen. Liquid nitrogen in the inner reservoir is circulated through a capillary to the copper cold finger to which the sample is mounted. The cooling power is regulated by 


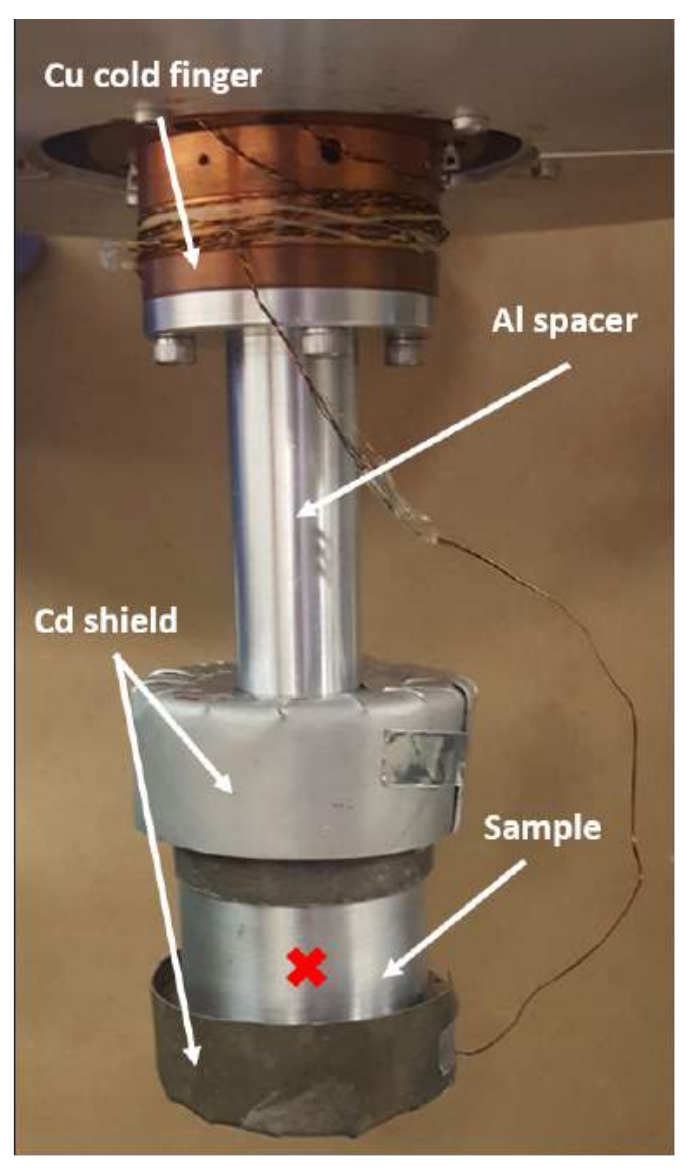

(a)

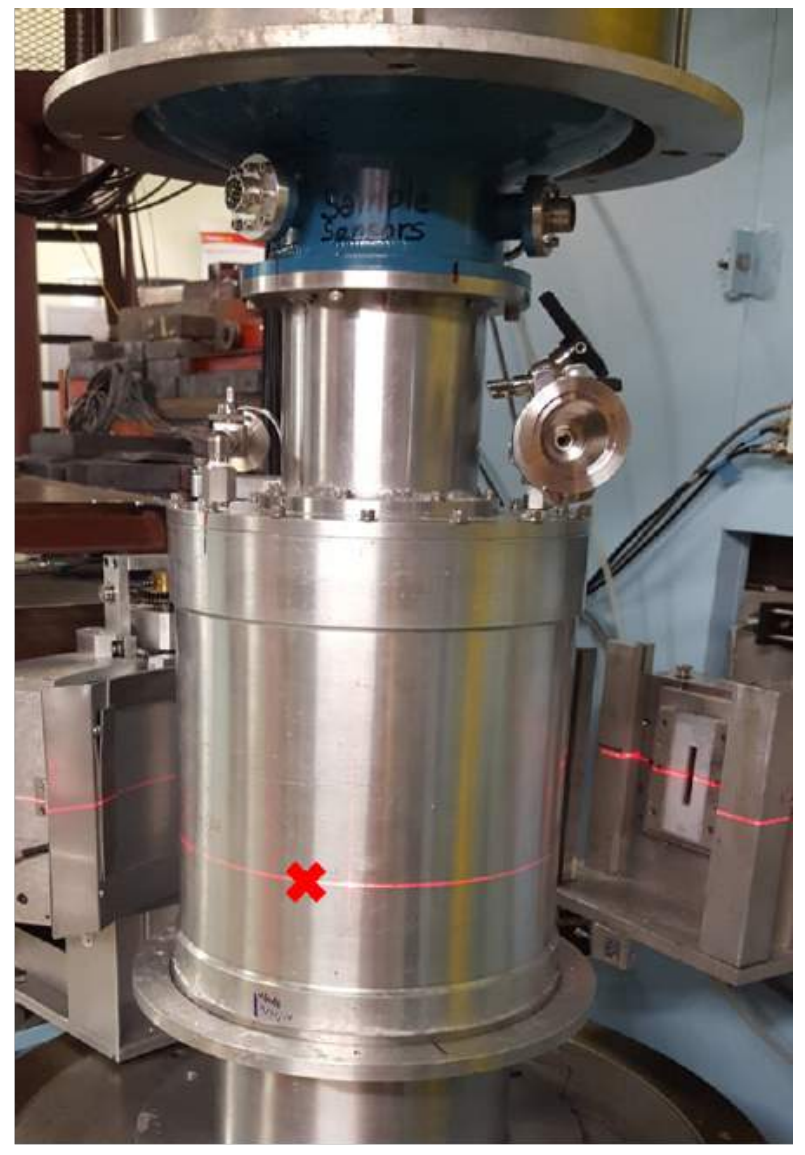

(b)

Figure 3.4: Photos of the diffraction sample mounted in the cryostat before (a) and after (b) being sealed and placed on the PSD. An Al spacer was required to mount the cell in order to center the sample in the neutron beam. A calibrated Si temperature sensor was fastened to the bottom of the sample and connected to the wire which encircles the $\mathrm{Cu}$ cold finger. Custom $\mathrm{Cd}$ shielding was placed around the upper and lower parts of the sample cell to prevent scattering from its thick Al base. The exposed cross-section of the sample is approximately 0.875 inches high by 2 inches wide. The red cross in (a) and (b) indicates the center of the sample, which aligns perfectly with the center line of the neutron beam represented by the horizontal red laser line in (b). 
adjusting a needle valve, which controls the flow rate of liquid nitrogen circulating through the cold finger. A delicate balance of the nitrogen flow rate and heater power output is required to achieve temperature stability of both $\mathrm{Pt}$ and $\mathrm{Si}$ sensors over the temperature range $160 \mathrm{~K}<T<300 \mathrm{~K}$. The role of the outer jacket is to cool the radiation shield, in addition to providing a heat shield for the inner reservoir. The outer jacket tends to evaporate rather quickly, therefore, it is regularly filled with liquid nitrogen over the duration of the diffraction experiment at a frequency of roughly $14-16$ hours.

The lower section of the BD is where samples of either DMPC or DMPG hydrated with $\mathrm{D}_{2} \mathrm{O}$ were mounted with their wafer reference edges oriented such that they faced the incident neutron beam. Photographs of the lower section setup are shown in Fig. 3.4 before (a) and after (b) it was isolated and placed on the PSD. To align the center of the sample with the neutron beam, an $\mathrm{Al}$ spacer of length 3.4 inches was placed between the $\mathrm{Cu}$ cold finger and the base of the sample cell. Sample cells used in the diffraction measurements are identical to ones from previous experiments described in Chapter 2. For additional information on sample cell design, the reader is referred to Appendix A.

Once mounted to the spacer, $\mathrm{Cd}$ masks were placed around the thick $\mathrm{Al}$ base and cap of the sample cell to minimize scattering from Al. The resulting available sample cross-sectional area left exposed to the neutron beam was approximately 0.875 inches high by 2.00 inches wide. Following attachment of the Cd masks to the sample cell, the Si temperature sensor was gently fastened to the bottom of the sample. Wires connecting the sensor were deliberately allowed to remain in open space as shown in Fig. 3.4(a) with their position being noted as discussed below. After the sample cell 
was secured and sensors fastened, the lower section of the BD was sealed, first with a radiation shield followed by the vacuum shroud in a concentric fashion. Fig. 3.4(b) shows the BD mounted on the PSD after the vacuum shroud was attached.

After hermetically sealing the lower section of the BD and placing it on the PSD, the cryostat was then rotated such that the wires connected to the Si sensor were positioned away from the incident neutron beam and outside any possible scattering trajectory that would lead to the PSD detectors. As mentioned earlier, the sample cell, which contains Si wafers, was also precisely positioned such that the reference edges of the wafers were oriented perpendicular to the incident neutron beam to minimize Bragg scattering from the single-crystal Si wafers. The orientation of the sample, its wafers, and temperature sensor components were replicated to the best of user ability for both DMPC and DMPG diffraction experiments.

After fixing sample and cryostat orientations, the BD chamber was evacuated using a diaphragm and turbopump from Pfeiffer Vacuum. Once a pressure on the order of $5 \times 10^{-6}$ Torr $\left(6 \times 10^{-9}\right.$ atm $)$ was established, the BD was isolated and vacuum station removed. A vacuum of this magnitude was found to result in a negligible temperature gradient between Pt and Si sensors, while also aiding in their stability over long periods of time. Temperatures measured between the two sensors during DMPC data collection $(250 \mathrm{~K}<T<275 \mathrm{~K})$ yielded a gradient $\leq 1 \mathrm{~K}$ whereas the cooler temperatures measured for DMPG $(200 \mathrm{~K}<T<250 \mathrm{~K})$ resulted in gradients as low as $0.1 \mathrm{~K}$.

Figure 3.5 illustrates the long-time temperature stability during several diffraction scans upon cooling. Near a temperature of $270 \mathrm{~K}$, Si and Pt sensors display a constant temperature gradient of about $1 \mathrm{~K}$, which is impressive considering the 


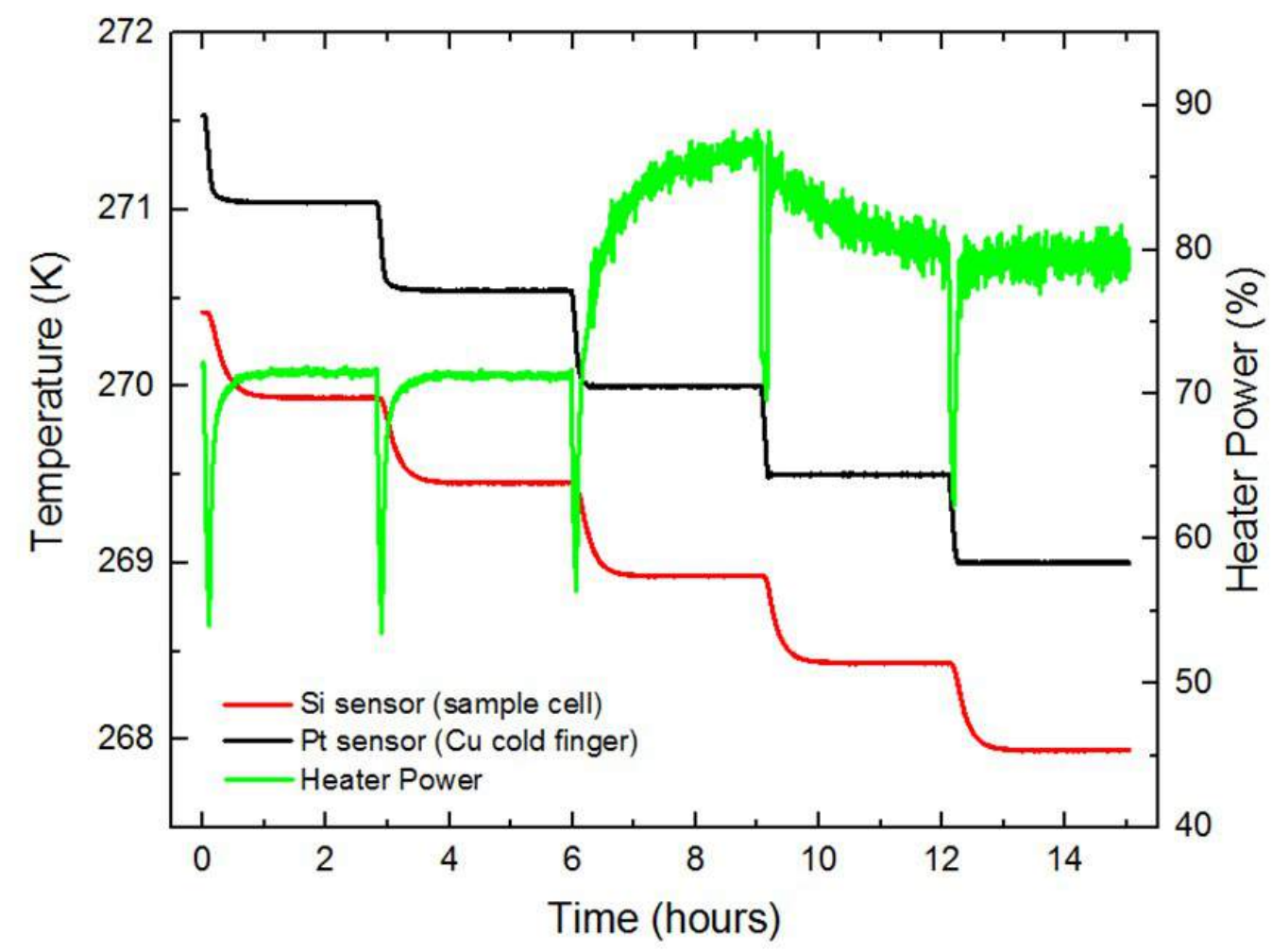

Figure 3.5: Stability of temperature sensors and heater power measured upon cooling of DMPC. Data collection was initiated once the Si sensor began leveling off, typically on the order of 1 hour after the temperature set point had been lowered by $0.5 \mathrm{~K}$ as indicated by steps in the black curve. The variation in heater power observed at the 6-hour mark was due to an adjustment made on the cooling flow valve with stabilization occurring soon after. Sharp dips in heater power (green curve) indicate the time at which temperature set points were changed. 
large distance ( $>6$ inches) and thermal mass between them. However, an increase of the gradient can still occur due to outgassing into the vacuum space during the time it takes to complete an experiment $(3-4$ weeks). Therefore, the vacuum space would periodically be pumped usually during reactor shut down, which occurs once a week for approximately 18 hours. The sharp dips in heater power (green curve in Fig. 3.5) indicate times at which the temperature setpoint of the Pt sensor was changed. Typically, once the Pt sensor reached its setpoint, it would take an additional 20 - 30 minutes for the Si sensor to equilibrate at which time data collection was initiated.

\subsection{Results of Neutron Diffraction Measurements}

To minimize the incoherent background while simultaneously increasing signal from crystalline ice associated with our membranes of DMPC and DMPG, heavy water $\left(\mathrm{D}_{2} \mathrm{O}\right)$ was used to hydrate the samples as opposed to $\mathrm{H}_{2} \mathrm{O}$. Volume amounts of $\mathrm{D}_{2} \mathrm{O}$ used to hydrate the membranes for diffraction measurements were the same (i.e., $120 \mu \mathrm{l})$ as volumes of $\mathrm{H}_{2} \mathrm{O}$ used in obtaining elastic scans from similar membranes collected on the HFBS. By hydrating DMPC and DMPG membranes with the same amounts of water as was used for runs on the HFBS, a direct comparison between freezing and melting curves in the elastic scans (see Fig.2.3) can be made to various crystal structures detected in the diffraction measurements.

A monitor count of 4 million neutrons per detector arm position was used to collect the diffraction date. On average the PSD monitor detects 600 neutrons per second emerging from the beam port, resulting in a total measurement time of roughly 1.8 hours per arm position, which subtends $20^{\circ}$ in $2 \theta$. Taking into account the time required to equilibrate temperatures between runs and data collection at 5 arm posi- 
tions, a full $2 \theta$ scan at a single temperature would take about 10 hours to complete. Therefore, it was necessary to focus on data collection at just one or two arm positions that would be capable of capturing the growth of ice Bragg peaks, while also allowing reasonable measurement times.

A detector arm position of $30^{\circ}$ was chosen to collect the majority of diffraction patterns because of the location of several intense hexagonal $\mathrm{D}_{2} \mathrm{O}$ peaks predicted to be within its range. Figure 3.6 shows a theoretical neutron diffraction pattern from an isotropic powder of hexagonal $\mathrm{D}_{2} \mathrm{O}$. The pattern was generated using the Inorganic Crystal Structure Database (ICSD) assuming a neutron wavelength of 1.5 $\AA$, close to the PSD neutron wavelength of $1.485 \AA$. The first five Bragg peaks in Fig. 3.6 possess the greatest intensities and conveniently all lie between $20^{\circ}$ and $40^{\circ}$ in $2 \theta$, making $30^{\circ}$ a well-suited angle to measure hexagonal ice growth.

It was unknown at first whether hexagonal $\mathrm{D}_{2} \mathrm{O}$ would be the only ice structures to form or if it was possible for cubic and/or amorphous phases to be present. Therefore, to assist in the identification of all ice Bragg peaks in the membrane samples, preliminary high-temperature complete full $2 \theta$ scans $(\sim 295 \mathrm{~K})$ were subtracted from low temperature scans, revealing the sole formation of hexagonal ice. Intensities from hexagonal $\mathrm{D}_{2} \mathrm{O}(100)$ and (110) (both with peak positions between $20^{\circ}<2 \theta<40^{\circ}$ ) were the dominant signals in both DMPC and DMPG diffraction patterns, justifying data collection at an arm position of $30^{\circ}$.

In addition to identifying the sole presence of hexagonal ice, the different patterns between low- and high-temperature scans also revealed that crystallite basal planes were parallel to the membrane plane. That is, only Bragg peaks with Miller indices of the form $(h, k, 0)$ were observed. 


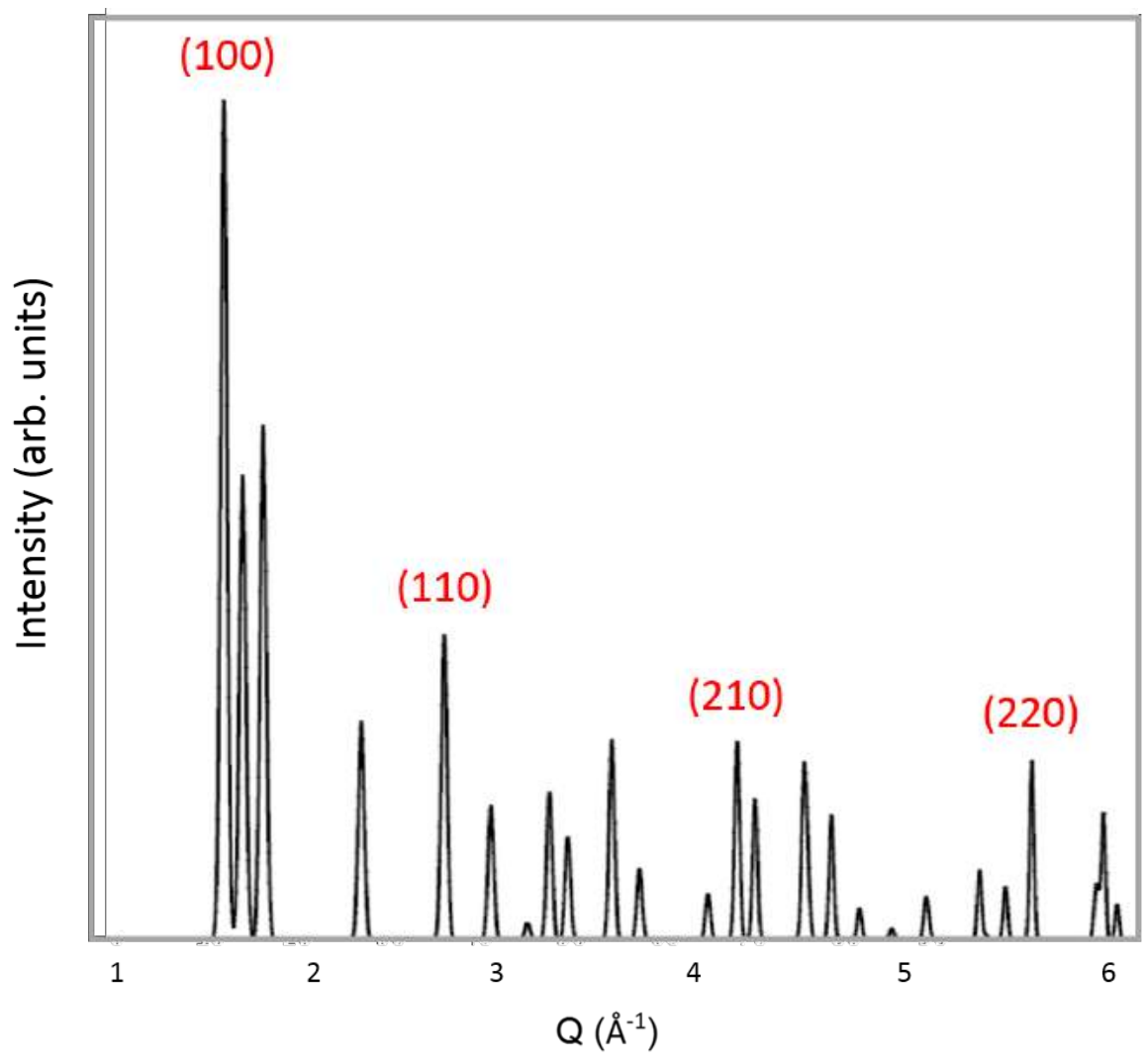

Figure 3.6: Theoretical neutron diffraction pattern of isotropic hexagonal $\mathrm{D}_{2} \mathrm{O}$ powder generated using the Inorganic Crystal Structure Database (ICSD) assuming a neutron wavelength of $1.5 \AA$. The four most intense Bragg peaks found in our membrane samples have been identified in the above figure by their Miller indices.

\subsubsection{Data Analysis and Interpretation}

Neutron diffraction patterns from DMPC and DMPG membranes were collected upon cooling and heating, with scans over the entire full $2 \theta$ range being performed at the highest and lowest temperatures of the experiment. Additional full scans were also collected at intermediate temperatures as time allowed. Miller indices for each hexagonal ice peak in our diffraction patterns were identified from their positions and known $d$-spacings inferred from values generated for an isotropic powder (see Fig. 
3.6). Parameters such as peak positions and half-width-half-maximum (HWHM) were determined by fitting neutron diffraction patterns that had their intensities summed over all 5 detectors.

Hexagonal $\mathrm{D}_{2} \mathrm{O}$ Bragg peaks were fitted using a Gaussian function of the form,

$$
y=y_{0}+\frac{A}{\omega \sqrt{\pi / 2}} \exp \left[-2 \frac{\left(x-x_{c}\right)^{2}}{\omega^{2}}\right]
$$

where, $y_{0}$ is the background offset, $x_{c}$ is the center peak position, $\omega$ is the width of the curve, and $A$ is the area determined by integrating over the width of the curve. The width $\omega$ can also be expressed as the full-width-at-half-maximum (FWHM) by the following equation,

$$
F W H M=\omega \sqrt{2 \ln (2)}
$$

An example of fitting a hexagonal $\mathrm{D}_{2} \mathrm{O}$ Bragg peak using Eq. 3.4 is shown in Fig. 3.7. Fits such as these were performed on each Bragg peak in the diffraction patterns for all temperatures measured. The resulting parameters were then analyzed in order to reveal any temperature-dependent similarities between the of Bragg peaks and previously measured elastic intensities.

Peak area parameters were the primary focus of analysis as they best represent the total elastic intensity from crystalline ice as opposed to peak heights. Therefore, areas obtained from fitting hexagonal $\mathrm{D}_{2} \mathrm{O}$ Bragg peaks in DMPC and DMPG were plotted as a function of temperature and compared with cooling and heating curves from similar samples hydrated with $\mathrm{H}_{2} \mathrm{O}$ measured on the HFBS. The resulting data sets reveal strong correlations between features in the HFBS elastic scans and the 


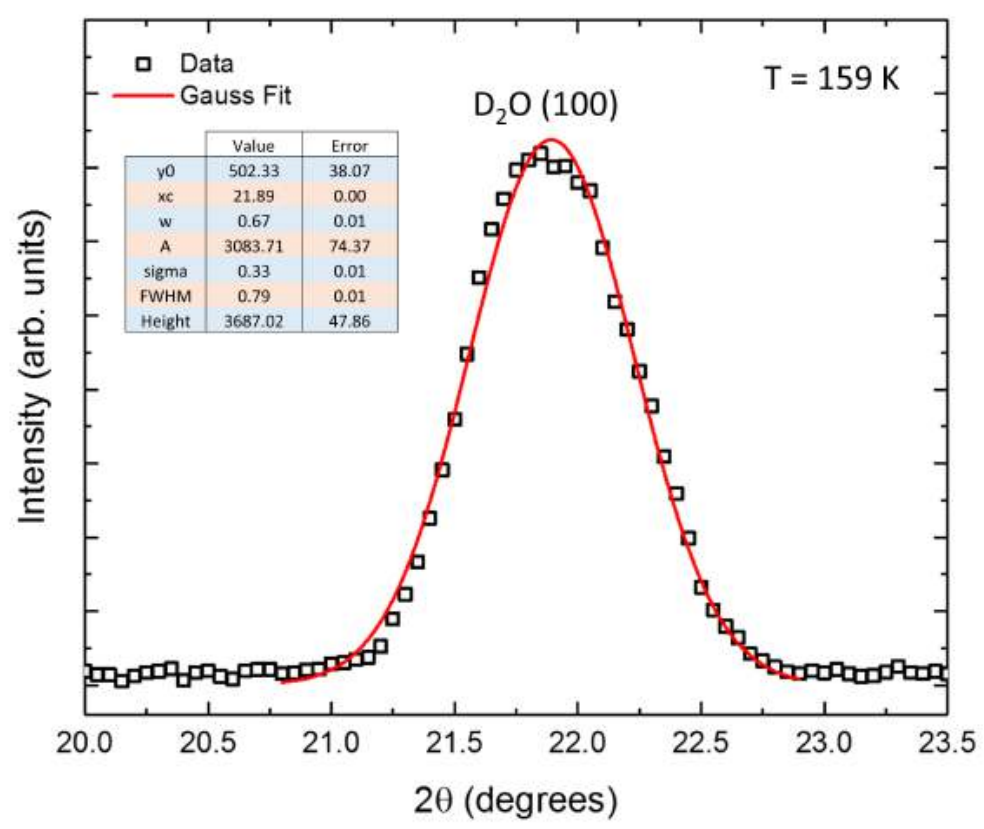

Figure 3.7: Gauss fit (red line) to the $\mathrm{D}_{2} \mathrm{O}$ hexagonal (100) Bragg peak measured at $159 \mathrm{~K}$ upon cooling DMPG. The Gaussian function used to fit the data is given by Eq. (3.4) with the resulting parameters tabulated in the inset. Fits were conducted using the raw intensity data summed over all 5 detectors.

freezing/melting behavior of hexagonal ice, which will be discuss in greater detail in the following sections.

Relative intensities of hexagonal $\mathrm{D}_{2} \mathrm{O}$ Bragg peaks were also of interest as they provide a more complete understanding of the crystalline ice structure. Therefore, ratios of the Bragg peak intensities were calculated and compared to theoretical values of an isotropic hexagonal $\mathrm{D}_{2} \mathrm{O}$ powder. Deviations of the relative intensity ratios were found for some $\mathrm{D}_{2} \mathrm{O}$ peaks, suggesting these ice crystallites could be strained in some way. The strain of the hexagonal ice crystallites can also be deduced from their lattice constants. Röttger et al. have shown using x-ray diffraction that the lattice constants of bulk isotropic powders of $\mathrm{H}_{2} \mathrm{O}$ and $\mathrm{D}_{2} \mathrm{O}$ decrease nearly linearly upon 
cooling, indicating a compression in the hexagonal basal plane [39]. We compare this linear trend of bulk hexagonal $\mathrm{D}_{2} \mathrm{O}$ lattice constants with those found in our membrane samples as inferred from their Bragg peak positions.

\subsubsection{Water Structure Associated with Supported Anionic DMPG Membranes}

Analysis of the Bragg peak intensity as a function of temperature has allowed us to correlate the formation of hexagonal crystalline ice growing on our model membranes to the freezing/melting behavior displayed by the hydration water from elastic neutron scans on the HFBS (see Fig. 2.3(b)).

Figure 3.8 shows a full neutron diffraction pattern from DMPG membranes hydrated by $\mathrm{D}_{2} \mathrm{O}$ collected on the PSD at MURR. The pattern in Fig. 3.8 was obtained by subtracting the full pattern collected from $295 \mathrm{~K}$ (room temperature) from the full pattern collected at $260 \mathrm{~K}$. This subtraction reveals five hexagonal $\mathrm{D}_{2} \mathrm{O}$ Bragg peaks with Miller indices: (100), (110), (210), (300), and (220). All Bragg peaks observed have a Miller indices of the form $(\mathrm{hk} 0)$, indicating that the $\mathrm{D}_{2} \mathrm{O}$ hexagonal crystals are oriented with their basal plane parallel to the membrane. As indicated previously, neutron diffraction measurements on the PSD are extremely time consuming, with full diffraction patterns taking as long as 15 hours to collect. Therefore, we limit our analysis of the integrated Bragg peak intensities to the two most intense Bragg peaks: the (100) and the (110).

Figure 3.9 displays a series of diffraction patterns collected at a detector arm position of $30^{\circ}$. This waterfall-style plot gives some insight into the temperature

dependence of the growth of hexagonal $\mathrm{D}_{2} \mathrm{O}$ crystals on a DMPG membrane. The 


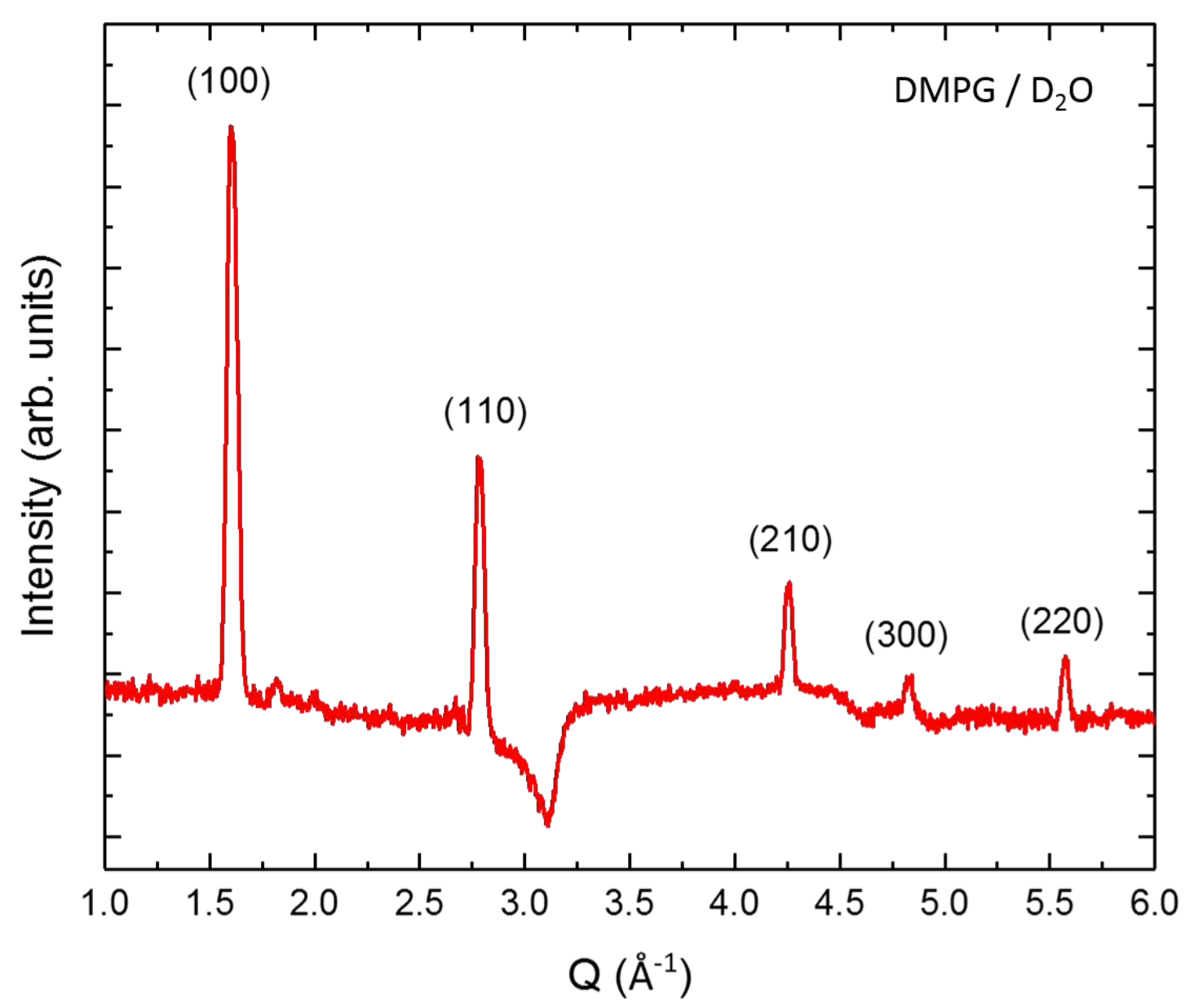

Figure 3.8: Full neutron diffraction pattern of hexagonal $\mathrm{D}_{2} \mathrm{O}$ hydrating DMPG membranes collected at $159 \mathrm{~K}$ measured on the PSD at the University of Missouri Research Reactor. A total of five hexagonal $\mathrm{D}_{2} \mathrm{O}$ peaks were observed in the DMPG membrane with their Miller indices indexed in the figure. Data were collected using 4 million neutron monitor counts and summed over all 5 detectors. A scan at $295 \mathrm{~K}$ has been subtracted as background. 


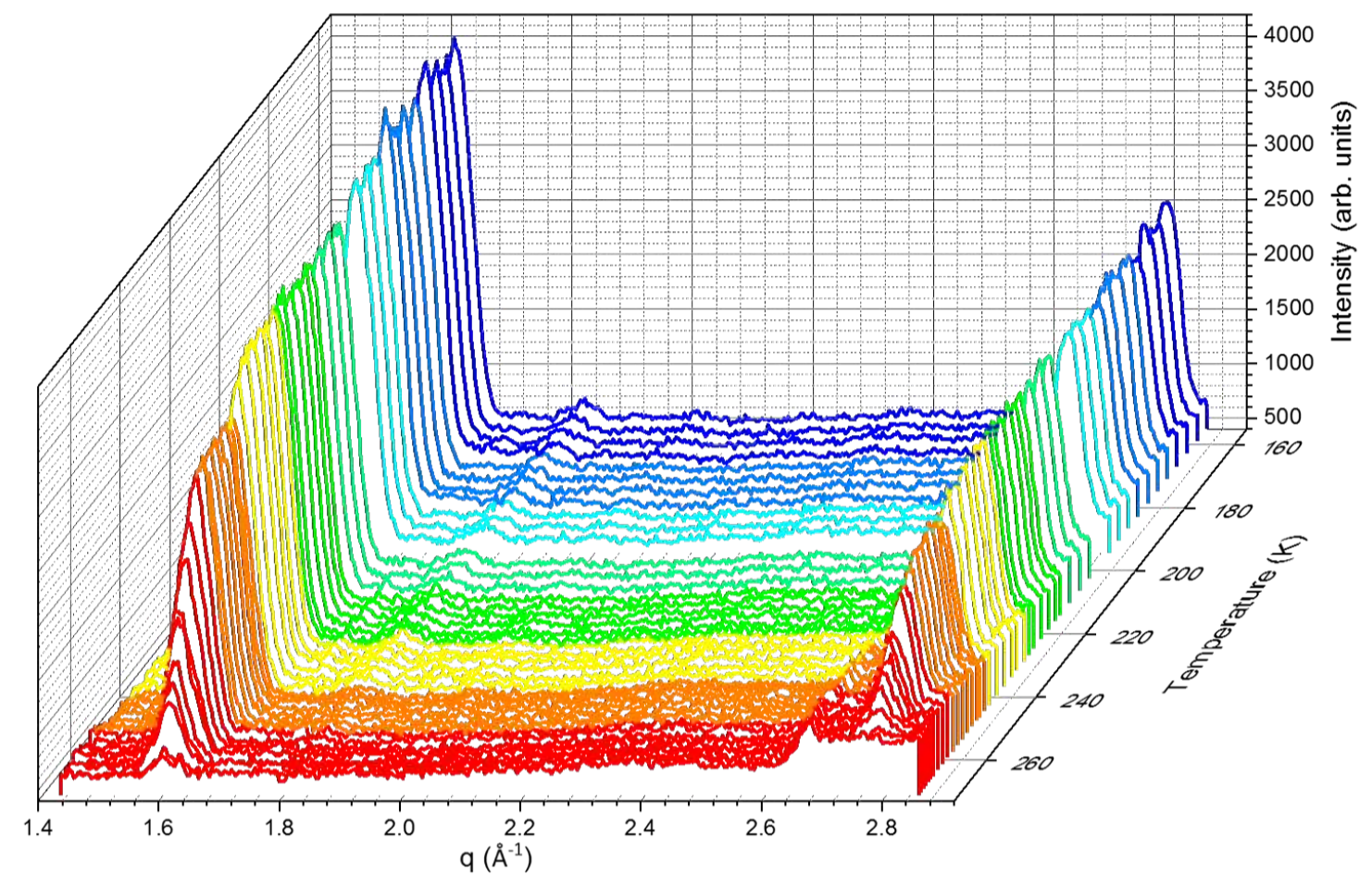

Figure 3.9: Waterfall plot of diffraction patterns as a function of temperature collected on the PSD from DMPC membranes hydrated with $\mathrm{D}_{2} \mathrm{O}$. The region of $Q$ plotted here represents the accessible angles the PSD detectors can span while at one arm position.

two most intense peaks in the waterfall plot shown in Fig. 3.9 are the (100) and (110), which are the Bragg peaks that are later analyzed in order to obtain integrated intensities as a function of temperature. These integrated intensities will then be compared to the incoherent elastic neutron intensities collected on the HFBS (see. Fig. 2.3(b)).

Figure 3.10 shows the results of integrating the Bragg peak intensities from hexagonal $\mathrm{D}_{2} \mathrm{O}$ observed in the DMPG membranes to the elastic neutron scans collected on the HFBS. Recall that the HFBS samples were hydrated with $\mathrm{H}_{2} \mathrm{O}$ whereas the 


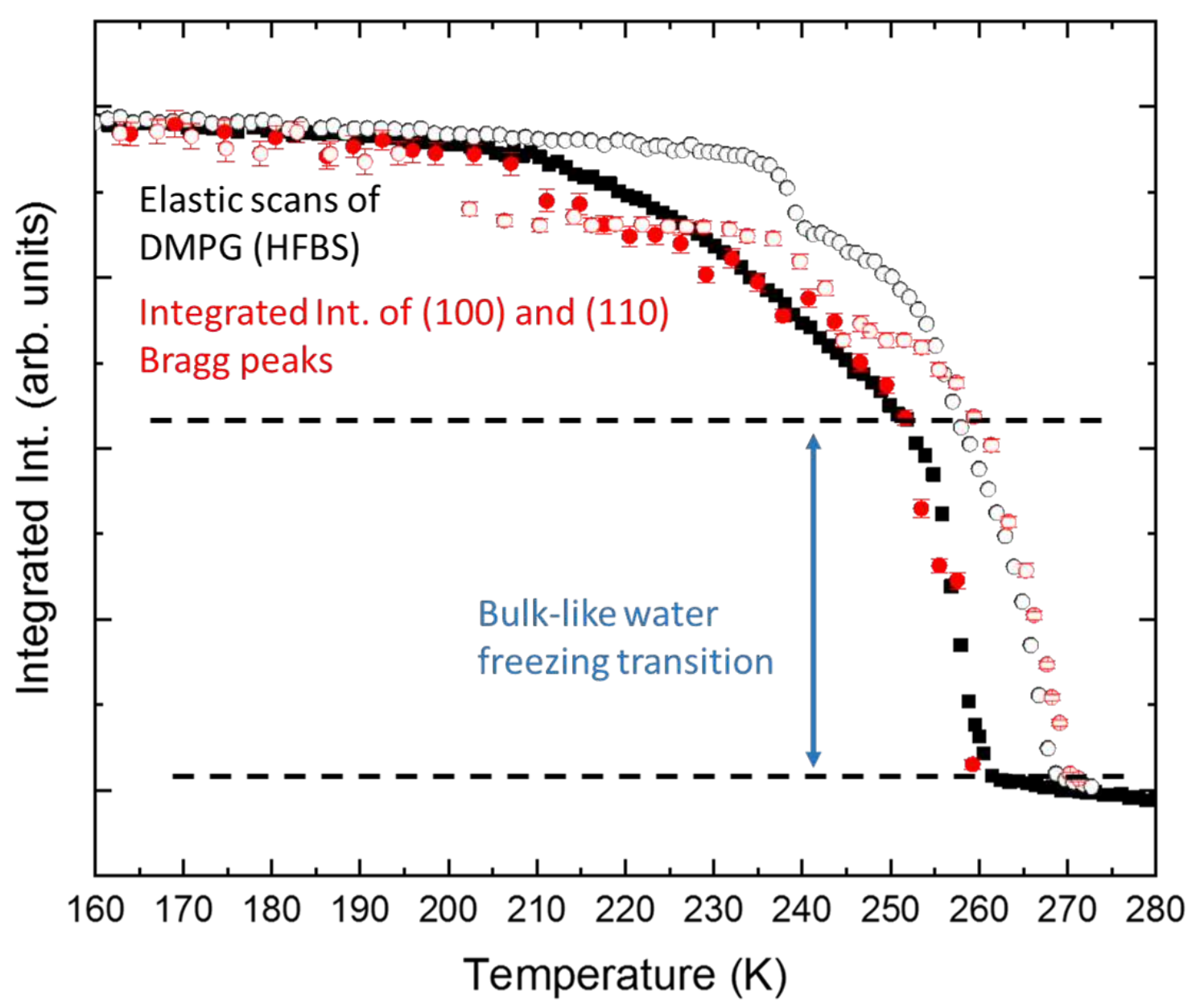

Figure 3.10: Cooling (closed symbols) and heating (open symbols) data from elastic neutron scattering measurements collected on the HFBS (black points) and the PSD (red points) from DMPG membranes hydrated with $\mathrm{H}_{2} \mathrm{O}$ and $\mathrm{D}_{2} \mathrm{O}$, respectively. The black data points have been taken from Fig. 2.3(b). The red data points have been obtained by summing the integrated intensities of the (100) and (110) Bragg peaks using Eq. 3.4. 
diffraction samples were hydrated with $\mathrm{D}_{2} \mathrm{O}$. Because the beam size on the HFBS and PSD are similar (i.e. similar scattering volumes) and each sample received similar volume amounts of hydration, we assume that the same amount of bulk-like water is present in the two samples. Under this assumption, we scale the two data sets shown in Fig. 3.10 by forcing their initial change in intensity to be the same. We justify this assumption based on the diffusion coefficients obtained from QENS measurements (see Chapter 2) on DMPG membranes in the same temperature region as the initial rise in intensity displayed in the elastic scans. By using this scaling factor, we find that the two data sets agree upon cooling (red circles and black squares in Fig. 3.10). The scaling is indicated by a vertical blue double arrow in Fig. 3.10. The agreement between data sets suggests that all the water hydrating DMPG membranes freezes into a hexagonal crystal structure with its basal plane parallel to the membrane surface.

Upon heating DMPG membranes hydrated with $\mathrm{D}_{2} \mathrm{O}$, we observe a series of substeps in the integrated Bragg peak intensity, just as was observed in the elastic intensity upon heating (see Fig. 2.3(b)). Moreover, there appears to be a premature melting step in the Bragg peak intensity at $200 \mathrm{~K}$, not seen in previous DMPG membranes hydrated with $\mathrm{H}_{2} \mathrm{O}$. This additional melting step could be a result of differences in the measurement. Recall, the diffraction scans require one to sit at a single temperature for the duration of the diffraction measurement, where as the measurements on the HFBS are performed over a continuous temperature ramp rate. If the ice is in a metastable state, sitting at one temperature for long periods of time could cause the ice to melt. Interestingly, we are able to recover the heating substep at $240 \mathrm{~K}$ (see open red circles in Fig. 3.10). Lastly, the Bragg peak intensity at higher temper- 
atures closely tracks the data obtained from the HFBS before completely melting at $270 \mathrm{~K}$, further supporting that all water hydrating DMPG membranes freezes into a hexagonal structure.

To get a better understanding for why hexagonal ice melts at such low temperatures when interacting with DMPG membranes, we analyze the Bragg peak position as a function of temperature upon cooling and compare it to bulk behavior. Figure 3.11 compares the temperature dependence of $\mathrm{D}_{2} \mathrm{O}$ peak positions from those reported by Röttger et al. [39] and ones measured by neutron diffraction from single-supported DMPG bilayers. For the weakest intense Bragg peaks, there is very little deviation from bulk values. However, when comparing the two most intense Bragg peaks, the (100) and (110), we find that around $200 \mathrm{~K}$ the peak positions begin to deviate from bulk values.

The linear trend of the bulk $\mathrm{D}_{2} \mathrm{O}$ peak position upon cooling in Fig. 3.11 implies that there is a uniform contraction of the hexagonal lattice. However, we do not see this trend in the positions of $\mathrm{D}_{2} \mathrm{O}$ Bragg peaks (100) and (110), suggesting that there could be some strain on the lattice at these lower temperatures. This strain is believed to be a result of the electrostatic landscape near the DMPG head groups, which perturbs the polar water molecules. We see a similar effect in the peak positions upon heating.

Figure 3.12 shows the Bragg peak positions upon heating from the four most intense $\mathrm{D}_{2} \mathrm{O}$ peaks. At low temperatures we see that the two most intense Bragg peaks do not change their position as a function of temperature until the sample is heated to $200 \mathrm{~K}$, which is the same temperature where the initial step in the heating curve is observed (see open red circles in Fig. 3.10). Once heated above $200 \mathrm{~K}$, the 


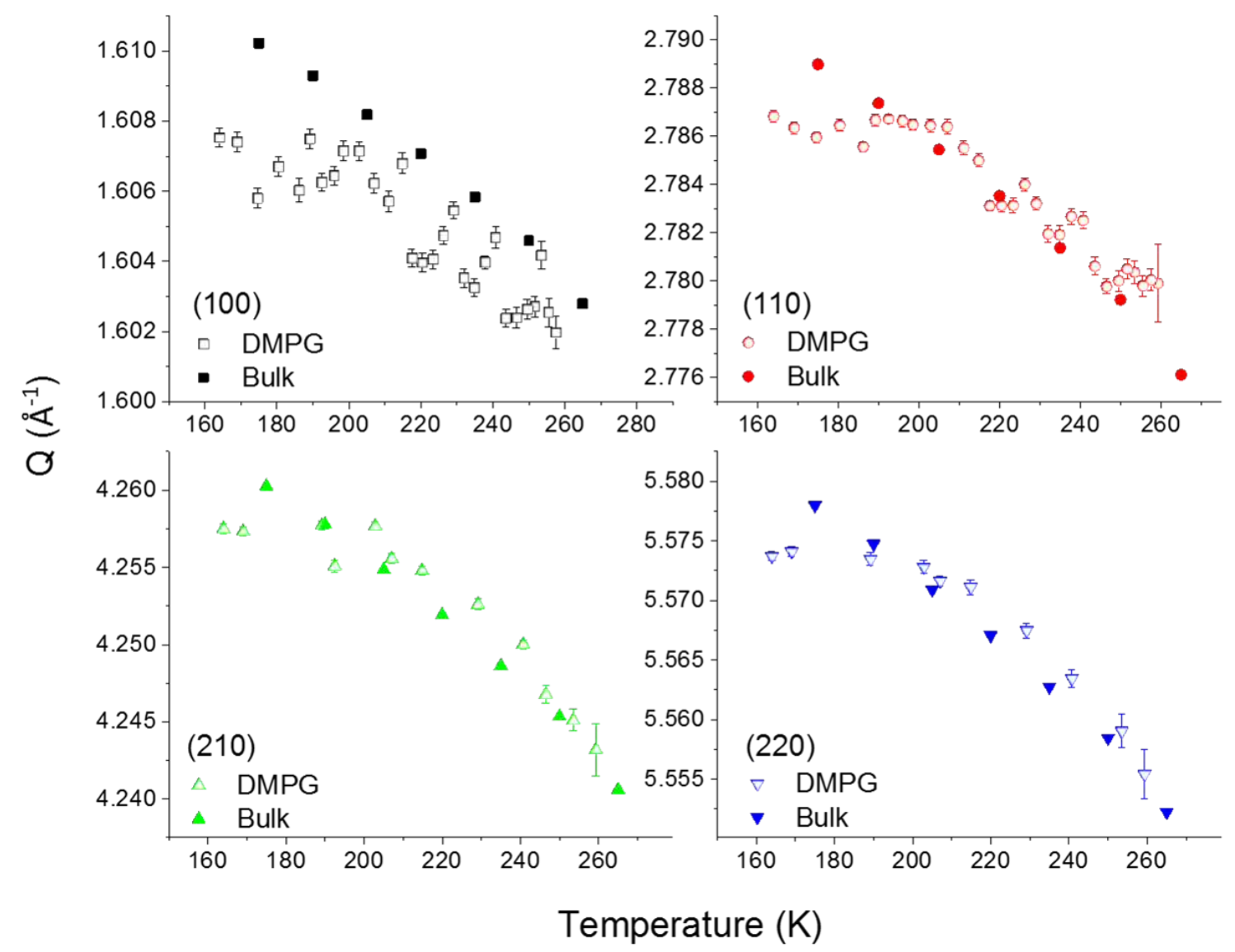

Figure 3.11: Temperature-dependent positions of the four most intense hexagonal $\mathrm{D}_{2} \mathrm{O}$ Bragg peaks measured upon cooling from single-supported DMPG bilayers: (100), (110), (210), and (220). The open data points are the fitted peak positions that have been extracted from neutron diffraction patterns collected on the PSD whereas the colored filled data points indicate peak positions of an isotropic powder of bulk $\mathrm{D}_{2} \mathrm{O}$ determined from Ref. [39]. Hexagonal $\mathrm{D}_{2} \mathrm{O}$ peaks positions measured in DMPG nicely track bulk values until about $200 \mathrm{~K}$ at which point they become temperature-independent. 


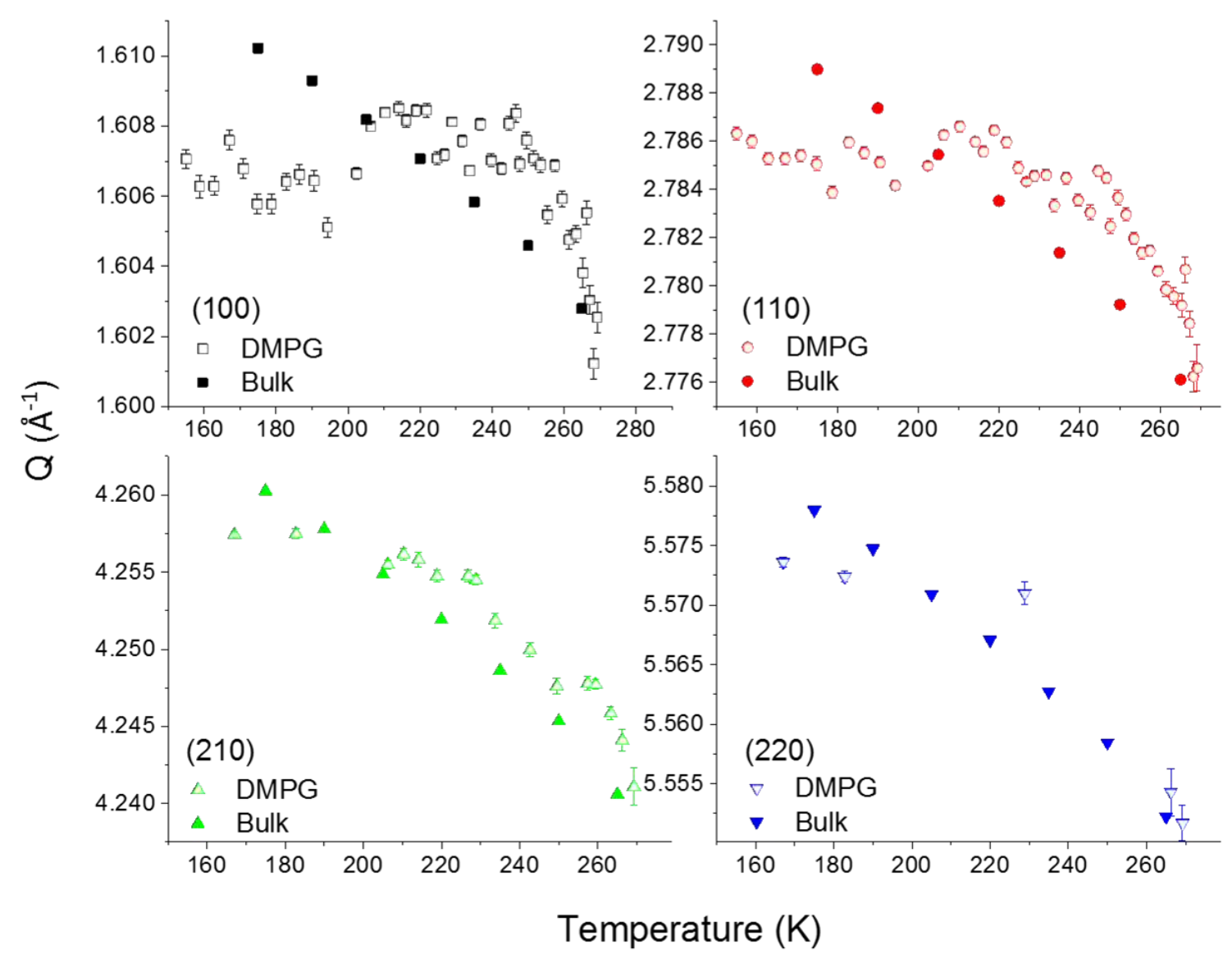

Figure 3.12: Temperature-dependent positions of the four most intense hexagonal $\mathrm{D}_{2} \mathrm{O}$ Bragg peaks measured upon heating from single-supported DMPG bilayers: (100), (110), (210), and (220). The open data points are the fitted peak positions that have been extracted from neutron diffraction patterns collected on the PSD whereas the colored filled data points indicate peak positions of an isotropic powder of bulk $\mathrm{D}_{2} \mathrm{O}$ determined from Ref. [39]. It is noted that the data from Ref. [39] was collected upon cooling. 
positions of the two most intense hexagonal $\mathrm{D}_{2} \mathrm{O}$ Bragg peaks display behavior more similar to bulk.

\subsubsection{Water Structure Associated with Supported Zwitterionic DMPC Membranes}

Just as was the case in the previous section for DMPG, analysis of the Bragg peak intensity as a function of temperature from DMPC membranes hydrated with $\mathrm{D}_{2} \mathrm{O}$ has allowed us to correlate the formation of hexagonal crystalline with the freezing/melting behavior displayed by the hydration water from elastic neutron scans on the HFBS (see Fig. 2.3(a)).

Figure 3.13 shows a full neutron diffraction pattern from DMPC membranes hydrated by $\mathrm{D}_{2} \mathrm{O}$ collected on the PSD at MURR. The pattern in Fig. 3.13 was obtained by subtracting the full pattern collected from $295 \mathrm{~K}$ (room temperature) from the full pattern collected at $255 \mathrm{~K}$. Unlike in the DMPG case, this subtraction reveals only two hexagonal $\mathrm{D}_{2} \mathrm{O}$ Bragg peaks with Miller indices: (100), (110). The two Bragg peaks observed also indicate that the $\mathrm{D}_{2} \mathrm{O}$ hexagonal crystals are oriented with their basal plane parallel to the membrane, similar to what was found for ice associated with DMPG.

Figure 3.14 plots the integrated intensities from the two observed hexagonal $\mathrm{D}_{2} \mathrm{O}$ Bragg peaks, (100) and (110) as a function of temperature. These intensities are compared with those obtained on the HFBS. The same assumption and scaling method was enforced to the DMPC data set as was used for DMPG. We again see that the cooling curves agree, but with a slight temperature shift of approximately $5 \mathrm{~K}$. 


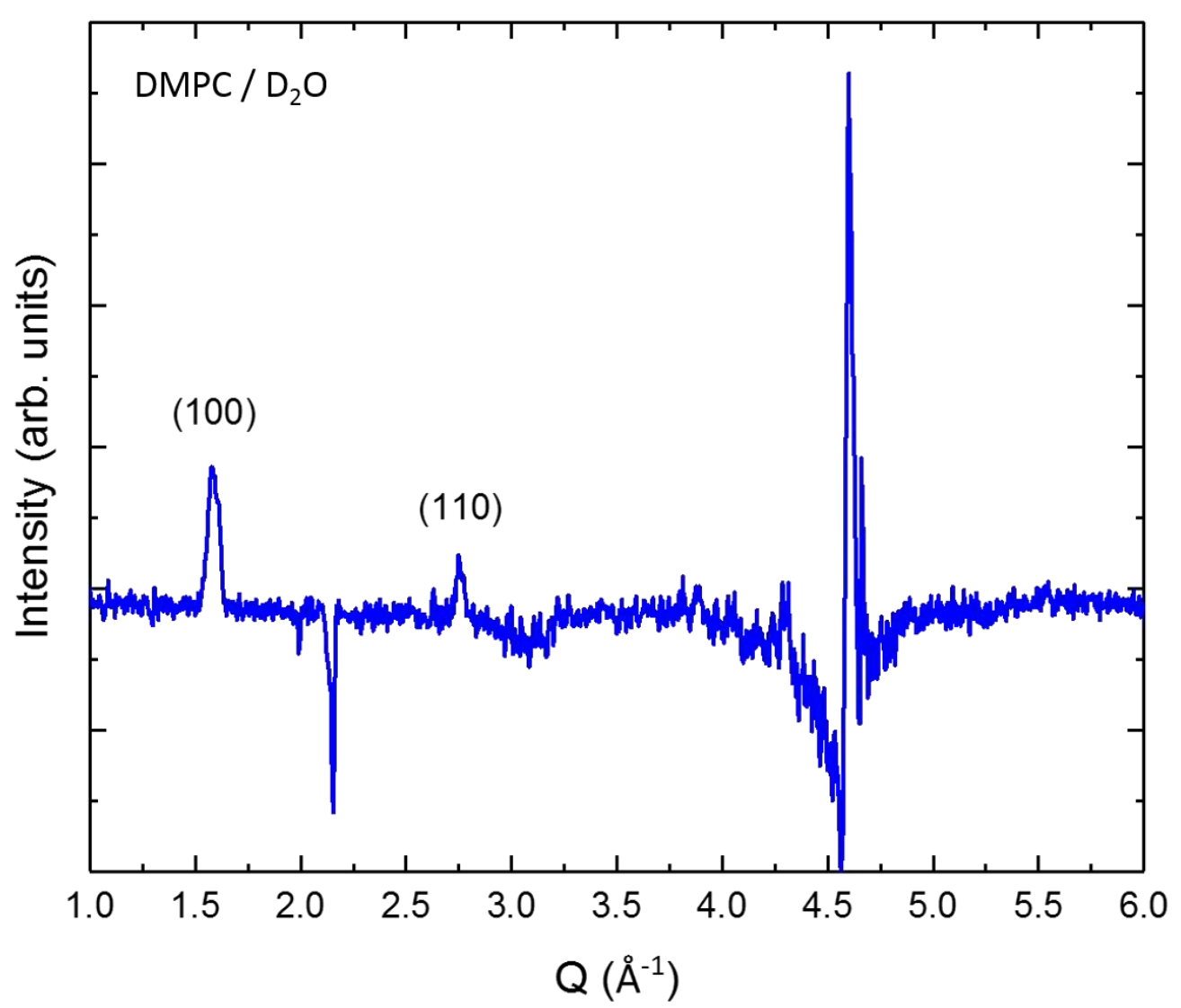

Figure 3.13: Full neutron diffraction pattern of hexagonal $\mathrm{D}_{2} \mathrm{O}$ hydrating DMPC membranes collected at $240 \mathrm{~K}$ measured on the PSD at the University of Missouri Research Reactor. Only two hexagonal $\mathrm{D}_{2} \mathrm{O}$ peaks were observed in the DMPC membrane with their Miller indices indexed in the figure. Data was collected using 4 million neutron monitor counts and summed over all 5 detectors. A scan at $295 \mathrm{~K}$ has been subtracted as background. The sharp spikes in the pattern are a result of detector instabilities during the experiment (see text). 


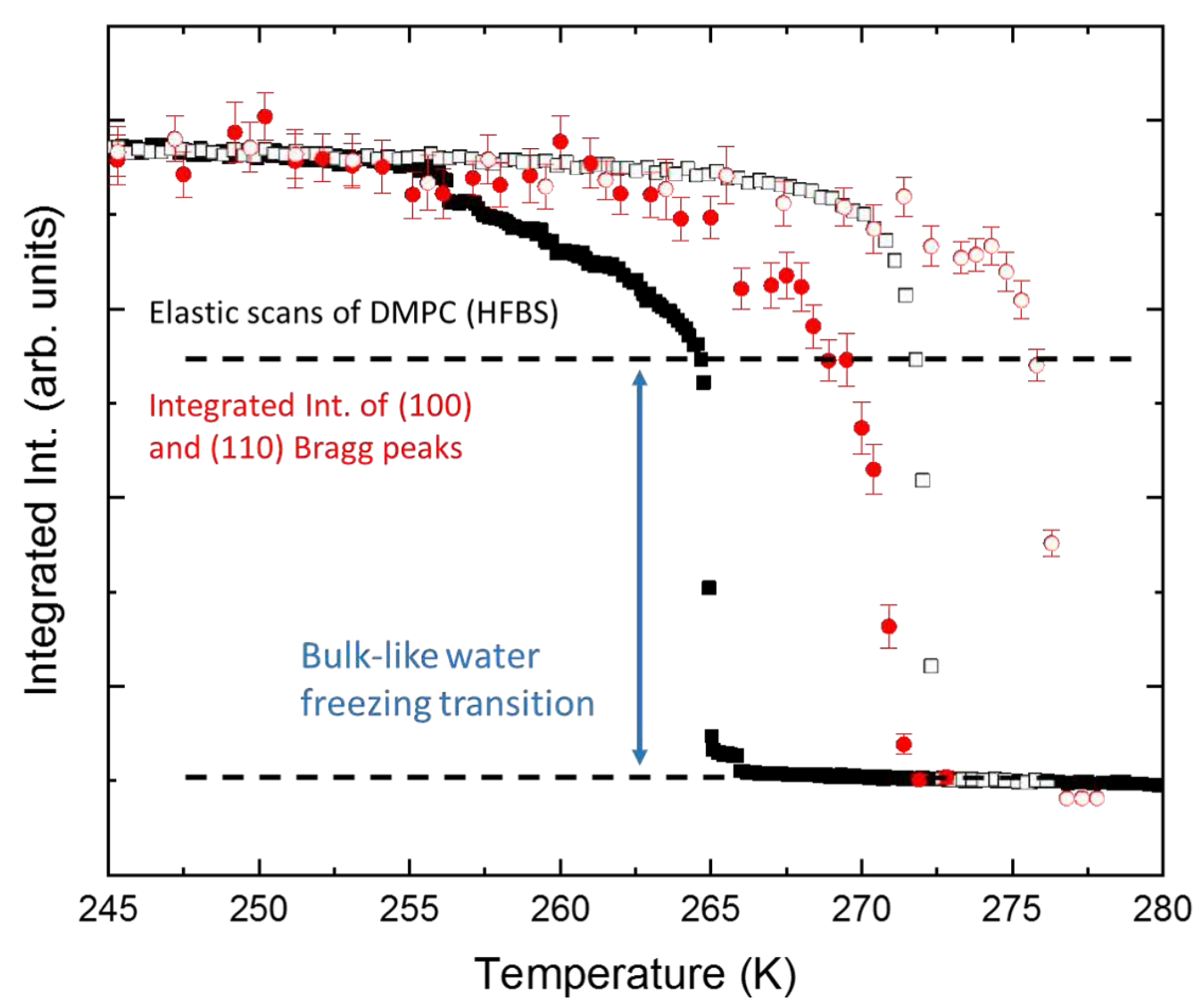

Figure 3.14: Temperature dependence of the elastic neutron intensity obtained from fully-hydrated DMPC bilayers. The incoherent elastic intensity from a membrane hydrated with $\mathrm{H}_{2} \mathrm{O}$ (black squares) was measured on the HFBS whereas the integrated intensity of two Bragg peaks of hexagonal ice were measured on the PSD (red circles) from a membrane hydrated with $\mathrm{D}_{2} \mathrm{O}$. The closed and open symbols represent freezing and heating data, respectively. These measurements on different instruments have been normalized such that the intensities of the initial freezing transition represented by the vertical double arrow are of equal magnitude. The agreement between the two measurements is consistent with all of the water associated with the DMPC membrane freezing into a hexagonal crystalline structure as opposed to an amorphous solid. 


\section{Chapter 4}

\section{Interaction of Water with DMPC Lipid Bilayer Membranes Treated with Melittin Proteins}

In the previous chapters, we used elastic and quasielastic neutron scattering techniques to elucidate the influence that different lipid species have on their membraneassociated water structure, dynamics, and freezing/melting transitions. These experiments laid the groundwork for studying how more complex biological membranes further alter the structure and dynamics of water at various interfaces and confined geometries. In this chapter, we will explore the effects that a model antimicrobial peptide, melittin, has on the structure of single-supported DMPC membranes and their interfacial water dynamics using atomic force microscopy (AFM) and neutron scattering, respectively. 


\subsection{Introduction to Antimicrobial Peptides}

Membrane proteins comprise a diverse class of cellular components paramount for understanding the existence of life and its evolution. Additionally, membrane proteins perform many critical functions that help govern cellular activity and are of ever growing interest in the fields of structural biology, biophysics, and medicine. Perhaps the most studied class of membrane proteins are the antimicrobial peptides (AMP), whose discovery dates back to as early as 1939 [40, 41]. To date, over 5500 natural and synthetic AMPs have been identified [42] some of which possess therapeutic properties that may complement or offer alternatives to existing drugs.

The interaction of AMPs with lipid bilayers and their membrane insertion mechanisms can vary depending on the local water environment surrounding a cell membrane. Unfortunately, the large number of components present in naturally occurring cell membranes (i.e., cholesterol, proteins, and different lipid species) make it difficult to decipher their individual interactions with the hydration water. However, building on previous work $[3,4,43]$, we have fabricated a more biologically relevant model cell membrane by introducing the AMP, melittin, into single-supported DMPC bilayers. The incorporation of melittin into DMPC has allowed us to identify the protein contribution to alterations observed in the membrane-associated water dynamics.

Melittin has been used for decades as a prototype for understanding how more complex AMPs interact with lipid bilayers. When at high enough concentrations, the amphiphilic properties of melittin can result in cell death by inserting, rupturing, and finally destroying the bounding membrane. Cell lysis is common when melittin and similar AMPs encounter a host membrane; however, the insertion mechanisms leading to this outcome require further study. Wimley et al. [44] discuss several models for 
A: Barrel-stave Pore

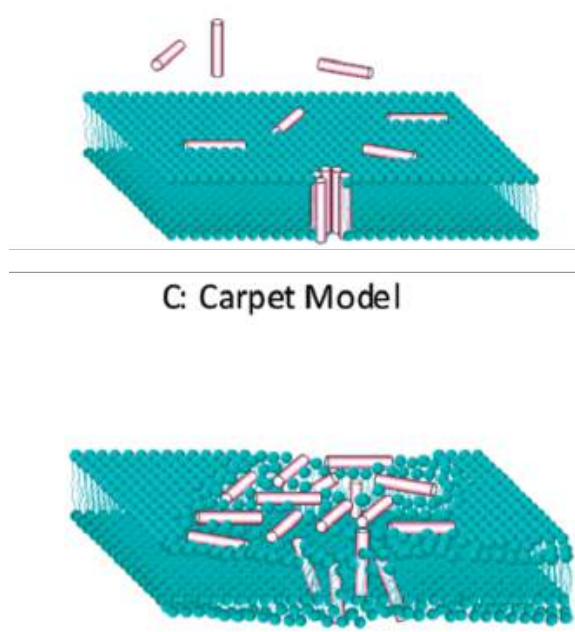

B: Toroidal Pore

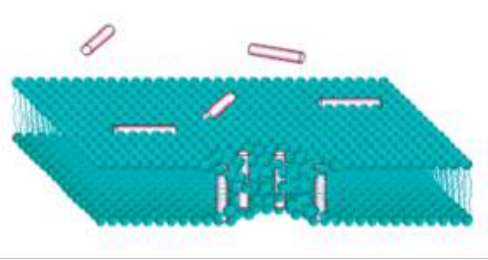

D: Detergent Model

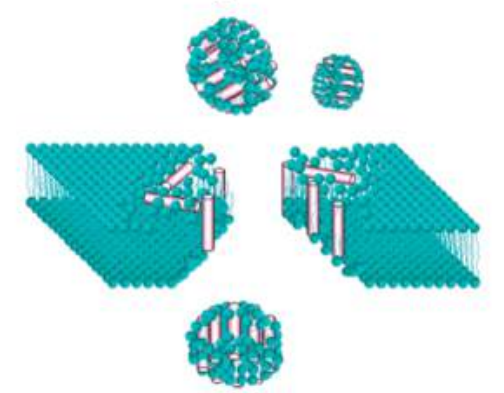

Figure 4.1: Commonly cited models for different antimicrobial peptide insertion. AMP monomers are represented by the red and white linear structures whereas the lipid bilayer is shown in blue. Barrel-stave (a) and toroidal pores (b) are transmembrane structures formed by perpendicularly-oriented peptides, which create aqueous channels that can result in transmembrane leakage. The carpet model (c) depicts aggregates of parallel-oriented peptides embedded in the lipid head group region, which can lead to membrane thinning. At high enough peptide concentrations, saturation of the bilayer can occur, resulting in a structure described by the detergent model (d). Voids in the bilayer are caused by the release of protein-lipid complexes, shown as globular structures in (d). This figure was adopted from Fig. 3 in Ref. [44].

AMP activity and insertion in lipid bilayers.

Figure 4.1 shows multiple pathways for AMP insertion, which can result in pore formation or cell lysis when peptide concentrations exceed a peptide-to-lipid ratio threshold. Results from x-ray diffraction measurements performed on phospholipid multilayers exposed to melittin $[45,46]$ suggest the formation of toroidal pores (see Fig. 4.1(b)); however, depending on its environment, melittin has also been observed to exhibit insertion mechanisms consistent with the carpet model $[47,48]$. We will 
later see in Chapter 5 that AFM measurements on our melittin-treated DMPC membranes reveal bilayer structures similar to those depicted in the carpet and detergent models, Fig. 4.1(c) and Fig. 4.1(d), respectively.

As alluded to earlier, the lytic nature of melittin and similar AMPs makes it possible to tune them for therapeutic applications. Melittin has been used in traditional medicine to treat diseases such as, arthritis and cancer $[49,50]$ and has even displayed inhibition of the human immunodeficiency virus (HIV) [51]. Moreover, the increased resistance by pathogens against traditional antibiotics has sparked a greater interest in the applications of AMPs and their role in advancing novel new medicines and biotechnologies. The potential medical applications, in addition to the variety of insertion mechanisms, drives the need for further understanding the properties of melittin and its interaction with model cell membranes.

\subsection{Properties and Insertion Mechanisms of Melittin Peptides into Lipid Bilayers}

Melittin $\left(\mathrm{C}_{131} \mathrm{H}_{229} \mathrm{~N}_{39} \mathrm{O}_{31}\right)$ is a well-studied antimicrobial peptide (AMP) and the principal toxic component in the venom of the European honey bee, Apis mellifera. It is a small cationic peptide possessing just 26 amino acid residues with sequence, $\mathrm{NH}_{2}$-GIGAVLKVLTTGLPALISWIKRKRQQ-CONH ${ }_{2}$. It has a net charge of +6 , with four of the positively charged residues (KRKR) located in the carboxy-terminal region resulting in a slightly hydrophilic C-terminus whereas the N-terminus is predominately hydrophobic. In its folded state, melittin conforms to a mostly linear structure with residues $2-11$ and $13-26$ adopting $\alpha$-helices [52]. 
Figure 4.2 displays the $\alpha$-helical structure and amphiphilic architecture of a melittin monomer in its folded state. Early measurements by Terwilliger et al. [53] showed by x-ray diffraction that melittin possesses a shape described as a bent rod with the two $\alpha$-helical sections joining at a 'hinge, making an angle of $\sim 120^{\circ}$. This angle can vary depending on the environment and has been measured to be as large as $\sim 160^{\circ}$ when melittin is bound to DMPC bilayers [47]. The length of the peptide possessing an angle of $\sim 160^{\circ}$ is estimated to be about $3.2 \mathrm{~nm}$ [21]. Simplicity of the melittin structure coupled with its amphiphilic nature is characteristic of many other membrane-active proteins, making the melittin peptide a suitable model for studying more complex membrane-protein interactions.

Terwilliger et al. proposed the so-called wedge effect, describing how melittin adsorbs at low concentrations with its principal helical axis parallel to the bilayer surface [53]. Hydrophobic residues oriented mainly toward the inside of the helical bend penetrate the lipid head group region, while hydrophilic residues on the outside of the bend tend to orient away from the membrane center. As melittin concentration increases and aggregates form [19], the area of the outer leaflet of the membrane increases relative to that of the inner leaflet creating tension in the membrane, which can be released by the formation of transmembrane pores lined by the amphiphilic melittin peptide $[45,53]$. Based on their analysis of x-ray diffraction measurements on peptide-lipid multilayers, Lee et al. [45] have argued that the wedge model of Terwilliger et al. [53] applies to transient pores in vesicles through which melittin redistributes to both sides of the membrane prior to forming stable pores.

Melittin monomers remain largely unfolded while free in aqueous solution, but can adopt their amphiphilic and $\alpha$-helical conformation as they approach the interface 


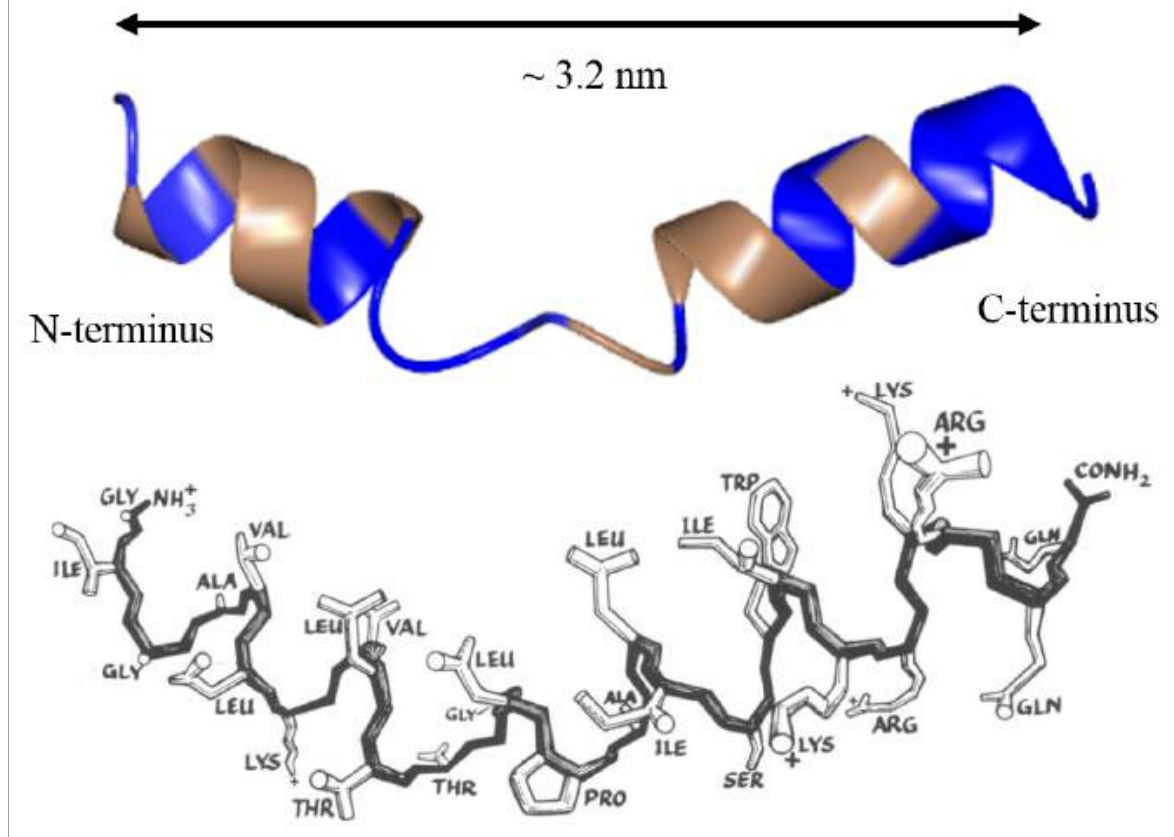

Figure 4.2: Ribbon-like representation (top) and residue side chain (bottom) of a melittin monomer in its folded state. While folded, melittin possesses a mostly $\alpha$ helical conformation with a $\sim 120^{\circ}$ bend at residue position 12 . Hydrophilic and hydrophobic residues in the top figure have been colored blue and gray, respectively. The $\mathrm{N}$ and $\mathrm{C}$ termini are located on the left and right of each figure, respectively. The top image was generated using the CCP4 Molecular Graphics Software [54] based on NMR data retrieved from the Protein Data Bank collected by Perekalin et al. [55] whereas the bottom image was borrowed from Fig. 1 in Ref. [53].

of a lipid bilayer. Membrane-induced secondary structure formation is not unique to melittin and is known to occur for many other small membrane-active peptides. Studies have shown that unfolded melittin peptides undergo conformational changes at the membrane interface due to the more energetically favored hydrogen-bonded peptide bonds compared to their free peptide bonds $[56,57]$. Once folded, the $\alpha$ helical structure and amphiphilic nature of melittin and similar AMPs can result in several possible mechanisms for membrane insertion, inducing surface-bound melittin 
peptides and transmembrane pores [44]. Although the structure of membrane-bound melittin peptides is well-known, their position and orientation within the bilayer is not yet fully understood. Parameters such as humidity, peptide concentration, and temperature have shown to affect the orientation of melittin peptides within a lipid bilayer [58], which may contribute to the various models for peptide insertion (see Fig. 4.1).

We have obtained evidence of a 'carpet-like' model of insertion (see Fig. 4.1) by AFM measurements on melittin-treated single-supported DMPC bilayers. These results are discussed in Chapter 5; however, they are relevant in this chapter as they aid in the interpretation of the neutron scattering data.

\subsection{Elastic Neutron Scattering from Melittin-Treated DMPC Bilayers}

In this section, we discuss results from a series of elastic neutron scattering measurements from water in proximity to membranes of melittin-treated DMPC bilayers. The bare membrane case is similar to that presented earlier in Chapter 2. Elastic scans reveal two distinct freezing transitions of the membrane-associated water, one of which appears dependent upon melittin concentration, suggesting a strong affinity of water to membrane-bound melittin peptides. In addition to temperature dependence measurements, an unexpected time dependence was observed when neutrons were scattered elastically from DMPC bilayers treated within a particular melittin concentration. These measurements suggest the possibility of thermally activated aggregation of melittin peptides within the DMPC bilayer. Aggregation of membrane- 
bound melittin has also been inferred from structural changes observed for a DMPC membrane using AFM, which are believed to be correlated with the newly measured freezing transition. Atomic force microscopy measurements on melittin-treated DMPC membranes will be discussed in Chapter 5 .

We first measured the temperature dependence of neutrons scattered elastically from single-supported lipid bilayers (SSLB) of DMPC treated with various melittin concentrations using the High Flux Backscattering Spectrometer (HFBS). Similarly to the elastic measurements performed on DMPG (see Chapter 2), the signal from samples of melittin-treated DMPC is dominated by a large incoherent scattering from the hydrogen nuclei, most of which belong to water molecules.

\subsubsection{Freezing/Melting Behavior of Water Interacting with Melittin-Treated DMPC Membranes}

Figure 4.3 shows the temperature dependence of elastically-scattered neutrons from water near melittin-treated DMPC membranes upon cooling. Intensities were summed over all 16 detectors of the HFBS, spanning a wave vector transfer range $0.25 \AA^{-1}$ $<Q<1.75 \AA^{-1}$, and normalized to unity at a temperature of $275 \mathrm{~K}$. The elastic scan from the sample made from the solution with the lowest melittin concentration, $0.1 \mu \mathrm{M}$, closely resembles that of the bare DMPC membrane (black squares in Fig. 4.3) except that the bulk-water freezing transition at $265 \mathrm{~K}$ is now slightly broadened. We believe that this broadening results from a dilute dispersion of melittin monomers on the membrane surface, which masks the lipid-water interaction and results in two dominant signals: water interacting with melittin and freezing of bulk-like water. The broadened step in the elastic scan is similar to that which is observed for the freezing 


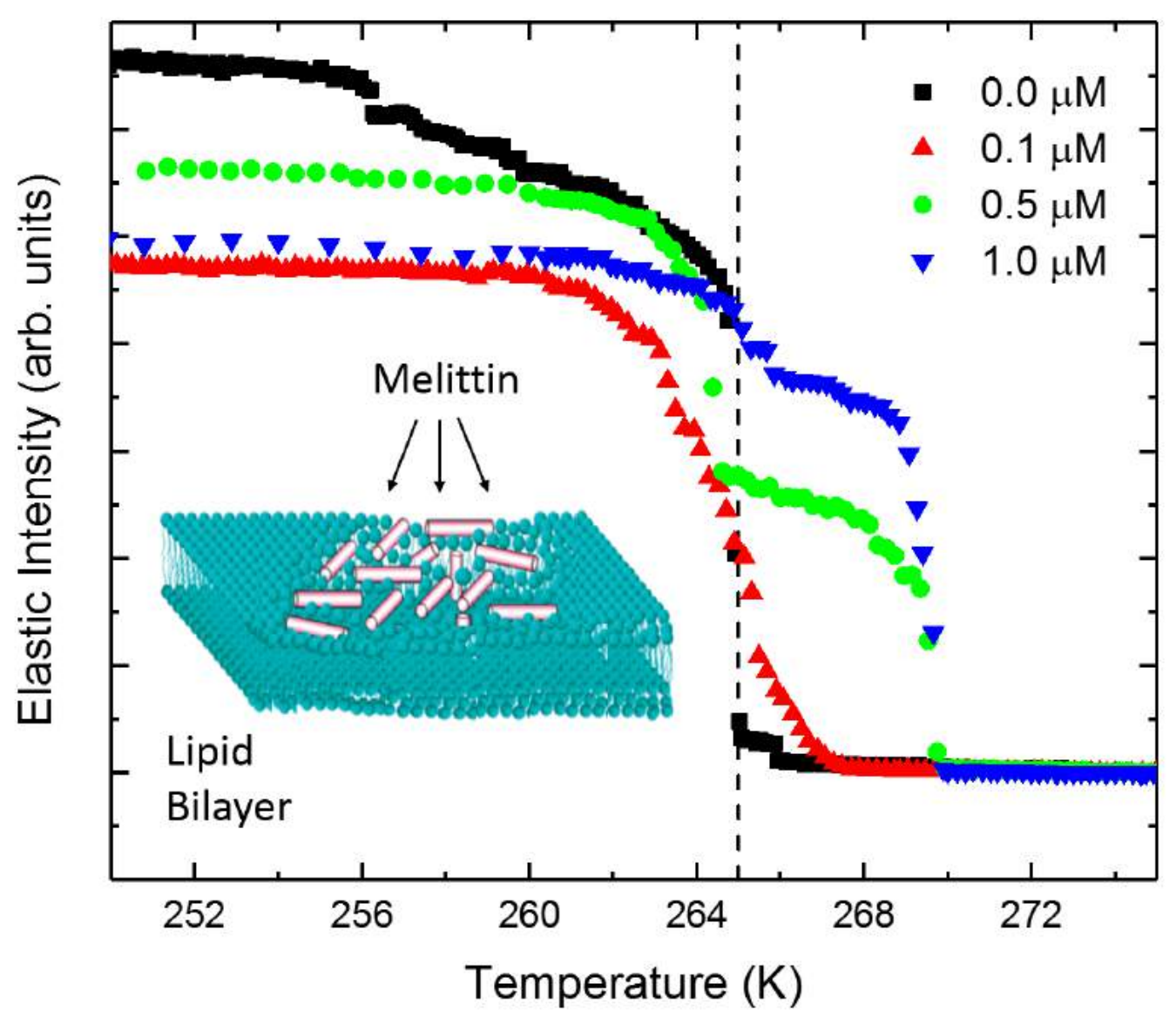

Figure 4.3: Elastically-scattered neutron intensities measured on the HFBS upon cooling from samples of single-supported DMPC bilayers treated with various melittin concentrations. Data from the bare DMPC sample (black squares) were taken from Ref. [4] and reveal an abrupt freezing transition of the bulk-like water at 265 $\mathrm{K}$ followed by a continuous freezing down to $252 \mathrm{~K}$ of confined water which interacts more strongly with the lipid head groups. As the concentration of melittin peptides exposed to the membrane increases, the continuous freezing of water at lower temperatures vanishes and a sharp new freezing transition of the membrane-associated water is observed at $270 \mathrm{~K}$. The change in elastic intensity at the $270 \mathrm{~K}$ freezing step increases in proportion to the melittin concentration, while the height of the step associated with the freezing of bulk-like water, represented by the vertical dashed line, decreases. The inset represents the structure of a DMPC membrane treated with high melittin concentrations and was adopted from Ref. [44]. 
of bulk-like water near a bare DMPG membrane, which is more hydrophilic than DMPC [43]. Note the lower hydration of the $0.1 \mu \mathrm{M}$ sample compared to the bare DMPC sample as indicated by the lower plateau in intensity at low temperature.

As the melittin concentration used to treat the DMPC membranes increases to $0.5 \mu \mathrm{M}$, a sharp freezing transition of the membrane-associated water appears at 270 $\mathrm{K}$ (green circles in Fig. 4.3); and, concomitantly, there is decrease in the height of the freezing step of bulk-like water observed at $265 \mathrm{~K}$ for the bare membrane. Furthermore, on progressing to the $1.0 \mu \mathrm{M}$-treated sample (blue triangles), the size of step in the elastic intensity at $270 \mathrm{~K}$ increases nearly in proportion to the melittin concentration. Upon further cooling, the bulk-like water freezing transition identified in the bare-membrane sample is recovered around $265 \mathrm{~K}$, but with a smaller step upward in intensity. We propose that the newly observed freezing transition at 270 $\mathrm{K}$ results from water freezing onto melittin domains as depicted in Fig. 4.3 and of the type observed in the AFM images (see Chapter 5).

In the case of the bare DMPC membrane, all water melted by $272 \mathrm{~K}$, i.e., below the melting point of bulk ice at $273 \mathrm{~K}$. However, upon heating, the elastic intensity for all three melittin-treated DMPC membranes displays a single sharp melting transition slightly above the bulk-ice melting point that depends on melittin concentration during incubation: $273.3 \mathrm{~K}(0.1 \mu \mathrm{M})$ and $273.7 \mathrm{~K}(0.5$ and $1.0 \mu \mathrm{M})$. This melting behavior provides further evidence that water is interacting strongly with the membrane-bound melittin.

Figure 4.4 shows the data of three consecutive cooling and heating cycles measured on the HFBS from the $0.5 \mu \mathrm{M}$-melittin sample. In all cases, the freezing and melting behavior of the membrane-associated water was found to be reproducible over many 


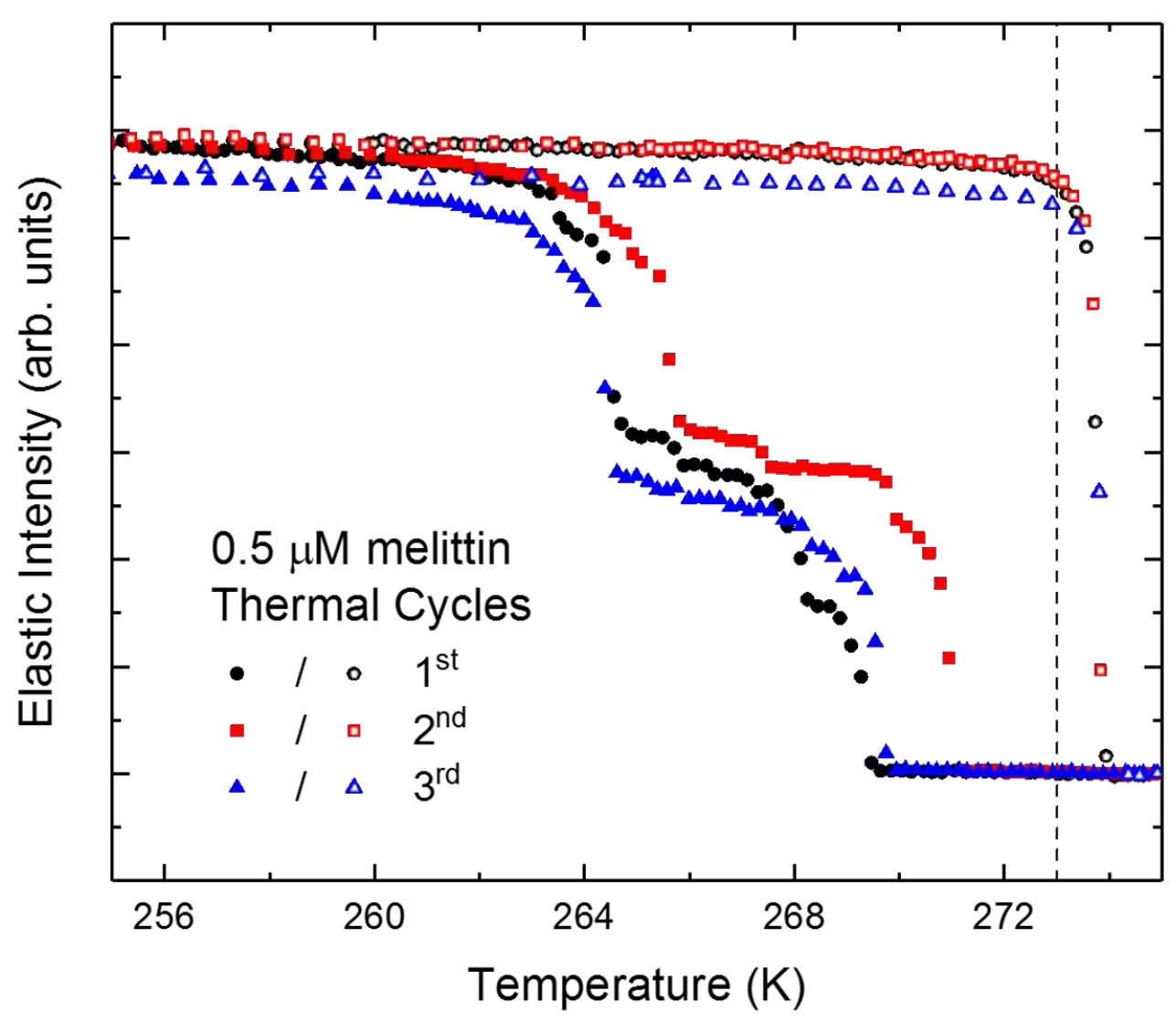

Figure 4.4: Consecutive elastic neutron scans as a function of temperature measured on the HFBS from single-supported DMPC bilayers treated with a solution containing melittin peptides at a concentration of $0.5 \mu \mathrm{M}$. The sample was subjected to a 5 -hour anneal at $328 \mathrm{~K}$ before each cycle except for the second scan (red points) before which the sample was annealed for 2 hours. The strong agreement between thermal cycles indicates the sample was of high-quality. The data has been normalized such that the intensities of the cooling curves at $275 \mathrm{~K}$ are unity, with each data point representing 5 minutes of collection time. The vertical dashed line represents the temperature at which bulk-water freezes. All the membrane-associated ice abruptly melts just above the bulk melting point, in contrast to the bare membrane case for which melting occurred at the bulk value. 
thermal cycles. Upon heating DMPC bilayers treated with $0.5 \mu \mathrm{M}$ melittin (open symbols in Fig. 4.4) we observe a complete melting of the membrane-associated water above the bulk melting point of water. Upon cooling (closed symbols in Fig. 4.4), two distinct freezing transitions of roughly equal changes in intensity can be seen; however, the temperatures at which they occur can vary by a couple of degrees. The slight shift in temperature of these freezing transitions is believed to be a result of different anneal times. All membranes were subjected to an anneal at $328 \mathrm{~K}$ in between thermal cycles for up to 5 hours. During the annealing process, an unexpected increase in the elastic intensity was observed for DMPC membranes treated with $0.5 \mu \mathrm{M}$ melittin. Interestingly, this effect was absent for the other DMPC membrane samples exposed to $0.1 \mu \mathrm{M}$ and $1.0 \mu \mathrm{M}$.

\subsubsection{Annealing of Melittin-Treated DMPC Bilayers}

In the previous section, we described the temperature dependence of the elasticallyscattered neutron intensity from water hydrating melittin-treated DMPC membranes and demonstrated its reproducibility upon consecutive thermal cycles (see Fig. 4.4). However, before a full thermal cycle was performed, the melittin-treated DMPC membranes were annealed at $328 \mathrm{~K}$ for up to 5 hours. The anneal temperatures for our melittin-treated samples were determined from AFM measurements of the temperature dependence of the DMPC bilayer thickness that showed a transition into its fluid phase at about $328 \mathrm{~K}$ while in air (see Chapter 5). Previous measurements on bare membranes have shown that annealing is critical for maintaining membrane homogeneity between thermal cycles. The greater annealing temperature forces the membrane into a more fluid state, increasing the likelihood of lipid molecules being 
able to fuse and repair defects that may have occurred as a result of water freezing onto the membrane surface.

For a narrow range of peptide concentrations, an interesting and unexpected time dependence of the elastic intensity was observed during an anneal at $328 \mathrm{~K}$. Elastic neutron scattering intensities from DMPC membranes exposed to an intermediate melittin concentration of $0.5 \mu \mathrm{M}$ steadily increased for the duration of the anneal, whereas elastic intensities obtained from $0.1 \mu \mathrm{M}$ and $1.0 \mu \mathrm{M}$ melittin-treated DMPC remained constant.

Figure 4.5 shows the time-dependent elastic neutron intensities during annealing of the three melittin-treated DMPC membranes used in gathering data shown in Fig. 4.3: (a) $0.1 \mu \mathrm{M}$, (b) $0.5 \mu \mathrm{M}$ and (c) $1.0 \mu \mathrm{M}$. Plotted on the vertical axis are the raw intensities, which were obtained by summing over all wave vector transfers $Q$ and normalizing to the HFBS monitor counts. All samples were subjected to a minimum 3-hour anneal, which was found to be a sufficient time for melittinlipid interactions to achieve equilibrium within supported bilayers [21]. The increase in elastic intensity over time suggests that hydrogen nuclei are slowing down on a timescale of the instrumental resolution $(\sim 4 \mathrm{~ns})$; however, only the $0.5 \mu \mathrm{M}$-melittin sample showed a significant increase. The slopes for the linear fits displayed in Fig. $4.5(\mathrm{a}),(\mathrm{b})$, and $(\mathrm{c})$ are $2.48 \times 10^{-8}, 14.2 \times 10^{-8}$, and $0.935 \times 10^{-8}$, respectively (arb. units/second).

During the anneal at $328 \mathrm{~K}$, the DMPC lipids are in their fluid phase, and therefore possess a greater mobility than in their gel phase. The combination of temperature, appropriate melittin concentration, and the high lipid mobility is believed to facilitate the aggregation of surface-bound melittin, a hypothesis supported by AFM measure- 


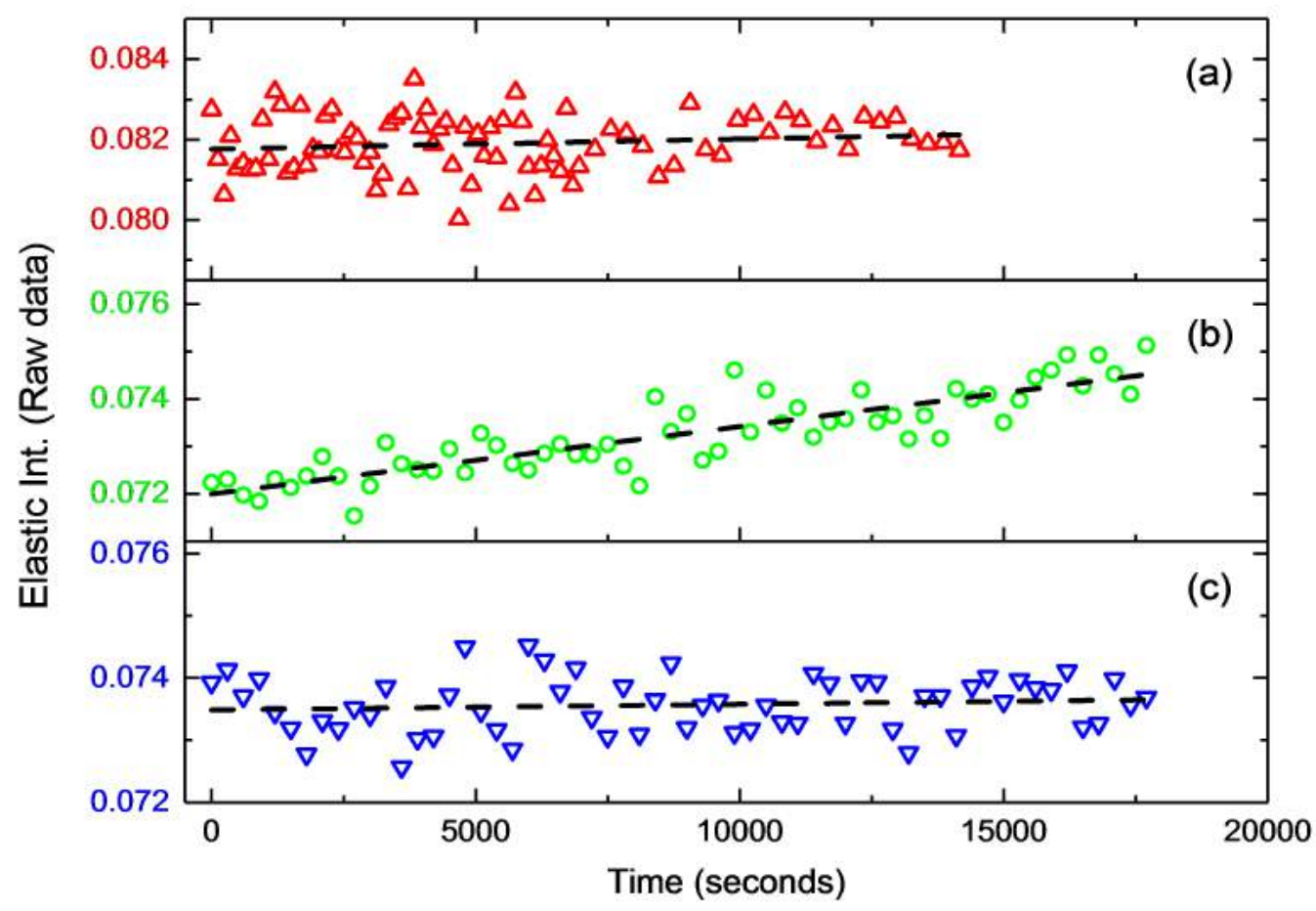

Figure 4.5: Elastic neutron scattering intensity as a function of time for DMPC membranes treated with (a) $0.1 \mu \mathrm{M}$, (b) $0.5 \mu \mathrm{M}$ and (c) $1.0 \mu \mathrm{M}$ melittin collected on the HFBS during an anneal at $328 \mathrm{~K}$. The dashed black lines in each panel are best linear fits to the data. For a melittin concentration of $0.5 \mu \mathrm{M}$, the elastic intensity steadily grows for the duration of the anneal, suggesting an increase in the nummber of hydrogen nuclei moving on a timescale longer than 4 ns. This increase is unique for intermediate peptide concentrations and does not occur in the other two melittin concentrations (including the bare membrane). 
ments that show the formation of dimple-like features on the surfaces of melittintreated DMPC bilayers after they have been annealed (see Chapter 5). The elastic intensity of SSLB DMPC membranes treated with melittin concentrations of $0.5 \mu \mathrm{M}$ increase by as much as $4.7 \%$ during annealing at $328 \mathrm{~K}$ over a period of several hours. This behavior is consistent with the diffusion and subsequent anchoring of melittin into domains, such as those depicted in Fig. 4.1. Estimates of a peptide-to-lipid ratio from AFM measurements suggest the contribution from hydrogen in the melittin is negligible, implying that the subtle increase in elastic intensity during annealing is possibly due to hydrogen atoms in the lipids having their motion reduced by the wedge effect of the melittin.

These initial anneal scans motivated further investigation into the effects melittin concentration, anneal duration, and temperature have on the elastic neutron intensity and membrane morphology. In Chapter 5 we explore the effects of morphological changes due to annealing melittin-treated DMPC membranes using atomic force microscopy, but for now we will continue to discuss additional measurements of the time-dependent elastic intensity performed on the HFBS to elucidate membranebound peptide aggregation.

Figure 4.6 shows annealing elastic neutron scans collected at $328 \mathrm{~K}$ from two different samples of DMPC, both treated with $0.5 \mu \mathrm{M}$ melittin. The red data points in Fig. 4.6 are from the original $0.5 \mu \mathrm{M}$ sample measured in December 2014, which appears in Fig. 4.3 and Fig. 4.5(b). However, the blue data points in Fig. 4.6 come from a sample that was fabricated in May 2015 using similar melittin peptides at the same $0.5 \mu \mathrm{M}$ concentration. The May 2015 sample was annealed at $328 \mathrm{~K}$ for 8 hours and compared to earlier annealing data. As expected, there was again a very similar 


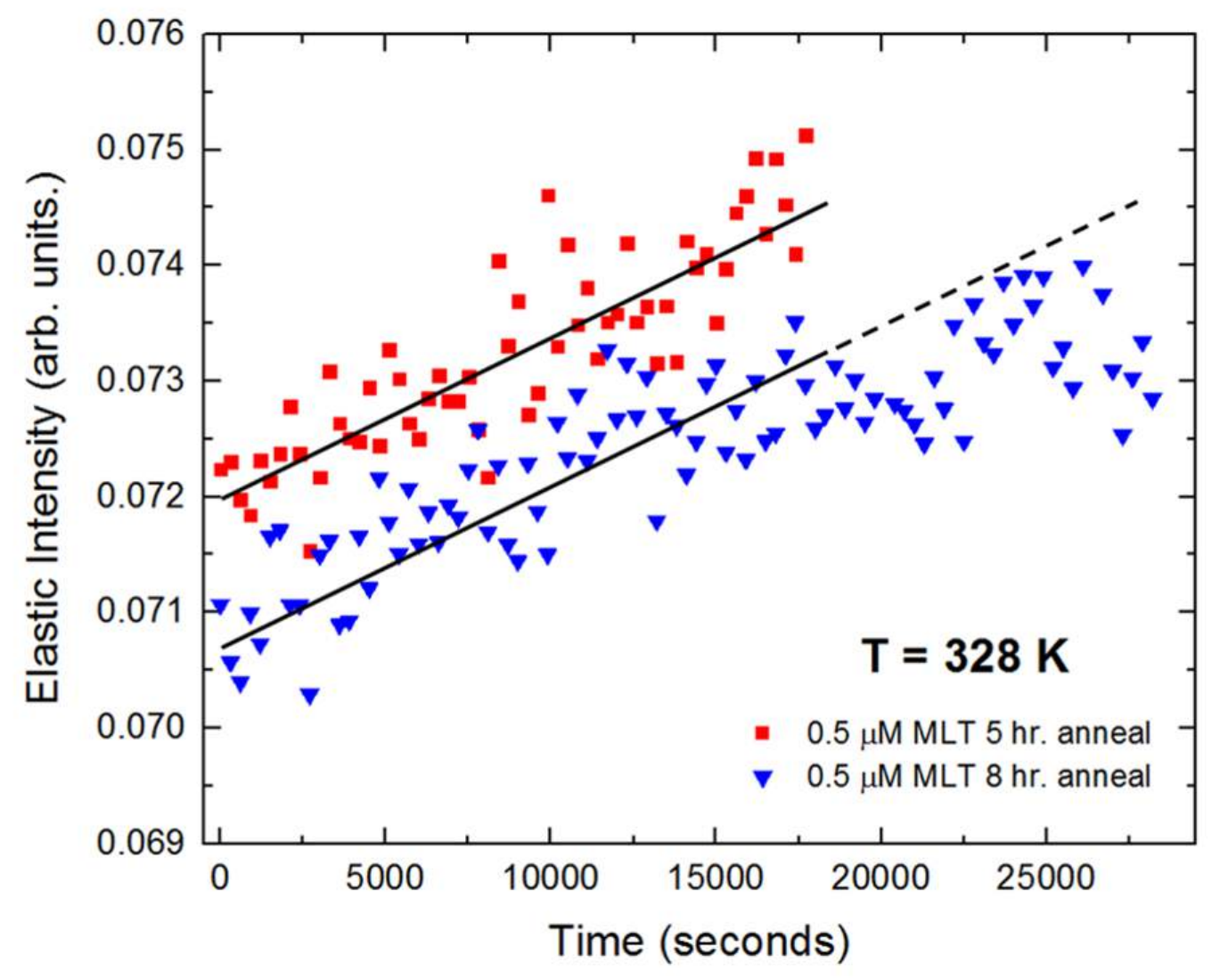

Figure 4.6: Time-dependent elastic neutron scattering raw intensities collected on the HFBS at $328 \mathrm{~K}$ from two different DMPC membranes treated with $0.5 \mu \mathrm{M}$ melittin. The red data points comes from a sample measured in December 2014 and are the same data set appearing in Fig. 5.2.3(b). The blue data points were collected from a sample fabricated in May 2015 and measured for 8 hours, during which time a similar increase in the elastic intensity was observed. The solid black lines represent the constant rise in intensity similar to the two samples. The dashed line indicates the region where elastic intensity from the May 2015 sample starts to level off. 
increase of the elastic intensity as a function of time. Interestingly, once anneal times exceeded 5 hours, the elastic intensity began to level off as indicated by the dash line in Fig. 4.6. This behavior is intuitively pleasing because there exist only so many hydrogen nuclei that could be participating in this motion and suggests all of the membrane bound peptides have aggregated into large clusters and are now moving on a time scale longer than the instrumental resolution. Further evidence of this has been revealed via atomic force microscopy measurements and will be discussed in Chapter 5 .

Attempts to comprehensively measure this annealing effect at various temperatures in order to extract an activation energy for peptide aggregation was conducted in January 2016. Results from these annealing measurements can be seen in Fig. 4.7.

Figure 4.7 shows a series of 5 anneal scans measured on the HFBS at different temperatures from a freshly made sample of DMPC treated with $0.5 \mu \mathrm{M}$ melittin. An initial temperature-dependent elastic scan was collected from this sample (see Fig. B.1), but did not show two well-defined freezing transitions of equal intensity change as was originally observed (see. Fig 4.3). However, there was a sharp freezing step at $270 \mathrm{~K}$, which had been previously identified as water freezing as a result of melittin and therefore the annealing measurements were carried out on this sample.

For temperatures greater than $328 \mathrm{~K}$ we observe a steady increase in the elastic intensity as a function of anneal time for DMPC membranes treated with $0.5 \mu \mathrm{M}$ melittin (Fig. 4.7). The data in Fig. 4.7 were fitted with a linear function in order to determine total change in intensities. The results from fitting the annealing data in Fig. 4.7 are shown in Table 4.1. Also Tabulated in 4.1 are the order in which these temperatures were measured. The low and high intensity values in Table 4.1 
Table 4.1: Summary of Annealing Scans for $0.5 \mu \mathrm{M}$ melittin-treated DMPC

\begin{tabular}{|c|c|c|c|c|}
\hline Temp (K) & Order & Low Int. (arb. units) & High Int. (arb. units) & \% Change \\
\hline 320 & 2 & 0.08931 & 0.08925 & -0.06723 \\
\hline 325 & 4 & 0.08919 & 0.08922 & 0.03362 \\
\hline 328 & 5 & 0.08915 & 0.08876 & -0.4394 \\
\hline 330 & 1 & 0.08693 & 0.08863 & 1.918 \\
\hline 340 & 3 & 0.08703 & 0.08781 & 0.8883 \\
\hline
\end{tabular}

were determined from the linear fit to the elastic intensity as a function of time for each of the 5 anneal runs. The fact that intensity increases are only observed at temperatures greater than $328 \mathrm{~K}$ suggest there is a temperature threshold for which melittin peptides begin the aggregation process. 


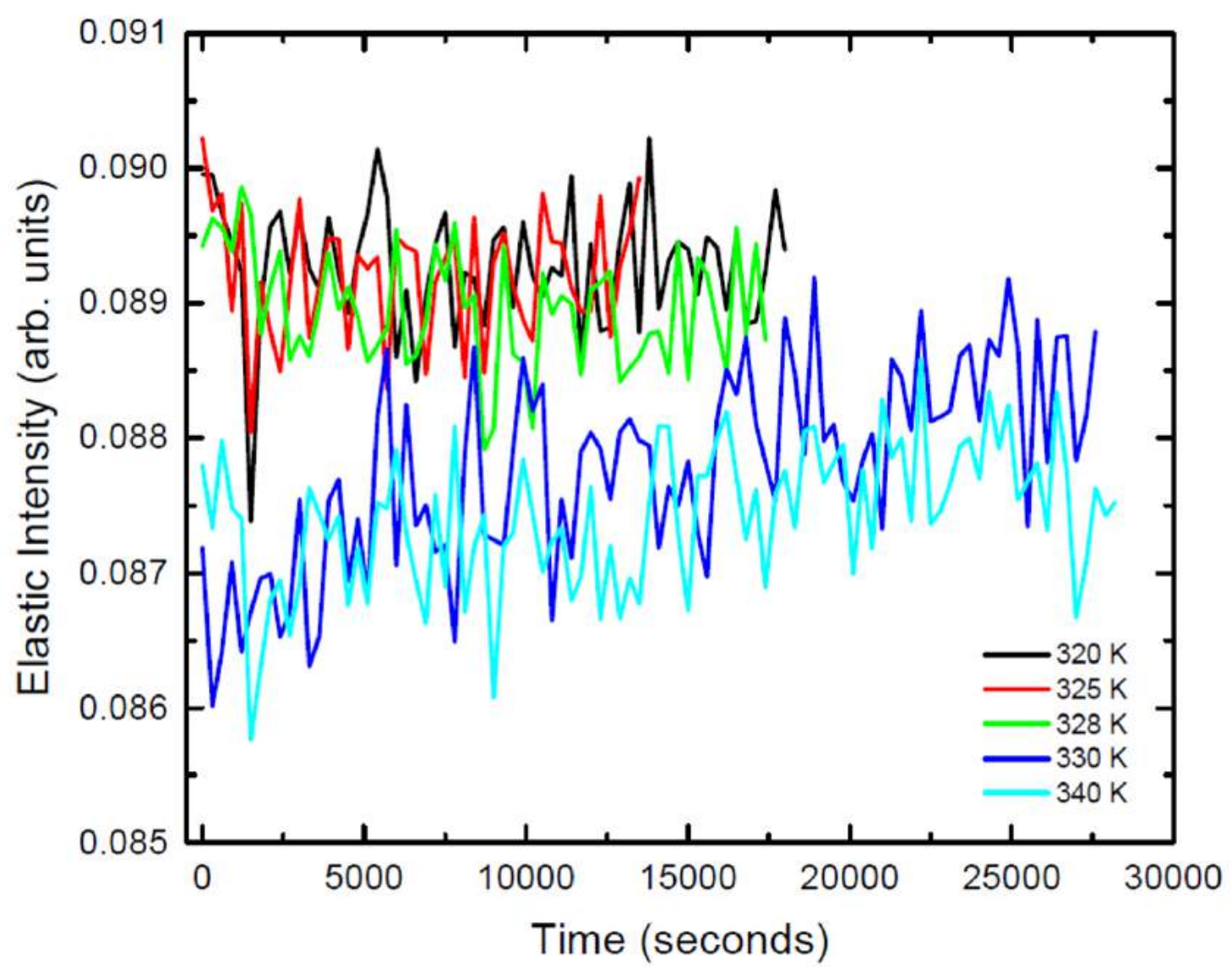

Figure 4.7: Time-dependent elastic neutron scattering intensities collected on the HFBS at various temperatures from DMPC membranes treated with $0.5 \mu \mathrm{M}$ melittin. All 5 scans were collected from the same sample, which was fabricated in January 2016. The order at which data was collected at different temperatures is shown in Table 4.1. Each data set was fitted with a linear function (not shown) in order to extract slopes that allowed a \% change in intensity to be calculated. 


\subsection{Inelastic Neutron Scattering from Melittin-Treated DMPC Membranes}

To understand how the presence of melittin in DMPC bilayers affects the vibrational modes of interfacial water molecules, inelastic neutron scattering measurements were performed on the Fine-Resolution Fermi Chopper Spectrometer (SEQUOIA) at the Spallation Neutron Source (SNS) at Oak Ridge National Lab (ORNL). SEQUOIA is a direct geometry (incident neutron energy is fixed) time-of-flight spectrometer with incident neutron energies in the range $8 \mathrm{meV}<\mathrm{E}_{i}<2000 \mathrm{meV}$ and with an energy resolution typically on the order of $1-3 \%$ of $\mathrm{E}_{i}$, allowing various dynamical properties of materials to be investigated including magnetic excitations and lattice vibrations.

Analysis of QENS spectra from hydrated melittin-treated DMPC membranes suggests the existence of a water component, which undergoes a slower lateral diffusion compared to bulk water. It is therefore hypothesized that water participating in this motion may possess a vibrational density of states that differs from bulk-like water. Shifts in the O-H stretching mode of crystallized water have been observed before in confined systems using inelastic neutron scattering [59], making this technique desirable for elucidating the interfacial water dynamics in supported membrane systems. Here, we will discuss results obtained from SEQUOIA on SSLBs and use them to help interpret the dynamics observed from QENS measurements.

The sample environment used on SEQUOIA possesses a carousel-style mounting plate where three samples can be secured simultaneously and lowered into the scattering chamber, providing an advantage for quick data collection times without having to break vacuum, warm up, and change samples. Once inelastic spectra from a sample 

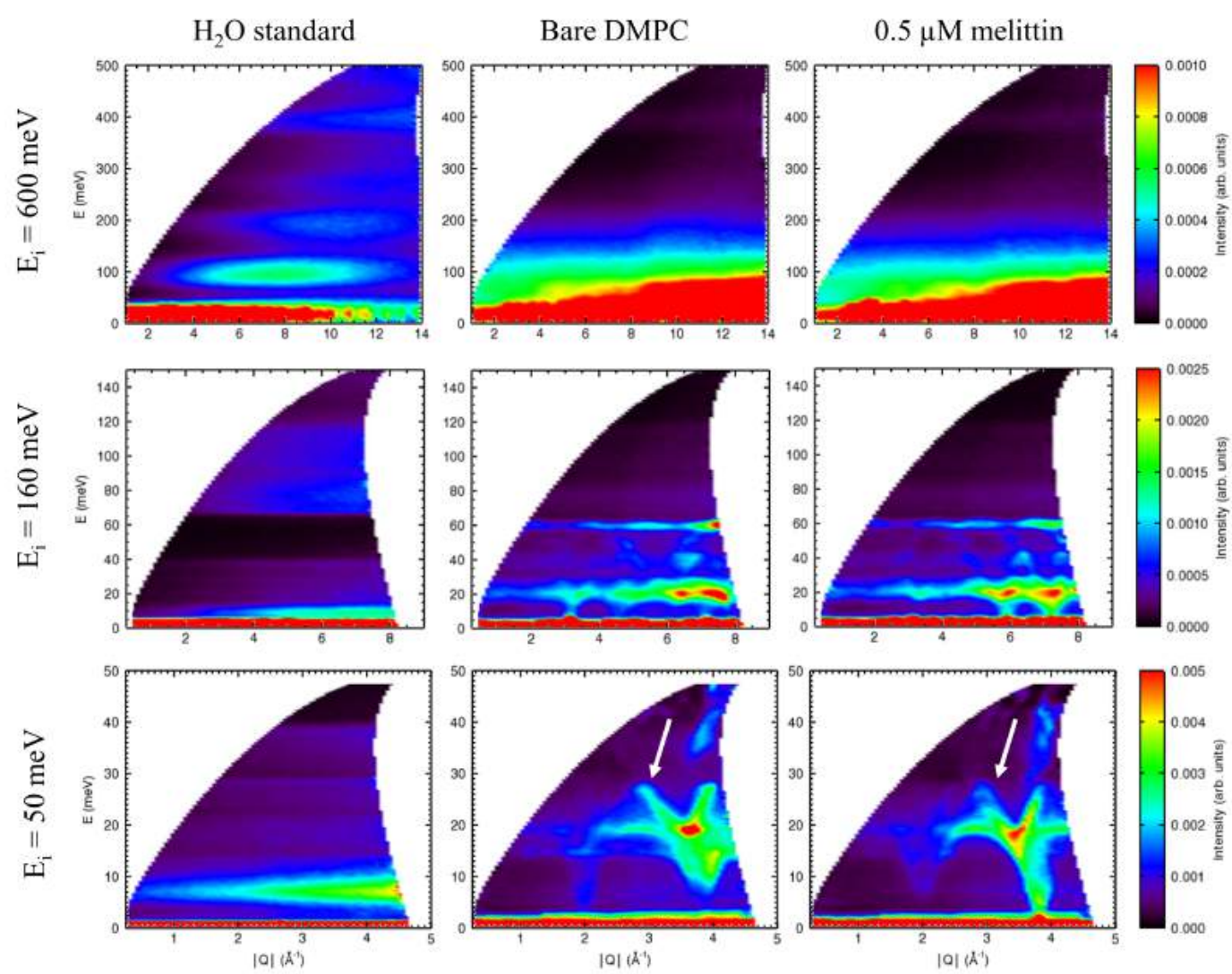

Figure 4.8: Inelastic neutron scattering color plots collected from bulk water (left column), bare DMPC (middle column) and $0.5 \mu \mathrm{M}$ melittin-treated (right column) membranes measured on SEQUOIA. The top, middle, and bottom panels represent the data sets obtained from incident neutron energies of $600 \mathrm{meV}, 160 \mathrm{meV}$, and $50 \mathrm{meV}$, respectively. Each matrix element in the above figure plots the scattered neutron intensity (color scale) as a function of neutron energy transfer and wave vector transfer $Q$. These color maps aid in interpreting what regions of $\omega$ and $Q$ space are viable for observing various vibrational modes of water. For low incident energies, parabolic-like features (indicated by white arrows) in the intensities can be seen for both membrane samples. These represent the phonon spectra of Si. Data were collected at $5 \mathrm{~K}$ for 60 minutes. 
have been collected, the carousel rotates a different sample into the incident beam. Repeating this process for various incident energies allows us to compare several data sets spanning $Q-\omega$ space to obtain the density of states for the vibrational modes of interest. Here we are interested in the $\mathrm{O}-\mathrm{H}$ stretching mode of water, which is known to be excited in ice at energy transfers of $400 \mathrm{meV}$. The three samples chosen for this measurements were a pure bulk water standard contained in a annular cell, a bare DMPC sample with low hydration (as determined from previous BASIS measurements), and a $0.5 \mu \mathrm{M}$ melittin-treated DMPC membrane (the same one measured on BASIS). The incident neutron beam on SEQUOIA was aligned parallel to the wafer surfaces.

Once secured under vacuum in the sample environment, the three sealed samples were cooled quickly from room temperature to $5 \mathrm{~K}$. The incident energies chosen to probe the vibrational modes of water were $600 \mathrm{meV}, 150 \mathrm{meV}$, and $60 \mathrm{meV}$ with the bulk water standard being the first measured. The optimal slit dimensions for ensuring maximum sample exposure while avoiding unwanted scattering from the samples' aluminum flange were determined to be $50 \mathrm{~mm}$ wide and $35 \mathrm{~mm}$ high. Knowing the annular bulk water cell has inner and our radii of $7.85 \mathrm{~mm}$ and 7.95 $\mathrm{mm}$, respectively, one can estimate the total water volume exposed to the beam to be about $0.173 \mathrm{~cm}^{3}$. Therefore, the total amount of water contributing to the standard spectra is estimated to be roughly $5.38 \times 10^{21} \mathrm{H}_{2} \mathrm{O}$ molecules (assuming the density of water at $5 \mathrm{~K}$ to be $\left.0.93 \mathrm{~g} / \mathrm{cm}^{3}\right)$.

Figure 4.8 contains the resulting color intensity maps obtained from the three samples for each of the incident energies. They show the scattered neutron intensity (color scale) as a function of wave vector transfer $Q$ and and energy transfer $\omega$. The 


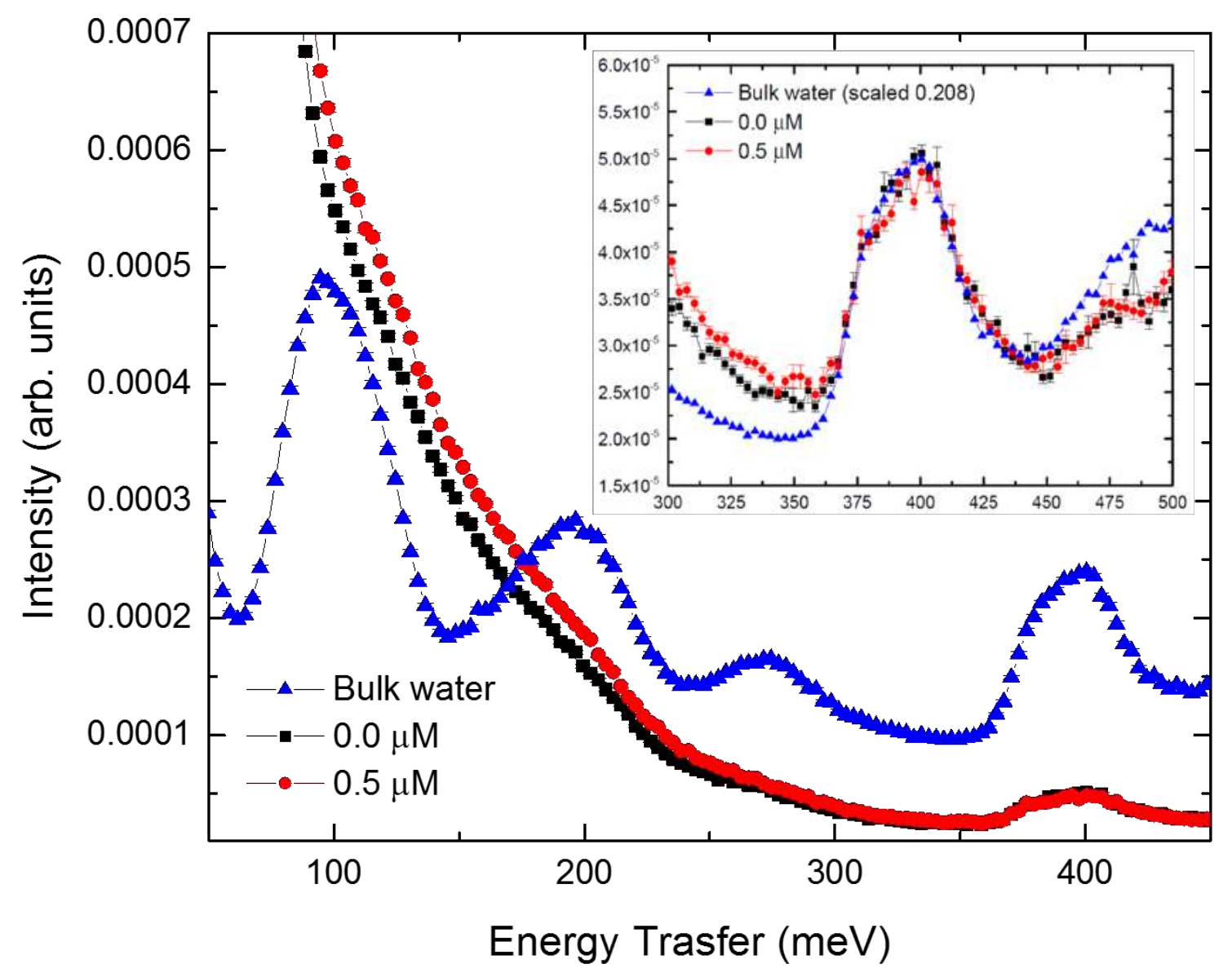

Figure 4.9: Vibrational density of states of water interacting with single-supported melittin-treated DMPC membranes averaged over wave vector transfers, $6 \AA^{-1}<Q$ $<12 \AA^{-1}$. Data from the bulk water standard, bare DMPC, and melittin-treated DMPC are shown in blue, black, and red, respectively. The inset is a blow-up region of the region of the $\mathrm{O}-\mathrm{H}$ stretching mode with the bulk water data being scaled by a factor of 0.208 for clarity. There is no obvious shift in the $\mathrm{O}-\mathrm{H}$ stretching for water interacting with the membrane samples, suggesting most of the signal is bulk-like. 
red area near zero energy transfer represents the elastic scattering of the sample; but, at larger energy transfers, the vibrational modes of water begin to emerge.

Figure 4.9 shows the vibrational density of states of the three samples: bulk water standard sample (blue curve), bare DMPC (black curve), and $0.5 \mu \mathrm{M}$ melittin (red curve). Fig. 4.9 was obtained by 'slicing' a region of $Q$ space from the plots shown in the first upper row of Fig. $4.8\left(\mathrm{E}_{i}=600 \mathrm{meV}\right)$. Peaks located at $100 \mathrm{meV}$, $200 \mathrm{meV}$, and $400 \mathrm{meV}$ in the bulk water data represent librational, bending, and stretching modes, of the $\mathrm{O}-\mathrm{H}$ bond respectively. The $\mathrm{O}-\mathrm{H}$ stretching mode at 400 $\mathrm{meV}$ is also observed in our membrane samples, but is roughly a factor of 5 weaker in intensity (see inset in Fig 4.9). Based on the known amount of water in the standard $\mathrm{H}_{2} \mathrm{O}$ sample, we estimate roughly $1.12 \times 10^{21}$ water molecules participate in the $\mathrm{O}-\mathrm{H}$ stretching mode in the bare and melittin-treated DMPC membranes. Assuming the bulk density of water, this number of water molecules is equivalent to a uniform film of water of thickness $\sim 83 \mathrm{~nm}$ on each side of 100 wafers.

The data in Fig. 4.9 does not show any clear shift in the O-H stretching mode when comparing bare DMPC and DMPC membranes treated with $0.5 \mu \mathrm{M}$ melittin. With an estimated $83 \mathrm{~nm}$ thick water slab hydrating the membranes, it is possible that the signal from bulk water drowns out any dampening effects on the molecular vibrations caused by the presence of lipids and melittin. 


\subsection{Quasielastic Neutron Scattering from Melittin- Treated DMPC Bilayers}

The $0.5-\mu \mathrm{M}$ melittin sample was chosen for further investigation by QENS based on the elastic scans showing two distinct freezing transitions of the membrane-associated water at $270 \mathrm{~K}$ and $265 \mathrm{~K}$ that had comparable changes in elastic intensity. Therefore, full quasielastic spectra were collected on BASIS from $0.5 \mu \mathrm{M}$ melittin-treated DMPC bilayers over the temperature range where the two freezing transitions were observed.

\subsubsection{Comparison of Quasielastic and Elastic Intensities from Melittin-Treated DMPC Membranes}

On cooling of the $0.5 \mu \mathrm{M}$ melittin-treated sample, we obtained QENS spectra with counting times of 1 hour at a temperature interval of $0.5 \mathrm{~K}$ over a temperature range that included the freezing transitions. The spectra were fitted using the DAVE software [26] by folding the instrumental resolution function with a scattering law composed of three terms: a delta function corresponding to the elastic scattering and two Lorentzian terms representing the quasielastic scattering. The QENS spectra of the $0.5 \mu \mathrm{M}$ melittin sample was analyzed similarly to DMPG (see Chapter 2) in order to isolate two distinct diffusive processes occurring on different time scales: a "fast" motion described by the broad Lorentzian and a "slow" motion represented by the narrow Lorentzian in addition to the elastic component.

Figure 4.10 shows the temperature-dependent intensities from all three spectra components obtained by fitting the $0.5 \mu \mathrm{M}$-melittin sample. In panel (a), we see that the elastic component is in qualitative agreement with that measured with a different 


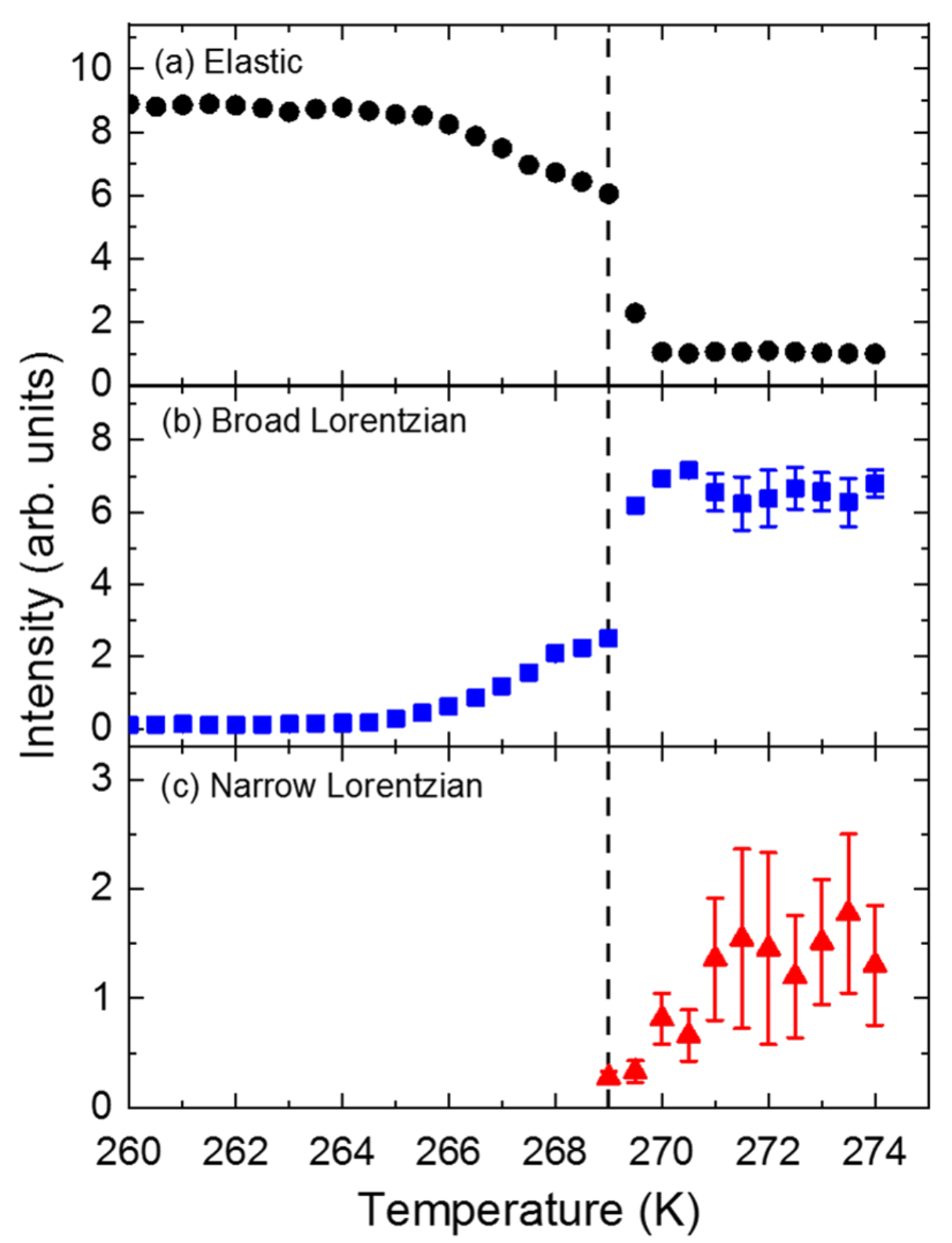

Figure 4.10: Temperature-dependence of the intensity of the three components in the QENS spectra from $0.5 \mu \mathrm{M}$ melittin-treated DMPC measured on BASIS upon cooling. (a) The elastic intensity has been summed over all $Q$ and normalized to unity at $275 \mathrm{~K}$; (b) integrated intensity of the broad-Lorentzian component representing fast motion; and (c) integrated intensity of the narrow-Lorentzian component representing slow motion. Both quasielastic terms have been summed over the $Q$ values used to obtain the diffusion coefficients, $0.5 \AA^{-1}, 0.7 \AA^{-1}$, and $0.9 \AA^{-1}$ and normalized to the elastic intensity at $275 \mathrm{~K}$. The vertical black dashed line at $269 \mathrm{~K}$ represents the temperature at which the transition attributed to the freezing of bulk-like water onto the peptides completes. 
sample on the HFBS (cf. Fig. 4.3, green circles). On cooling, there is again an abrupt upward step in the elastic intensity at $\sim 269 \mathrm{~K}$, which we have attributed to bulk-like water freezing onto the peptide. It is followed by a wider step near $267 \mathrm{~K}$, which is somewhat weaker and broader than the one at $265 \mathrm{~K}$ in the HFBS elastic scan. We attribute these differences to a greater heterogeneity in the BASIS sample, its somewhat lower purity (85\%), and possibly differences in thermometry and method of temperature control. The elastic scans on the HFBS were collected continuously with their intensities averaged over 5 minutes with a cooling rate of $0.04 \mathrm{~K} / \mathrm{min}$ whereas the BASIS data were obtained at a fixed temperature for 1 hour.

Figure 4.10(b) shows that a step-like decrease in the intensity of the broad Lorentzian occurs at the same temperature as the step-like increase in the elastic component (Fig. 4.10(a)) that was attributed to the freezing of bulk-like water onto the peptide. At lower temperature, there is a plateau in intensity followed by a more gradual decrease in the broad Lorentzian intensity, matching the wider step upward in the elastic intensity near $267 \mathrm{~K}$. Based on this correspondence between the intensities of the elastic and broad-Lorentzian components in the QENS spectra, we identify the broad Lorentzian as representing the diffusion of bulk-like water. Its temperature dependence below $269 \mathrm{~K}$ may result from the freezing of bulk-like water over regions of the DMPC membrane unoccupied by melittin.

\subsubsection{Diffusion of Water Hydrating Melittin-Treated DMPC Membranes}

The half-width-at-half-maximum (HWHM) $\Gamma$ of both Lorentzian components in the

BASIS spectra exhibits a $Q^{2}$ dependence at low $Q$, characteristic of translational 
diffusion. Performing a least squares fit to Ficks Law $\left(\Gamma=D Q^{2}\right)$ at low $Q$, we obtained a diffusion coefficient $D$ for both the broad- and the narrow-Lorentzian components in the spectra. The weaker intensity of the narrow Lorentzian made fitting the QENS spectra with two components difficult. Therefore, in order to resolve the narrow component and infer a diffusion coefficient from it, we began our fitting procedure by fixing the width of the broad Lorentzian at values corresponding to those of bulk supercooled water at temperatures above $269 \mathrm{~K}$. This constraint allowed a stable two-Lorentzian fit to the spectra initially, and subsequently it could be relaxed. We were unable to fit spectra collected below $269 \mathrm{~K}$ reliably using two Lorentzians.

Figure 4.11 contains the diffusion coefficient as a function of temperature determined for bare DMPC and for the two Lorentzian components in the spectra of the melittin-treated DMPC. At temperatures above $268 \mathrm{~K}$, the diffusion coefficient obtained from the broad-Lorentzian component in the spectra of the melittin-treated sample (blue square points) tracks that identified with bulk-like water in the bare DMPC sample as well as that of bulk supercooled water to within error. This behavior is consistent with the identification of the broad-Lorentzian component with bulk-like water made from the analysis of its intensity in Fig. 4.10. However, below $268 \mathrm{~K}, D$ begins to deviate from the bulk behavior, decreasing steadily before leveling off at $264 \mathrm{~K}$, the same temperature at which the elastic intensity plateaus (see inset to Fig. 4.11). We have previously identified the plateau in $D$ of the bare DMPC membrane in the temperature range $262 \mathrm{~K}<T<265 \mathrm{~K}$ with a water type termed "confined 1", which remains mobile below the freezing transition of bulk-like water and which may be located in the region between bulk ice and the lipid head groups [43]. The discrepancy with the diffusion coefficient inferred from the broad 


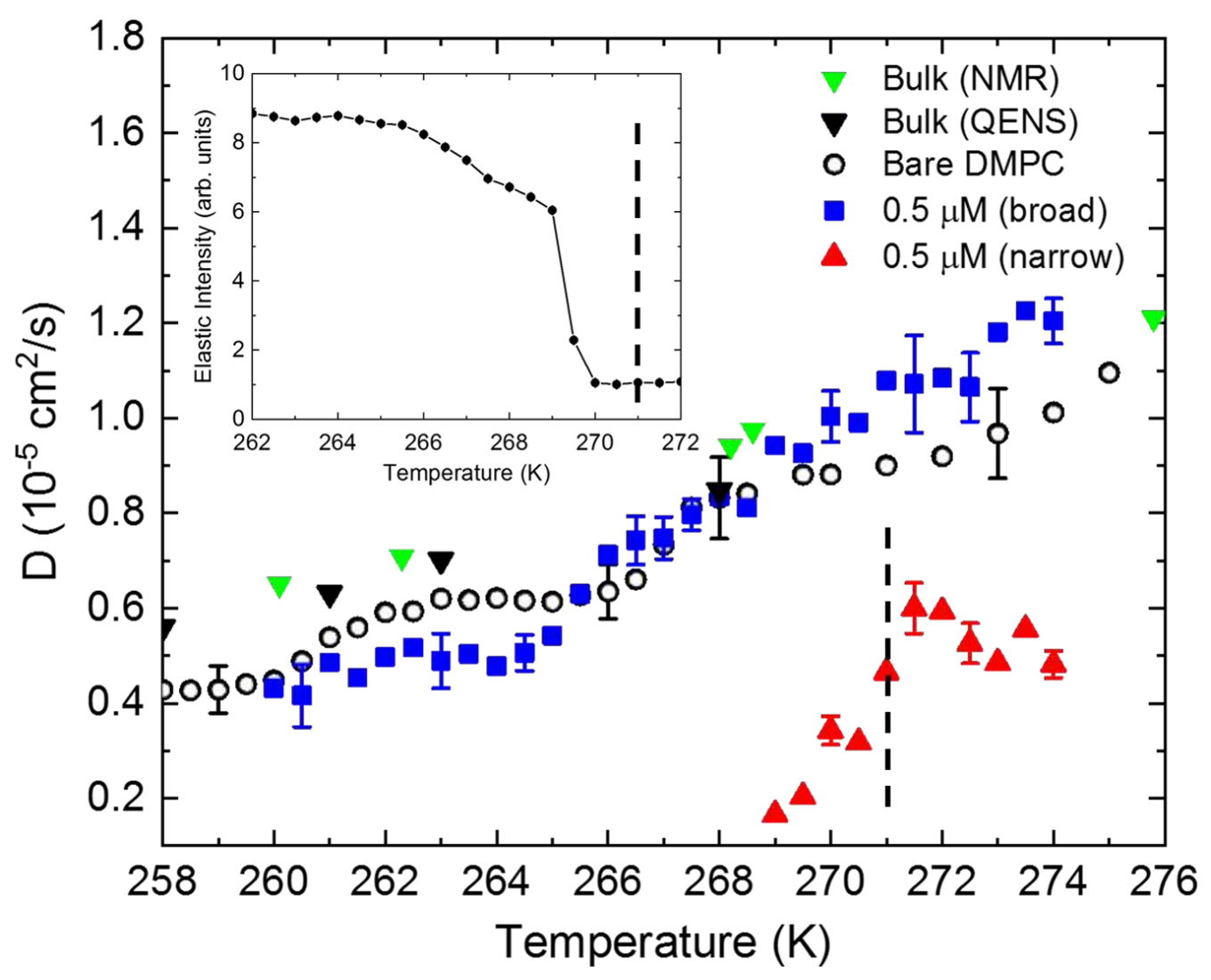

Figure 4.11: Temperature dependence on cooling of the two diffusion coefficients inferred from analysis of the QENS spectra from DMPC membranes treated with 0.5 $\mu \mathrm{M}$ melittin: broad Lorentzian (blue squares) and narrow Lorentzian (red triangles). For comparison, the values of $D$ from bare DMPC membranes (open black squares) from Ref. [4] and bulk-supercooled water (green triangles) from Ref. [31] are also shown. The inset contains the elastic intensity of the melittin-treated sample obtained on BASIS (from Fig. 4.10(a)). The dashed black vertical line at $271 \mathrm{~K}$ indicates the temperature at which the diffusion coefficient associated with the narrow component begins to decrease. 
Lorentzian of the melittin-treated sample in this temperature range suggests that the confined 1 water also freezes onto the peptide.

The diffusion coefficient inferred from the narrow-Lorentzian component (red triangles in Fig. 4.11) has a magnitude roughly half that of the bulk-like component at $273 \mathrm{~K}$ and displays a qualitatively different temperature dependence, which tracks that of the narrow-Lorentzian intensity in Fig. 4.10(c). Above $271 \mathrm{~K}, D$ is constant to within the measured uncertainty, but at lower temperatures decreases to a value $0.22 \times 10^{-5} \mathrm{~cm}^{2} / \mathrm{s}$ at $\sim 269 \mathrm{~K}$ where the intensity of the narrow component vanishes. We suggest that this more slowly diffusing water population is located closer to the melittin aggregates and interacts more strongly with them than the bulk-like water.

\subsection{Conclusion}

We have investigated the diffusion $D$ of water in proximity to a DMPC membrane on which melittin peptides have been deposited. These concentrations of melittin have shown to strongly effect the freezing behavior of the membrane-associated water by introducing a newly observed freezing transition at $270 \mathrm{~K}$, in addition to the freezing of bulk-like water at $265 \mathrm{~K}$ (cf. Fig. 4.3). The presence of two well-defined freezing transitions suggests the existence of two types of water diffusing on different time scales. Upon analyzing QENS spectra from DMPC membranes treated with 0.5 $\mu \mathrm{M}$ melittin, we find evidence of two populations of water indicated by broad and narrow Lorentzians, representing fast and slow motion, respectively. Both Lorentzian components exhibit a $Q$ dependence of their half-widths-at-half-maxima indicative of translational diffusion. The broad Lorentzian yielded diffusion coefficients similar to bulk water for $268 \mathrm{~K}<T<274 \mathrm{~K}$ whereas analysis of the narrow Lorentzian yielded $D$ 
values that were roughly half those of bulk water at the same temperature. Below 269 $\mathrm{K}$, the narrow Lorentzian vanishes from the spectra, indicating a stronger interaction with the peptide than the bulk-like water. We speculate that larger proteins may exhibit more than the two freezing transitions that we have found for water onto melittin and thereby have to protein reveal the relative strength of different binding sites for water. Also, a more slowly diffusing water component in proximity to the inserted peptide may be a feature common to other non-pore-forming antimicrobial peptides.

Our results suggest a role for QENS measurements on SSLBs in complementing other techniques such as, the ODNP-enhanced Nuclear Magnetic Resonance (NMR) for investigation of water dynamics near membrane-bound proteins. In addition to the need to determine the effect of the spin-label on the system of interest, the NMR technique is limited to room-temperature measurements. By performing QENS measurements at lower temperatures, it is possible to freeze out different types of motion such as that of bulk water. Although a QENS measurement involves an average over a macroscopic sample, the wave vector $Q$ and energy dependence $\omega$ of the QENS spectra allow one to separate molecular motions from distinct water populations characterized by their respective length and time scales. Therefore, by combining AFM (see Chapter 5) with QENS measurements on single-supported membrane samples of sufficient homogeneity, one is able to probe both the structure of the bound peptides and the dynamics of the membrane-associated water on the nanoscale. 


\section{Chapter 5}

\section{Microscopy of Melittin-Treated DMPC Lipid Bilayers}

In this chapter, we will discuss the effects of melittin peptides on membrane structure in single-supported lipid bilayers of DMPC. Melittin $\left(\mathrm{C}_{131} \mathrm{H}_{229} \mathrm{~N}_{39} \mathrm{O}_{31}\right)$, the principal toxic component in bee venom, is a well-studied antimicrobial cationic peptide known to insert into both zwitterionic and anionic membranes [60], making it an ideal candidate for study. Its amino acid sequence and amphiphilic properties allow it to adopt various confirmations while interacting with lipid membranes. Adjusting parameters such as temperature, peptide concentration, or hydration can cause melittin monomers to insert parallel or perpendicular with respect to the membrane plane [58], resulting in several possible pathways for subsequent peptide aggregation and/or pore formation. To help elucidate such behavior and insertion mechanisms of melittin in our model cell membranes, AFM measurements were performed on DMPC bilayers in the presence of melittin as a function of peptide concentration and temperature. 


\subsection{Overview of Atomic Force Microscopy}

Atomic Force Microscopy (AFM) is a spatially precise high-resolution technique used to study the topography of nanoscale materials for a wide range of applications in physics, semiconductor technology, and molecular biology. The length scales achievable with AFM are commensurate with the size of molecules such as small proteins, phospholipids and deoxyribonucleic acid (DNA) [61]. Lateral and vertical resolution can reach as low as $10 \mathrm{~nm}$ and $100 \mathrm{pm}$, respectively, making AFM a valuable tool for investigating the structure and interactions of biological systems. Furthermore, AFM measurements can be performed under conditions emulating the natural environments of the biological species of interest, allowing for controlled in-situ imaging of, for example, proteins inserting into a lipid membrane.

With a similar operating principle as a profilometer, AFM aims to map out the sample surface using a sharp tip on the end of a flexible cantilever, which can be engineered to possess an apex radius on the order of $8 \mathrm{~nm}$. When an AFM tip is brought in close proximity to a material of interest, it will interact with its surface via electrostatic and van der Waals forces causing the cantilever to deflect. These deflections are primarily caused by the tip's interaction with a topological feature on the sample and are measured by reflecting a laser from the back of the cantilever into a photodiode as the tip scans across the surface.

In addition to three-dimensional imaging, AFM is also useful for investigating the mechanical properties of materials. Instead of scanning an area of the sample, one fixes the $x$ and $y$ positions, while carefully approaching the tip to the sample surface and monitoring the cantilever deflection as a function of $z$-distance. This

procedure is known as a force approach method and, as the names suggests, can 
provide information regarding the forces at play near the tip/sample interface as well as elastic properties of the material being interrogated. Force approach measurements can also be used to determine forces required for proteins to undergo folding and insertion into supported lipid membranes [62], making the AFM an ideal instrument for studying protein/membrane interactions.

Topographical images from melittin-treated DMPC bilayers fabricated for neutron scattering measurements were collected under ambient conditions using a Digital Instruments Nanoscope IIIa AFM at the University of Missouri. In order to visualize structural changes to the membrane induced by melittin in real time, in situ imaging was also performed on similarly prepared bilayers supported on mica substrates using a Cypher ES Environmental AFM at the Center for Nanophase Material Sciences at Oak Ridge National Laboratory. In both cases, AFM images were recorded under tapping mode using silicon nitride tips (radius ; $10 \mathrm{~nm}$ ) possessing spring constants and resonant frequencies in the range of $0.10 .3 \mathrm{~N} / \mathrm{m}$ and $20-40 \mathrm{kHz}$, respectively. Neither AFM set-up had sufficient lateral resolution to allow imaging of individual melittin monomers.

\subsection{Effects of Temperature on Melittin-Treated DMPC Bilayers}

Fluidity is an important property of cell membranes as it helps facilitate the insertion and subsequent aggregation of various proteins. Lipid molecules that comprise a cell membrane experience a range of dynamics, from lateral diffusion to rotation and libration, resulting in the membrane behaving as a two-dimensional fluid. The degree 
of fluidity depends on factors such as temperature, the presence of cholesterol, and whether the lipid tails possess saturated or unsaturated fatty acids. If the lipid species is known, one indicator for determining the phase of its membrane is bilayer thickness.

In the fluid state, the 14 carbon atom long tails of a DMPC lipid are collapsed into a more globular configuration, lowering the energy barrier proteins need to overcome in order to insert into the bilayer. However, when temperatures fall below the gel-to-fluid phase transition, the bilayer thickness increases due to the extension and stiffening of the lipid tails. As a result, the lipids become tightly packed and their tails more rigid, making it difficult for proteins to penetrate the head groups in order to begin their insertion process. For temperatures above the gel-to-fluid transition, DMPC vesicles possess a bilayer thickness of roughly $45 \mathrm{~nm}$ [23] as determined by small-angle neutron scattering (SANS). However, when supported, the bilayer thickness is known to increase in part due to an intermediate water layer between the underlying leaflet and substrate [63]. In this section, we will explore the effects of temperature and melittin concentration on the thickness variations of supported DMPC bilayers to better understand how these changes affect membrane fluidity.

\subsubsection{Sample Fabrication and AFM Operation}

Previous studies by Stroumpoulis et al. [13] have used phase-modulated ellipsometry to monitor and characterize the bilayer formation kinetics of DMPC onto silicon oxide surfaces via vesicle fusion. Figure 5.1 reveals how lipid concentration and deposition time affect the percent coverage of single supported bilayers. For the purposes of our study, only partial bilayer coverage was desired in order to leave sections of the substrate exposed so an accurate bilayer thickness could be measured. Therefore, 


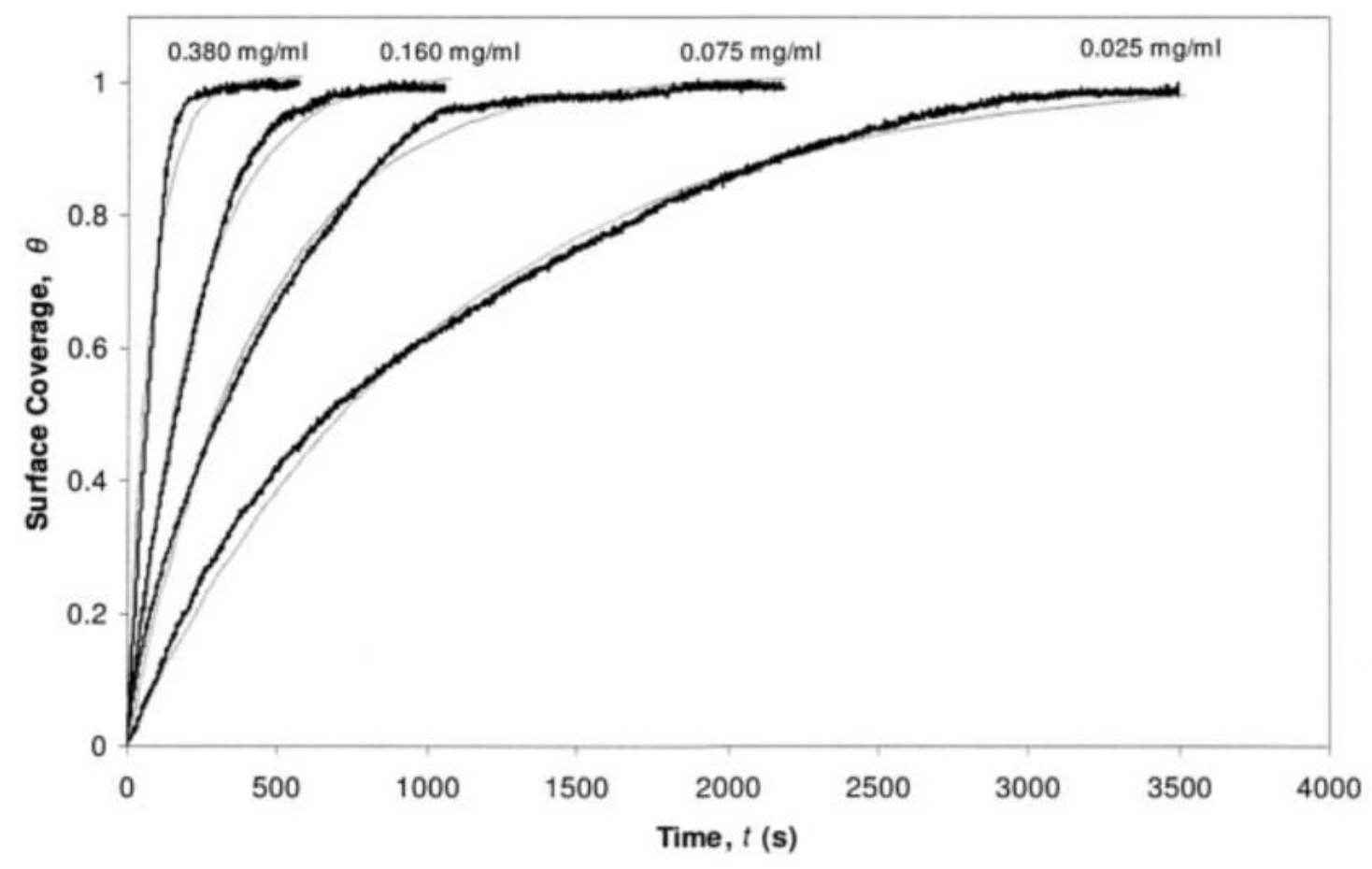

Figure 5.1: Formation kinetics of supported lipid bilayers of DMPC via vesicle fusion as a function of lipid concentration and deposition time determined by Stroumpoulis et al. [13]. Experimental data (dark black lines) were collected from phase-modulated ellipsometry and fitted (light gray lines) using a mass transport model. The surface coverage, $\theta$, represents the percentage of area occupied by DMPC bilayers with its rate increasing with higher lipid concentrations. Data were collected at $25^{\circ} \mathrm{C}$ for upwards of 1 hour at time intervals of 1 second following vesicle introduction. 
silicon oxide wafers were submerged in $50 \mathrm{ml}$ of $0.05 \mathrm{mg} / \mathrm{ml}$ DMPC lipid solution for 15 minutes at $60{ }^{\circ} \mathrm{C}$. The elevated deposition temperature ensures the lipid vesicles are in their fluid phase, thereby reducing the fusion time of the adsorbed vesicles and allowing satisfactory bilayer coverage in a short amount of time. To preserve partial bilayer coverage, no subsequent rinsing of the wafer was performed. Once the wafer emerged from the DMPC solution it was dried with $\mathrm{N}_{2}$ and immediately placed in the AFM for imaging. The melittin-treated sample, however, was transferred into a new solution containing the peptides after DMPC deposition.

For the same reasons mentioned above, it was also necessary for the melittintreated DMPC bilayers to have regions of exposed substrate. Therefore, once a wafer containing partially covered DMPC bilayers emerged from its solution it was immediately placed in a second solution containing a $0.5 \mu \mathrm{M}$ concentration of melittin peptides. The samples were then incubated for an additional 60 minutes at $60^{\circ} \mathrm{C}$ before being removed, dried with a gentle stream of $\mathrm{N}_{2}$, and imaged on the AFM. The peptide exposure time of 1 hour was found to be optimal for allowing melittin to insert into the predeposited DMPC membranes, while also conserving partial bilayer coverage. Peptide exposure times lasting longer than 1 hour resulted in a loss of most of the DMPC bilayers. In addition, it was found that melittin concentrations of 1.0 $\mu \mathrm{M}$ or greater would destroy many of the bilayers partially covering the wafer surface.

Temperature controlled operation of the AFM was conducted by slowly heating the AFM stage and tip simultaneously at a rate no greater than $0.5^{\circ} \mathrm{C} /$ minute using a multimode heater from Bruker. A special tip holder was used, which included a silicone rubber seal to isolate the sample environment for a more controlled atmosphere. The holder allowed for tip heating, gas and liquid purging, and an access for external 


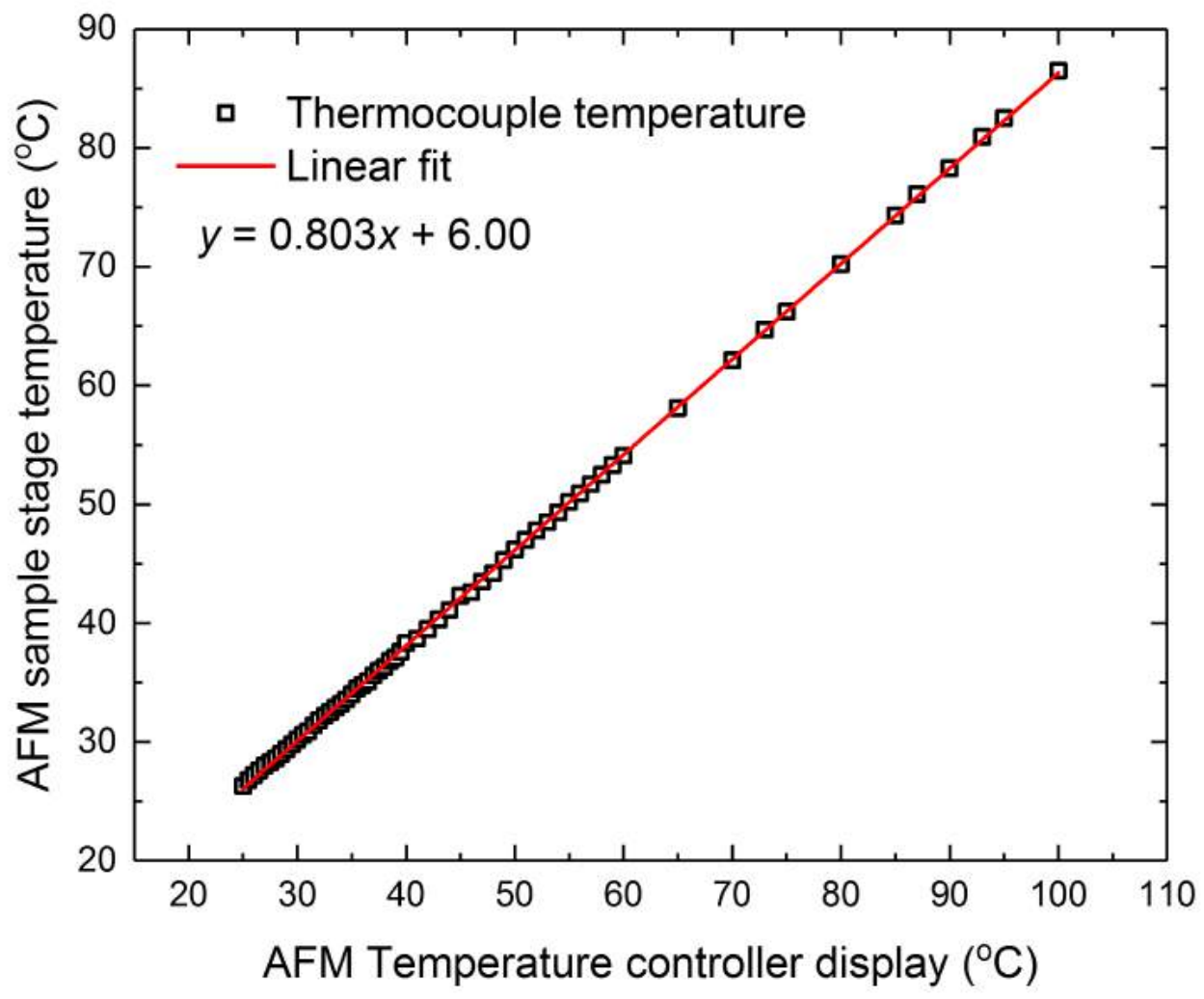

Figure 5.2: Temperature calibration curve obtained by placing a thermocouple in contact with the AFM stage while within the tip holder and plotting its reading against the temperatures displayed on the MultiMode heater from Bruker. A linear fit (red) was enforced to the data (black) and the resulting calibration function was used in calculating the actual temperatures during imaging. 
sensors such as a thermocouple, which was used to calibrate the temperature stage. Figure 5.2 shows the temperature calibration curve, which was used to calculate the actual temperatures during imaging. Images were obtained using a scan rate of 0.8 $\mathrm{Hz}$ with each line consisting of 256 pixels, resulting in a total collection time of 5.3 minutes per image. To allow for thermal equilibrium, two images were collected per temperature with the first and second images being scanned top down and bottom up, respectively. Data from the second images were then analyzed and reported on in the next section.

\subsubsection{Bilayer Thickness of Melittin-Treated DMPC Membranes as a Function of Temperature}

In this section, we explore the effects of melittin peptides on membrane thickness and fluidity of supported DMPC bilayers. Data present here were collected at the University of Missouri using AFM tips purchased from VISTAprobe with typical spring constants and resonant frequencies of $0.1 \mathrm{~N} / \mathrm{m}$ and $20-30 \mathrm{kHz}$, respectively.

Figure 5.3 shows two AFM images collected in air at $25^{\circ} \mathrm{C}$ using tapping mode, from bare DMPC membranes $(0.0 \mu \mathrm{M})$ and those treated with solution containing a melittin concentration of $0.5 \mu \mathrm{M}$. A short deposition time of 15 minutes allowed for the rupturing and fusing of DMPC vesicles to form many single-supported bilayers without completely covering the surface. Having incomplete bilayer coverage provides the advantage of accurately measuring membrane thickness via bearing and line trace analysis. The lack of additional bilayers in the melittin-treated DMPC membrane was a common occurrence and is believed to be a result of the wafer changing solutions, possibly washing away the smaller, remnant bilayers. 

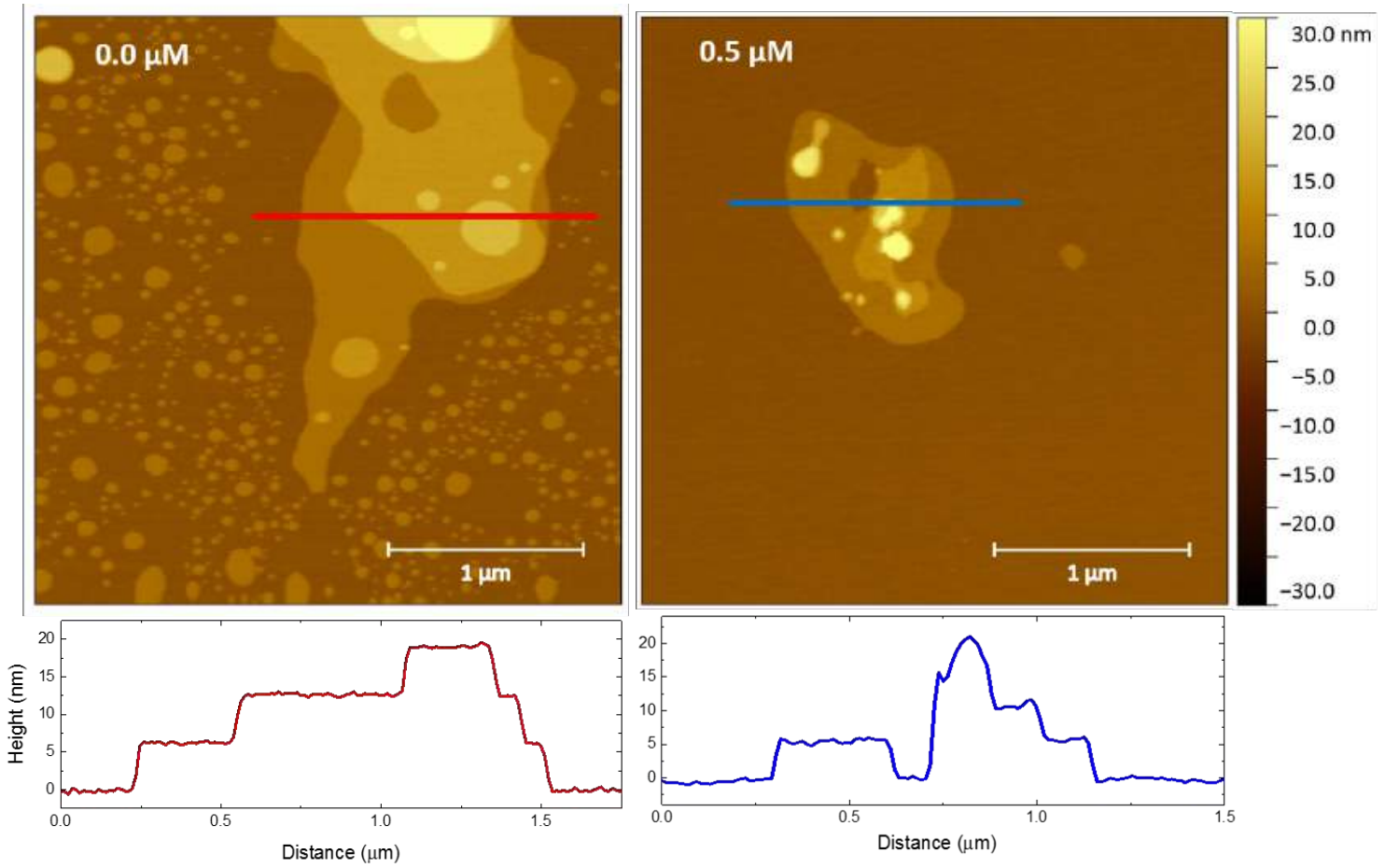

Figure 5.3: AFM images of bare DMPC bilayers (left) and those treated with $0.5 \mu \mathrm{M}$ melittin (right) supported on $\mathrm{SiO}_{2}$ as a result of short lipid deposition time collected on a Nanoscope IIIa instrument at the University of Missouri. Line trace analyses were performed on each multilayer feature shown in the AFM image over the solid red (blue) line for bare DMPC $(0.5 \mu \mathrm{M}$ melittin) with their respective profiles shown at the bottom of the figure. The line traces reveal well-defined step heights of ranging between $6.0-6.2 \mathrm{~nm}$, indicative of DMPC bilayers under these imaging conditions. Imaging was performed using tapping mode in air at $25^{\circ} \mathrm{C}$ and performed immediately after samples emerged from their solutions. 
Results from the line trace analysis performed on both bare and melittin-treated DMPC membranes can be seen in Fig. 5.3. The red and blue lines in Figure 5.3 (a) and (b), respectively, illustrate a section of the AFM images for which the height profiles were extracted. These sections were chosen because as the AFM tip traverses the membrane feature it encounters multilayers which remained after sample deposition. The height profiles at the bottom of Figure 5.3 reveal very well-defined step heights of equal distances on the order of $6.0 \mathrm{~nm}$ in the $z$-direction. These sharp steps in the line traces, which occur at the borders of each bilayer in the AFM image, are a testament to the accuracy of the measurement and the stability of the membranes under these imaging conditions.

Step heights recorded at $25{ }^{\circ} \mathrm{C}$ for the bare DMPC membranes shown in Figure 5.3 are roughly $6.2 \mathrm{~nm}$, which agree well with literature values for DMPC bilayers supported on glass substrates while in their gel-phase [63]. Although bare DMPC thicknesses on various substrates have been well-characterized, it is uncertain how the presence of melittin affects bilayer thickness or membrane stability. We see in Figure 5.3 that melittin-treated DMPC membranes possesses a similar thickness to the bare case while also maintaining their stability under these imaging conditions. However, it is not yet known how the presence of melittin effects the gel-to-fluid phase transition of DMPC when supported on a substrate.

To elucidate the full effect of melittin on the thicknesses of supported DMPC bilayers, temperature-dependent AFM measurements were performed in the temperature range $25^{\circ} \mathrm{C}<T<80^{\circ} \mathrm{C}$. By carefully heating the substrate supporting the membranes in temperature increments of $2-3{ }^{\circ} \mathrm{C}$, while simultaneously collecting AFM images, one is able to observe changes in bilayer thickness in real time. 
Figure 5.4 shows a series of AFM images recorded in air upon heating from bare DMPC membranes in the temperature range of $42{ }^{\circ} \mathrm{C}-52{ }^{\circ} \mathrm{C}$ using tapping mode. The white box in the leftmost image in Fig. 5.4 represents the area over which bearing analyses were performed to obtain height distributions for calculating bilayer thickness, the results of which can be seen in Fig. 5.7. At low temperatures, circular DMPC bilayers on top of the underlying membrane appear to remain stable and possess a thickness between $6.0-6.2 \mathrm{~nm}$ as expected. However, once heated to 50 ${ }^{\circ} \mathrm{C}$, the smaller DMPC bilayers which were present at $42{ }^{\circ} \mathrm{C}$ begin to fade away. Increasing the temperature further to $52{ }^{\circ} \mathrm{C}$ results in all of the additional bilayers that were once in the field of view to vanish completely. As these multilayer features are lost, the underlying bilayer increases in size, suggesting the bilayers that existed above it at low temperatures have now been absorbed. The absorption of DMPC bilayers indicates an increase in membrane fluidity and the onset of a gel-to-fluid phase transition. Interestingly, the temperature at which bare DMPC bilayers begin their absorption occurs at the same temperature that an initial decrease in bilayer thickness is observed (see Fig. 5.7). This correlation between bilayer absorption and decrease in membrane thickness provides strong evidence of a transition into the fluid phase, an effect not present in the melittin-treated case.

Unlike the bare DMPC membranes, those treated with $0.5 \mu \mathrm{M}$ melittin do not show any signs of bilayer collapse when heated to high temperatures. Figure 5.5 shows a series of AFM images of melittin-treated DMPC bilayers in the same temperature range as shown in Fig. 5.4 for the bare case. Here the melittin-treated bilayers appear to remain rigid with no correlation existing between changes in the AFM images and bilayer thickness. The fact that the melittin-treated bilayers do not collapse into the 


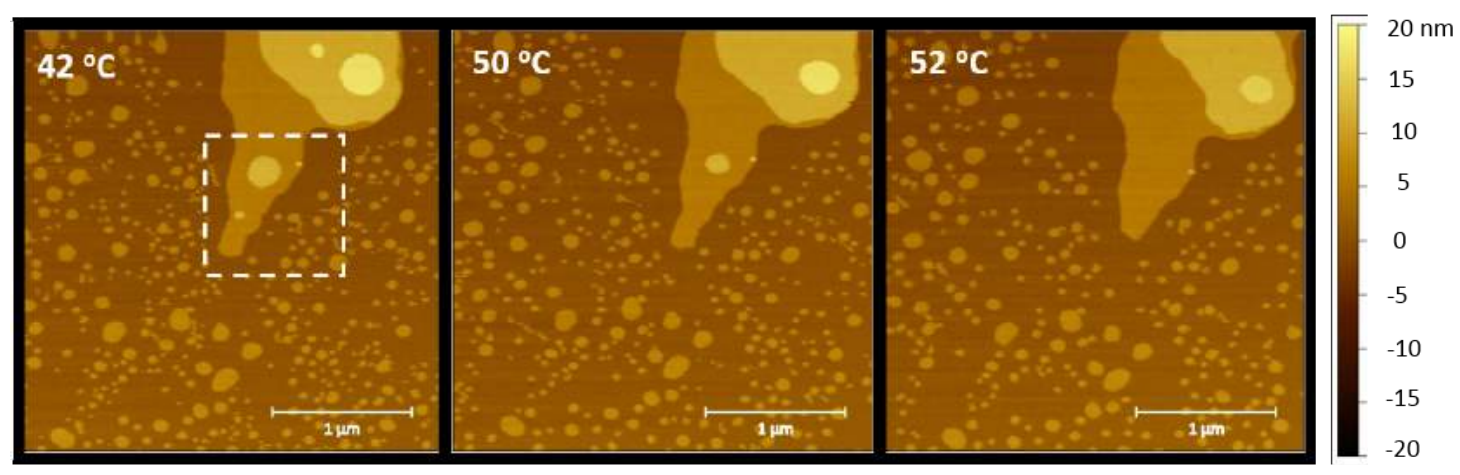

Figure 5.4: Progression of AFM images collected upon heating in the temperature range of $42{ }^{\circ} \mathrm{C}-52{ }^{\circ} \mathrm{C}$ from bare DMPC membranes. DMPC multilayers remain stable at low temperatures, but once a critical temperature of $50{ }^{\circ} \mathrm{C}$ is reached they begin to be absorbed into the underlying bilayer. This onset of bilayer absorption occurs at a temperature which correlates to an abrupt thickness decrease of the membrane. The white-dashed square shown in the left image represents the $1 \mu \mathrm{m}^{2}$ area over which bearing analyses were performed. Images were collected using tapping mode in air.

underlying layer at any of the temperatures measured suggests melittin peptides could be stiffening the membrane, preventing it from abruptly transitioning into a fluid-like state. Oriented circular dichrosim (OCD) experiments on DMPC vesicles have shown that melittin monomers can insert in the bilayer perpendicularly with respect to the membrane plane [58]. This particular peptide orientation would allow melittin to occupy significant space within the hydrophobic region of the membrane, thereby restricting motion of the lipid tails and causing a decrease in fluidity. Further evidence for decreased DMPC membrane fluidity due to melittin has also been observed via fluorescence measurements [64]. Although several studies using different techniques have shown melittin can induced a decrease in membrane fluidity, it is still not wellunderstood how this peptide might affect bilayer thickness of supported membrane systems. Therefore, to understand further how melittin affects the state of supported DMPC membranes, bearing analysis of the membrane heights has been conducted. 


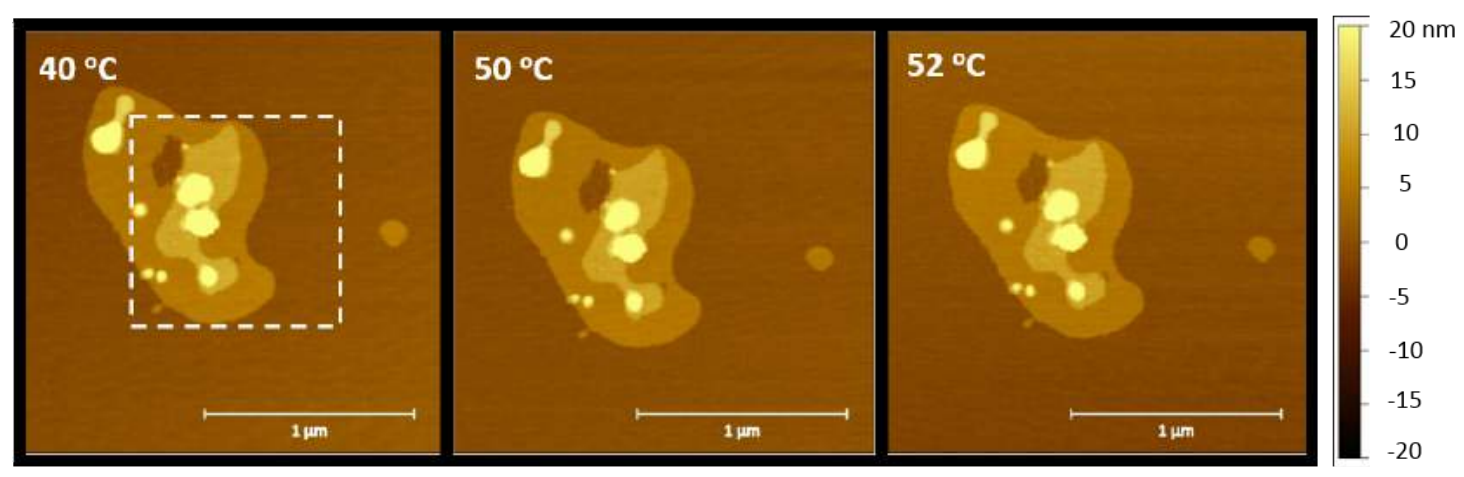

Figure 5.5: Progression of AFM images collected upon heating in the temperature range of $40^{\circ} \mathrm{C}-52^{\circ} \mathrm{C}$ from DMPC membranes treated with $0.5 \mu \mathrm{M}$ melittin. Contrary to the bare membrane case, multilayers of the melittin-treated membrane do not absorb into the underlying layer at any temperature. The white-dashed square shown in the left image represents the $1 \mu \mathrm{m}^{2}$ area for which bearing analyses were performed. Images were collected using tapping mode in air.

Quantifying bilayer thicknesses of samples of melittin-treated DMPC membranes is done via a bearing analysis, which bins individual pixels obtained from an AFM image according to their heights. From the resulting pixel height distribution, it becomes possible to extract information such as, bilayer percent coverage (integration under the peaks) and bilayer thicknesses (peak separation). For consistency, pixel height distributions were obtained from the same $1 \mu \mathrm{m}^{2}$ area depicted as the whitedashed square in Figures 5.4 and 5.5. These areas were chosen in part because of the greater than $50 \%$ coverage of bare $\mathrm{Si}$ (providing a reliable reference peak), and also due to the existence of multilayers within the analysis window. Having multilayers within the analysis window results in a multipeak distribution, allowing for the possibility to measure changes between thicknesses of the underlying bilayer and the upper ones. Figure 5.6 shows a single bearing analysis performed on the bare DMPC bilayer patch.

Results of bilayer thicknesses collected from melittin-treated DMPC membranes as a function of temperature are shown in Figure 5.7. The initial bare DMPC thickness 


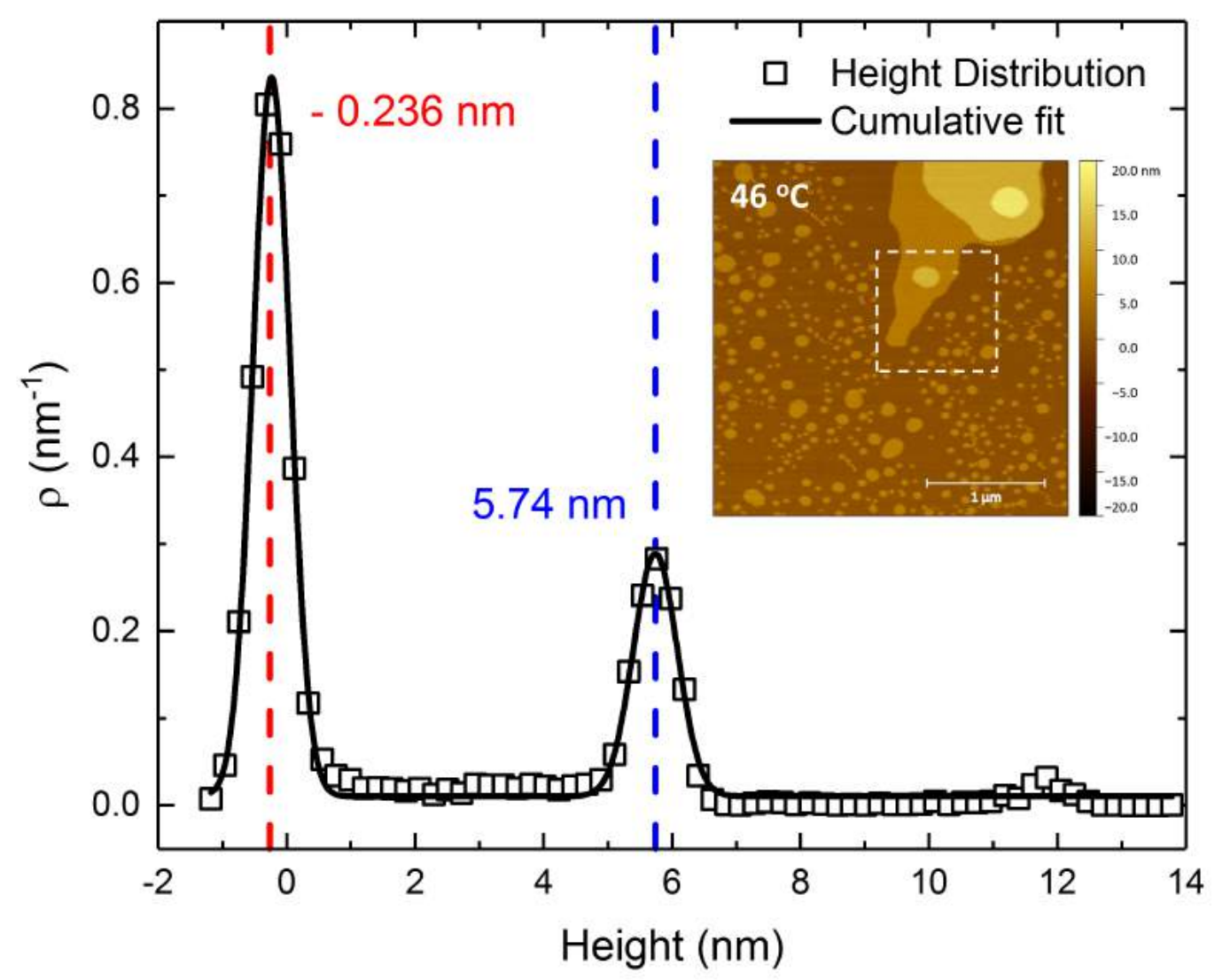

Figure 5.6: AFM pixel height distribution of DMPC membranes in the absence of melittin peptides collected at approximately $46{ }^{\circ} \mathrm{C}$ in air using tapping mode. The 1 $\mu \mathrm{m}^{2}$ area surveyed is represented by a white-dashed square in the inset that shows the source AFM height image. Analysis of the height distribution reveals a total of three peaks with the first being that of the Si substrate followed by the multilayer DMPC. The solid black line is a fit to the height distribution with the 3rd peak located at roughly $12 \mathrm{~nm}$ being omitted. 
of $6.2 \mathrm{~nm}$ agrees well with previously measured single-supported DMPC bilayers, and, upon heating it remains rather constant. Above a critical temperature of $52^{\circ} \mathrm{C}$, the bilayer thickness begins to decrease abruptly. This sharp reduction in membrane thickness indicates a transition from the stiffer gel phase to the softer fluid phase. Although the transition at $52^{\circ} \mathrm{C}$ occurs at a higher temperature than that of bare hydrated DMPC vesicles, single-supported DMPC membranes have been observed to undergo their gel-to-fluid phase transition at higher temperatures while in air [7]. As noted before, the temperature at which the sudden change in thickness occurs for the bare membrane correlates with the absorption of additional bilayers as observed from AFM images (see Fig. 5.4).

It can also be seen in Fig. 5.7 that no abrupt thickness change for the melittintreated membrane exists. Instead, the membrane thickness continuously decreases over the temperature range $30{ }^{\circ} \mathrm{C}<T<60{ }^{\circ} \mathrm{C}$. The lack of a well-defined gelto-fluid phase transition in the melittin-treated case suggests the proteins could be stiffening the membrane thereby decreasing its fluidity and broadening the transition. Both membranes are thought to be completely in their fluid phase at $60{ }^{\circ} \mathrm{C}$, as their thicknesses begin to plateau around $5.0 \mathrm{~nm}$. 


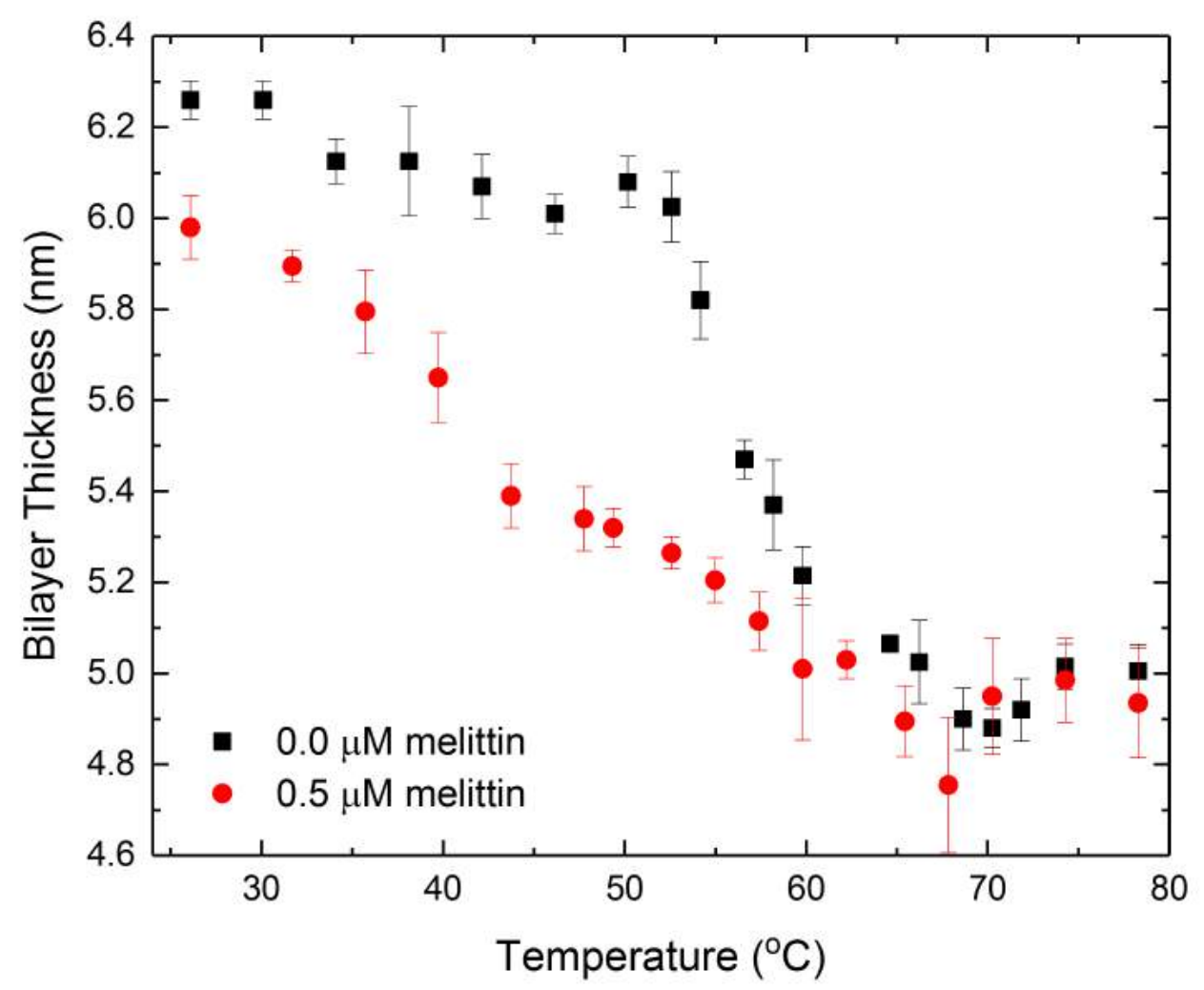

Figure 5.7: Temperature-dependent bilayer thicknesses of bare (black squares) and melittin-treated (red circles) DMPC membranes measured by AFM upon heating. The sharp decrease in thickness at $52{ }^{\circ} \mathrm{C}$ of bare DMPC indicates a first order gelto-fluid phase transition, which correlates with the absorption of DMPC multilayers seen in Fig. 5.4. There is no abrupt thickness change for melittin-treated membrane, but rather a continuous linear decrease is observed over the temperature range $30^{\circ} \mathrm{C}$ $<T<60{ }^{\circ} \mathrm{C}$. Both membranes reach a thickness of about $5.0 \mathrm{~nm}$ around $60{ }^{\circ} \mathrm{C}$. Thickness values were obtained from bearing analysis conducted over a $1 \mu \mathrm{m}^{2}$ area. 

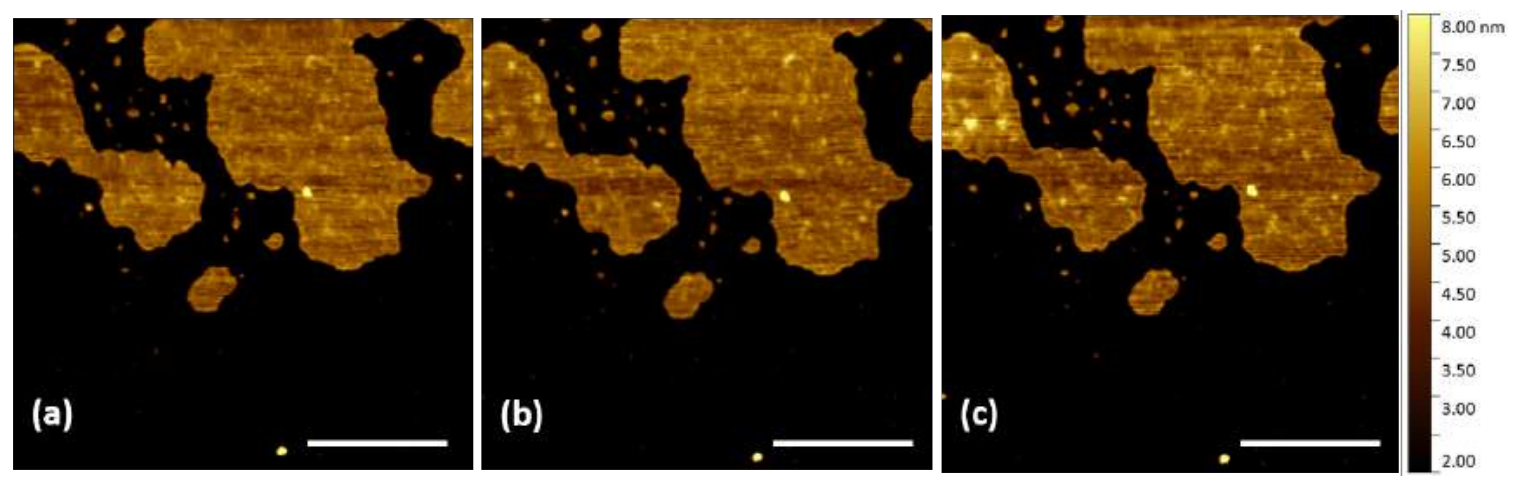

Figure 5.8: AFM images of a DMPC bilayer patch treated with $0.5 \mu \mathrm{M}$ melittin after being annealed at $55^{\circ} \mathrm{C}$ for (a) $127 \mathrm{~min}$, (b) $265 \mathrm{~min}$, and (c) $300 \mathrm{~min}$. After about 265 minutes, small clusters can be seen beginning to form on the bilayer surfaces. These are believed to be clusters of lipid-peptide complexes. AFM images were collected in air using tapping mode. Scale bar is $500 \mathrm{~nm}$.

\subsubsection{Time-Dependent Annealing Effects of Melittin-Treated DMPC Bilayers}

As mentioned in the previous chapter, there was an unexpected and curious timedependence observed in the elastic neutron intensity for several melittin-treated DMPC samples in the $0.5 \mu \mathrm{M}$ range as measured on the HFBS. In addition, AFM images had revealed the formation of large dimple-like features on the surfaces of melittin-treated DMPC (see Fig. 5.10) which appeared to materialize as result of annealing the membrane at $328 \mathrm{~K}\left(55^{\circ} \mathrm{C}\right)$. It was therefore postulated that membrane-bound melittin peptides could be aggregating during the annealing annealing process to form large impressions in the bilayer. To help elucidate the cause for such behavior, we have performed AFM measurements at $328 \mathrm{~K}\left(55^{\circ} \mathrm{C}\right)$ and observed structural changes in the bilayer as a function of time.

Figure 5.2.3 shows a series of AFM images collected over 300 minutes from DMPC patches treated with a solution containing $0.5 \mu \mathrm{M}$ melittin. Initially, the bilayer 


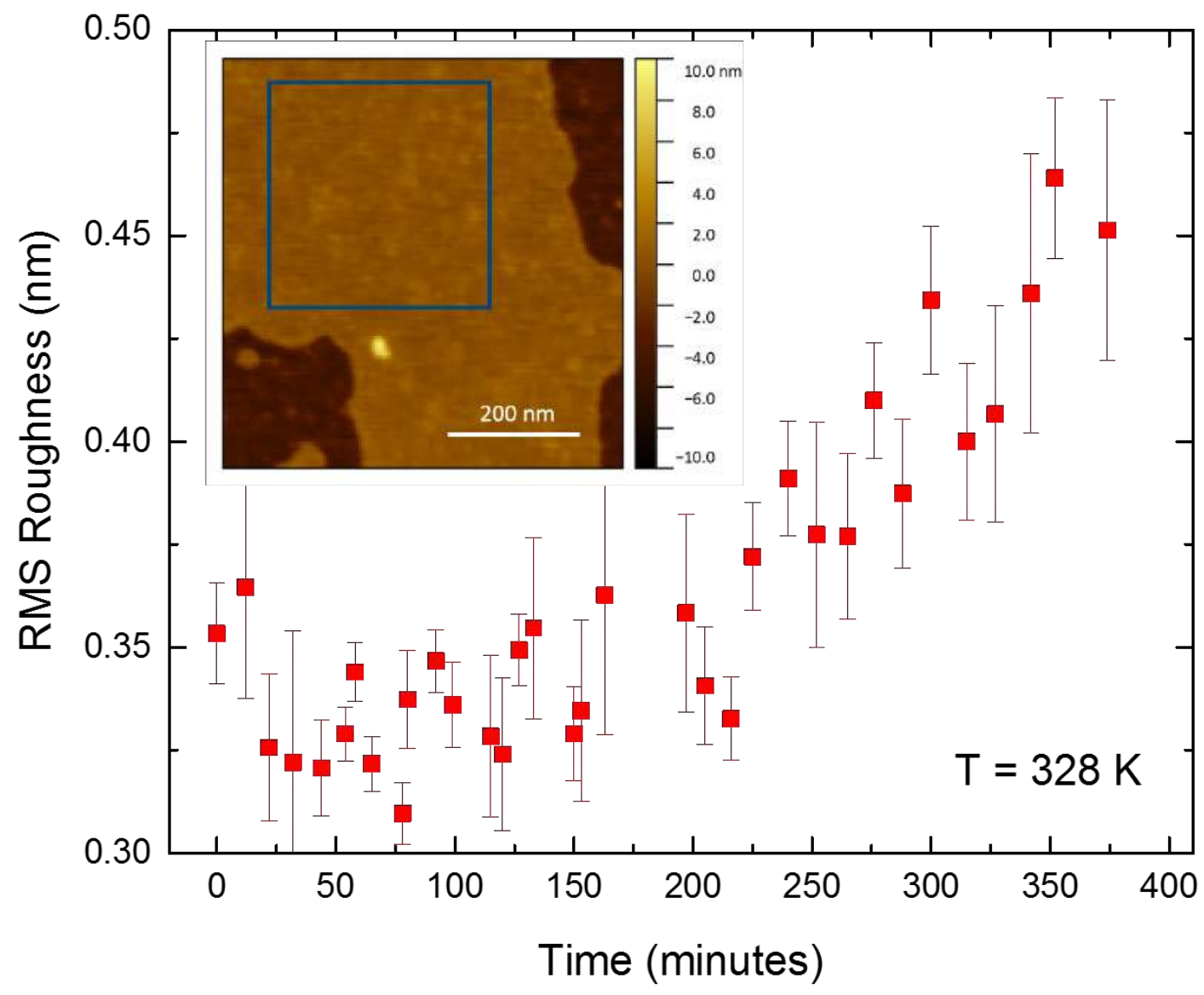

Figure 5.9: Time-dependent roughness of a $0.5 \mu \mathrm{M}$ melittin-treated DMPC bilayer during an anneal at $328 \mathrm{~K}$ determined by AFM. The inset is an AFM image of the bilayer patch which was investigated, with the blue box representing the area for which roughness values were obtained. 
patches appear uniform and free of defects or dimples (data not shown). However, after about 127 minutes of annealing the sample at $328 \mathrm{~K}$, small changes in the surface roughness of the DMPC patch are observed and cluster particles begin to form. After an anneal duration of 300 minutes, the sample surface is covered with small nearly uniformly sized particles believed to be clusters of lipid-peptide complexes. The melittin treated DMPC surface roughnesses were also analyzed as a function of anneal duration. These results were then correlated to the formation of clusters appearing in the AFM images shown in Fig. 5.8.

Figure 5.9 shows the results from surface roughness measurements performed on AFM images collected from a $0.5 \mu \mathrm{M}$ melittin treated DMPC bilayer patch as a function of anneal duration. The inset in Fig 5.9 is of a bilayer patch used to extract root mean square (RMS) roughness values. The bilayer patch that appears in the inset of Fig. 5.9 is the same patch that appears in Fig. 5.8. The roughness values of the melittin-treated DMPC bilayer patch calculated by analyzing the AFM images remains roughly constant near $0.325 \mathrm{~nm}$ for the first 120 mins of annealing at $328 \mathrm{~K}$. However, once anneal time exceed 150 mins, there is a clear increase in the roughness values, indicating possible peptide aggregation. The RMS roughness continues to increases linearly up until the end of the measurements at 380 minutes. 


\subsection{In situ AFM Measurements on Melittin-Treated DMPC Bilayers}

To visualize the structural changes occurring within a membrane as a result of protein exposure, in situ AFM measurements were performed on single-supported DMPC bilayers in the presence of melittin. These measurements helped aid in the interpretation of the membrane morphology for melittin-treated DMPC samples used in our neutron scattering studies. Images presented in this section reveal the formation of dimple-like features on the membrane surface induced by melittin, which coalesce into larger domains when annealed at $328 \mathrm{~K}$ without compromising membrane integrity. These features are coincident with time-dependent increases of the elastic neutron intensities as discussed earlier in Section 5.2.3, suggesting aggregation of membrane-bound melittin peptides.

\subsubsection{Sample Preparation for In situ Study}

Sample fabrication, for in situ samples, began by using a vesicle fusion method similar to that described previously (see Chapter 1) to deposit uniform, single-supported, bare DMPC membranes. The lipid concentration ( 100 $\mu \mathrm{g} / \mathrm{ml}$ DMPC) and ionic strength of its solution ( $5 \mathrm{mM}$ HEPES, $5 \mathrm{mM} \mathrm{MgCl}_{2}$, and $100 \mathrm{mM} \mathrm{KCL}$ ) were identical to samples fabricated for neutron scattering measurements; however, some modifications were made to the deposition process. Recall that all neutron scattering samples were deposited onto $\mathrm{SiO}_{2}$-coated single-crystal silicon wafers which were completely submerged in lipid solution during the incubation period. For AFM studies, the deposition and subsequent bilayer formation of DMPC occurred in a 
temperature-controlled petri dish under humid conditions with a mica puck serving as the substrate.

A small mica substrate ( $\sim 12 \mathrm{~mm}$ in diameter) was glued to the top of a teflon spacer supported on a magnetic puck designed to fit into the AFM sample holder. This stacking method provided enough space between the mica surface and magnetic puck to support a stable buffer droplet without risking it making contact with the base. The mica surface was then cleaved multiple times with scotch tape before being placed in a petri dish, which sat on a hot plate held at $30{ }^{\circ} \mathrm{C}$. A small paper towel was dipped in deionized water and placed in the petri dish to maintain a humid environment.

After allowing the temperature to equilibrate within the petri dish, a $120 \mu \mathrm{l}$ droplet containing small unilamellar vesicles of DMPC at a lipid concentration of $100 \mu \mathrm{g} / \mathrm{ml}$ was carefully deposited onto the mica surface. The petri dish was then isolated and the solution allowed to incubate for 30 minutes. During this time the droplet was observed to evaporate slightly; however, the high humidity within the petri dish helped minimize this effect. After 30 minutes, the remaining lipid solution was extracted using a pipette and replaced with a $100 \mu \mathrm{l}$ droplet of fresh buffer. This fluid exchange of buffer was repeated 8 times to wash away additional DMPC bilayers that may have formed.

Once the fluid exchange was complete, the magnetic puck supporting the mica and freshly deposited DMPC membrane was transferred to the AFM sample stage, which was also held at $30^{\circ} \mathrm{C}$. A new buffer droplet was then introduced to the membrane in addition to the AFM tip before carefully making tip contact with the surface. Several images were then collected to confirm complete DMPC bilayer coverage. Upon 
confirmation of bilayer coverage, the tip was retracted and buffer solution replaced with a droplet containing melittin peptides. Once the melittin solution was place onto the DMPC membrane, contact was again made and in situ AFM image collection began.

\subsubsection{Melittin-Induced Dimple Formation in DMPC Bilayers}

Figure 5.10 shows two AFM images collected at $303 \mathrm{~K}$ under buffer solution from a melittin-treated DMPC bilayer supported on a mica substrate (a) 0 minutes and (b) 60 minutes after exposure to a peptide concentration of $1.0 \mu \mathrm{M}$. Initially, a featureless DMPC bilayer is observed, with an average surface roughness slightly greater than that of the mica substrate $(<0.1 \mathrm{~nm})$. After 60 minutes of melittin exposure, a number of dimple-like features can be seen on the DMPC surface (see Fig. 5.10(b)), which occupy roughly $6-10 \%$ of the total scan area. Line trace analysis over the dimples reveal diameters in the range of 60 - $80 \mathrm{~nm}$ and depths of roughly $1 \mathrm{~nm}$. Upon further exposure, the dimple-like features remained stable; however, after about 80 minutes, the buffer solution had evaporated resulting in unstable imaging conditions.

The lower two panels in Fig. 5.10 show the effect of annealing on DMPC membranes treated with a solution containing $1.0 \mu \mathrm{M}$ melittin. Unlike those in the upper panels, these AFM images were taken in the absence of buffer solution and at a slightly lower temperature of $295 \mathrm{~K}$. Prior to annealing, we again observe dimple-like features on the membrane surface as in Fig. 5.10(b), although the longer exposure time of 4 hours and drier imaging conditions yield larger diameters ( $\leq 200 \mathrm{~nm}$ vs. $\sim 70 \mathrm{~nm})$. Despite the drier state of the membrane, the diameters increase with annealing up to $500 \mathrm{~nm}$ (see Fig. 5.10(d)), but the dimples maintain about the same depth of $\sim 1 \mathrm{~nm}$ 

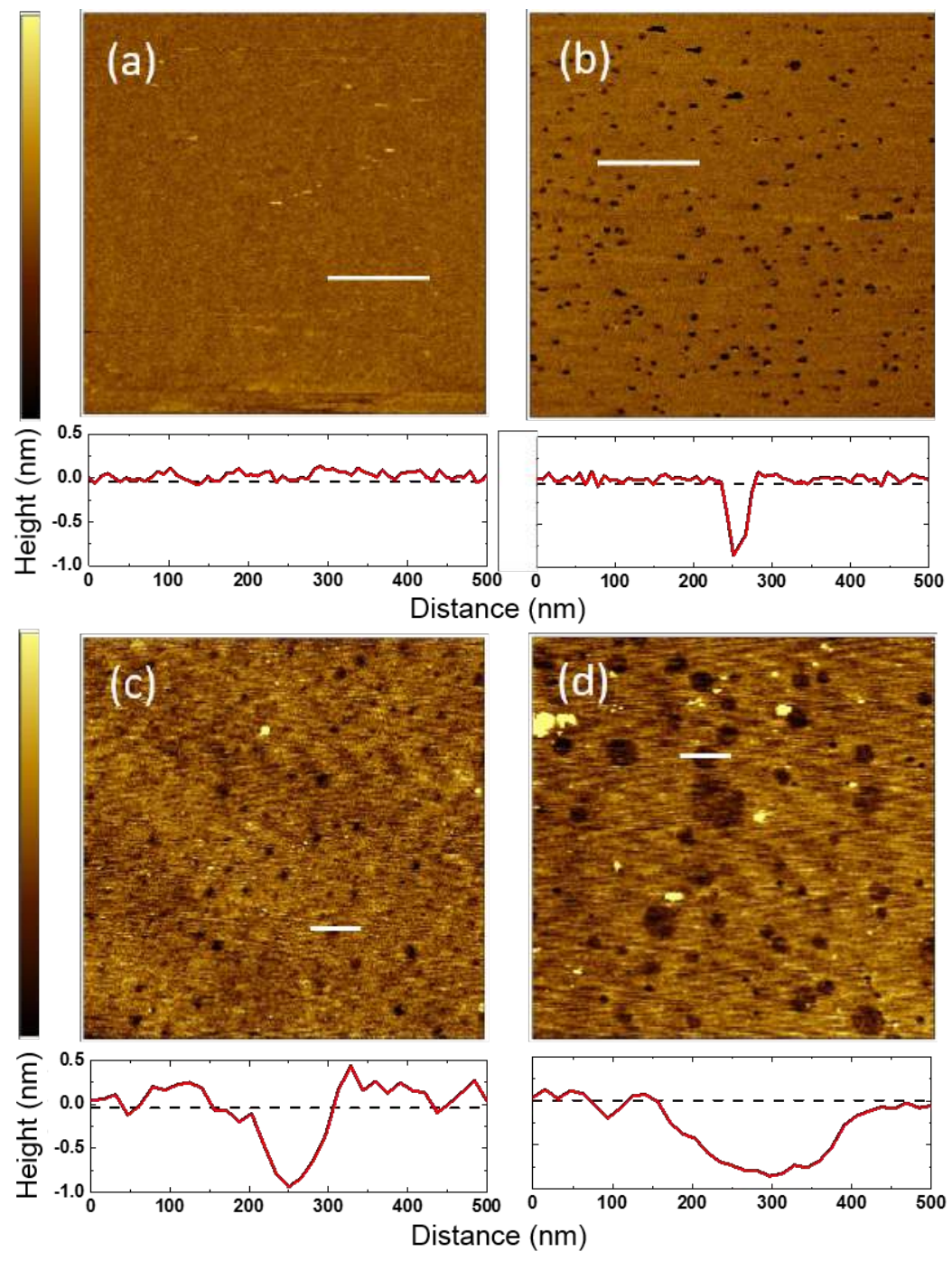

Figure 5.10: AFM images and line scans of $1.0 \mu \mathrm{M}$ melittin-treated DMPC supported bilayers. The upper panel displays in situ images of a DMPC membrane deposited on a mica substrate collected under buffer at $303 \mathrm{~K}$ (a) 0 minutes and (b) 60 minutes after an exposure to melittin. AFM images in the bottom panel were collected in air at $295 \mathrm{~K}$ from samples deposited on silicon wafers (c) before and (d) after an anneal at $328 \mathrm{~K}$. Line scans were performed over the $0.5 \mu \mathrm{m}$ white scale bar shown in the images and reveal depths of roughly $1 \mathrm{~nm}$ for all dimple-like features. Upon annealing (d) the dimple diameters increase to as large as $500 \mathrm{~nm}$ without causing membrane destruction. All images have a $\mathrm{z}^{-}$-scale of $1 \mathrm{~nm}$ and were collected in tapping mode using tips with radii $<10 \mathrm{~nm}$. 
as those observed initially and in the in situ case.

The anneal temperatures for our melittin-treated samples were determined from previous AFM measurements of the temperature dependence of the DMPC bilayer thickness that showed a transition into its fluid phase beginning at $328 \mathrm{~K}\left(55^{\circ} \mathrm{C}\right)$ while in air (c.f. Fig. 5.7). Although during its anneal the membrane is in a dehydrated state, the lipid motion is more rapid at these higher temperatures. This increased mobility is believed to facilitate the aggregation of surface-bound melittin, a hypothesis supported by measurements of the incoherent elastic neutron scattered intensity as a function of time (c.f. Fig. 4.5).

Previous studies suggest that, prior to forming transmembrane pores, melittin and other AMPs can induce thinning of the membrane [65-67]. Mecke et al. [68] performed AFM measurements on supported DMPC bilayers treated with an AMP similar to melittin at concentrations of $1-10 \mu \mathrm{M}$. They also observed formation of distinct domains of a peptide-lipid phase of a well-defined thickness less than that of the surrounding bilayer and which was independent of the total amount of peptide bound to the membrane [68]. The average height difference of these domains was found to be $1.1 \pm 0.2 \mathrm{~nm}$, which is comparable to the $\sim 1 \mathrm{~nm}$ depth of the dimplelike features in Fig. 5.10. In addition, Rakowska et al. [69] have reported AFM images collected in air from a supported PC bilayer treated with a synthetic AMP that reveal circular impressions in membrane as large as $10 \mu \mathrm{m}$ in diameter and depths of less than $1 \mathrm{~nm}$ as a result of its exposure to a $10 \mu \mathrm{M}$ peptide solution.

We analyzed AFM images of samples similar to those used for our QENS measurements following the procedure that Mecke et al. used to infer a peptide-to-lipid ratio $P / L[68]$. Due to the subtle changes in heights between dimple-like features and bare 
membrane, bearing analyses of the AFM images in Fig. 5.10 were unable to produce a bimodal distribution. Therefore, to estimate a $P / L$ for our samples, the associated areas for each pixel were summed up based on whether they belonged to dimple or not. Knowing the total area occupied by the dimples, we are then able to calculate a rough $P / L$ under the following assumptions: 1) the melittin peptides are bound to the DMPC membrane with their helical axis parallel to the bilayer plane $[45,53] ; 2$ ) the peptide and the lipid occupy a cross-sectional area of $4 \mathrm{~nm}^{2}$ [53] and $0.6 \mathrm{~nm}^{2}$ [68], respectively; 3) within a dimple, the peptides completely displace lipids from the upper leaflet, as proposed by Mecke et al. [68]; and 4) there are a negligible number of peptides outside the dimples. The constancy observed for the dimple depth supports assumption 3). If a varying number of lipids remained in the upper leaflet within the dimple areas, we might expect a distribution of dimple depths. With regard to assumption 4), we note that the presence of peptides between dimples would increase our estimate of $P / L$; however, they are likely to exist as isolated monomers or small clusters too small to participate in pore formation. We note that the smallest pixel size in Fig. 5.10 corresponds to a distance $<8 \mathrm{~nm}$ per pixel. Therefore, neither AFM set-up had sufficient lateral resolution to allow imaging of individual melittin monomers.

Under these assumptions, we estimate a maximum peptide-to-lipid ratio $(P / L)$ of $\sim 1 / 120$ for the $1.0 \mu \mathrm{M}$ melittin-treated sample in Fig. $5.10(\mathrm{~d})$ and $\sim 1 / 300$ for a similarly prepared $0.5 \mu \mathrm{M}$ sample. Despite the uncertainty in these estimates, we conclude that our SSLB QENS sample made with a $0.5 \mu \mathrm{M}$ melittin concentration is substantially below the critical value of $P / L^{*}=1 / 45$ determined for pore formation in DMPC multilayer membranes exposed to melittin [45]. Therefore, the analysis of 

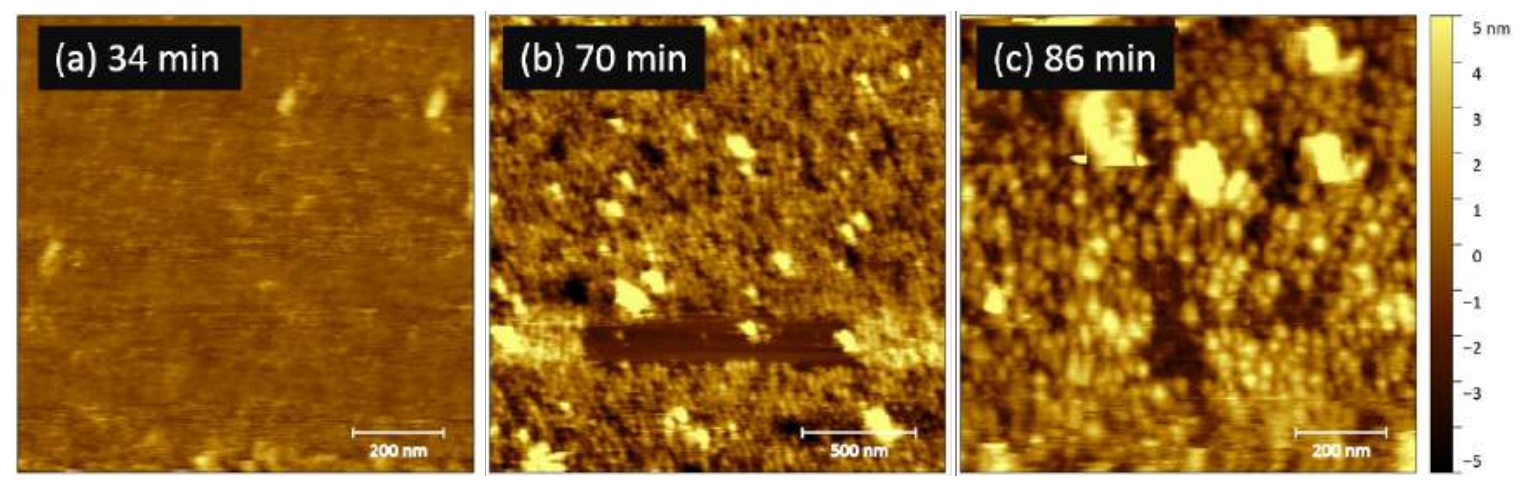

Figure 5.11: Time-dependent in situ AFM images of a DMPC membrane treated with a $4.0 \mu \mathrm{M}$ melittin solution collected under buffer at $30{ }^{\circ} \mathrm{C}$. After 34 minutes (a) of peptide exposure, the DMPC membrane remains stable and free of any visible aggregates or pores; however, at 70 minutes (b) clear morphological changes can be seen. Large holes in the membrane measuring 3-5 $\mathrm{nm}$ deep are present, in addition to globular-like features. At 86 minutes (c) membrane integrity is lost. Images were collected at the CNMS and have a $\mathrm{z}$-scale of $5 \mathrm{~nm}$.

our QENS spectra described in Chapter 4, considered the possibility of water confined to pores for a $0.5 \mu \mathrm{M}$ melittin-treated sample to be unlikely; however, their presence could not be completely ruled out.

DMPC bilayers treated with melittin concentrations greater than $1.0 \mu \mathrm{M}$ were also investigated by AFM. However, their surfaces were found to be highly disordered with insufficient homogeneity for neutron scattering measurements. Figure 5.11 shows AFM images collected in situ from a DMPC membrane treated with a $4.0 \mu \mathrm{M}$ melittin solution. After the introduction of melittin, the DMPC surface was carefully imaged over 90 minutes at which time morphological changes were clearly observed.

Figure 5.11(a) was collected after 34 minutes and shows a slightly rough, but intact DMPC membrane, free of any visible aggregates or pores. However, after 70 minutes of being exposed to $4.0 \mu \mathrm{M}$ melittin (see Fig. 5.11(b)), the DMPC membrane becomes highly disordered, revealing voids and large globular-like features littered about its 
surface. The globular-like features are believed to be protein-lipid complexes liberated from the membrane as a result of its disintegration. These observations are consistent with the detergent model for AMP protein insertion (see Fig. 4.1). After 86 minutes (see Fig. 5.11(c)) the membrane integrity has been completely compromised and destruction of the bilayer can be seen. Imaging was terminated after 90 minutes due to AFM tip contamination and the evaporation of the melittin buffer droplet. These AFM images suggest that greater melittin concentrations will result in the destruction of single-supported DMPC bilayers. Therefore, we limit our neutron scattering studies to melittin concentrations known to maintain membrane integrity.

\subsection{Methods of Confirming Bilayer Coverage}

We have seen in this chapter that single-supported lipid bilayers of DMPC are extremely thin structures, possessing thicknesses of $<6 \mathrm{~nm}$, which can be difficult to probe with methods other than AFM. However, neutron reflectivity is a powerful tool capable of structural characterization on such length scales and has been used to measure thicknesses of DMPC bilayers [70]; but, this technique often requires extremely large sample areas and high neutron flux to achieve satisfactory statistics. In this section, we will explore alternative complementary methods used to confirm the presence of a single-supported lipid bilayer.

\subsubsection{Force Approach Curves}

The applications of AFM are not limited to topographical imaging. Additionally, AFM is capable of detecting the mechanical properties of a surface using force ap- 
proach curve (FAC) techniques. Force approach curves are an extremely valuable tool for studying biological processes and have been used to elucidate forces associated with protein folding near a membrane interface [71]. A FAC is performed by first eliminating any lateral motion of the sample with respect to the tip and carefully decreasing the $z$ distance, while simultaneously measuring the cantilever deflection. Once contact between the tip and sample surface is established, the tip is then retracted from the surface. By monitoring the cantilever deflection as a function of distance from the sample surface upon approaching/retracting the tip, one can infer characteristics of the tip-surface interactions and the forces associated with them.

Due to the planarity and extremely smooth surfaces of a supported lipid bilayer, AFM images alone may not be sufficient enough to distinguish its surface from the substrate. Therefore, FACs are useful for comparing hard surfaces, such as mica and $\mathrm{SiO}_{2}$, to the softer and more elastic surfaces of a lipid membrane. In addition to FACs, roughness measurements of the surface can also provide convincing evidence that a bilayer is present.

Figure 5.12 shows AFM images from a mica and DMPC surfaces collected under buffer along with their average roughness values and force approach curves. In the case of mica, the approach curve (black curve in Fig. 5.12(a)) shows a subtle undulation in the cantilever deflection upon making contact with the surface. When the tip is subsequently retracted from the mica surface (red), the cantilever deflection reaches a minimum value before experiencing an abrupt increase, indicating a quick release of the tip from the surface. This FAC profile of the mica surface is indicative of a "hard" weakly interactive surface. Moreover, the average roughness calculated from the AFM image was found to be $27.7 \mathrm{pm}$, confirming an atomically smooth surface. In contrast, 

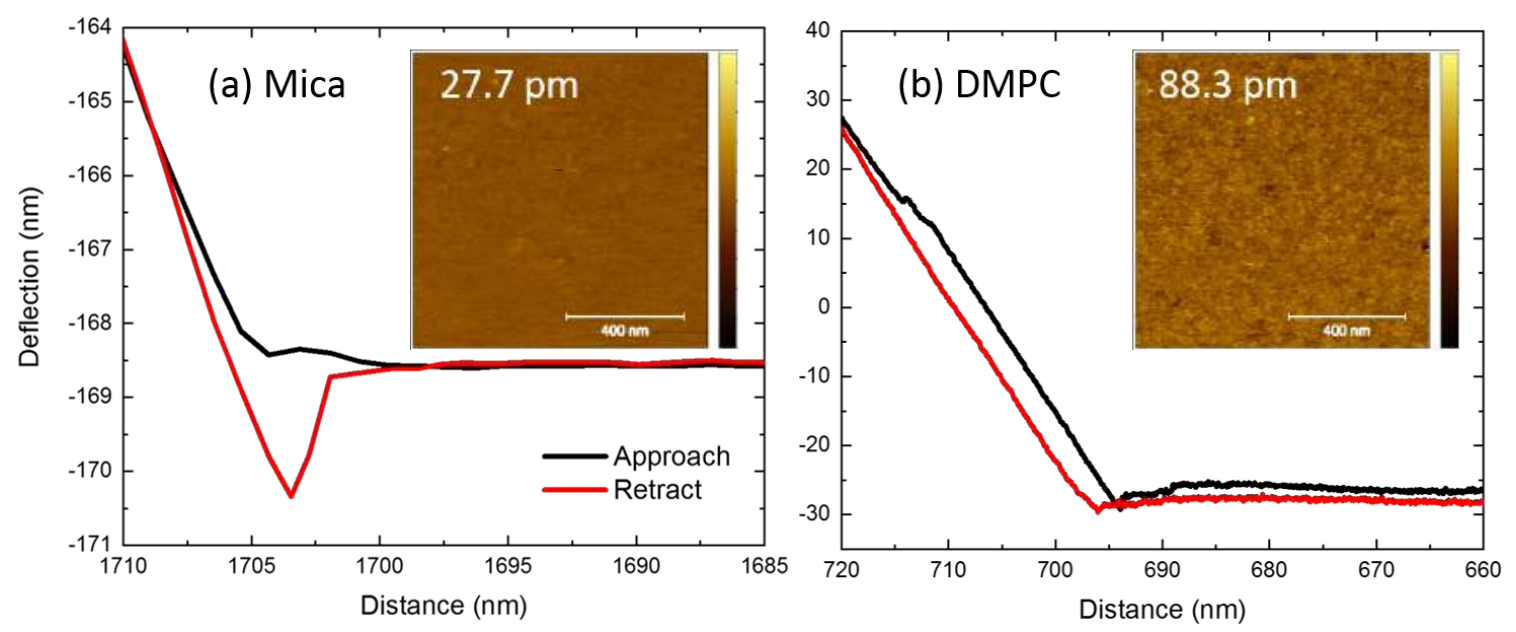

Figure 5.12: Force approach curves for mica (a) and DMPC (b) collected at the CNMS under buffer using tapping mode at $T=295 \mathrm{~K}$. The inset in each plot a 1 $\mu \mathrm{m}^{2}$ AFM image of the sample surface. Average roughness values of $27.7 \mathrm{pm}$ and $88.3 \mathrm{pm}$ were calculated over the entire mica and DMPC image, respectively. Both AFM images have a $z$-scale of $1 \mathrm{~nm}$.

roughness values and FACs obtained from a DMPC membrane are consistently larger than those observed for mica.

The DMPC FAC (see Fig. 5.12(b)) initially shows a downward deflection as the tip approaches the membrane before being deflected upward in a linear fashion. As the tip-surface distance is decreases further, the approach curve (black) deviates from its linear behavior before full contact is made, as indicated by the convergence of approach and retract curves. Upon retraction, the tip deflection remains rather constant before experiencing minor fluctuations as it completely departs from the membrane surface. The average roughness of the DMPC surface yielded values nearly 3-fold greater than for mica. A slightly rougher surface than the substrate is to be expected for the supported membrane.

Typically, full bilayer coverage is desired for SSLBs measured by neutron scatter- 
ing; however, as we have seen earlier in this chapter, it is sometimes favorable to have incomplete bilayer coverage for thickness measurements. In order to explore further the capabilities of FACs, multilayer DMPC samples were fabricated using methods similar to those described previously (see Chapter 1 ). The only difference in fabricating multilayer DMPC samples was the incubation time and temperature. A Si wafer was submerged in a $100 \mu \mathrm{l}$ DMPC solution held at $45{ }^{\circ} \mathrm{C}$ for $<5$ minutes. Upon removal from the solution, the $\mathrm{Si}$ wafer was not rinsed and immediately transferred to the AFM sample stage. The resulting images collected from the surface revealed the formation of DMPC multilayers.

Figure 5.13 shows an AFM image containing DMPC multilayers and a FAC collected from the multilayer feature. The location at which the FAC was collected is indicated by a red $\mathrm{X}$ in Fig. 5.13, which appears to be on top of at least three underlying bilayers. The approach curve (black) indicates weak initial attraction to the surface before the cantilever begins an upward deflection. As the tip-surface distance decreases further, there are three subtle regions of the approach curve that deviate from linearity, each represented by a small plateau (see inset in left panel of Fig. 5.13). Measuring the distances over which these plateaus occur reveal thicknesses on the order of $5 \mathrm{~nm}$, which are comparable to the thickness of a DMPC bilayer. Therefore, not only are the FACs able to reveal useful information regarding the mechanical properties of a surface, but can also provide quantitative results complementing those obtained from topographical imaging. 

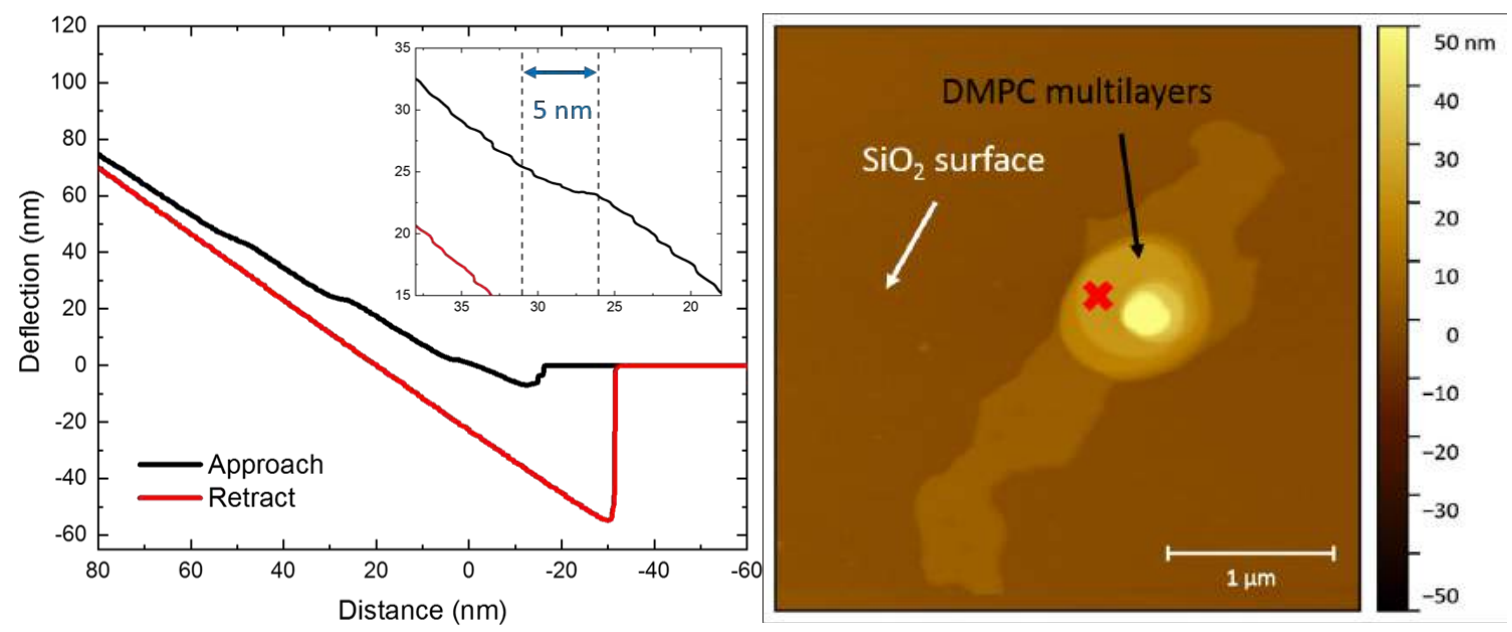

Figure 5.13: Force approach curves (left) and AFM image (right) collected under ambient conditions in tapping mode at the CNMS from DMPC multilayers supported on Si. The spot at which the FAC data was collected is located on top of several DMPC bilayers and depicted as a red X in the AFM image. As the distance between sample and tip is decreased, the approach curve (black) shows an overall linear trend in the cantilever deflection with three subtle, equally spaced plateaus. The inset is a zoomed in region of a plateau, with vertical dash lines representing points at which the distances in the approach curve deviate from linearity. The plateau distance is measured to be $\sim 5 \mathrm{~nm}$, which is comparable to the thickness of a DMPC bilayer. 


\subsubsection{Raman Spectroscopy}

Raman spectroscopy is an inelastic light scattering technique which can be used to identify specific molecular species from detection of vibrational, rotational, and other low-frequency dynamical modes. Here we show how Raman spectroscopy can be used to confirm the presence of lipid molecules from the unique frequency of the $\mathrm{C}-\mathrm{H}$ stretching mode originating in the alkane tails. Lee et al. [72] have demonstrated, using Raman spectroscopy, that it is possible to detect the temperature-dependent vibrational spectra of this $\mathrm{C}-\mathrm{H}$ stretching mode in the supported DMPC bilayers. They found that the C-H stretching mode for supported DMPC bilayers near $296 \mathrm{~K}$ occurs at roughly $2900 \mathrm{~cm}^{-1}$.

Figure 5.14 shows the Raman spectra collected from a bare Si wafer wafer (black curve) and one which had been deposited with DMPC (red curve). The spectrum for $\mathrm{Si}$ is mostly featureless, indicating no Raman active frequencies in this region. However, the spectrum obtained from the DMPC sample shows two main features, indicating the presence of several molecular vibrational modes. The first peak, centered at $3200 \mathrm{~cm}^{-1}$, is quite broad and represents an overlap of the vibrations modes belonging to the $\mathrm{O}-\mathrm{H}$ bond in water. The second feature appears to be a cluster of

sharper peak is centered at $2900 \mathrm{~cm}^{-1}$, providing evidence that the surface contains significant amount of lipids to produce a $\mathrm{C}-\mathrm{H}$ stretching mode signal. 


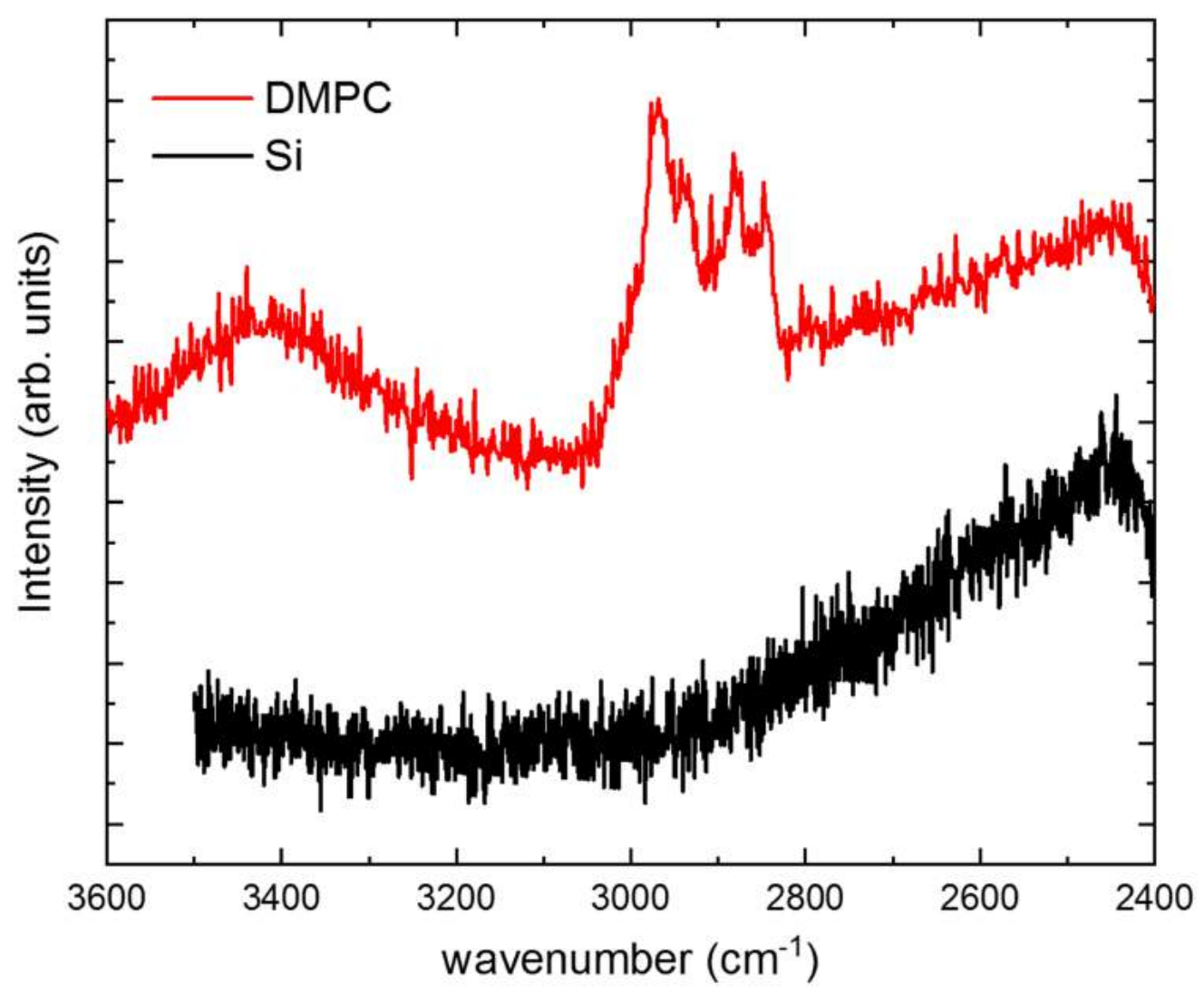

Figure 5.14: Raman spectra from a bare silicon wafer (black) and DMPC bilayers (red) collected under ambient conditions using an incident photon wavelength of 532 $\mathrm{nm}$. Scans have been shifted for clarity. A series of peaks near wavenumber 2900 $\mathrm{cm}^{-1}$ are associated with the $\mathrm{C}-\mathrm{H}$ stretching vibrational mode in the DMPC lipid alkane tails. The broad peak centered at $3200 \mathrm{~cm}^{-1}$ is an overlap of the vibrations modes for the $\mathrm{O}-\mathrm{H}$ bond in water. 


\section{Appendix A}

\section{Schematic of Sample Cell used in}

\section{Neutron Scattering Measurements}

In order to achieve an appreciable signal to noise ratio for our neutron scattering measurements on hydrated single-supported membranes, a large sample volume was desired. To fulfill this requirement, custom aluminum sample cells were designed in Autocad and fabricated at the University of Missouri physics machine shop. When sealed, these custom cells have total inner volume of about $72 \mathrm{~cm}^{3}$, which is large enough to stack $100 \mathrm{SiO}_{2}$-coated $\mathrm{Si}$ wafers each possessing a thickness and diameter of $0.3 \mathrm{~mm}$ and $25.4 \mathrm{~mm}$, respectively. The remaining space between the upper most $\mathrm{Si}$ wafer and the cap of the cell is occupied by a flexible aluminum spring-like component, which acts as a spacer preventing the Si wafers from moving around once sealed inside the cell. Figures A.1, A.2, and A.3 are of the schematics to the sample cell main body, cap, and aluminum spacer, respectively, which were all used to seal samples of our model cell membranes. 

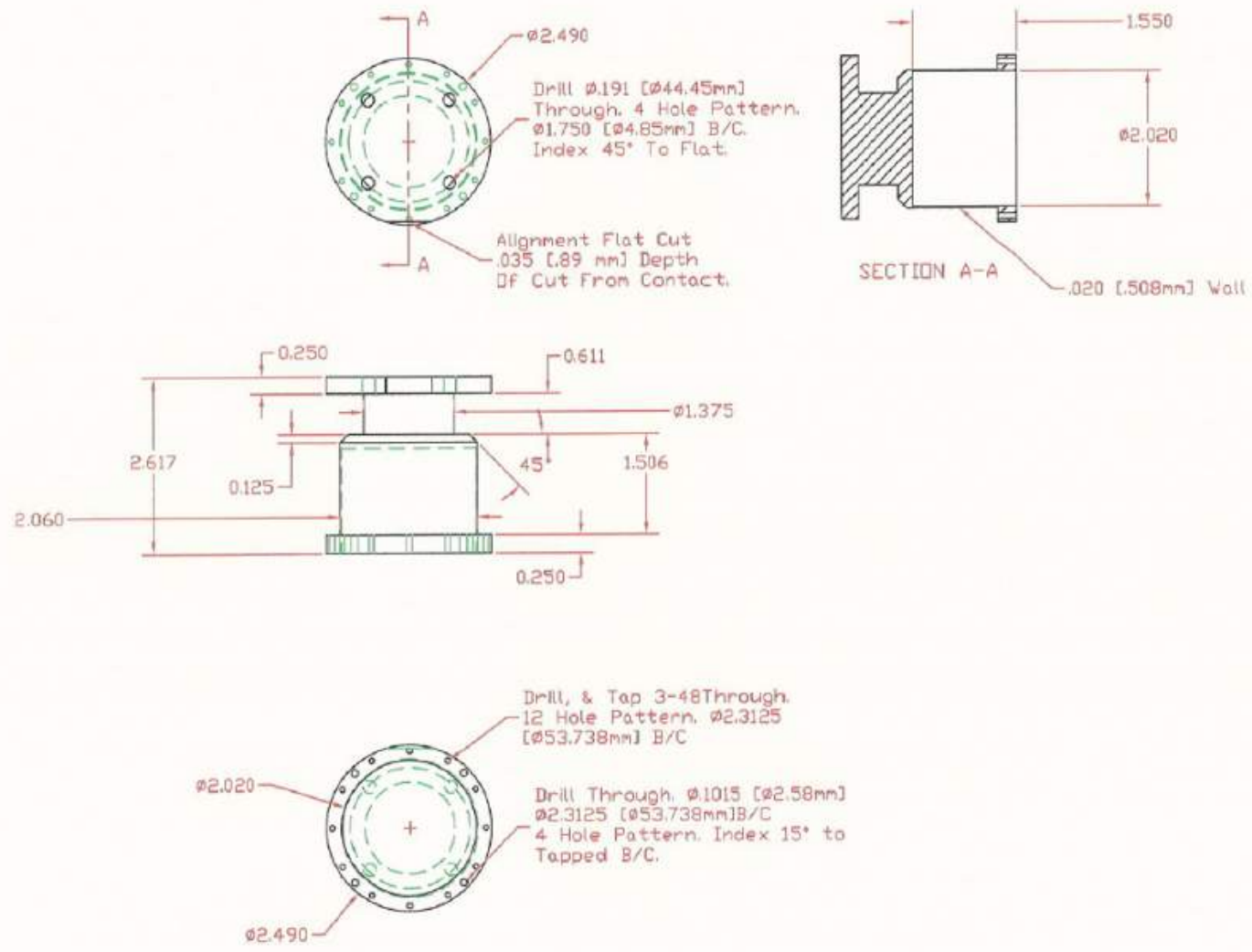

Figure A.1: Schematic of the main body to the sample cell used to contain various model membrane systems for neutron scattering studies. The region illuminated by an incident neutron beam during a scattering measurement is colored in white and shown in section A-A. This illumination window has a thickness of roughly $500 \mu \mathrm{m}$, which helps decrease the probability of unwanted scattering from aluminum. 


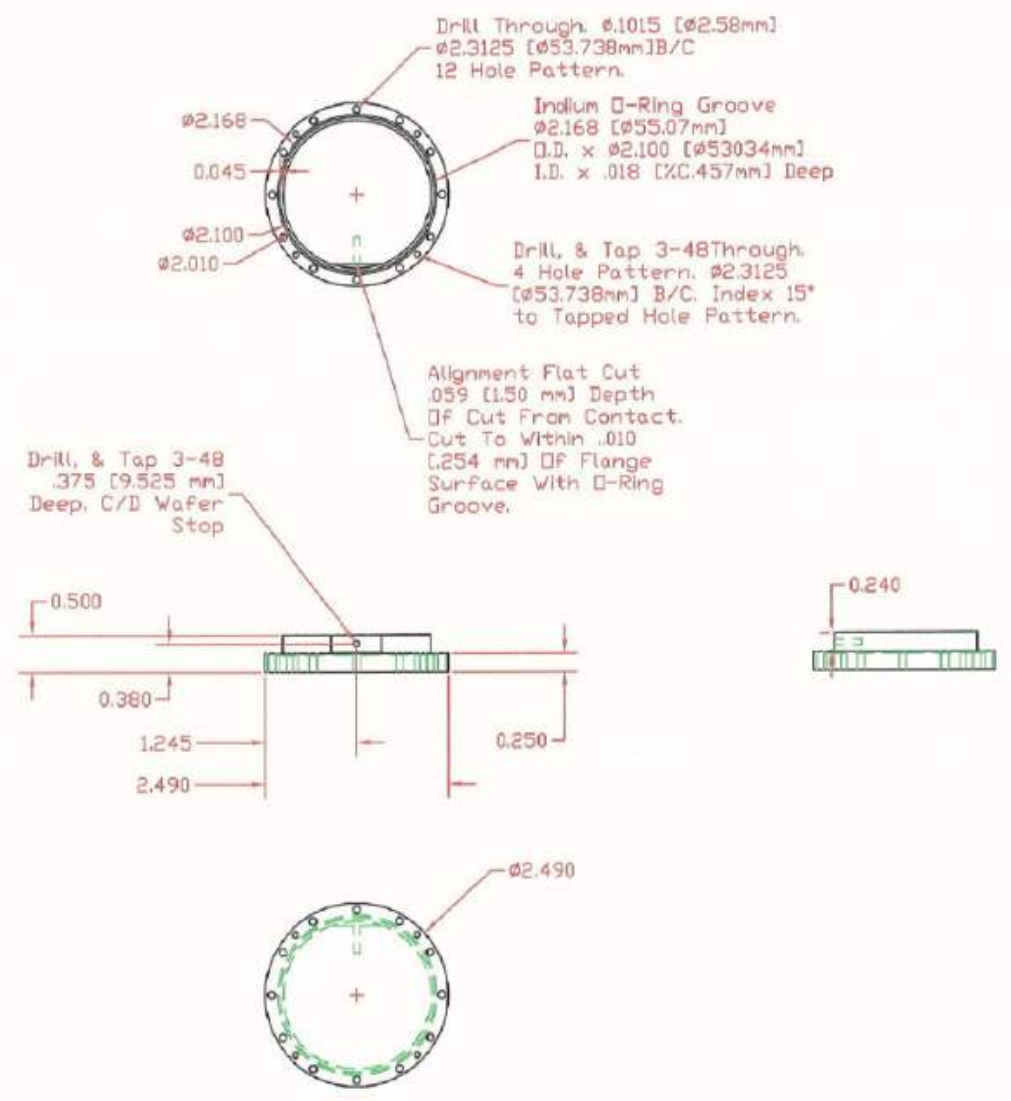

Figure A.2: Schematic of the cap to the sample cell used to contain various model membrane systems for neutron scattering studies. The cap has a small $1.15 \mathrm{~mm}$ wide trench around its flange of for which thin indium wire can be used to seal the inner volume of the cell. 


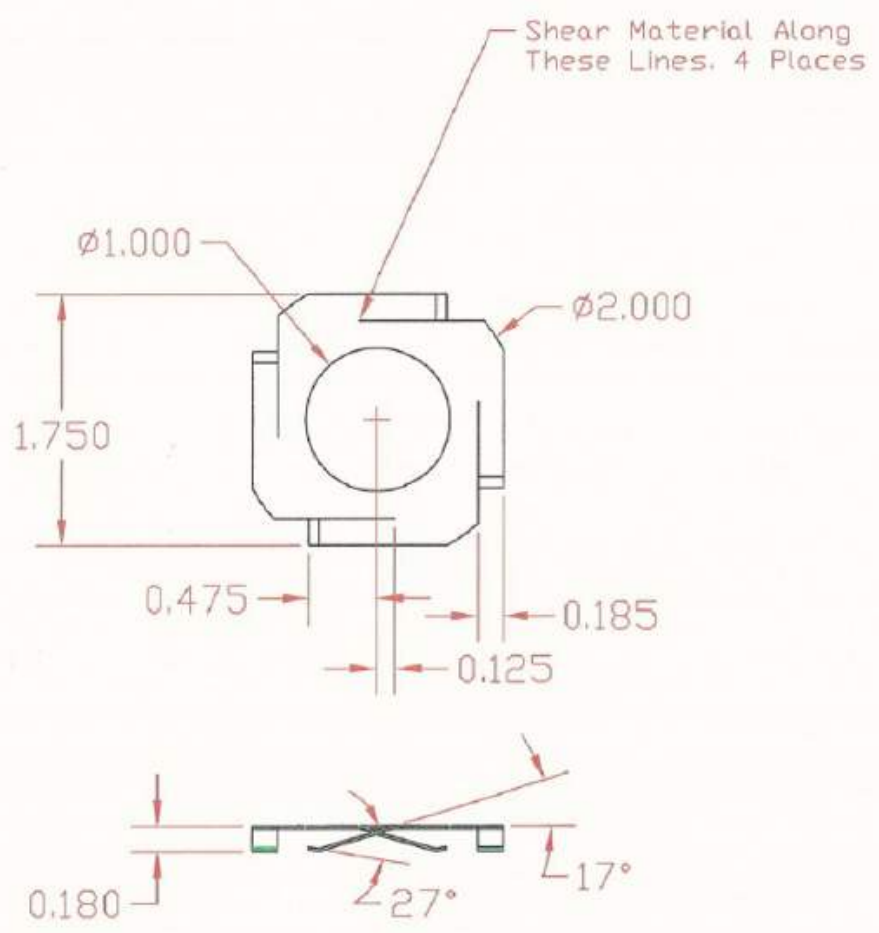

Figure A.3: Schematic of the flexible aluminum spacer to the sample cell used to contain various model membrane systems for neutron scattering studies. The aluminum spacer helps maintain the orientation of Si wafers within the cell while also ensuring they do not shift outside of the scattering volume during data collection. 


\section{Appendix B}

\section{List of Melittin-Treated DMPC}

\section{Samples Measured on HFBS and}

\section{BASIS}

This Appendix contains a comprehensive list of melittin-treated DMPC samples measured on the HFBS and BASIS from September 2013 to September 2016. Sample fabrication parameters, dates of measurement, and figures in which these samples appear are provided. Also included in this appendix are selected elastic scans that have not appeared in the previous text, but offer useful information regarding the effects of melittin on the freezing behavior of water interacting with DMPC membranes treated with various melittin concentrations. All samples were fabricated using procedures described in Chapter 1 and entailed annealing each sample for 3 days at $55^{\circ} \mathrm{C}$ before sealing them with $120 \mu \mathrm{l}$ of $\mathrm{H}_{2} \mathrm{O}$. 
Sample \#1 - $0.1 \mu \mathrm{M}$ melittin-treated DMPC. Measured on the HFBS in December 2014 during which time it was subjected to two thermal cycles with one anneal in between at $55{ }^{\circ} \mathrm{C}$ for approximately 4 hours. Appears in Fig. 4.3 and Fig. 4.5.

Sample \#2 - $0.5 \mu \mathrm{M}$ melittin-treated DMPC. Measured on the HFBS in December 2014 during which time it was subjected to three thermal cycles with two anneals in between at $55{ }^{\circ} \mathrm{C}$. The first and second anneals were for 1.2 hours and 5 hours, respectively. Appears in Fig. 4.3 and Fig. 4.5.

Sample \#3 - $1.0 \mu \mathrm{M}$ melittin-treated DMPC. Measured on the HFBS in December 2014 during which time it was subjected to three thermal cycles with two anneals in between at $55{ }^{\circ} \mathrm{C}$. The first and second anneals were both for 5 hours. Appears in Fig. 4.3 and Fig. 4.5. AFM images for this sample also appear in Fig. 5.10(c) and $5.10(\mathrm{~d})$.

Sample \#4 - $0.5 \mu \mathrm{M}$ melittin-treated DMPC. This sample is identical to sample \#2 and was remeasured on the HFBS in January 2016 after being stored under ambient conditions. A single thermal cycle was performed to compare with the original December 2014 scan (see Fig. 4.3), but results showed the sample quality had degraded and the sample was discarded. Appears in Fig. B.1.

Sample \#5 - $0.5 \mu \mathrm{M}$ melittin-treated DMPC. This sample acted as a 'Backup' to Sample \#4 during annealing experiments on HFBS in January 2016. One thermal cycle was initially performed and showed similar results to previous melittin-treated samples. Therefore, a series of time-dependent anneals were conducted. Appears in Fig. B.1, 4.7, and Table 4.1. 
Sample \#6 - $0.5 \mu \mathrm{M}$ melittin-treated DMPC. Data from a single thermal cycle and one 8 hour anneal was collected from this sample on the HFBS in May 2015. Appears in Fig. B.1.

Sample \#7 - Bare DMPC prepared for QENS measurements on BASIS and measured in May 2016. One single cooling cycle was conducted on BASIS.

Sample \#8 - $0.5 \mu \mathrm{M}$ melittin-treated DMPC. QENS spectra were collected on BASIS in May 2016 for two complete cooling cycles. One anneal was performed at $53{ }^{\circ} \mathrm{C}$ for 2 hours in between cooling cycles. Appears in Fig. 4.10 and Fig. 4.11.

Sample \#9 - $1.0 \mu \mathrm{M}$ melittin-treated DMPC. QENS spectra were collected on BASIS in May 2016 for one cooling cycle.

Sample \#10 - $0.5 \mu \mathrm{M}$ melittin-treated DMPC. QENS spectra were collected on BASIS in Sept 2016 for two cooling cycles and one anneal at $55^{\circ} \mathrm{C}$ for 1 hour. Sample was fabricated at ORNL using Si wafers cleaned via ultraviolet ozone (UVO) method.

Sample \#11 - $1.0 \mu \mathrm{M}$ melittin-treated DMPC. QENS spectra were collected on BASIS in Sept 2016 for two cooling cycles and one anneal at $55{ }^{\circ} \mathrm{C}$ for 5 hours. Sample was fabricated at ORNL using Si wafers cleaned via ultraviolet ozone (UVO) method. 
Table B.1: List of samples measured on HFBS

\begin{tabular}{|cc|c|c|c|}
\hline \multicolumn{2}{|c|}{ Sample Name } & Date of Fabrication & Date of Run & Figures \\
\hline$\# 1$ & $0.1 \mu \mathrm{M}$ melittin & $11 / 10 / 14$ & $12 / 1 / 14 \rightarrow 12 / 3 / 14$ & $4.3,4.5$ \\
\hline$\# 2$ & $0.5 \mu \mathrm{M}$ melittin & $11 / 11 / 14$ & $12 / 3 / 14 \rightarrow 12 / 6 / 14$ & $4.3,4.5$ \\
\hline$\# 3$ & $1.0 \mu \mathrm{M}$ melittin & $11 / 11 / 14$ & $12 / 6 / 14 \rightarrow 12 / 9 / 14$ & $4.3,4.5$ \\
\hline$\# 4$ & $0.5 \mu \mathrm{M}$ melittin & $11 / 11 / 14$ & $01 / 27 / 16$ & B.1 \\
\hline$\# 5$ & $0.5 \mu \mathrm{M}$ melittin & $1 / 9 / 16$ & $1 / 28 / 16 \rightarrow 1 / 31 / 16$ & 4.5 \\
\hline$\# 6$ & $0.5 \mu \mathrm{M}$ melittin & $5 / 10 / 15$ & $5 / 26 / 15 \rightarrow 5 / 27 / 15$ & B.1 \\
\hline
\end{tabular}

Table B.2: List of samples measured on BASIS

\begin{tabular}{|lc|c|c|c|}
\hline \multicolumn{2}{|l|}{ Sample Name } & Date of Fabrication & Date of Run & Figures \\
\hline$\# 7$ & Bare DMPC & $5 / 7 / 16$ & $5 / 19 / 16 \rightarrow 5 / 21 / 16$ & - \\
\hline$\# 8$ & $0.5 \mu \mathrm{M}$ melittin & $5 / 8 / 16$ & $5 / 22 / 16 \rightarrow 5 / 24 / 16$ & $4.10,4.11$ \\
\hline$\# 9$ & $1.0 \mu \mathrm{M}$ melittin & $5 / 9 / 16$ & $5 / 25 / 16 \rightarrow 5 / 26 / 16$ & - \\
\hline$\# 10$ & $0.5 \mu \mathrm{M}$ melittin & $9 / 12 / 16$ & $9 / 16 / 16 \rightarrow 9 / 17 / 16$ & - \\
\hline$\# 11$ & $1.0 \mu \mathrm{M}$ melittin & $8 / 20 / 16$ & $8 / 25 / 16 \rightarrow 9 / 26 / 16$ & - \\
\hline
\end{tabular}




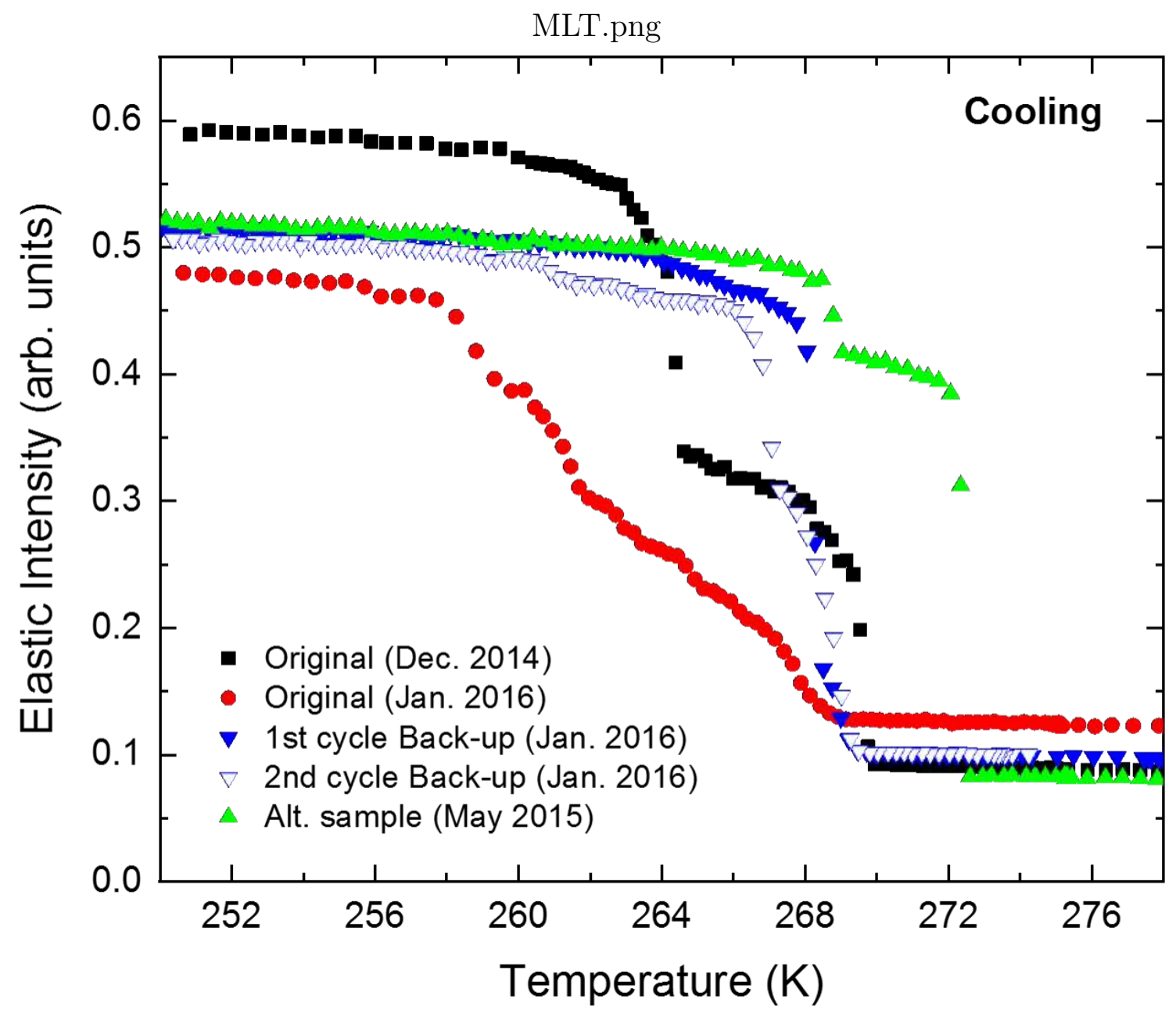

Figure B.1: Raw data of temperature-dependent elastic neutron scans from three different samples of $0.5 \mu \mathrm{M}$ melittin-treated DMPC collected on the HFBS. Black and red data points are from the original $0.5 \mu \mathrm{M}$-sample, which first appears in Fig. 4.3 (black curve). The disagreement between the black and red data suggests the sample quality degraded over the roughly 1 year it was being stored. The open and closed blue triangles are the first and second thermal cycle of a 'backup' sample made for annealing experiments. Finally, the green triangles were measured from an alternative sample used in a brief 2-day experiment in May 2015. 


\section{Appendix $\mathrm{C}$}

\section{Estimating Hydration Level from HFBS Elastic Scans}

This Appendix outlines a simplified method for estimating the amount of hydrogen in a sample comprised of hydrogenous material deposited onto $100 \mathrm{Si}$ wafers which has been measured on the HFBS. A calibration sample was used to determine the number of hydrogen nuclei within a particular scattering volume measured on the HFBS in order to estimate the amount of water hydrating model membranes. The substrates used in the calibration sample were 100 silicon wafers, similar to those used in the deposition of single-supported lipid bilayers $(50 \mathrm{~mm}$ diameter and 0.3 $\mathrm{mm}$ thick). In addition to the $\mathrm{Si}$, the calibration sample consisted of 10 monolayers of $\mathrm{C}_{32} \mathrm{H}_{66}$ molecules vertically oriented and deposited on the surface of each wafer face. By knowing the area occupied by a single alkane molecule $\left(18.84 \AA^{2}\right)$, one is able to estimate the total number of hydrogen nuclei in the sample by evaluating the total change in elastic intensity, assuming all of the sample is within the scattering volume. 


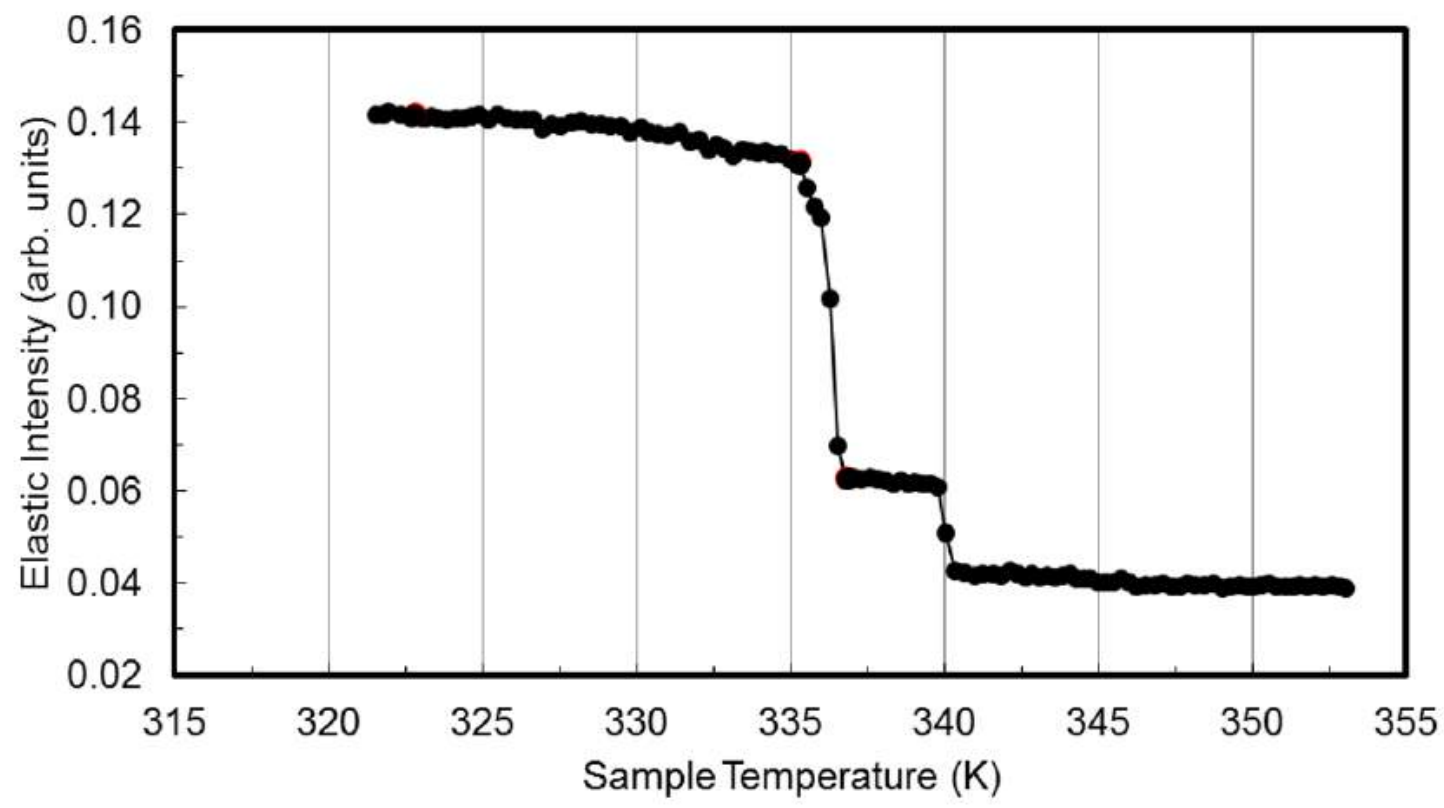

Figure C.1: Elastic neutron scan upon heating of the calibration sample containing 10 monolayers of $\mathrm{C}_{32} \mathrm{H}_{66}$ molecules stacked on the top and bottom of $100 \mathrm{Si}$ wafers measured on the HFBS. 
Figure C.1 shows the elastic scattering intensity summed over all detectors as measured on the HFBS at NIST for a sample of 10 monolayers of $\mathrm{C}_{32}$ alkane material adsorbed onto $100 \mathrm{Si}$ wafers. At high temperatures only the substrate and background contribute to elastic signal whereas at low temperatures all sample material (alkane molecules, substrate, and background) contribute to the elastic intensity. The total elastic scattering intensity measured in all detectors is proportional to the total scattering cross section $\sigma$ of the measured material and can be expressed in the form of a simple summation,

$$
I(T)=\sigma(S i)+\sigma(\text { alkane })+B G
$$

where BG is the background contribution. The incoherent scattering cross sections of nuclei found in the calibration sample: Si, C, and H are 0.004 barns, 0.001 barns, and 80.26 barns, respectively, with hydrogen providing the dominant signal. The difference in intensity of the elastic scan collected from the 10 monolayer $\mathrm{C}_{32} \mathrm{H}_{66}$ sample between 322 and $342 \mathrm{~K}$ is proportional to the alkane cross section.

$$
\sigma(\text { alkane }) \propto I(322 K)-I(342 K)=0.148-0.0395=0.1085
$$

Before finalizing the conversion, the contribution from the high temperature signal must be divided out. Therefore, the ratio of total change in intensity $\Delta \mathrm{I}$ to the intensity at high temperature $I(242 \mathrm{~K})$, as measured on the HFBS, is expressed as,

$$
\frac{\Delta I}{I(242 K)}=\frac{0.1085}{0.0395}=2.747 \text { arb. units }
$$

This ratio represents the immobilization of roughly $1.375 \times 10^{21}$ hydrogen nuclei, 
which has been calculated assuming that the calibration sample is free of any defects and has complete coverage of 10 monolayers of $\mathrm{C}_{32} \mathrm{H}_{66}$ deposited on the top and bottom of 100 Si wafers.

The number of hydrogen nuclei estimated in this way converts to roughly $6.878 \times 10^{20} \mathrm{H}_{2} \mathrm{O}$ molecules. Assuming the bulk density of water, these $\mathrm{H}_{2} \mathrm{O}$ molecules occupy a total volume of $\sim 2.058 \times 10^{19} \mathrm{~nm}^{3}$. Uniformly distributing this water volume over each face of $100 \mathrm{Si}$ wafers (total area $\sim 3.927 \times 10^{17} \mathrm{~nm}^{2}$ ) equates to an equivalent water slab thickness of roughly $52.4 \mathrm{~nm}$. Therefore, one can use the following conversion to estimate the water slab thicknesses in samples of single-supported bilayer membranes, assuming the same number of similar $\mathrm{Si}$ wafers were used as the substrate.

$$
\frac{\Delta I}{I(\text { high } T)}=2.747 \Leftrightarrow 52.4 \mathrm{~nm}
$$

Applying this method to the changes in intensities displayed in the elastic scans of by wet and dry DMPG membranes (Fig. 2.4(a) and Fig. 2.4(d)), we estimate an equivalent water slab thicknesses of approximately $71 \mathrm{~nm}$ and $23 \mathrm{~nm}$, respectively. 


\section{Bibliography}

${ }^{1}$ S. König, E. Sackmann, D. Richter, R. Zorn, C. Carlile, and T. Bayerl, "Molecular dynamics of water in oriented dppc multilayers studied by quasielastic neutron scattering and deuterium-nuclear magnetic resonance relaxation", The Journal of Chemical Physics 100, 3307-3316 (1994).

${ }^{2}$ W. Pfeiffer, T. Henkel, E. Sackmann, W. Knoll, and D. Richter, "Local dynamics of lipid bilayers studied by incoherent quasi-elastic neutron scattering", EPL (Europhysics Letters) 8, 201 (1989).

${ }^{3}$ M. Bai, A. Miskowiec, F. Y. Hansen, H. Taub, T. Jenkins, M. Tyagi, S. O. Diallo, E. Mamontov, K. W. Herwig, and S. K. Wang, "Study of water diffusion on singlesupported bilayer lipid membranes by quasielastic neutron scattering", EPL 98, 48006(1-6) (2012).

${ }^{4}$ A. Miskowiec, Z. Buck, M. C. Brown, H. Kaiser, F. Y. Hansen, G. M. King, H. Taub, R. Jiji, J. W. Cooley, M. Tyagi, S. O. Diallo, E. Mamontov, and K. W. Herwig, "On the freezing behavior and diffusion of water in proximity to single-supported zwitterionic and anionic bilayer membranes", EPL 107, 28008(1-6) (2014).

${ }^{5}$ M.-P. Mingeot-Leclercq, M. Deleu, R. Brasseur, and Y. F. Dufrêne, "Atomic force microscopy of supported lipid bilayers", Nature Protocols 3, 1654-1659 (2008). 
${ }^{6}$ B. Seantier, M.-C. Giocondi, C. Le Grimellec, and P.-E. Milhiet, "Probing supported model and native membranes using afm", Current Opinion in Colloid \& Interface Science 13, 326-337 (2008).

${ }^{7}$ A. Miskowiec, "Studies of water diffusion in the vicinity of single-supported lipid membranes", (May 2014).

${ }^{8}$ D. M. Small, Physical chemistry of lipids (Plenum Press, New York, 1986).

${ }^{9} \mathrm{~J}$. Israelachvili and H. Wennerström, "Role of hydration and water structure in biological and colloidal interactions", Nature 379, 219-225 (1996).

${ }^{10}$ Pure wafer, https://www. purewafer.com/.

${ }^{11}$ K. J. Seu, A. P. Pandey, F. Haque, E. A. Proctor, A. E. Ribbe, and J. S. Hovis, "Effect of surface treatment on diffusion and domain formation in supported lipid bilayers", Biophysical Journal 92, 2445-2450 (2007).

${ }^{12} \mathrm{~K}$. R. Williams and R. S. Muller, "Etch rates for micromachining processing", Journal of Microelectromechanical Systems 5, 256-269 (1996).

${ }^{13}$ D. Stroumpoulis, A. Parra, and M. Tirrell, "A kinetic study of vesicle fusion on silicon dioxide surfaces by ellipsometry", AIChE Journal 52, 2931-2937 (2006).

${ }^{14}$ L. Picas, C. Suárez-Germa, M. Teresa Montero, and J. Hernández-Borrell, "Force spectroscopy study of langmuir- blodgett asymmetric bilayers of phosphatidylethanolamine and phosphatidylglycerol", The Journal of Physical Chemistry B 114, 3543-3549 (2010).

${ }^{15}$ TESt scientific corporation, http://ttscientific.com/.

${ }^{16}$ Sigma-aldrich, https : / / www . sigmaaldrich . com/ catalog / product / sigma / m2272?lang=en\&region=US. 
${ }^{17}$ Genscript, https : //www . genscript . com/peptide/RP10290-Melittin_Honey_ Bee.html.

${ }^{18}$ K. Sugihara, M. Delai, I. Szendro, O. Guillaume-Gentil, J. Vörös, and T. Zambelli, "Simultaneous owls and eis monitoring of supported lipid bilayers with the pore forming peptide melittin", Sensors and Actuators B: Chemical 161, 600-606 (2012).

${ }^{19}$ G. Klocek, T. Schulthess, Y. Shai, and J. Seelig, "Thermodynamics of melittin binding to lipid bilayers. aggregation and pore formation", Biochemistry 48, 25862596 (2009).

${ }^{20} \mathrm{H}$. Vogel, "Incorporation of melittin into phosphatidylcholine bilayers: study of binding and conformational changes", FEBS Letters 134, 37-42 (1981).

${ }^{21}$ N. Lu, K. Yang, B. Yuan, and Y. Ma, "Molecular response and cooperative behavior during the interactions of melittin with a membrane: dissipative quartz crystal microbalance experiments and simulations", The Journal of Physical Chemistry B 116, 9432-9438 (2012).

${ }^{22}$ A. Meyer, R. M. Dimeo, P. M. Gehring, and D. A. Neumann, "The high-flux backscattering spectrometer at the nist center for neutron research", Rev. Sci. Instrum 74, 2759-2777 (2003).

${ }^{23}$ N. Kucerka, M. A. Kiselev, and P. Balgavỳ, "Determination of bilayer thickness and lipid surface area in unilamellar dimyristoylphosphatidylcholine vesicles from smallangle neutron scattering curves: a comparison of evaluation methods", European Biophysics Journal 33, 328-334 (2004). 
${ }^{24}$ A. Rønnest, G. Peters, F. Y. Hansen, H. Taub, and A. Miskowiec, "Structure and dynamics of water and lipid molecules in charged anionic dmpg lipid bilayer membranes", The Journal of chemical physics 144, 144904(1-15) (2016).

${ }^{25}$ E. Mamontov and K. W. Herwig, "A time-of-flight backscattering spectrometer at the spallation neutron source, basis", Review of Scientific Instruments 82, 085109(110) (2011).

${ }^{26}$ R. T. Azuah, L. R. Kneller, Y. Qiu, P. L. Tregenna-Piggott, C. M. Brown, J. R. Copley, and R. M. Dimeo, "Dave: a comprehensive software suite for the reduction, visualization, and analysis of low energy neutron spectroscopic data", Journal of Research of the National Institute of Standards and Technology 114, 341-358 (2009).

${ }^{27}$ R. J. Chudley, C. T. Elliott, "Neutron scattering from a liquid on a jump diffusion model", Proc. of Physical Society 77(2), 353-361 (1961).

${ }^{28}$ M. Bee, Quasielastic neutron scattering (IOP Publishing Ltd, Bristol, England, 1988).

${ }^{29}$ P. T. Hall and D. K. Ross, "Incoherent neutron scattering functions for random jump diffusion in bounded infinite media", Molecular Physics 42(3), 673-682 (1981).

${ }^{30}$ J. Teixeira, M.-C. Bellissent-Funel, S.-H. Chen, and A.-J. Dianoux, "Experimental determination of the nature of diffusive motions of water molecules at low temperatures", Physical Review A 31, 1913-1917 (1985).

${ }^{31}$ W. S. Price, H. Ide, and Y. Arata, "Self-diffusion of supercooled water to $238 \mathrm{k}$ using pgse nmr diffusion measurements", The Journal of Physical Chemistry A 103, 448450 (1999). 
${ }^{32}$ C. Faure, L. Bonakdar, and E. J. Dufourc, "Determination of dmpc hydration in the $\mathrm{l} \alpha$ and $\mathrm{l} \beta$ phases by $2 \mathrm{~h}$ solid state nmr of d2o", FEBS Letters 405, 263-266 (1997).

${ }^{33}$ F. Y. Hansen, G. Peters, H. Taub, and A. Miskowiec, "Diffusion of water and selected atoms in dmpc lipid bilayer membranes", The Journal of Chemical Physics 137, 204910(1-15) (2012).

${ }^{34}$ J. Das, E. Flenner, and I. Kosztin, "Anomalous diffusion of water molecules in hydrated lipid bilayers", The Journal of Chemical Physics 139, 065102(1-9) (2013).

${ }^{35}$ Y. von Hansen, S. Gekle, and R. R. Netz, "Anomalous anisotropic diffusion dynamics of hydration water at lipid membranes", Physical Review Letters 111, 118103(15) (2013).

${ }^{36}$ J. Yang, C. Calero, and J. Marti, "Diffusion and spectroscopy of water and lipids in fully hydrated dimyristoylphosphatidylcholine bilayer membranes", The Journal of Chemical Physics 140, 104901(1-13) (2014).

${ }^{37}$ F. S. Varley, "Neutron scattering lengths and cross sections", Neutron News 3, 2937 (1992).

${ }^{38}$ Lakeshore cryogenics, https : / / www . lakeshore . com / products / Cryogenic Temperature-Controllers/Model-336/Pages/Overview . aspx.

${ }^{39}$ K. Röttger, A. Endriss, J. Ihringer, S. Doyle, and W. Kuhs, "Lattice constants and thermal expansion of h2o and d2o ice ih between 10 and 265 k", Acta Crystallographica Section B: Structural Science 50, 644-648 (1994). 
${ }^{40}$ R. J. Dubos, "Studies on a bactericidal agent extracted from a soil bacillus: i. preparation of the agent. its activity in vitro", The Journal of Experimental Medicine 70, $1-10(1939)$.

${ }^{41}$ A. A. Bahar and D. Ren, "Antimicrobial peptides", Pharmaceuticals 6, 1543-1575 (2013).

${ }^{42}$ X. Zhao, H. Wu, H. Lu, G. Li, and Q. Huang, "Lamp: a database linking antimicrobial peptides", PLoS One 8, e66557(1-6) (2013).

${ }^{43}$ A. Miskowiec, Z. Buck, F. Y. Hansen, H. Kaiser, H. Taub, M. Tyagi, S. Diallo, E. Mamontov, and K. Herwig, "On the structure and dynamics of water associated with single-supported zwitterionic and anionic membranes", The Journal of Chemical Physics 146, 125102(1-15) (2017).

${ }^{44}$ W. C. Wimley, "Describing the mechanism of antimicrobial peptide action with the interfacial activity model", ACS Chemical Biology 5, 905-917 (2010).

${ }^{45}$ M.-T. Lee, T.-L. Sun, W.-C. Hung, and H. W. Huang, "Process of inducing pores in membranes by melittin", Proceedings of the National Academy of Sciences, U.S.A. 110, 14243-14248 (2013).

${ }^{46}$ D. Allende, S. Simon, and T. J. McIntosh, "Melittin-induced bilayer leakage depends on lipid material properties: evidence for toroidal pores", Biophysical Journal 88, $1828-1837$ (2005).

${ }^{47}$ A. Naito, T. Nagao, K. Norisada, T. Mizuno, S. Tuzi, and H. Saitô, "Conformation and dynamics of melittin bound to magnetically oriented lipid bilayers by solid-state 31 p and 13 c nmr spectroscopy", Biophysical Journal 78, 2405-2417 (2000). 
${ }^{48}$ K. A. Brogden, "Antimicrobial peptides: pore formers or metabolic inhibitors in bacteria?", Nature Reviews Microbiology 3, 238-250 (2005).

${ }^{49}$ D. J. Son, J. W. Lee, Y. H. Lee, H. S. Song, C. K. Lee, and J. T. Hong, "Therapeutic application of anti-arthritis, pain-releasing, and anti-cancer effects of bee venom and its constituent compounds", Pharmacology \& therapeutics 115, 246-270 (2007).

${ }^{50}$ G. Gajski and V. Garaj-Vrhovac, "Melittin: a lytic peptide with anticancer properties", Environmental Toxicology and Pharmacology 36, 697-705 (2013).

${ }^{51}$ M. Wachinger, A. Kleinschmidt, D. Winder, N. von Pechmann, A. Ludvigsen, M. Neumann, R. Holle, B. Salmons, V. Erfle, and R. Brack-Werner, "Antimicrobial peptides melittin and cecropin inhibit replication of human immunodeficiency virus 1 by suppressing viral gene expression.", Journal of General Virology 79, 731-740 (1998).

${ }^{52}$ R. Bazzo, M. J. Tappin, A. Pastore, T. S. Harvey, J. A. Carver, and I. D. Campbell, "The structure of melittin", The FEBS Journal 173, 139-146 (1988).

${ }^{53}$ T. C. Terwilliger, L. Weissman, and D. Eisenberg, "The structure of melittin in the form i crystals and its implication for melittin's lytic and surface activities", Biophysical Journal 37, 353-361 (1982).

${ }^{54}$ S. McNicholas, E. Potterton, K. Wilson, and M. Noble, "Presenting your structures: the ccp4mg molecular-graphics software", Acta Crystallographica Section D: Biological Crystallography 67, 386-394 (2011).

${ }^{55}$ D. S. Perekalin, V. V. Novikov, A. A. Pavlov, I. A. Ivanov, N. Y. Anisimova, A. N. Kopylov, D. S. Volkov, I. F. Seregina, M. A. Bolshov, and A. R. Kudinov, "Selective 
ruthenium labeling of the tryptophan residue in the bee venom peptide melittin", Chemistry-A European Journal 21, 4923-4925 (2015).

${ }^{56}$ A. S. Ladokhin and S. H. White, "Folding of amphipathic $\alpha$-helices on membranes: energetics of helix formation by melittin", Journal of Molecular Biology 285, 13631369 (1999).

${ }^{57}$ P. F. Almeida, A. S. Ladokhin, and S. H. White, "Hydrogen-bond energetics drive helix formation in membrane interfaces", Biochimica et Biophysica Acta (BBA)Biomembranes 1818, 178-182 (2012).

${ }^{58}$ L. Yang, T. A. Harroun, T. M. Weiss, L. Ding, and H. W. Huang, "Barrel-stave model or toroidal model? a case study on melittin pores", Biophysical Journal 81, 1475-1485 (2001).

${ }^{59}$ A. I. Kolesnikov, J.-M. Zanotti, C.-K. Loong, P. Thiyagarajan, A. P. Moravsky, R. O. Loutfy, and C. J. Burnham, "Anomalously soft dynamics of water in a nanotube: a revelation of nanoscale confinement", Physical Review Letters 93, 035503(1-4) (2004).

${ }^{60} \mathrm{H}$. Raghuraman and A. Chattopadhyay, "Melittin: a membrane-active peptide with diverse functions", Bioscience Reports 27, 189-223 (2007).

${ }^{61}$ A. M. Baró and R. G. Reifenberger, Atomic force microscopy in liquid: biological applications (John Wiley \& Sons, 2012).

${ }^{62}$ T. R. Matin, K. P. Sigdel, M. Utjesanovic, B. P. Marsh, F. Gallazzi, V. F. Smith, I. Kosztin, and G. M. King, "Single-molecule peptide-lipid affinity assay reveals interplay between solution structure and partitioning", Langmuir 33, 4057-4065 (2017). 
${ }^{63}$ P. S. Cremer and S. G. Boxer, "Formation and spreading of lipid bilayers on planar glass supports", The Journal of Physical Chemistry B 103, 2554-2559 (1999).

${ }^{64}$ T. D. Bradrick, A. Philippetis, and S. Georghiou, "Stopped-flow fluorometric study of the interaction of melittin with phospholipid bilayers: importance of the physical state of the bilayer and the acyl chain length", Biophysical Journal 69, 1999-2010 (1995).

${ }^{65}$ F.-Y. Chen, M.-T. Lee, and H. W. Huang, "Evidence for membrane thinning effect as the mechanism for peptide-induced pore formation", Biophysical Journal 84, $3751-3758$ (2003).

${ }^{66}$ H. W. Huang, F.-Y. Chen, and M.-T. Lee, "Molecular mechanism of peptide-induced pores in membranes", Physical Review Letters 92, 198304(1-4) (2004).

${ }^{67}$ S. Ludtke, K. He, and H. Huang, "Membrane thinning caused by magainin 2", Biochemistry 34, 16764-16769 (1995).

${ }^{68}$ A. Mecke, D.-K. Lee, A. Ramamoorthy, B. G. Orr, and M. M. B. Holl, "Membrane thinning due to antimicrobial peptide binding: an atomic force microscopy study of msi-78 in lipid bilayers", Biophysical Journal 89, 4043-4050 (2005).

${ }^{69}$ P. D. Rakowska, H. Jiang, S. Ray, A. Pyne, B. Lamarre, M. Carr, P. J. Judge, J. Ravi, U. I. Gerling, B. Koksch, et al., "Nanoscale imaging reveals laterally expanding antimicrobial pores in lipid bilayers", Proceedings of the National Academy of Sciences, U.S.A. 110, 8918-8923 (2013).

${ }^{70}$ S. J. Johnson, T. M. Bayerl, D. C. McDermott, G. W. Adam, A. R. Rennie, R. K. Thomas, and E. Sackmann, "Structure of an adsorbed dimyristoylphosphatidyl- 
choline bilayer measured with specular reflection of neutrons", Biophysical Journal 59, 289-294 (1991).

${ }^{71}$ C. A. Bippes and D. J. Muller, "High-resolution atomic force microscopy and spectroscopy of native membrane proteins", Reports on Progress in Physics 74, 086601(1-43) (2011).

${ }^{72}$ C. Lee and C. D. Bain, "Raman spectra of planar supported lipid bilayers", Biochimica et Biophysica Acta (BBA)-Biomembranes 1711, 59-71 (2005). 


\section{VITA}

Zachary Buck was born in Thornton, Colorado. Although he relocated several times growing up, he spent most of his life in southern NJ. In May 2012, Zack graduated from Rowan University, NJ with a Bachelor of Science degree in Physics. He began his graduate work at the University of Missouri - Columbia in August, 2012 under the supervision of Dr. Haskell Taub. Between May and November 2016, Zack participated in a Department of Energy funded Office of Science Graduate Student Research program at Oak Ridge National Laboratory, TN where he conducted a significant part of his thesis research. In May, 2018, he graduated from the University of Missouri - Columbia with a Ph.D. in Physics and is pursuing a career in neutron scattering and alternative energy material science research. 